\title{
Ketene-Ketenimine Rearrangements in the Gas Phase and in Polar Media. 1,3-Migration Intermediates and Sequential Transition States
}

Justin J. Finnerty and Curt Wentrup*

Chemistry Building, School of Molecular and Microbial Sciences, The University of Queensland, Brisbane, Qld 4072, Australia.wentrup@uq.edu.au

\section{Content:}

Table S1. Calculated data for $\alpha$-oxoketene $\mathbf{4}$ - $\alpha$-oxoketenimines 4 interconversion page $\mathrm{S} 1$ Table S2. Calculated data for $\alpha$-oxoketene $\mathbf{1}$ - $\alpha$-oxoketenimines $\mathbf{3}$ interconversion in the gas phase page S2 Table S3. Calculated data for $\alpha$-oxoketene $\mathbf{1}$ - $\alpha$-oxoketenimines 3 interconversion in polar medium page S3 Energy profile for ketenes, ketenimines and 1,3-migration transition structures for the gas phase: Transition states for 1,3-migration of all substituents and for rotation of amino groups Calculated data for $\alpha$-imidoylketenes 1 - $\alpha$-oxoketenimines 3 interconversion: page page 55 page $\mathrm{S} 6$ page $\mathrm{S} 21$

Table S1. Calculated (B3LYP/6-311+G(3df,2p)//B3LYP/6-31G*) energy data for the 1,3-migration in -oxoketenes 4 compared to the G2(MP2,SVP) values (Difference column) Data from references 6a and 7. Calculated energy values are for 298.15K.

\begin{tabular}{|c|c|c|c|c|c|}
\hline & \multicolumn{3}{|c|}{ B3LYP } & \multicolumn{2}{|c|}{ G2(MP2, SVP) } \\
\hline & $\Delta \mathrm{H}$ & $\Delta S$ & $\Delta \mathrm{G}$ & $\Delta$ & Difference \\
\hline $\begin{array}{l}\text { Compound/ } \\
\text { Substituent }\end{array}$ & $/ \mathrm{kJ} \mathrm{mol}^{-1}$ & $/ \mathrm{JK}^{-1} \mathrm{~mol}^{-1}$ & $/ \mathrm{kJ} \mathrm{mol}^{-1}$ & $/ \mathrm{kJ} \mathrm{mol}^{-1}$ & $/ \mathrm{kJ} \mathrm{mol}^{-1}$ \\
\hline $4 \mathrm{Br}$ & 25.5 & -18.1 & 30.9 & 37 & 8.0 \\
\hline $4 \mathrm{CH}_{3}$ & 198.2 & -11.6 & 201.7 & 206 & 4.3 \\
\hline $4 \mathrm{Cl}$ & 37.6 & -16.3 & 42.5 & 53 & 10.2 \\
\hline $4 \mathrm{~F}$ & 78.7 & -17.2 & 83.9 & 89 & 5.0 \\
\hline $4 \mathrm{H}$ & 137.3 & -10.1 & 140.3 & 143 & 2.4 \\
\hline
\end{tabular}




$\begin{array}{lrrrrr}\mathbf{4} \mathrm{NH}_{2} & 69.5 & -11.8 & 73.1 & 70 & -3.5 \\ \mathbf{4 N}\left(\mathrm{CH}_{3}\right)_{2}{ }^{\mathrm{a}} & 30.9 & -20.6 & 37.0 & & -3.1 \\ \mathbf{4 N}\left(\mathrm{CH}_{3}\right)_{2}{ }^{\mathrm{b}} & 36.3 & -23.7 & 43.3 & 34 & -9.5 \\ \mathbf{4} \mathrm{OH} & 107.1 & -14.2 & 111.4 & 114 & 3.1 \\ \mathbf{4} \mathrm{Ph}^{\mathrm{c}} & & & 150.7 & & \\ \mathbf{4 S H} & 39.9 & -20.4 & 46.0 & 51 & 5.5\end{array}$

In the $\mathrm{N}\left(\mathrm{CH}_{3}\right)_{2}$ case the symmetric 1,3 migration structure was an intermediate. The migration therefore had two unsymmetric degenerate transition states either side of the intermediate. The results for both the intermediate $\left({ }^{a}\right)$ and the transition state $\left(^{b}\right)$ are reported. ( ${ }^{\mathrm{c}}$ ) George, L.; Wentrup, C. Org. Biomol. Chem. 2005, 3, 2998-3000.

$\overline{\text { Table S2. Relative free energy }\left(\Delta G ; \mathrm{kJ} \mathrm{mol}^{-1} \text {, in descending order of transition }\right.}$ state energy) for 1,3-migration of substituents from imidoylketene $\mathbf{1}$ via fourmembered ring transition state TS2 to oxoketenimine 3 . The $E$ and $Z$ configurations of the imine group in the imidoylketenes are denoteed $i E$ and $i Z$. (See footnote 10)

\begin{tabular}{rrrrrr} 
Substituent & 1 sZ & 1 sE & TS2 & $3 s E$ & $3 s Z$ \\
\hline $\mathrm{CH}_{3} i E$ & 5.68 & 0.00 & 257.32 & 3.69 & 7.74 \\
$\mathrm{CH}_{3} i Z$ & 0.66 & 0.00 & 237.48 & 4.75 & 8.80 \\
$\mathrm{Ph} i E$ & 2.56 & 0.00 & 201.70 & 5.01 & 0.87 \\
$\mathrm{Ph} i Z$ & -0.86 & 0.00 & 185.27 & 7.10 & 2.96 \\
$\mathrm{H} i E$ & 7.10 & 0.00 & 199.46 & 9.47 & 16.20 \\
$\mathrm{H} i Z$ & 2.34 & 0.00 & 182.47 & 13.29 & 20.01 \\
$\mathrm{OH} i E$ & 7.44 & 0.00 & 143.57 & -14.25 & -11.44 \\
$\mathrm{OH}_{i Z}$ & 2.96 & 0.00 & 120.20 & -26.08 & -23.27 \\
$\mathrm{OCH}_{3} i E$ & 7.34 & 0.00 & 125.27 & -16.78 & -16.39 \\
$\mathrm{OCH}_{3} i Z$ & 13.68 & 0.00 & 109.44 & -26.06 & -25.67 \\
$\mathrm{NH}_{2} i E$ & 4.94 & 0.00 & 113.19 & -18.88 & -18.40 \\
$\mathrm{NH}_{2} i Z$ & -2.15 & 0.00 & 97.50 & -23.11 & -22.64
\end{tabular}




$\begin{array}{rrrrrr}\mathrm{F} i E & 6.97 & 0.00 & 110.50 & -21.68 & -18.19 \\ \mathrm{~F} i Z & 1.76 & 0.00 & 96.46 & -13.75 & -10.26 \\ \mathrm{~N}\left(\mathrm{CH}_{3}\right)_{2} i E & 8.48 & 0.00 & 78.74 & -26.73 & -17.10 \\ \mathrm{~N}\left(\mathrm{CH}_{3}\right)_{2} i Z & 2.14 & 0.00 & 70.07 & -27.02 & -17.39 \\ \mathrm{SH} i E & 3.32 & 0.00 & 89.91 & -9.09 & -8.97 \\ \mathrm{SH} i Z & -2.61 & 0.00 & 67.65 & -11.32 & -11.20 \\ \mathrm{Cl} i E & 7.24 & 0.00 & 70.36 & -18.68 & -15.29 \\ \mathrm{Cl} i Z & 1.98 & 0.00 & 61.18 & -4.88 & -1.49 \\ \mathrm{SCH}_{3} i E & -24.40 & 0.00 & 62.75 & -38.55 & -37.99 \\ \mathrm{SCH} \mathrm{H}_{3} i Z & -6.81 & 0.00 & 55.17 & -27.81 & -27.25 \\ \mathrm{Br} i E & 6.84 & 0.00 & 55.92 & -21.86 & -20.81 \\ \mathrm{Br} i Z & 1.94 & 0.00 & 48.30 & -5.19 & -4.13\end{array}$

Table S3. Relative free energy $\left(\Delta G ; \mathrm{kJ} \mathrm{mol}^{-1}\right)$ for 1,3 migration of substituents in polar medium (acetonitrile; $\varepsilon=36.64$ ) from imidoyl-ketene 1 via four-membered transition state TS2 to oxo-ketenimine 3. The $E$ and $Z$ configurations of the imine groups in the imidoyl ketenes are denoted $i E$ and $i Z$. (See footnote 10. Same order of substituents as in Table S2)

\begin{tabular}{lcccrr} 
Substituent & $\mathbf{1} s Z$ & $\mathbf{1} s E$ & TS2 & 3 sE & 3 sZ \\
\hline $\mathrm{CH}_{3} i E$ & 6.53 & 0.00 & 253.38 & -13.28 & -11.29 \\
$\mathrm{CH}_{3} i Z$ & 0.47 & 0.00 & 246.65 & -14.08 & -12.09 \\
$\mathrm{Ph} i E$ & 4.84 & 0.00 & 180.11 & -11.39 & -11.91 \\
$\mathrm{Ph} i Z$ & -0.13 & 0.00 & 167.96 & -11.83 & -12.36 \\
$\mathrm{H} i E$ & 10.28 & 0.00 & 212.68 & -4.30 & 1.57 \\
$\mathrm{H} i Z$ & 4.46 & 0.00 & 194.75 & -3.30 & 2.56 \\
$\mathrm{OH} i E$ & 8.54 & 0.00 & 126.61 & -34.18 & -30.41 \\
$\mathrm{OH} i Z$ & 7.54 & 0.00 & 114.72 & -38.34 & -34.57
\end{tabular}




$\begin{array}{lccccc}\mathrm{OCH}_{3} i E & 7.99 & 0.00 & 114.64 & -31.98 & -32.96 \\ \mathrm{OCH}_{3} i Z & 8.07 & 0.00 & 100.07 & -42.50 & -43.47 \\ \mathrm{NH}_{2} i E & 2.15 & 0.00 & 94.98 & -38.47 & -43.79 \\ \mathrm{NH}_{2} i Z & -5.40 & 0.00 & 78.67 & -40.65 & -45.96 \\ \mathrm{~F} i E & 7.57 & 0.00 & 99.90 & -32.04 & -30.52 \\ \mathrm{~F} i Z & 7.49 & 0.00 & 86.33 & -32.12 & -30.60 \\ \mathrm{~N}\left(\mathrm{CH}_{3}\right)_{2} i E & -0.23 & 0.00 & 64.24 & -33.81 & -44.22 \\ \mathrm{~N}\left(\mathrm{CH}_{3}\right)_{2} i Z & -7.38 & 0.00 & 45.19 & -41.84 & -52.25 \\ \mathrm{SH} i E & 0.77 & 0.00 & 73.83 & -27.60 & -30.19 \\ \mathrm{SH} i Z & -26.89 & 0.00 & 54.54 & -28.89 & -31.49 \\ \mathrm{Cl} i E & 8.13 & 0.00 & 42.87 & -30.06 & -29.29 \\ \mathrm{Cl} \mathrm{iZ} & 2.31 & 0.00 & 30.97 & -23.85 & -23.08 \\ \mathrm{SCH} & -15.64 & 0.00 & 55.62 & -46.90 & -47.90 \\ \mathrm{SCH} & -8.60 & 0.00 & 42.58 & -42.92 & -43.92 \\ \mathrm{Br} i E & 7.59 & 0.00 & 30.01 & -33.45 & -33.59 \\ \mathrm{Br} \mathrm{iZ} & 2.37 & 0.00 & 24.00 & -24.23 & -24.38\end{array}$




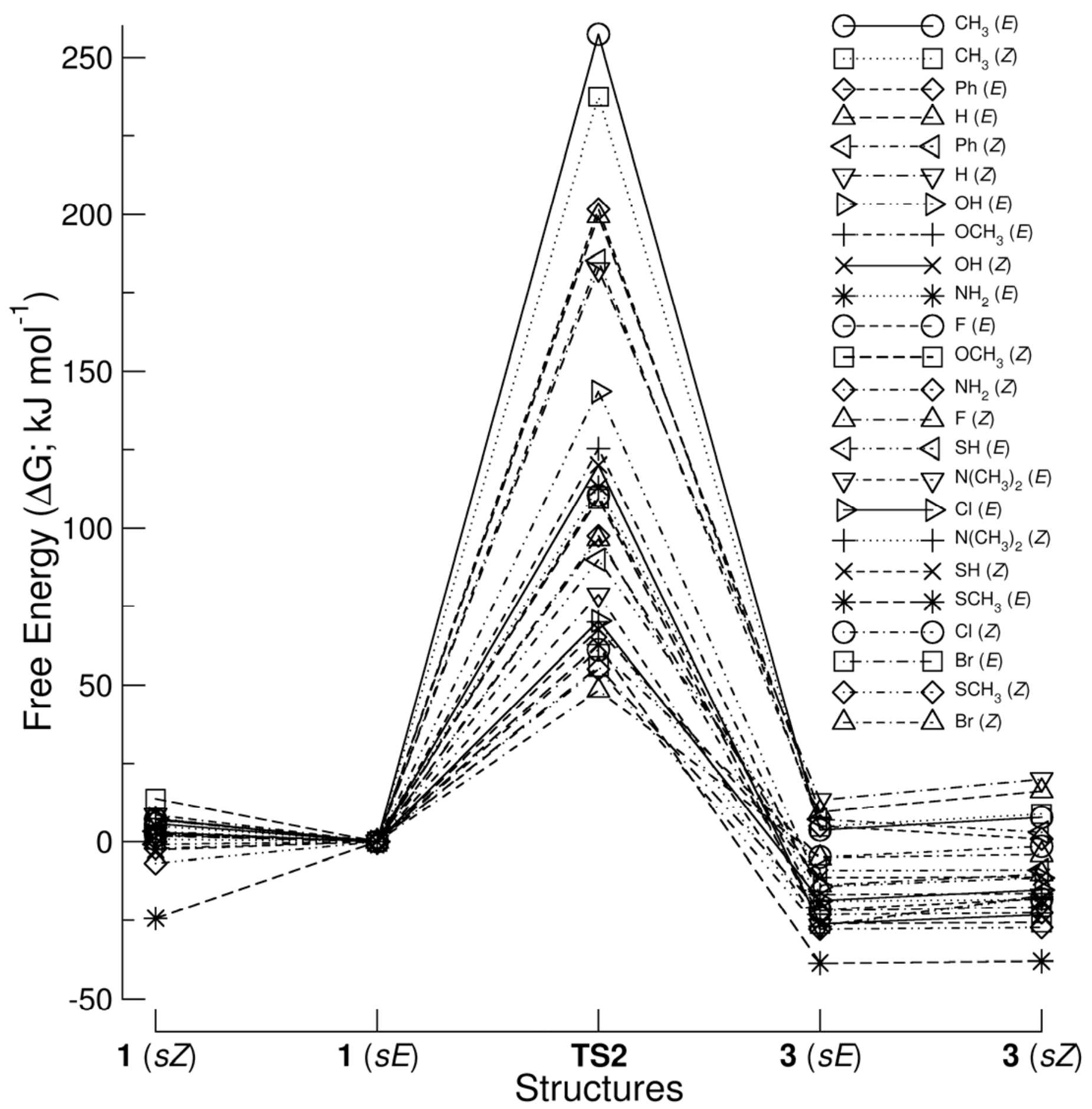

Figure S1. Potential energy paths for the 1,3-migrations of groups in the reaction $\mathbf{1} \rightarrow \mathbf{3}$ (calculations for the gas phase, $\varepsilon=0)$.

Figures S2-S16: Transition states for 1,3-migration of all substituents and for rotation of amino groups: 
A

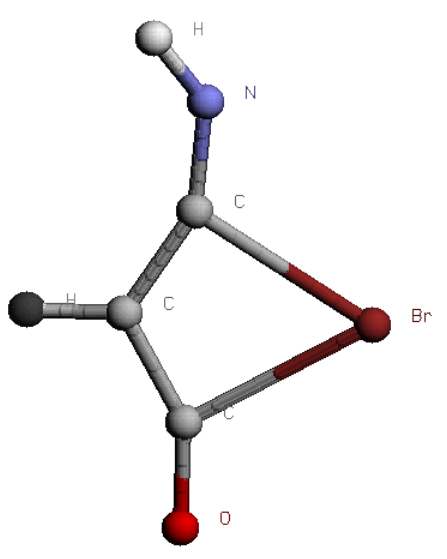

B

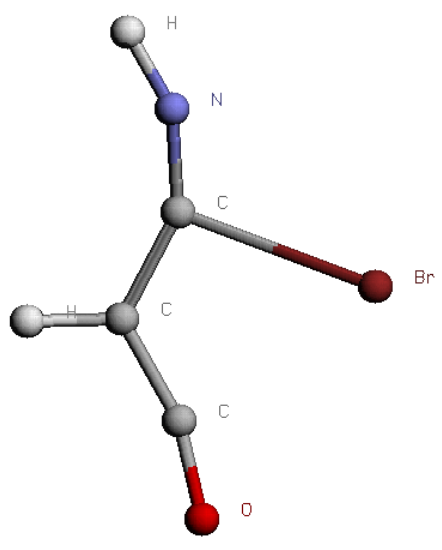

C

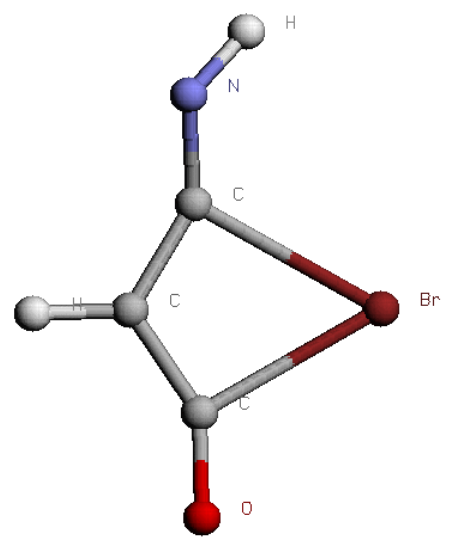

D

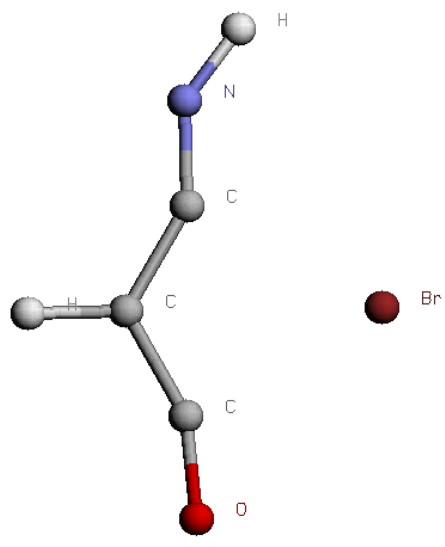

Figure S2 Calculated Br migration transition states for ketene $\mathbf{1} /$ ketenimine 3 (A and C for the gas phase; B and $\mathrm{D}$ in simulated acetonitrile solvent) 


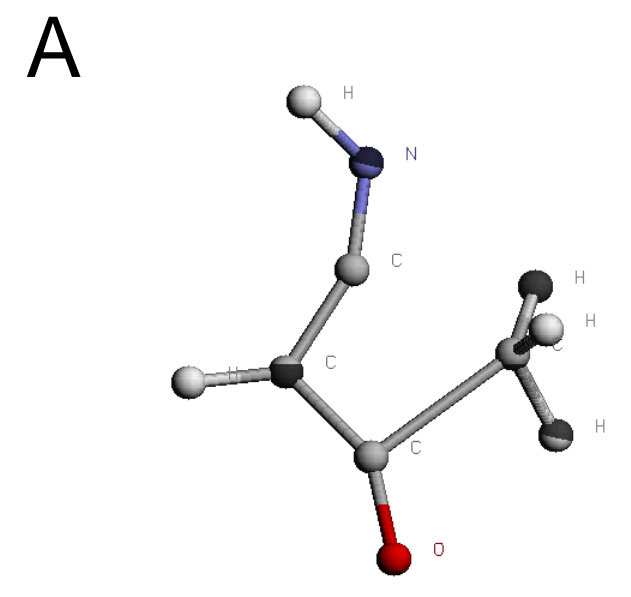

B

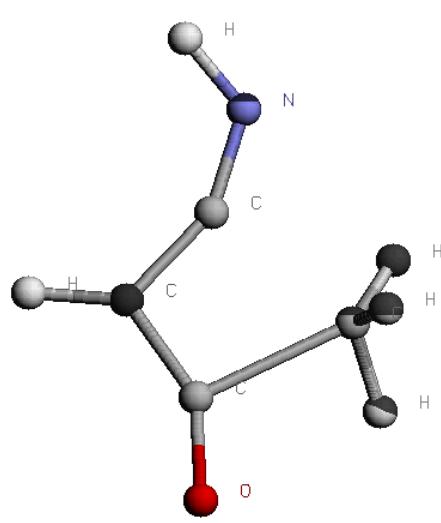

C

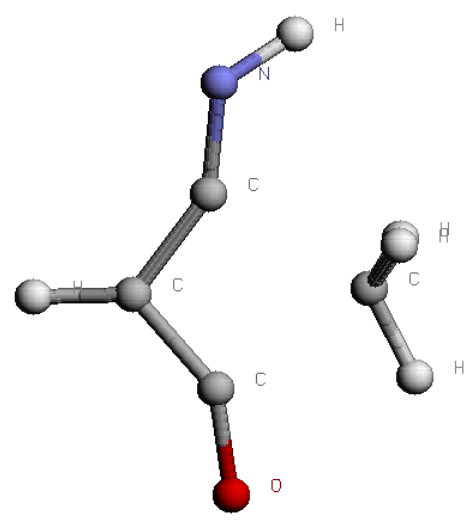

$\mathrm{D}$

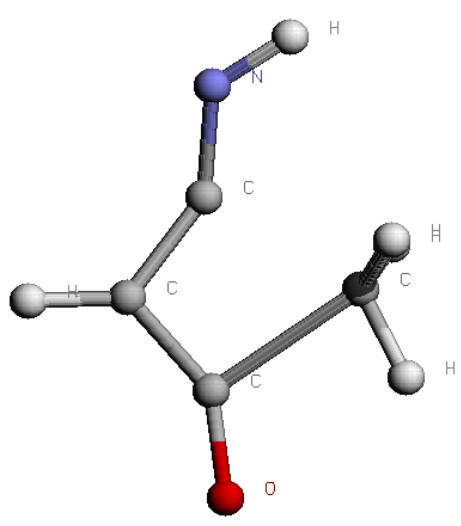

Figure S3 Calculated methyl migration transition states (for ketene $\mathbf{1} /$ ketenimine 3 (A and C for the gas phase; $\mathrm{B}$ and $\mathrm{D}$ in simulated acetonitrile solvent) 


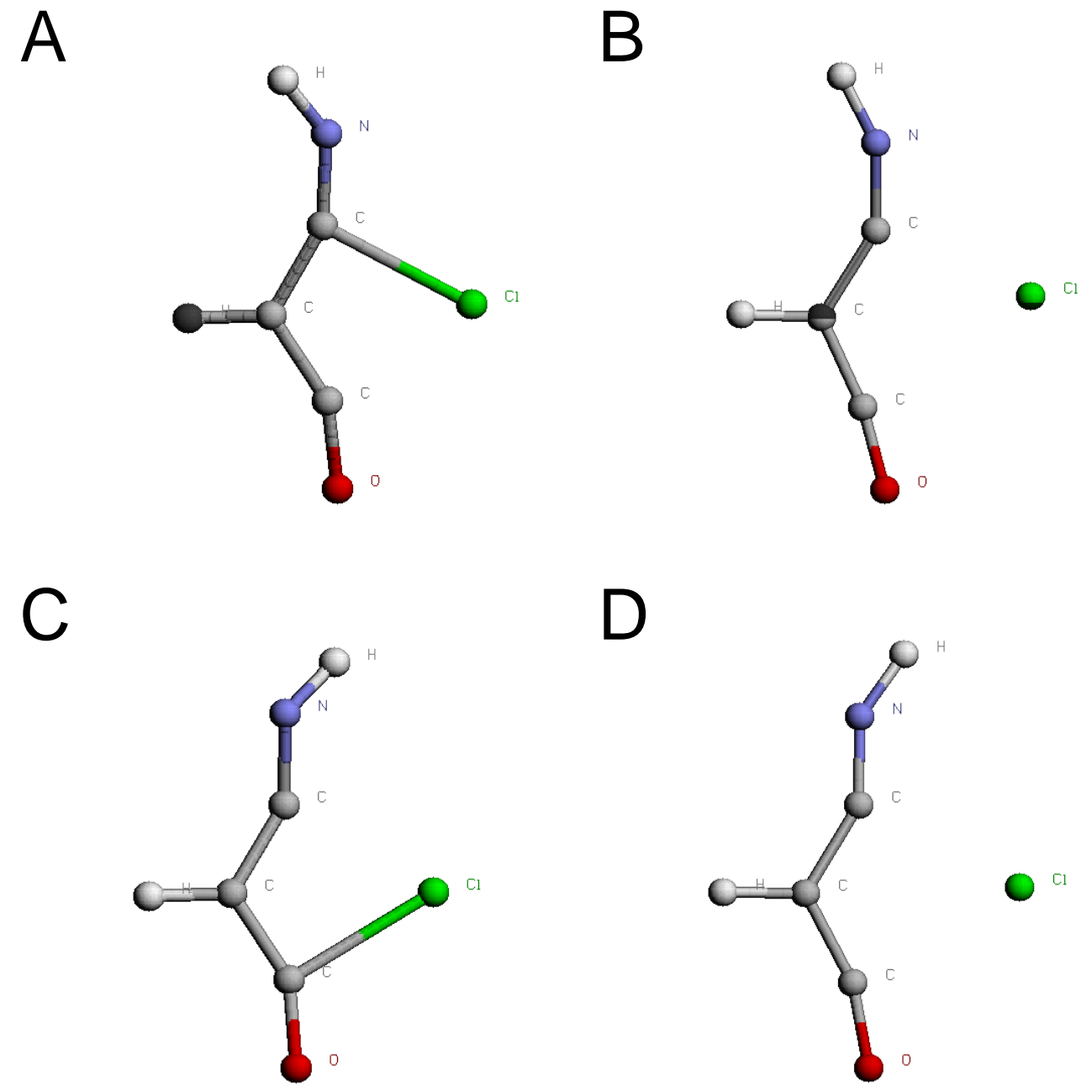

Figure S4 Calculated Cl migration transition states for ketene 1/ketenimine 3 (A and C for the gas phase; B and $\mathrm{D}$ in simulated acetonitrile solvent) 
A

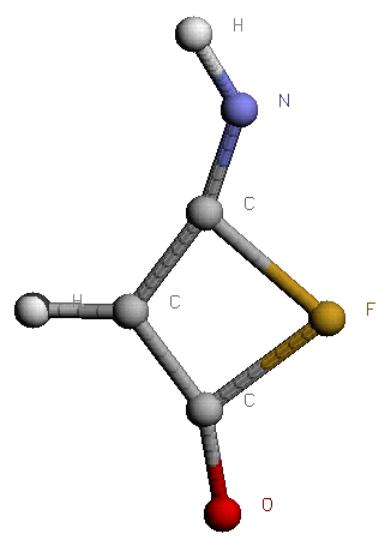

C

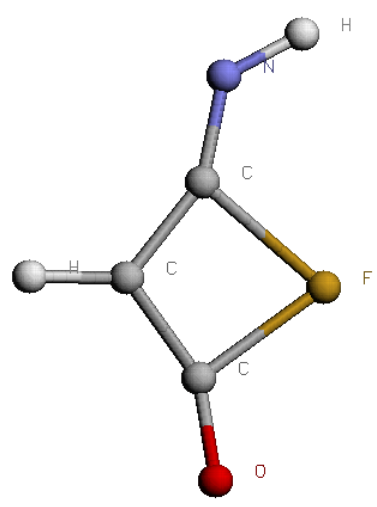

B

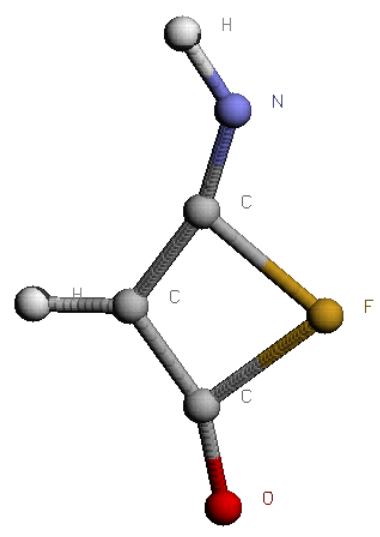

$\mathrm{D}$

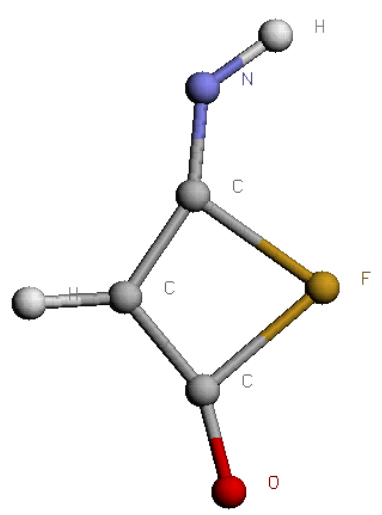

Figure S5 Calculated F migration transition states for ketene 1/ketenimine 3 (A and C for the gas phase; B and $\mathrm{D}$ in simulated acetonitrile solvent) 


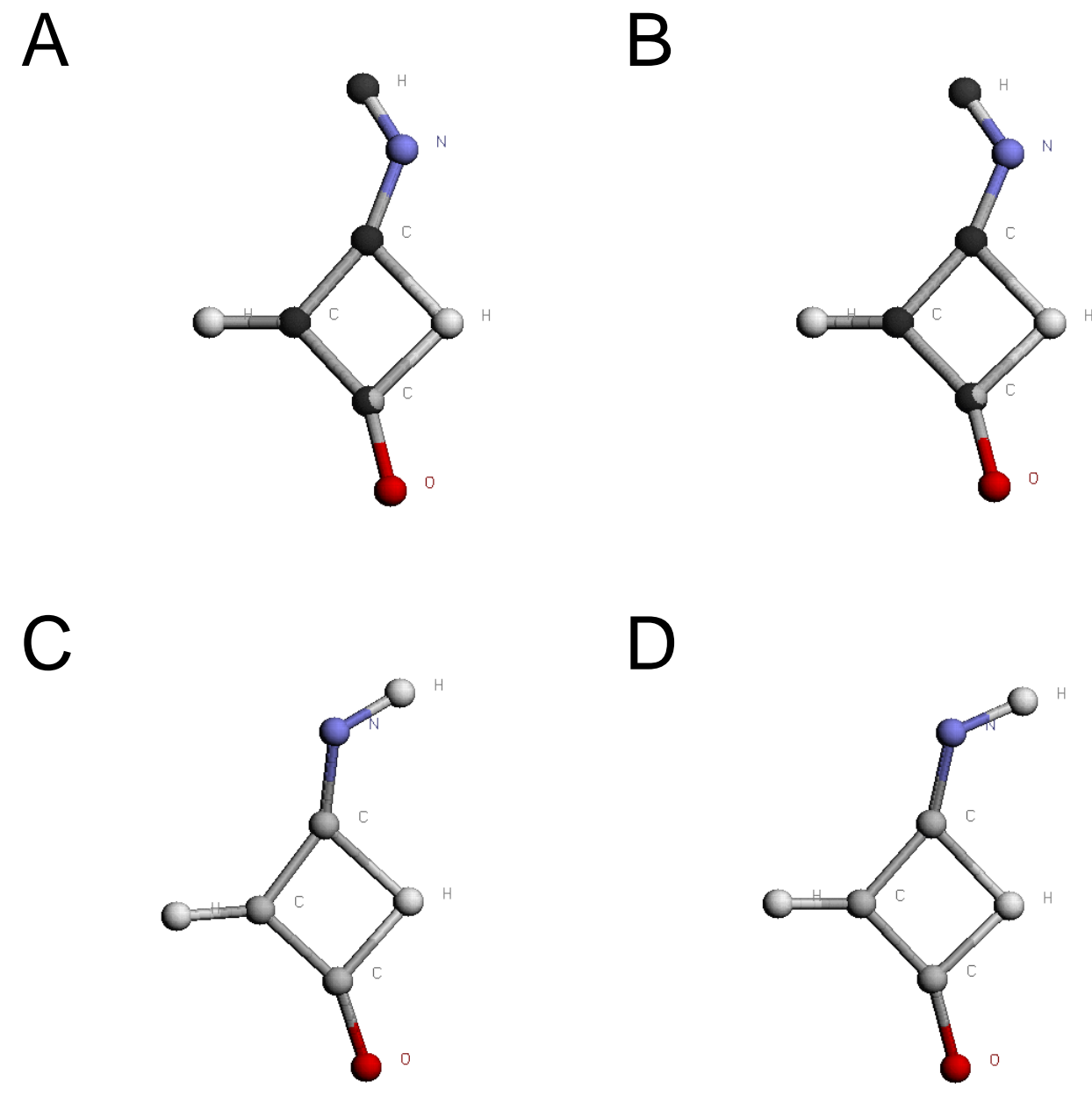

Figure S6 Calculated H migration transition states for ketene 1/ketenimine 3 (A and C for the gas phase; B and D in simulated acetonitrile solvent) 
A

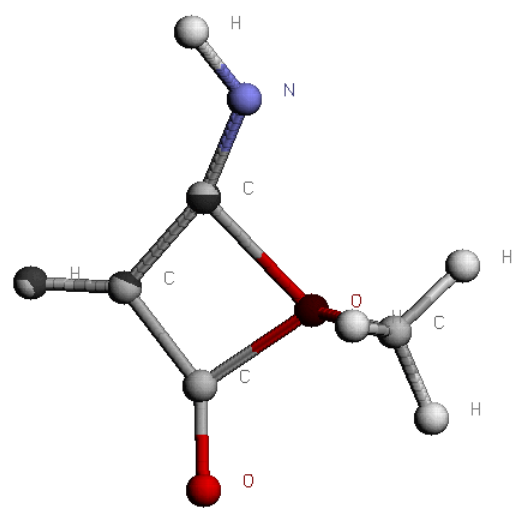

C

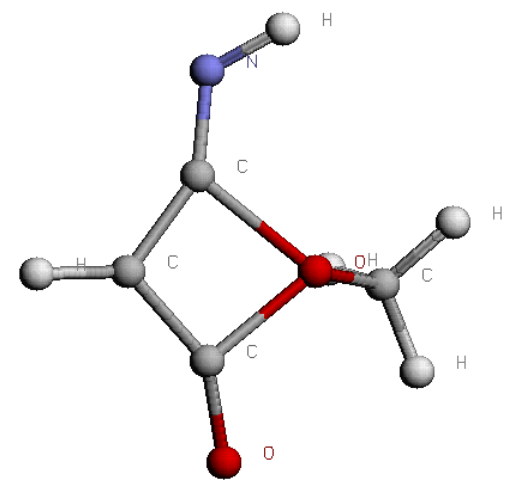

B

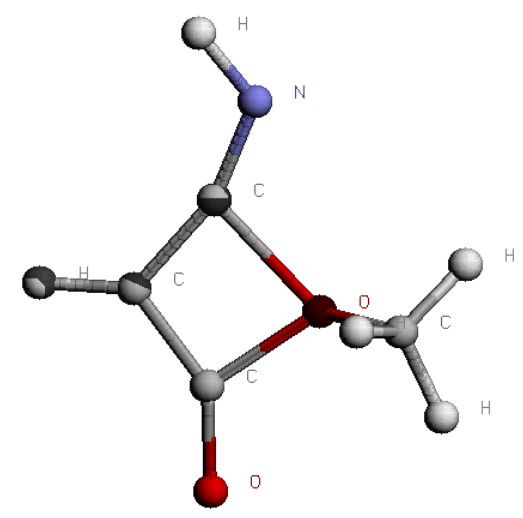

$\mathrm{D}$

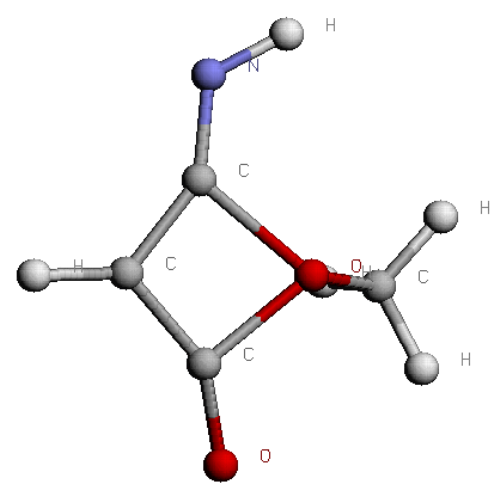

Figure S7 Calculated methoxy migration transition states for ketene $\mathbf{1} /$ ketenimine 3 (A and C for the gas phase; $\mathrm{B}$ and $\mathrm{D}$ in simulated acetonitrile solvent) 
A

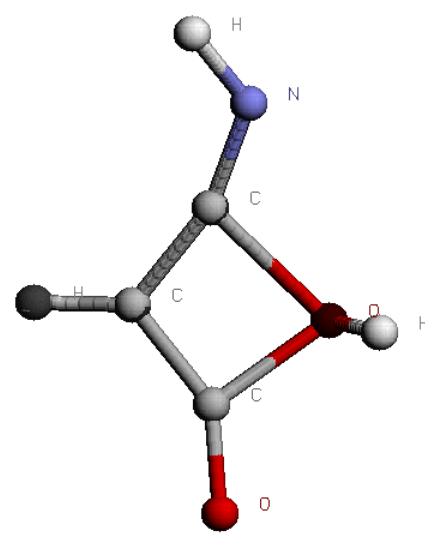

C

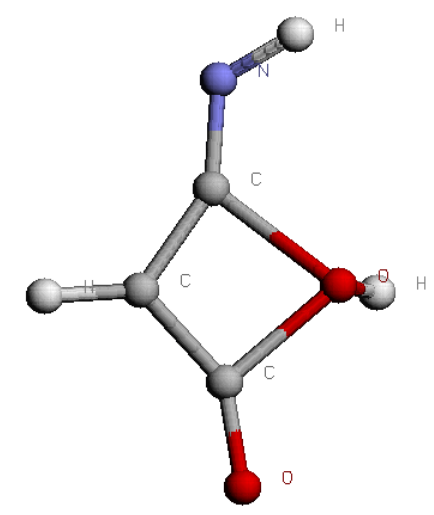

B

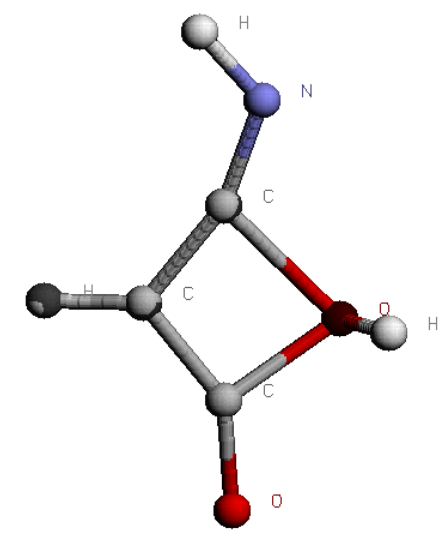

$\mathrm{D}$

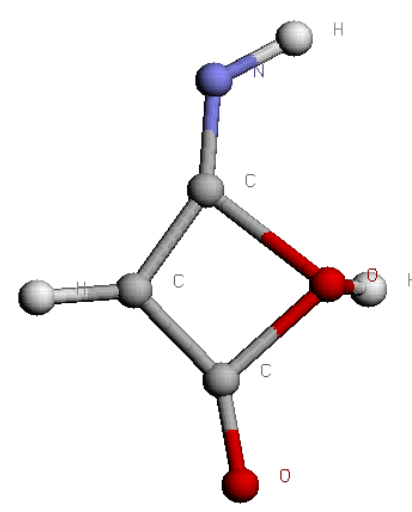

Figure S8 Calculated OH migration transition states (for ketene 1/ketenimine 3 (A and C for the gas phase; B and $\mathrm{D}$ in simulated acetonitrile solvent) 
A $\quad$ B
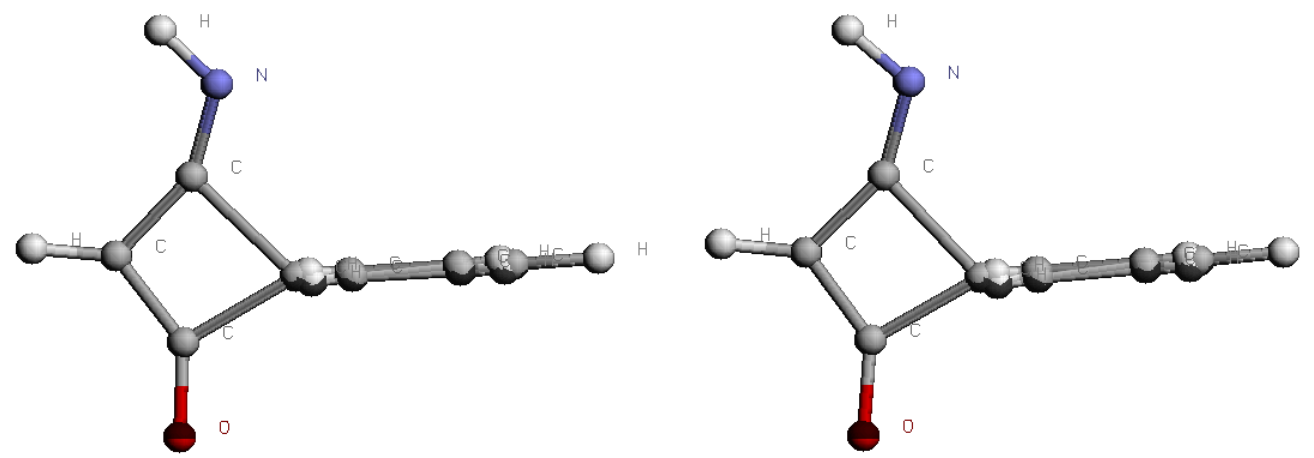

C

$\mathrm{D}$
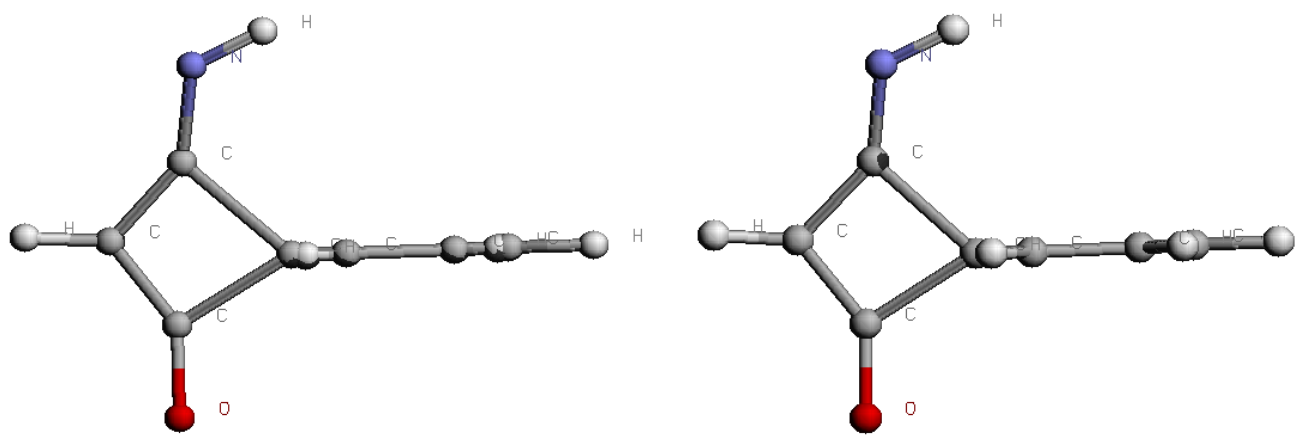

Figure S9 Calculated phenyl migration transition states for ketene 1/ketenimine $\mathbf{3}$ (A and C for the gas phase; B and $\mathrm{D}$ in simulated acetonitrile solvent) 
A

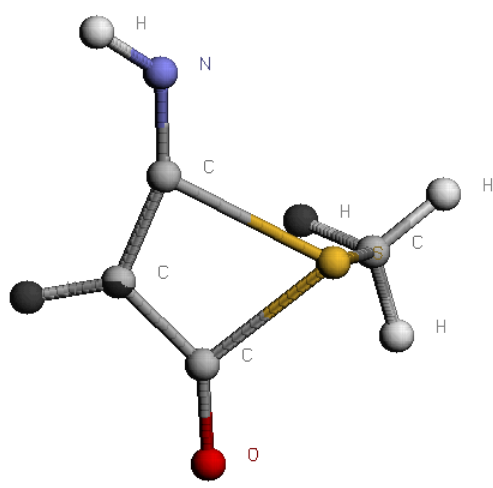

C

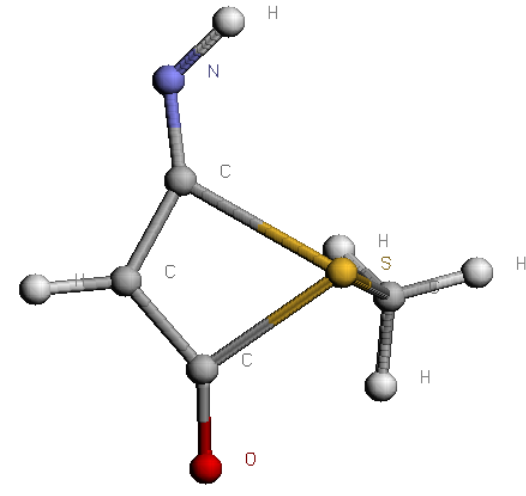

B

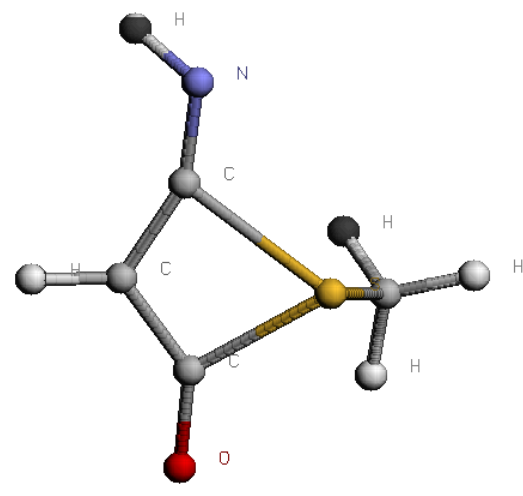

$\mathrm{D}$

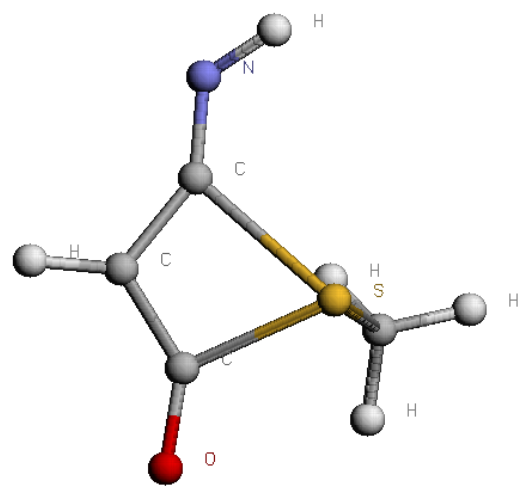

Figure $\mathrm{S} 10$ Calculated $\mathrm{SCH}_{3}$ migration transition states for ketene $\mathbf{1} /$ ketenimine 3 (A and C for the gas phase; $\mathrm{B}$ and $\mathrm{D}$ in simulated acetonitrile solvent) 


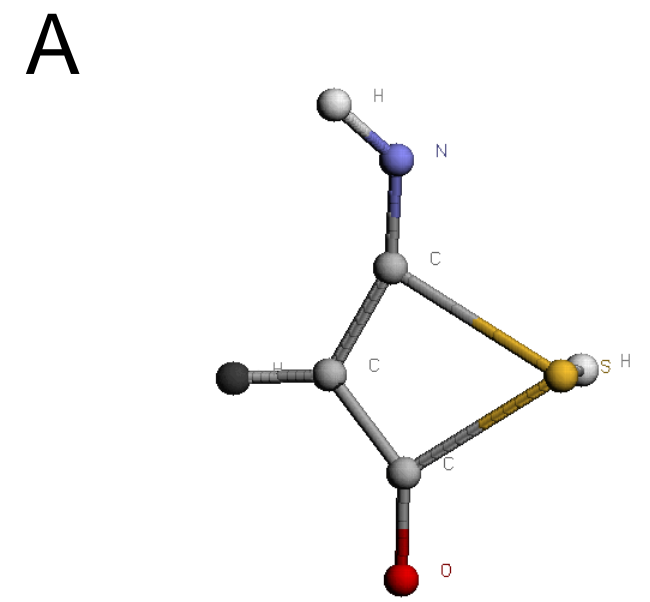

B

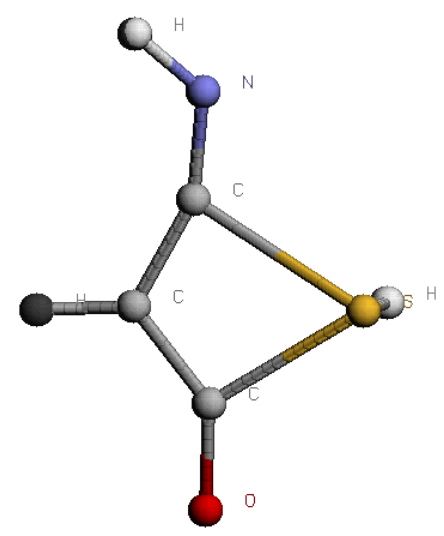

C

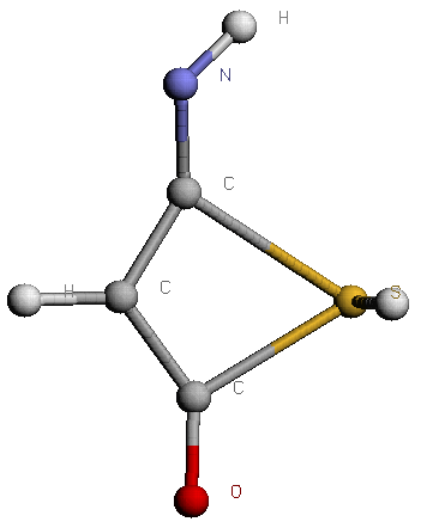

$\mathrm{D}$

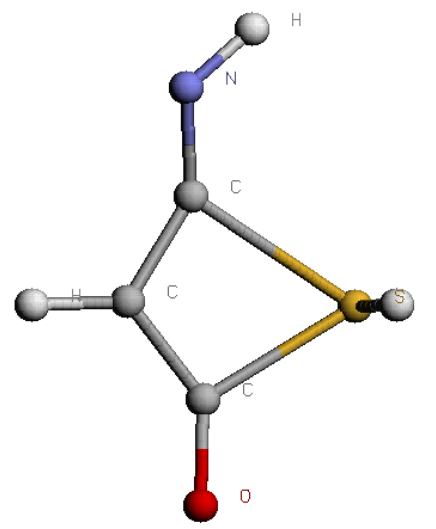

Figure S11 Calculated SH migration transition states for ketene 1/ketenimine 3 (A and C for the gas phase; B and $\mathrm{D}$ in simulated acetonitrile solvent) 
A

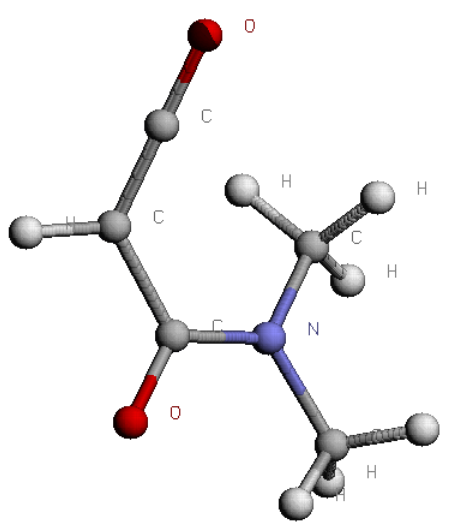

C

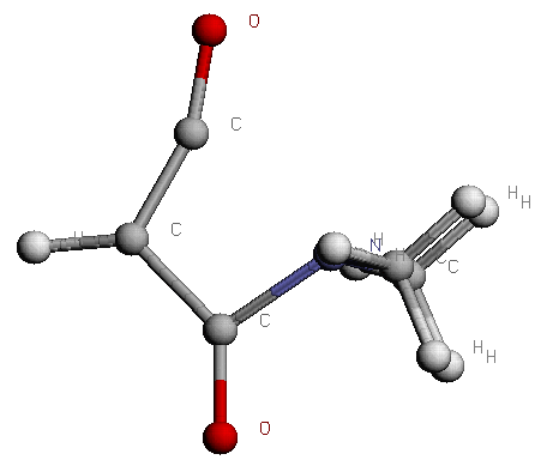

$E$
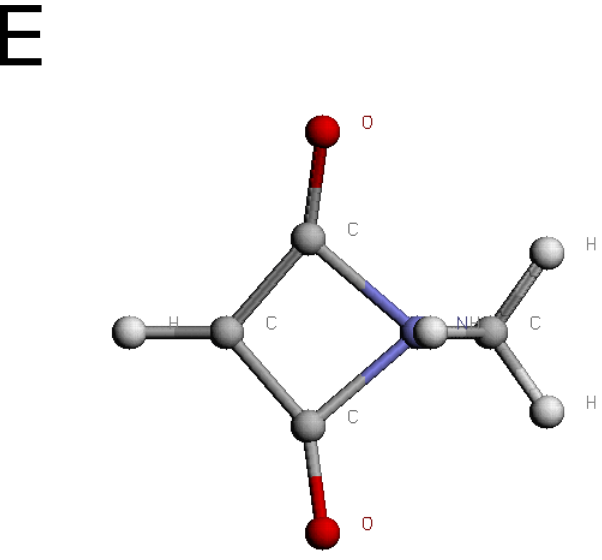

B

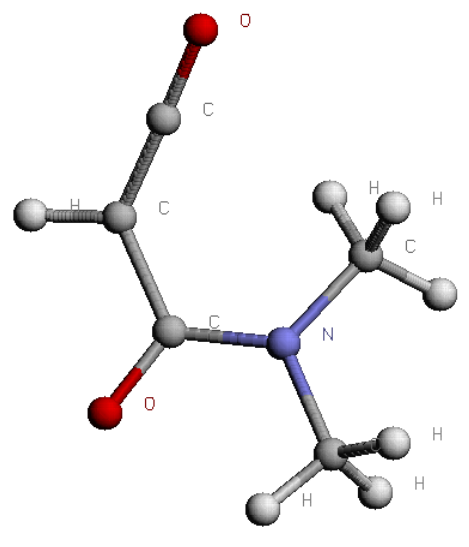

$\mathrm{D}$

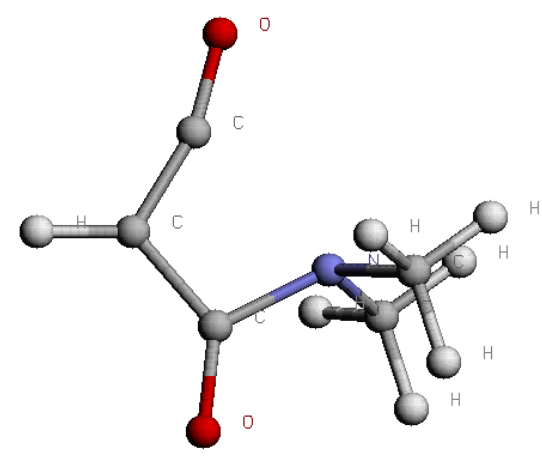

F

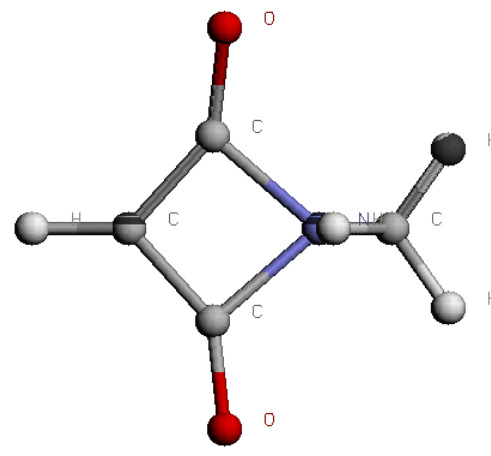

Figure S12 Calculated $\mathrm{N}\left(\mathrm{CH}_{3}\right)_{2}$ migration in $\alpha$-oxoketene 4: A,B ground state, C,D transition state and E,F intermediate (B, D and $\mathrm{F}$ in simulated acetonitrile solvent) 
A

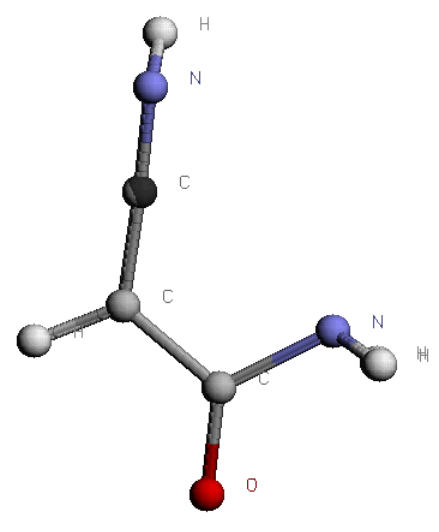

C

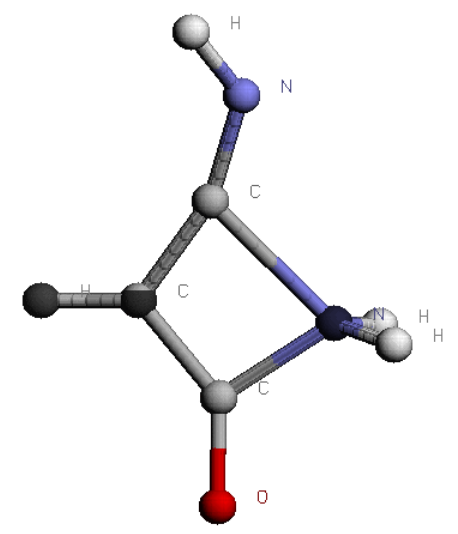

$E$

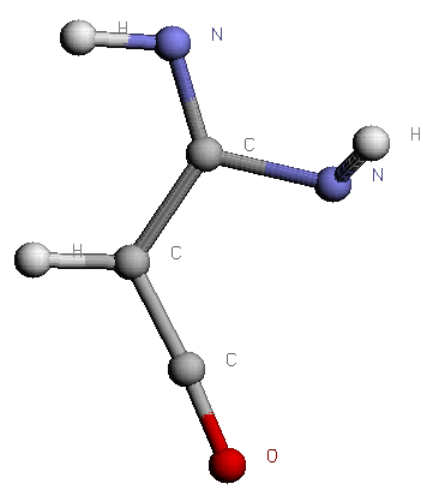

B

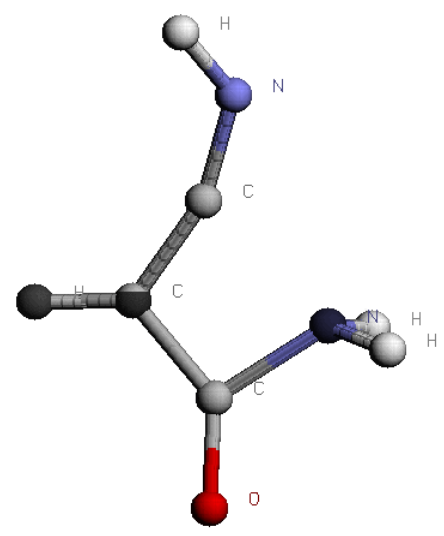

D

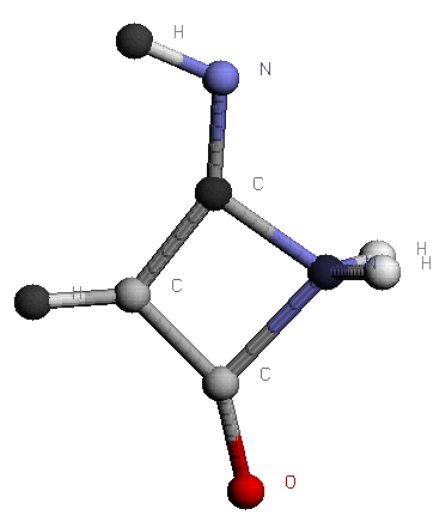

F

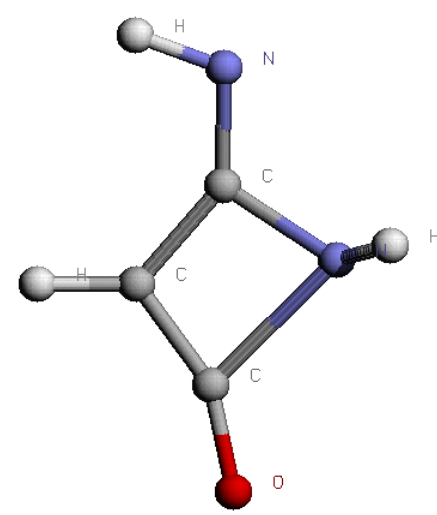

Figure $\mathrm{S} 13$ Calculated $\mathrm{NH}_{2}$ migration for $\mathrm{iE}$ conformer of ketenimine 3/ketene 1: A and $\mathrm{E} \mathrm{NH}_{2}$ rotation transition state and C migration transition state; in simulated solvent $\mathrm{B}$ and $\mathrm{F}$ migration transition states and $\mathrm{D}$ intermediate. 
A

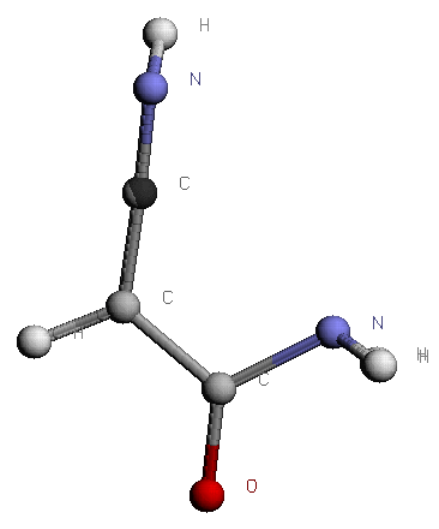

C

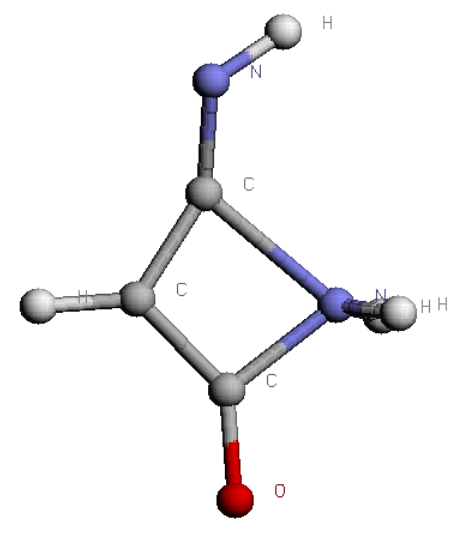

$E$

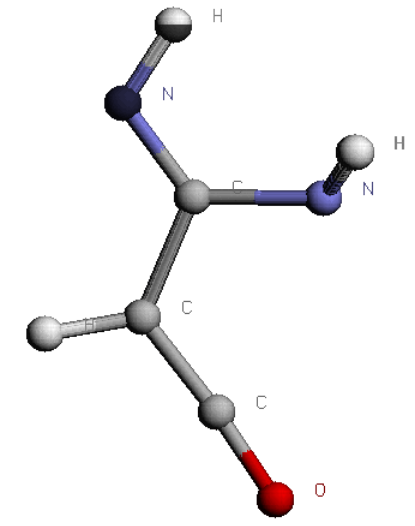

B

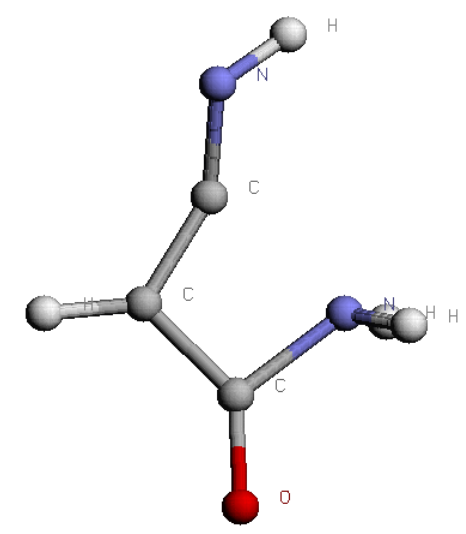

D

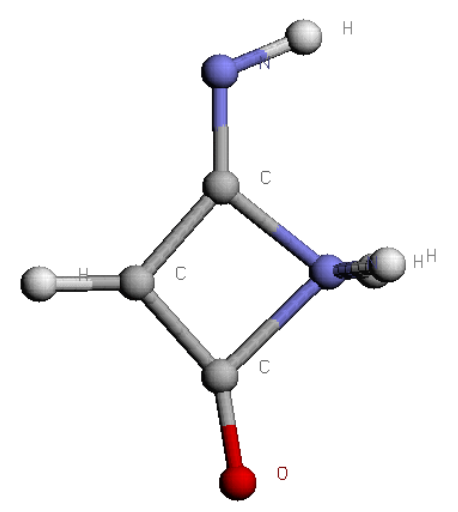

$\mathrm{F}$

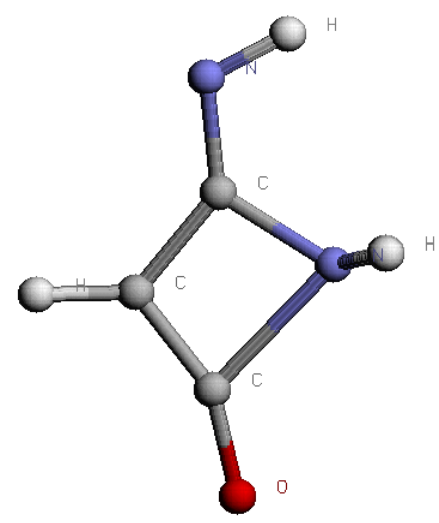

Figure S14 Calculated $\mathrm{NH}_{2}$ migration for $\mathrm{iZ}$ conformer of ketenimine 3 /ketene 1: A and $\mathrm{E} \mathrm{NH}_{2}$ rotation transition state and C migration transition state; in simulated solvent $\mathrm{B}$ and $\mathrm{F}$ migration transition states and $\mathrm{D}$ intermediate 
A

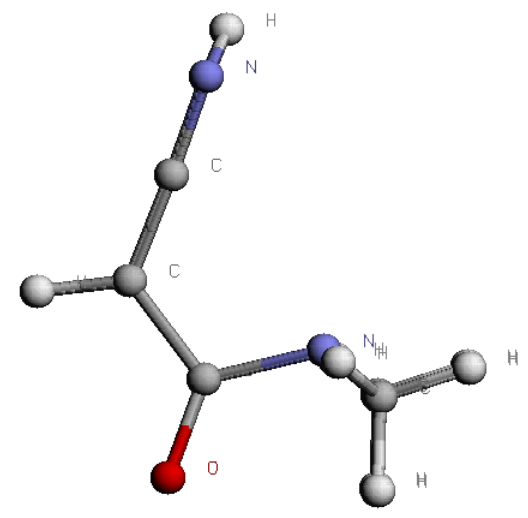

C

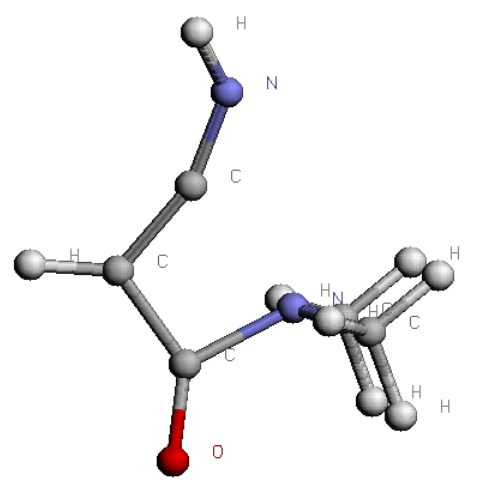

$E$
B

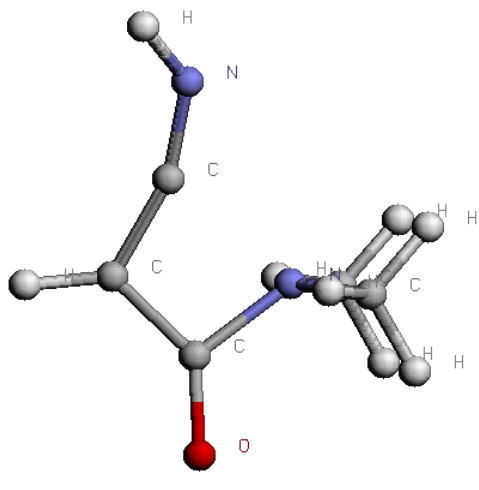

$\mathrm{D}$

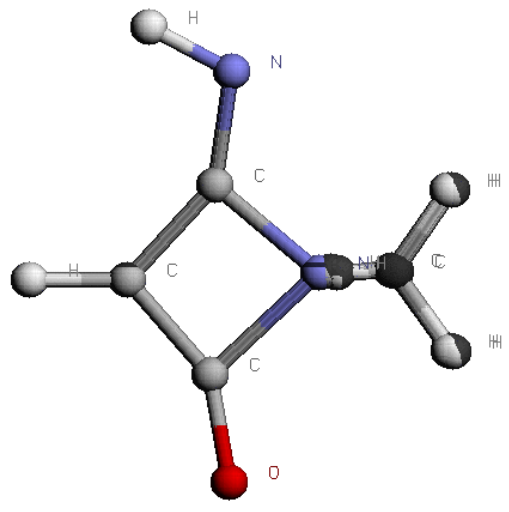

F

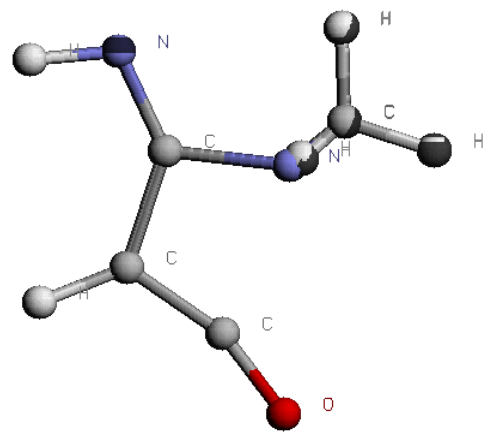

Figure S15 Calculated $\mathrm{N}\left(\mathrm{CH}_{3}\right)_{2}$ migration for $i E$ conformer of of ketenimine $\mathbf{3}$ /ketene $\mathbf{1}$ : A and $\mathrm{E} \mathrm{N}\left(\mathrm{CH}_{3}\right)_{2}$ rotation transition state and $\mathrm{C}$ migration transition state; in simulated solvent $\mathrm{B}$ and $\mathrm{F}$ migration transition states and D intermediate. 
A

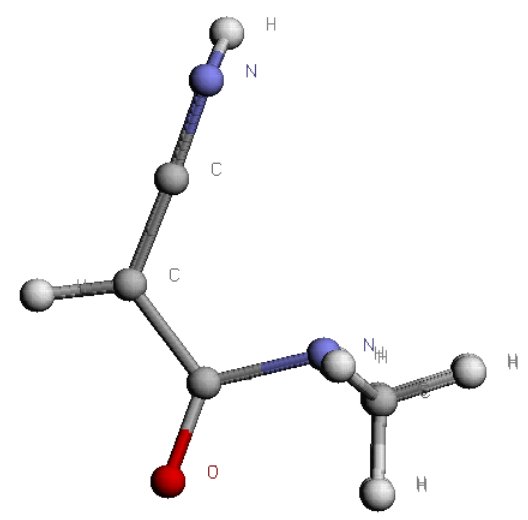

C

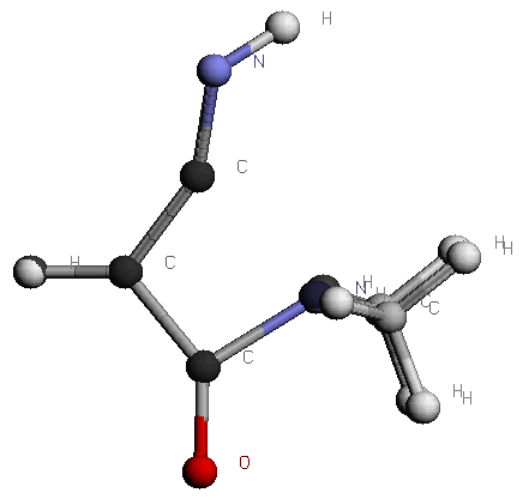

$E$

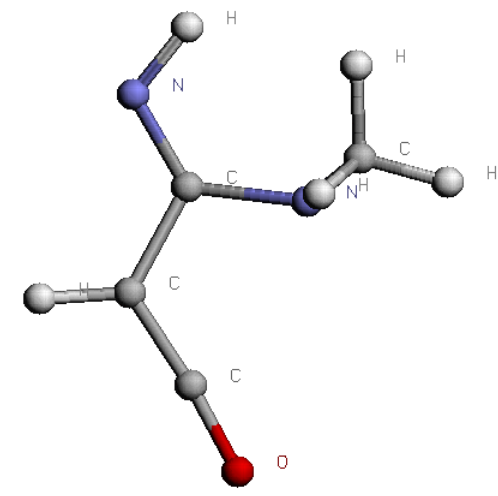

B

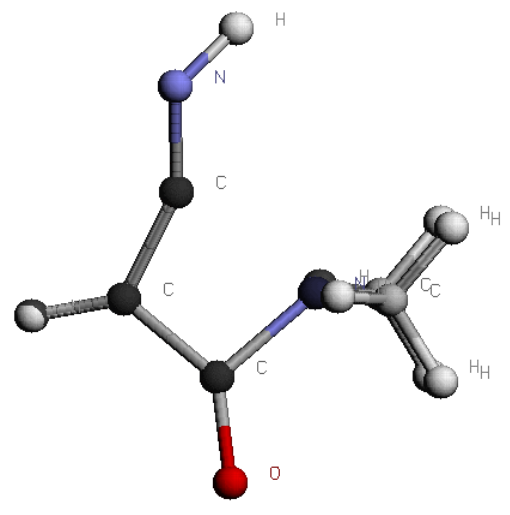

D

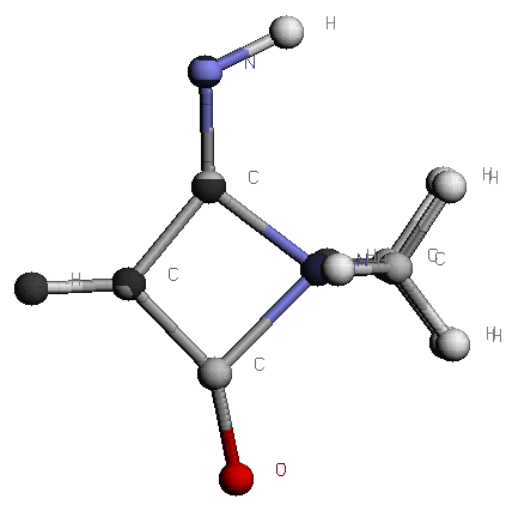

F

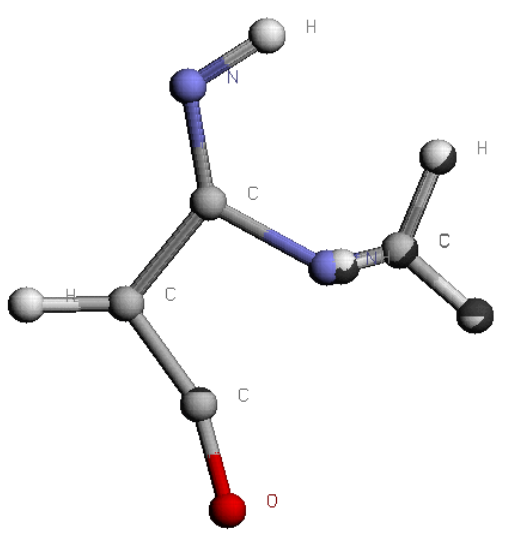

Figure S16 Calculated $\mathrm{N}\left(\mathrm{CH}_{3}\right)_{2}$ migration for $\mathrm{iZ}$ conformer of ketenimine $\mathbf{3} /$ ketene $\mathbf{1}$ : A and E $\mathrm{N}\left(\mathrm{CH}_{3}\right)_{2}$ rotation transition state and C migration transition state; in simulated solvent $\mathrm{B}$ and $\mathrm{F}$ migration transition states and $\mathrm{D}$ intermediate. 
The following data are for the $\alpha$-imidoylketenes 1 - $\alpha$-oxoketenimines 3 interconversion.

\section{1,3 Migration of $\mathrm{Br}$}

\section{Calculation summary for 1,3 Migration of $\mathrm{Br} 3 \mathrm{sZ}$ (vacuum)}

Geometry optimisation and frequency calculation: Program version $=$ x86-Linux-G03RevB.01. Method and basis set $=$ RB3LYP/6-31G(d). Calculated Energy = -2817.1590353 (hartree), $-11781.3590856246\left(\mathrm{~kJ} \mathrm{~mol}^{-1}\right)$. Higher level single-point calculation: Program = x86-Linux-G03RevB.01. Method and basis set $=$ RB3LYP/6$311+G(3 d f, 2 p)$. Calculated Energy $=-2819.6992614$ (hartree), $-11791.9823111748\left(\mathrm{~kJ} \mathrm{~mol}^{-1}\right)$. Structure definition (3D Coordinates: Atom, X coord/Å, Y coord/Å, Z coord/ $\AA$ ): C, 0.8675463022, 0.0285502773, 1.0211730812; C, 0.8873959481, 0.0117000679, 2.3540891133; H, 1.7929822332, 0.0711447653, 0.4619925848; N, 0.8966828011, 0.1268644538, 3.5598072117; C, -0.4110706445, -0.0324651539, 0.3305767291; O, -1.5108604419, -0.1049951748, 0.7885920166; Br, -0.1421032508, 0.0150367194, 1.6597701729; H, 0.9275058379, -0.6922308713, 4.167543311. Harmonic Frequencies (Number, Frequency $\left(\mathrm{cm}^{-1}\right)$, IR Intensity $\left.\left(\mathrm{km} \mathrm{mol}^{-1}\right)\right): 1$, 80.2614, 2.8191; 2, 131.0686, 1.4356; 3, 253.7595, 11.0555; 4, 337.8308, 11.9319; 5, 468.1704, 16.9332; 6, 481.4989, 65.1179; 7, 508.7723, 38.1884; 8, 683.3831, 34.2866; 9, 799.3256, 325.0783; 10, 843.0486, 64.6159; 11, 901.7795, 474.7373; 12, 1020.1, 154.9410; 13, 1155.3897, 6.2000; 14, 1410.3065, 159.4218; 15, 1867.4776, 267.1667; 16, 2151.1688, 687.9603; 17, 3256.9415, 11.8301; 18, 3510.0629, 64.8703;

\section{Calculation summary for 1,3 Migration of $\mathrm{Br} 3 \mathrm{sE}$ (vacuum)}

Geometry optimisation and frequency calculation: Program version = x86-Linux-G03RevB.01. Method and basis set $=$ RB3LYP/6-31G(d). Calculated Energy = -2817.1601812 (hartree), $-11781.3638777784\left(\mathrm{~kJ} \mathrm{~mol}^{-1}\right)$. Higher level single-point calculation: Program = x86-Linux-G03RevB.01. Method and basis set $=$ RB3LYP/6$311+G(3 d f, 2 p)$. Calculated Energy = -2819.6998146 (hartree), $-11791.9846246572\left(\mathrm{~kJ} \mathrm{~mol}^{-1}\right)$. Structure definition (3D Coordinates: Atom, X coord/Å, Y coord/Å, Z coord/Å): C, 1.542392542, 0.1635505205, 0.6679132978; C, 1.90224605, 0.039689168, 0.6086768497; N, 2.2912730093, 0.0584504114, 1.755124116; C, 0.1715337165, 0.1492474573, -1.1365877302; Br, -1.1685957798, -0.1176790064, 0.3470720589; O, 0.2050009747, 0.2660852844, -2.2617794874; H, 2.3148937483, 0.2713264804, -1.4240651004; H, 2.4900214255, -0.8053192855, 2.2598551946. Harmonic Frequencies (Number, Frequency $\left(\mathrm{cm}^{-1}\right.$ ), IR Intensity $\left.\left(\mathrm{km} \mathrm{mol}^{-1}\right)\right): 1$, 111.475, 3.7833; 2, 124.1646, 0.0401; 3, 299.2124, 7.9081; 4, 329.9666, 3.9177; 5, 452.8091, 42.0597; 6, 506.8315, 31.5197; 7, 546.3028, 106.3307; 8, 597.2371, 5.5086; 9, 699.9358, 46.0285; 10 , 834.0485, 73.4343; 11, 896.7985, 439.9537; 12, 1077.5742, 185.8012; 13, 1178.1669, 79.1516; 14, 1413.9887, 16.6395; 15, 1880.0521, 524.6801; 16, 2157.6097, 480.9471; 17, 3207.4217, 5.0391; 18, 3516.4294, 64.5407;

\section{Calculation summary for 1,3 Migration of Br TS2 iE (vacuum)}

Geometry optimisation and frequency calculation: Program version $=$ x86-Linux-G03RevB.01. Method and basis set = RB3LYP/6-31G(d). Calculated Energy = -2817.1290018 (hartree), $-11781.2334855276\left(\mathrm{~kJ} \mathrm{~mol}^{-1}\right)$. Higher level single-point calculation: Program = x86-Linux-G03RevB.01. Method and basis set $=$ RB3LYP/6311+G(3df,2p). Calculated Energy $=-2819.6685724$ (hartree), $-11791.8539697768\left(\mathrm{~kJ} \mathrm{~mol}^{-1}\right)$. Structure definition (3D Coordinates: Atom, X coord/A, Y coord/A, Z coord/ $\AA$ ): C, 1.2285113199, 0.0084073255, 1.1129424299; C, 1.4958824641, 0.0485651613, 0.23823966; O, 2.2758741346, 0.1070086109, 1.1099397137; C, -0.1111629609, -0.0838529995, -1.4869634922; N, -0.9834307201, -0.1483346146, -2.322604136; Br, 0.801581514, 0.023937703, 0.7610454663; H, 2.0296237881, 0.0718157576, -1.8449171111; H, 0.9766337735, -0.5660788718, -3.2429653941. Harmonic Frequencies (Number, Frequency $\left(\mathrm{cm}^{-1}\right)$, IR 
Intensity ( $\left.\mathrm{km} \mathrm{mol}^{-1}\right)$ ): 1, -421.2672, 135.5112; 2, 137.5937, 4.3259; 3, 186.9583, 24.9390; 4, 298.804, 33.2104; 5, 367.961, 127.8238; 6, 398.9953, 174.6374; 7, 508.0302, 64.5546; 8, 534.6167, 4.9337; 9, 570.5898, 41.3334; 10, 635.5844, 103.2891; 11, 706.5885, 138.3557; 12, 1093.461, 151.0111; 13, 1103.3137, 40.7598; 14, 1395.8106, 4.0191; 15, 2030.2504, 1293.2606; 16, 2103.8255, 235.2306; 17, 3196.5637, 0.0997; 18, 3642.0362, 199.8291;

\section{Calculation summary for 1,3 Migration of Br TS2 iZ (vacuum)}

Geometry optimisation and frequency calculation: Program version = x86-Linux-G03RevB.01. Method and basis set = RB3LYP/6-31G(d). Calculated Energy = -2817.1394978 (hartree), $-11781.2773797996\left(\mathrm{~kJ} \mathrm{~mol}^{-1}\right)$. Higher level single-point calculation: Program = x86-Linux-G03RevB.01. Method and basis set $=$ RB3LYP/6311+G(3df,2p). Calculated Energy = -2819.6782979 (hartree), $-11791.8946418178\left(\mathrm{~kJ} \mathrm{~mol}^{-1}\right)$. Structure definition (3D Coordinates: Atom, X coord/Å, Y coord/Å, Z coord/Å): C, 1.4374756673, 0.1171698494, 0.8653310974; C, 1.4389886707, 0.0432181843, 0.5203151534; N, 2.0792639028, 0.0972588371, 1.5470447958; C, 0.2004018728, 0.1272075774, -1.4766856459; Br, -0.9691785622, -0.1046174675, 0.5387237082; O, -0.4153930547, 0.1705473638, -2.4727545061; H, 2.357344921, 0.1431226557, 1.4422678332; H, 1.8710046088, -0.2522757283, 2.4698700638. Harmonic Frequencies (Number, Frequency $\left(\mathrm{cm}^{-1}\right)$, IR Intensity $\left.\left(\mathrm{km} \mathrm{mol}^{-1}\right)\right): 1,-353.8161,114.0685 ; 2,136.0332,1.4685 ; 3,203.0061,31.6570 ; 4$, 284.6366, 82.0817; 5, 352.2866, 44.1209; 6, 421.8823, 159.4054; 7, 521.6142, 9.6329; 8, 556.7468, 52.7103; 9, 582.5097, 7.7510; 10, 679.548, 70.6379; 11, 742.351, 479.5562; 12, 1088.0435, 130.3035; 13, 1104.2262, 103.6483; 14, 1401.051, 6.5619; 15, 2035.2448, 1161.8460; 16, 2098.948, 195.4445; 17, 3208.5007, 1.3974; 18, 3684.4835, 230.4107;

\section{Calculation summary for 1,3 Migration of $\operatorname{Br} 1 \mathrm{sE}$ iE (vacuum)}

Geometry optimisation and frequency calculation: Program version = x86-Linux-G03RevB.01. Method and basis set = RB3LYP/6-31G(d). Calculated Energy = -2817.1518933 (hartree), $-11781.3292177806\left(\mathrm{~kJ} \mathrm{~mol}^{-1}\right)$. Higher level single-point calculation: Program = x86-Linux-G03RevB.01. Method and basis set $=$ RB3LYP/6311+G(3df,2p). Calculated Energy = -2819.6917975 (hartree), -11791.951097145 (kJ mol $\left.{ }^{-1}\right)$. Structure definition (3D Coordinates: Atom, X coord/Å, Y coord/Å, Z coord/Å): C, 1.6323619205, 0.2239840612, 0.1711982703; C, 1.6241855156, -0.0189826865, 1.1384653442; O, 1.6866855998, -0.2226573866, 2.280951685; C, 0.4339542902, 0.2351678014, -0.9962047062; N, 0.298424311, 0.4472090023, 2.2310306175; Br, -1.1864462422, -0.1442371723, 0.0242157446; H, 2.6010904118, 0.4215771124, 0.6225710527; H, 1.1990627316, 0.6365049371, -2.68175337. Harmonic Frequencies (Number, Frequency $\left(\mathrm{cm}^{-1}\right)$, IR Intensity $\left.\left(\mathrm{km} \mathrm{mol}^{-1}\right)\right): 1,106.7877,3.9058 ; 2,120.347,0.5038 ; 3,318.1962,7.5978 ; 4,357.129$, 0.3896; 5, 486.9735, 7.1543; 6, 534.063, 65.1953; 7, 543.4321, 17.9614; 8, 617.5743, 0.4368; 9, 632.9519, 9.5353; 10, 827.629, 109.3716; 11, 1006.0394, 113.9860; 12, 1140.6715, 27.3282; 13, 1245.9812, 199.7857; 14, 1403.8985, 11.2239; 15, 1725.926, 320.1267; 16, 2237.197, 737.0670; 17, 3197.8016, 4.7906; 18, 3437.4277, 1.8416;

\section{Calculation summary for 1,3 Migration of $\mathrm{Br} 1 \mathrm{sE}$ iZ (vacuum)}

Geometry optimisation and frequency calculation: Program version = x86-Linux-G03RevB.01. Method and basis set = RB3LYP/6-31G(d). Calculated Energy = -2817.1590329 (hartree), $-11781.3590755878\left(\mathrm{~kJ} \mathrm{~mol}^{-1}\right)$. Higher level single-point calculation: Program = x86-Linux-G03RevB.01. Method and basis set = RB3LYP/6311+G(3df,2p). Calculated Energy = -2819.6982776 (hartree), -11791.9781969232 (kJ mol $\left.{ }^{-1}\right)$. Structure definition (3D Coordinates: Atom, X coord/A, Y coord/Å, Z coord/Å): C, 1.6547507718, -0.0444701236, 0.2696601236; C, 0.4349546791, -0.0251284354, -1.0473390355; N, 0.3942598893, -0.0415643467, - 
2.3024923084; C, 1.6901070992, -0.0268725304, 1.0604909632; Br, -1.1968918786, 0.0307568487, 0.0972796845; O, 1.7993242515, -0.013460685, 2.2176393983; H, 2.6040572246, -0.0753605077, 0.7962104898; H, -0.5461300113, -0.0236667534, -2.6931983196. Harmonic Frequencies (Number, Frequency $\left(\mathrm{cm}^{-1}\right)$, IR Intensity $\left.\left(\mathrm{km} \mathrm{mol}^{-1}\right)\right): 1,112.3034,0.8681 ; 2,116.7355,0.2986 ; 3,312.1811,19.2523 ; 4,332.5846$, 10.3187; 5, 484.4707, 13.8177; 6, 517.9502, 48.7603; 7, 538.3594, 11.0825; 8, 621.8694, 1.7536; 9, 663.2776, 76.3879; 10, 802.6214, 50.1857; 11, 1015.8663, 121.9420; 12, 1129.4817, 30.8459; 13, 1205.3826, 302.8167; 14, 1414.7575, 17.4120; 15, 1762.0199, 311.6602; 16, 2241.301, 733.4146; 17, 3212.6046, 13.9838; 18, 3531.1995, 30.9122;

\section{Calculation summary for $\mathbf{1 , 3}$ Migration of $\mathrm{Br} 1 \mathrm{sZ}$ iE (vacuum)}

Geometry optimisation and frequency calculation: Program version $=$ x86-Linux-G03RevB.01. Method and basis set = RB3LYP/6-31G(d). Calculated Energy = -2817.1473046 (hartree), -11781.3100278372 (kJ mol ${ }^{-1}$ ). Higher level single-point calculation: Program = x86-Linux-G03RevB.01. Method and basis set = RB3LYP/6$311+\mathrm{G}(3 \mathrm{df}, 2 \mathrm{p})$. Calculated Energy = -2819.688195 (hartree), -11791.93603149 (kJ mol$\left.{ }^{-1}\right)$. Structure definition (3D Coordinates: Atom, X coord/Å, Y coord/Å, Z coord/Å): C, 0.8799693517, 0.0987351633, 0.9926489663; C, 0.9691803647, 0.1186838544, 2.3177613219; H, 1.8020113556, 0.1896965194, 0.4311918086; O, 1.0314846261, 0.134503998, 3.4804034154; C, -0.4100406541, -0.0402220015, 0.3075449366; N, 1.5914473754, -0.1592624497, 0.7320418003; Br, -0.1686872425, -0.0302071458, -1.6246358391; H, 1.6443576239, -0.1568233493, 1.7558112851. Harmonic Frequencies (Number, Frequency ( $\mathrm{cm}^{-1}$ ), IR Intensity $\left(\mathrm{km} \mathrm{mol}^{-1}\right)$ ): 1, 41.0977, 6.4695; 2, 125.1318, 4.4851; 3, 259.8269, 2.6294; 4, 370.3363, 0.8805; 5, 501.4648, 21.5211; 6, 506.1672, 23.0263; 7, 532.5093, 7.7005; 8, 629.7239, 16.9492; 9, 754.8687, 174.6120; 10, 810.0534, 83.9808; 11, 975.434, 63.1081; 12, 1147.6715, 7.6031; 13, 1190.5157, 131.3588; 14, 1408.2776, 151.9848; 15, 1711.9556, 191.8792; 16, 2224.7574, 788.5065; 17, 3239.6487, 19.9482; 18, 3427.6376, 2.9373;

\section{Calculation summary for 1,3 Migration of $\mathrm{Br} 1 \mathrm{sZ}$ iZ (vacuum)}

Geometry optimisation and frequency calculation: Program version = x86-Linux-G03RevB.01. Method and basis set = RB3LYP/6-31G(d). Calculated Energy = -2817.157346 (hartree), -11781.352020972 (kJ mol $\left.{ }^{-1}\right)$. Higher level single-point calculation: Program = x86-Linux-G03RevB.01. Method and basis set = RB3LYP/6$311+G(3 d f, 2 p)$. Calculated Energy = -2819.6972621 (hartree), $-11791.9739501022\left(\mathrm{~kJ} \mathrm{~mol}^{-1}\right)$. Structure definition (3D Coordinates: Atom, X coord/A, Y coord/Å, Z coord/Å): C, 0.8865713964, 0.0537466494, 1.020076634; C, 0.921454637, 0.0273603579, 2.3512625427; H, 1.8227425122, 0.1458097905, 0.4857311978; O, 0.9750117844, 0.0063095211, 3.5111785122; C, -0.3894852709, -0.0403030778, 0.3293783412; N, 1.5120426373, -0.14837184, 0.8876758323; Br, -0.1499972178, 0.0228382652, -1.6353758303; H, 2.2998802788, -0.2018459402, 0.2449588305. Harmonic Frequencies (Number, Frequency ( $\mathrm{cm}^{-1}$ ), IR Intensity $\left.\left(\mathrm{km} \mathrm{mol}^{-1}\right)\right): 1,67.8281,0.2301 ; 2,131.4288,1.1143 ; 3,254.1277,4.6452 ; 4,341.5458,19.3980 ; 5,480.708$, 27.1865; 6, 514.9138, 13.1854; 7, 535.3021, 8.8216; 8, 636.8049, 43.8329; 9, 771.6822, 117.7447; 10, 787.8031, 85.5121; 11, 1013.1866, 37.8627; 12, 1149.032, 4.1943; 13, 1168.6106, 350.8837; 14, 1421.9116, 183.4456; 15, 1743.3657, 186.2228; 16, 2238.379, 824.4519; 17, 3258.1528, 19.7824; 18, 3534.2152, 26.3360;

\section{Calculation summary for 1,3 Migration of $\mathrm{Br} 3 \mathrm{sE}$ (acetonitrile)}

Geometry optimisation and frequency calculation: Program version = x86-Linux-G03RevB.01. Method and basis set = RB3LYP/6-31G(d). Calculated Energy = -2817.1770412 (hartree), $-11781.4343862984\left(\mathrm{~kJ} \mathrm{~mol}^{-1}\right)$. Higher level single-point calculation: Program = x86-Linux-G03RevB.01. Method and basis set $=$ RB3LYP/6311++G(3df,2p). Calculated Energy = -2819.7170569 (hartree), -11792.0567319558 $\left(\mathrm{kJ} \mathrm{mol}^{-1}\right)$. Structure 
definition (3D Coordinates: Atom, X coord/Å, Y coord/A, Z coord/Å): C, 1.5490825607, 0.1645580599, 0.6777457672; C, 1.905814103, 0.0300177673, 0.6069087396; N, 2.2849402898, 0.0412058105, 1.7477326633; C, 0.1964076639, 0.1443814746, -1.1462379747; Br, -1.1730424271, -0.1089336995, 0.3522731737; O, -0.2125270085, 0.2523995665, -2.2654514784; H, 2.3324173485, 0.2831993746, 1.4281832008; H, 2.5218756747, -0.8119009098, 2.2905553198. Harmonic Frequencies (Number, Frequency $\left(\mathrm{cm}^{-1}\right)$, IR Intensity $\left.\left(\mathrm{km} \mathrm{mol}^{-1}\right)\right): 1,96.3265,5.5373 ; 2,121.9418,0.4091 ; 3,285.8836,39.3611 ; 4,326.3682$, 10.3351; 5, 453.0019, 95.4678; 6, 502.9999, 117.4675; 7, 524.2662, 235.5699; 8, 596.8942, 15.0632; 9, 685.9694, 74.6306; 10, 771.6192, 872.0225; 11, 793.6277, 127.9780; 12, 1065.836, 321.1955; 13, 1167.284, 131.3793; 14, 1417.4633, 60.2591; 15, 1825.73, 1433.3075; 16, 2158.0064, 858.3670; 17, 3118.6479, 83.4333; 18, 3152.787, 681.8137;

\section{Calculation summary for 1,3 Migration of $\mathrm{Br} 3$ sZ (acetonitrile)}

Geometry optimisation and frequency calculation: Program version = x86-Linux-G03RevB.01. Method and basis set = RB3LYP/6-31G(d). Calculated Energy = -2817.1760428 (hartree), $-11781.4302109896\left(\mathrm{~kJ} \mathrm{~mol}^{-1}\right)$. Higher level single-point calculation: Program = x86-Linux-G03RevB.01. Method and basis set $=$ RB3LYP/6311++G(3df,2p). Calculated Energy = -2819.7165143 (hartree), -11792.0544628026 (kJ mol $\left.{ }^{-1}\right)$. Structure definition (3D Coordinates: Atom, X coord/Å, Y coord/Å, Z coord/Å): C, 0.8630580553, 0.0326435329, 1.0259319734; C, 0.884689379, 0.0070573049, 2.3683682537; H, 1.7958667761, 0.0814837434, 0.4716495363; N, 0.8976376457, 0.1150192943, 3.5652258834; C, -0.4038714389, -0.028124605, 0.3547896473; O, -1.5206391407, -0.0938975813, 0.7820802744; Br, -0.1407498231, 0.0137457679, 1.6686892845; H, 0.9487706649, -0.6859974272, 4.2247127975. Harmonic Frequencies (Number, Frequency $\left(\mathrm{cm}^{-1}\right)$, IR Intensity $\left.\left(\mathrm{km} \mathrm{mol}^{-1}\right)\right): 1$, 44.4813, 3.6077; 2, 125.5788, 4.8813; 3, 243.6181, 63.7610; 4, 326.211, 38.9239; 5, 465.6185, 92.9212; 6, 480.5669, 163.1456; 7, 509.1726, 73.4521; 8, 666.5853, 86.5691; 9, 750.9922, 1493.9089; 10, 775.927, 67.7484; 11, 798.916, 101.0391; 12, 1031.4789, 243.1118; 13, 1154.2721, 41.4468; 14, 1404.4397, 420.2117; 15, 1820.0637, 606.8746; 16, 2151.4477, 1272.8302; 17, 3147.7024, 809.7441; 18, 3178.246, 43.5035;

\section{Calculation summary for 1,3 Migration of $\mathrm{Br}$ TS2 iE (acetonitrile)}

Geometry optimisation and frequency calculation: Program version = DEC-AXP-OSF/1-G03RevB.05. Method and basis set = RB3LYP/6-31G(d). Calculated Energy = -2817.1463277 (hartree), -11781.3059424414 (kJ mol $^{-}$ ${ }^{1}$ ). Higher level single-point calculation: Program = DEC-AXP-OSF/1-G03RevB.05. Method and basis set =

RB3LYP/6-311++G(3df,2p). Calculated Energy = -2819.6885131 (hartree), -11791.9373617842 (kJ mol-1). Structure definition (3D Coordinates: Atom, X coord/Å, Y coord/Å, Z coord/Å): C, -1.056766061, 1.3906834591, -0.0006598138; C, -1.7476215669, 0.2342438935, -0.0008885883; O, -2.5825616329, 0.5715474896, -0.00131421; C, 0.3500876051, 1.4459365123, 0.0003703221; N, 1.338623202, 2.0965649637, 0.0007780709; Br, 0.7371671204, -0.9667770678, 0.000275542; H, -1.6317665139, 2.3222154039, 0.0013380724; H, 1.846848085, 2.9862239523, 0.0038297653. Harmonic Frequencies (Number, Frequency $\left(\mathrm{cm}^{-1}\right)$, IR Intensity $\left.\left(\mathrm{km} \mathrm{mol}^{-1}\right)\right): 1,-429.1544,474.7189 ; 2,85.9371,41.6862 ; 3,87.0143,28.1137 ; 4$, 167.5216, 137.8220; 5, 211.0508, 132.3413; 6, 265.5022, 58.0925; 7, 376.5803, 35.6828; 8, 477.5214, 22.5575; 9, 546.7828, 121.7760; 10, 558.1094, 11.8715; 11, 642.6956, 105.0739; 12, 1005.8027, 290.1731; 13, 1118.0407, 16.1113; 14, 1419.9668, 33.9685; 15, 2114.6678, 2148.9825; 16, 2193.0947, 466.3476; 17, 3076.064, 79.5228; 18, 3323.6678, 1929.9730;

\section{Calculation summary for 1,3 Migration of Br TS2 iZ (acetonitrile)}

Geometry optimisation and frequency calculation: Program version = DEC-AXP-OSF/1-G03RevB.05. Method 
and basis set = RB3LYP/6-31G(d). Calculated Energy = -2817.1546369 (hartree), -11781.3406915158 (kJ mol ${ }^{-}$ 1). Higher level single-point calculation: Program = DEC-AXP-OSF/1-G03RevB.05. Method and basis set = RB3LYP/6-311++G(3df,2p). Calculated Energy = -2819.6951918 (hartree), -11791.9652921076 (kJ mol $\left.{ }^{-1}\right)$. Structure definition (3D Coordinates: Atom, X coord/Å, Y coord/Å, Z coord/Å): C, 1.4747218118, 0.1306642256, -0.9079618445; C, 1.4782120966, 0.0267889784, 0.4845965292; N, 2.0987940585, 0.0079060397, 1.5031793583; C, 0.2950372337, 0.1412155385, -1.583344544; Br, -1.0119309097, 0.0942237292, 0.5907057759; O, -0.3918075148, 0.1881879611, -2.5254237771; H, 2.4130760005, 0.2009118814, -1.4647355252; H, 1.9595806929, -0.1452552246, 2.5114272333. Harmonic Frequencies (Number, Frequency $\left(\mathrm{cm}^{-1}\right)$, IR Intensity $\left(\mathrm{km} \mathrm{mol}^{-1}\right)$ ): 1, -313.8243, 466.7850; 2, 62.3217, 107.0809; 3, 126.8284, 208.3026; 4, 174.7934, 160.6035; 5, 266.6866, 73.2758; 6, 312.3108, 70.6100; 7, 500.7482, 9.1939; 8, 519.9589, 399.4566; 9, 563.6503, 159.6066; 10, 567.5005, 368.3158; 11, 645.5735, 65.0637; 12, 1026.8728 , 328.0744; 13, 1100.8343, 30.6686; 14, 1413.3066, 30.6768; 15, 2071.6613, 2478.6561; 16, 2145.8935, 133.2141; 17, 3091.4222, 82.9818; 18, 3296.3486, 1327.8511;

\section{Calculation summary for $\mathbf{1 , 3}$ Migration of $\mathrm{Br} 1 s E$ iE (acetonitrile)}

Geometry optimisation and frequency calculation: Program version = x86-Linux-G03RevB.01. Method and basis set = RB3LYP/6-31G(d). Calculated Energy = -2817.1653903 (hartree), -11781.3856622346 (kJ mol ${ }^{-1}$ ). Higher level single-point calculation: Program = x86-Linux-G03RevB.01. Method and basis set = RB3LYP/6311++G(3df,2p). Calculated Energy = -2819.705377 (hartree), -11792.007886614 $\left(\mathrm{kJ} \mathrm{mol}^{-1}\right)$. Structure definition (3D Coordinates: Atom, X coord/Å, Y coord/Å, Z coord/Å): C, 1.6397675639, 0.2254610848, 0.1739614438; C, 1.6363203181, -0.0166590833, 1.1341504724; O, 1.6885231193, -0.2217782782, 2.2776703225; C, 0.4469196029, 0.236892934, -0.9971989147; N, 0.3030219558, 0.447754788, 2.2306474654; Br, -1.1940510402, -0.1456615852, 0.0267159894; H, 2.611807806, 0.4245127689, 0.6316582613; H, 1.2125950472, 0.6394158096, -2.6881723718. Harmonic Frequencies (Number, Frequency $\left(\mathrm{cm}^{-1}\right)$, IR Intensity $\left.\left(\mathrm{km} \mathrm{mol}^{-1}\right)\right): 1,104.1121,7.1421 ; 2,117.9864,0.9060 ; 3,311.3467,25.4076 ; 4,335.6371$, 2.0416; 5, 484.6253, 9.6389; 6, 513.7803, 139.5710; 7, 538.7771, 31.3915; 8, 623.1077, 0.6999; 9, 635.1887, 13.5708; 10, 809.4116, 181.8569; 11, 1005.2952, 177.5731; 12, 1128.1984, 63.5949; 13, 1230.6957, 383.6420; 14, 1402.8586, 25.3702; 15, 1707.2188, 783.0043; 16, 2200.312, 1464.5069; 17, 3099.6124, 73.5934; 18, 3215.9877, 159.0430;

\section{Calculation summary for 1,3 Migration of $\operatorname{Br} 1 \mathrm{sE}$ iZ (acetonitrile)}

Geometry optimisation and frequency calculation: Program version = DEC-AXP-OSF/1-G03RevB.05. Method and basis set = RB3LYP/6-31G(d). Calculated Energy = -2817.1692322 (hartree), -11781.4017290604 (kJ mol $^{-}$ 1). Higher level single-point calculation: Program = DEC-AXP-OSF/1-G03RevB.05. Method and basis set =

RB3LYP/6-311++G(3df,2p). Calculated Energy = -2819.7088436 (hartree), -11792.0223839352 (kJ mol-1). Structure definition (3D Coordinates: Atom, X coord/A, Y coord/Å, Z coord/Å): C, -1.2164816322, 1.1651775446, 0.0003121804; C, 0.2275345152, 1.1237396724, 0.0000666781; N, 0.9836235061, 2.1249023564, -0.0002783924; C, -1.9911768317, 0.0839468639, -0.0001769302; Br, 0.929090979, 0.7602762873, 0.0001656113; O, -2.7242171918, -0.818765412, -0.0005852629; H, -1.7203977727, 2.1338216397, 0.0002998395; H, 1.9913301916, 1.9144707318, -0.0006769559. Harmonic Frequencies

(Number, Frequency $\left(\mathrm{cm}^{-1}\right)$, IR Intensity $\left(\mathrm{km} \mathrm{mol}^{-1}\right)$ ): 1, 108.13, 1.5889; 2, 114.9412, 1.0993; 3, 300.4198, 46.9222; 4, 330.1739, 12.7767; 5, 477.8509, 23.7598; 6, 495.2132, 113.4317; 7, 535.8473, 19.1804; 8, 619.6791, 3.7344; 9, 646.4935, 126.6575; 10, 770.7583, 77.8343; 11, 1009.4635, 230.9349; 12, 1113.2829, 142.3756; 13, 1164.0265, 552.0182; 14, 1413.2144, 37.6547; 15, 1748.544, 724.2171; 16, 2204.9128, 1436.4174; 17, 3109.0877, 87.3086; 18, 3300.1703, 253.3347; 


\section{Calculation summary for 1,3 Migration of $\mathrm{Br} 1 \mathrm{sZ}$ iE (acetonitrile)}

Geometry optimisation and frequency calculation: Program version = x86-Linux-G03RevB.01. Method and basis set = RB3LYP/6-31G(d). Calculated Energy = -2817.1607841 (hartree), -11781.3663991062 (kJ mol ${ }^{-1}$ ). Higher level single-point calculation: Program = x86-Linux-G03RevB.01. Method and basis set $=$ RB3LYP/6311++G(3df,2p). Calculated Energy = -2819.7016701 (hartree), -11791.9923843582 (kJ mol $\left.{ }^{-1}\right)$. Structure definition (3D Coordinates: Atom, X coord/Å, Y coord/Å, Z coord/Å): C, 0.857426451, 0.0959681337, 1.0027569085; C, 0.9661013131, 0.1183807316, 2.3283584197; H, 1.7890544026, 0.1875067686, 0.4458524318; O, 1.0738949853, 0.1390922052, 3.4857265574; C, -0.4250601261, -0.0418447489, 0.3243728559; N, -1.6157022649, -0.1620356165, 0.7183456484; Br, -0.1644658064, -0.0296291963, 1.6297903535; H, -1.7048010353, -0.1639979221, 1.7496488372. Harmonic Frequencies (Number, Frequency $\left(\mathrm{cm}^{-1}\right)$, IR Intensity $\left.\left(\mathrm{km} \mathrm{mol}^{-1}\right)\right): 1,44.3856,12.4692 ; 2,126.7789,8.4099 ; 3,258.06,6.4786 ; 4,341.9336$, 18.1581; 5, 499.313, 59.6902; 6, 500.1544, 35.2160; 7, 533.3313, 18.5029; 8, 619.7885, 23.8070; 9, 732.4799, 349.9700; 10, 780.6012, 147.5851; 11, 980.2009, 109.3239; 12, 1147.0724, 18.1996; 13, 1186.1965, 286.3719; 14, 1408.5703, 298.5006; 15, 1702.4987, 404.6661; 16, 2195.9178, 1502.2908; 17, 3134.9602, 95.3779; 18, 3226.7991, 90.5668;

\section{Calculation summary for 1,3 Migration of $\mathrm{Br} 1 \mathrm{sZ}$ iZ (acetonitrile)}

Geometry optimisation and frequency calculation: Program version = x86-Linux-G03RevB.01. Method and basis set = RB3LYP/6-31G(d). Calculated Energy = -2817.1672603 (hartree), -11781.3934825746 (kJ mol ${ }^{-1}$ ). Higher level single-point calculation: Program = x86-Linux-G03RevB.01. Method and basis set = RB3LYP/6311++G(3df,2p). Calculated Energy = -2819.7074806 (hartree), -11792.0166838692 (kJ mol $\left.{ }^{-1}\right)$. Structure definition (3D Coordinates: Atom, X coord/Å, Y coord/Å, Z coord/Å): C, 0.8851295423, 0.0543651911, 1.0219624284; C, 0.9289854047, 0.0283161914, 2.352304911; H, 1.82813034, 0.1472359055, 0.4883953043; O, 0.9794304568, 0.0064132992, 3.5130411286; C, -0.3940992132, -0.0404358474, 0.3423900894; N, 1.5230280879, -0.148449495, 0.8847291198; Br, -0.1486714712, 0.0225680809, -1.6377333785; H, 2.3189702881, -0.2027518774, 0.2348955019. Harmonic Frequencies (Number, Frequency (cm ${ }^{-1}$ ), IR Intensity $\left.\left(\mathrm{km} \mathrm{mol}^{-1}\right)\right):$ 1, 60.3112, 0.6002; 2, 112.1785, 7.9343; 3, 250.2446, 12.4347; 4, 333.2748, 36.0661; 5, 473.4775, 69.1138; 6, 510.063, 13.9522; 7, 530.5133, 21.9323; 8, 627.8501, 72.6911; 9, 752.2235, 139.8362; 10, 754.1549, 245.3471; 11, 1006.6816, 70.9396; 12, 1129.6511, 750.3699; 13, 1141.393, 4.1662; 14, 1409.7496, 326.5109; 15, 1731.3493, 361.8095; 16, 2198.8821, 1540.9253; 17, 3158.5982, 94.8588; 18, 3308.6665, 210.1214;

\section{1,3 Migration of $\mathrm{CH}_{3}$}

\section{Calculation summary for 1,3 Migration of $\mathrm{CH}_{3} 3 \mathrm{sE}$ (vacuum)}

Geometry optimisation and frequency calculation: Program version = x86-Linux-G03RevB.01. Method and basis set = RB3LYP/6-31G(d). Calculated Energy = -285.3625057 (hartree), $-1193.3859988374\left(\mathrm{~kJ} \mathrm{~mol}^{-1}\right)$. Higher level single-point calculation: Program = x86-Linux-G03RevB.01. Method and basis set = RB3LYP/6311+G(3df,2p). Calculated Energy = -285.4736831 (hartree), -1193.8509427242 (kJ mol $\left.{ }^{-1}\right)$. Structure definition (3D Coordinates: Atom, X coord/Å, Y coord/Å, Z coord/Å): C, 0.8198298139, 0.0615260751, 0.4086547745; C, 1.218152772, -0.0520762968, 0.8494016008; N, 1.5640941671, -0.0305127151, 2.0210969588; C, -0.6006977642, 0.0611519411, -0.8308969866; C, -1.6676995028, -0.0695983869, 0.2445557166; O, -0.886368339, 0.1619797074, -2.0132286139; H, 1.5653945187, 0.1511552557, - 
1.1927582321; H, 1.7550430062, -0.9208968393, 2.485559784; H, -2.6516505288, 0.0121330269, 0.2203934314; H, -1.5882262856, -1.0394742629, 0.7509923254; H, -1.5557850812, 0.7088141713, 1.0083164162. Harmonic Frequencies (Number, Frequency ( $\mathrm{cm}^{-1}$ ), IR Intensity $\left(\mathrm{km} \mathrm{mol}^{-1}\right)$ ): 1, 60.7649, 2.1247; 2, 118.7003, 3.3100; 3, 146.4485, 1.6221; 4, 382.8502, 3.3755; 5, 452.2215, 38.1023; 6, 487.968, 24.3463; 7, 498.9578, 13.8575; 8, 599.8864, 16.4400; 9, 658.9797, 35.8734; 10, 805.7001, 1.1898; 11, 868.8258, 62.7446; 12, 972.9898, 402.1273; 13, 1020.4221, 8.1925; 14, 1054.9684, 7.0703; 15, 1132.2003, 19.9730; 16, 1257.3301, 146.5828; 17, 1391.2407, 23.6779; 18, 1413.8309, 36.5803; 19, 1496.3352, 8.5083; 20, 1507.7356, 9.7644; 21, 1775.2772, 333.6819; 22, 2129.1823, 496.2705; 23, 3053.512, 3.6516; 24, 3113.6443, 9.9863; 25, 3170.6626, 8.1577; 26, 3214.3436, 3.3563; 27, 3471.4983, 22.1497;

\section{Calculation summary for 1,3 Migration of $\mathrm{CH}_{3} 3 \mathrm{sZ}$ (vacuum)}

Geometry optimisation and frequency calculation: Program version = x86-Linux-G03RevB.01. Method and basis set $=$ RB3LYP/6-31G(d). Calculated Energy = -285.3611353 (hartree), $-1193.3802678246\left(\mathrm{~kJ} \mathrm{~mol}^{-1}\right)$. Higher level single-point calculation: Program = x86-Linux-G03RevB.01. Method and basis set $=$ RB3LYP/6$311+G(3 d f, 2 p)$. Calculated Energy = -285.4722704 (hartree), $-1193.8450348128\left(\mathrm{~kJ} \mathrm{~mol}^{-1}\right)$. Structure definition (3D Coordinates: Atom, X coord/Å, Y coord/Å, Z coord/Å): C, 0.8094802122, 0.0461817978, 0.015313416; C, 0.8147238429, 0.0683047315, 1.3121244386; H, 1.7598644167, 0.0903112709, 0.538623947; N, 0.8195112102, 0.0885669091, 2.5280336396; C, -0.466179357, -0.0094489457, 0.7664647167; O, -1.5546331042, -0.0614561809, -0.2146628054; C, -0.3293099356, -0.0065971631, 2.2810778431; H, 0.8349605367, -0.7976716274, 3.0345475593; H, -1.3194438928, -0.0811690216, 2.7341417257; H, 0.1598645207, 0.9153128661, -2.6199674637; H, 0.2929522065, -0.8457449279, -

2.6163582336. Harmonic Frequencies (Number, Frequency $\left(\mathrm{cm}^{-1}\right)$, IR Intensity $\left(\mathrm{km} \mathrm{mol}^{-1}\right)$ ): 1, 88.0033, 3.2975; 2, 108.3302, 0.4408; 3, 142.8238, 1.9241; 4, 355.7762, 1.5262; 5, 457.575, 4.2622; 6, 466.9615, 54.8553; 7, 490.47, 32.9265; 8, 635.7577, 30.6133; 9, 640.7193, 25.9119; 10, 844.128, 60.7415; 11, 897.8376, 17.7615; 12, 943.313, 422.3741; 13, 972.8706, 5.2116; 14, 1053.0366, 6.1099; 15, 1152.13, 7.6200; 16, 1190.242, 95.2033; 17, 1401.5015, 15.6340; 18, 1436.2305, 186.3880; 19, 1497.1215, 10.9940; 20, 1504.7893, 8.5758; 21, 1769.9567, 177.3579; 22, 2144.0478, 600.6421; 23, 3048.6429, 4.2629; 24, 3106.7083, 12.0867; 25, 3170.1499, 9.9746; 26, 3206.8018, 1.0737; 27, 3492.8712, 33.9826;

\section{Calculation summary for $\mathbf{1 , 3}$ Migration of $\mathrm{CH}_{3}$ TS2 $\mathrm{iE}$ (vacuum)}

Geometry optimisation and frequency calculation: Program version $=$ x86-Linux-G03RevB.01. Method and basis set $=$ RB3LYP/6-31G(d). Calculated Energy = -285.261512 (hartree), $-1192.963643184\left(\mathrm{~kJ} \mathrm{~mol}^{-1}\right)$. Higher level single-point calculation: Program = x86-Linux-G03RevB.01. Method and basis set $=$ RB3LYP/6-

$311+G(3 d f, 2 p)$. Calculated Energy $=-285.3741434$ (hartree), $-1193.4346676988\left(\mathrm{~kJ} \mathrm{~mol}^{-1}\right)$. Structure definition (3D Coordinates: Atom, X coord/Å, Y coord/Å, Z coord/Å): C, -0.0748593229, 0.8351867644, 0.7761636575; C, -0.2428918413, -0.5331522028, -0.9394215018; N, -0.4275119803, -1.5636962824, 1.5748405265; C, 0.2216094416, 0.9337457031, 0.5651509115; O, 0.5030593545, 1.6416487592, 1.4734862926; C, -0.0114179835, -0.9732080683, 1.0486145013; H, -0.0992435202, 1.6149264728, 1.5329397111; H, -0.2169332602, -1.6330193478, -2.5658067397; H, -0.7705683479, -1.728574267, 0.8271746976; H, 0.9775586747, -1.4336693182, 1.0821190399; H, -0.2773462835, -0.5824128157, 2.036364537. Harmonic Frequencies (Number, Frequency $\left(\mathrm{cm}^{-1}\right)$, IR Intensity $\left(\mathrm{km} \mathrm{mol}^{-1}\right)$ ): $1,-779.3373$, 440.4628; 2, 74.4952, 1.6851; 3, 133.92, 0.9352; 4, 288.4484, 35.2536; 5, 376.9146, 136.3019; 6, 404.8146, 49.8932; 7, 480.0622, 34.4941; 8, 500.1263, 10.8748; 9, 544.0084, 14.5742; 10, 599.356, 16.2403; 11, 723.9039, 76.2565; 12, 795.1273, 147.3879; 13, 854.0254, 55.7355; 14, 882.0075, 4.4091; 15, 1081.7658, 2.3186; 16, 1178.9772, 73.2615; 17, 1280.646, 0.8418; 18, 1378.1618, 1.8671; 19, 1469.8465, 1.6594; 20, 1502.3037, 11.7373; 21, 1983.0255, 1157.1941; 22, 2035.0772, 173.1585; 23, 3058.6521, 1.5638; 24, 
3124.8297, 0.6229; 25, 3184.4988, 10.4964; 26, 3200.15, 8.9727; 27, 3570.1916, 82.1979;

\section{Calculation summary for 1,3 Migration of $\mathrm{CH}_{3}$ TS2 iZ (vacuum)}

Geometry optimisation and frequency calculation: Program version = x86-Linux-G03RevB.01. Method and basis set = RB3LYP/6-31G(d). Calculated Energy = -285.2695504 (hartree), -1192.9972597728 (kJ mol $\left.{ }^{-1}\right)$. Higher level single-point calculation: Program = x86-Linux-G03RevB.01. Method and basis set = RB3LYP/6311+G(3df,2p). Calculated Energy = -285.3818266 (hartree), $-1193.4667988412\left(\mathrm{~kJ} \mathrm{~mol}^{-1}\right)$. Structure definition (3D Coordinates: Atom, X coord/Å, Y coord/Å, Z coord/Å): C, 0.0597711136, -1.1565117727, 0.0094059545; C, -1.0524388864, -0.3282927727, -0.0117369545; N, -2.2702408864, -0.2130067727, 0.0275260455; C, 1.0939531136, -0.2508727727, 0.0057500455; O, 2.2666211136, -0.0741937727, 0.0023780455; C, -0.0073528864, 1.4395762273, 0.0005300455; H, 0.0941511136, -2.2412697727, 0.0188119545; H, -2.8093888864, 0.6306982273, -0.1032939545; H, -0.5730058864, 1.7761542273, 0.8742409545; H, -0.4860688864, 1.7656002273, 0.9279460455; H, 0.9694351136, 1.9300212273, 0.0541289545. Harmonic Frequencies (Number, Frequency $\left(\mathrm{cm}^{-1}\right)$, IR Intensity $\left(\mathrm{km} \mathrm{mol}^{-1}\right)$ ): 1, -728.978, 544.5476; 2, 78.5388, 5.8686; 3, 155.9481, 51.5788; 4, 194.784, 91.2499; 5, 292.2007, 15.8888; 6, 428.7236, 38.2451; 7, 443.3367, 7.3986; 8, 490.9338, 4.2949; 9, 576.3002, 27.4868; 10, 595.8788, 10.8664; 11, 724.3573, 61.1522; 12, 762.3822, 309.7782; 13, 866.134, 5.7353; 14, 903.223, 66.5380; 15, 1075.6015, 18.0951; 16, 1179.8585, 85.6245; 17, 1269.6741, 0.5115; 18, 1379.7874, 0.8296; 19, 1488.1952, 3.9395; 20, 1503.895, 9.0795; 21, 1997.6572, 951.8894; 22, 2036.9701, 227.0049; 23, 3049.9715, 5.8645; 24, 3127.6342, 4.3187; 25, 3157.7503, 14.5768; 26, 3218.9259, 4.1386; 27, 3657.1018, 126.7340;

\section{Calculation summary for 1,3 Migration of $\mathrm{CH}_{3} 1 \mathrm{sE}$ (vacuum)}

Geometry optimisation and frequency calculation: Program version = x86-Linux-G03RevB.01. Method and basis set = RB3LYP/6-31G(d). Calculated Energy = -285.366018 (hartree), -1193.400687276 (kJ mol-1). Higher level single-point calculation: Program = x86-Linux-G03RevB.01. Method and basis set = RB3LYP/6311+G(3df,2p). Calculated Energy = -285.4767754 (hartree), $-1193.8638747228\left(\mathrm{~kJ} \mathrm{~mol}^{-1}\right)$. Structure definition (3D Coordinates: Atom, X coord/Å, Y coord/A, Z coord/Å): C, 0.8834282846, 0.1579702811, 0.1264800447; C, 0.941889272, -0.144150511, 1.1629744618; O, 0.9833982116, -0.4117815442, 2.2990479421; C, -0.3735228067, 0.1639694428, -0.9047869982; N, -0.4444784035, 0.4537051397, 2.1506622222; C, -1.6452013604, -0.1960356161, -0.1715040314; H, 1.8249174226, 0.4094123776, 0.6061237229; H, 0.4798187128, 0.6793981037, -2.5340315991; H, -2.4842435598, -0.1498864687, 0.8674364295; H, -1.5848804232, -1.2076727345, 0.249436882; H, -1.8310093577, 0.4965435179, 0.6591865632. Harmonic Frequencies (Number, Frequency $\left(\mathrm{cm}^{-1}\right)$, IR Intensity $\left(\mathrm{km} \mathrm{mol}^{-1}\right)$ ): 1, 92.1568, 5.1985; 2, 142.3225, 3.5809; 3, 163.3868, 0.0020; 4, 390.5191, 0.9274; 5, 475.7032, 13.8185; 6, 479.9372, 11.8948; 7, 533.3462, 11.4536; 8, 617.3069, 10.6809; 9, 626.4668, 3.5719; 10, 836.0782, 3.2928; 11, 908.8031, 97.4125; 12, 1041.7626, 12.7179; 13, 1086.1237, 1.7156; 14, 1122.3727, 24.6967; 15, 1156.7194, 14.7251; 16, 1368.0563, 205.4494; 17, 1392.2708, 24.9162; 18, 1437.6556, 43.9679; 19, 1503.3352, 7.0730; 20, 1514.9936, 10.5458; 21, 1701.0039, 167.5552; 22, 2219.6223, 767.8544; 23, 3047.4944, 10.1342; 24, 3100.8734, 15.0064; 25, 3170.7814, 5.3854; 26, 3204.6226, 4.2935; 27, 3414.5225, 10.4949;

\section{Calculation summary for 1,3 Migration of $\mathrm{CH}_{3} 1 \mathrm{sE}$ iZ (vacuum)}

Geometry optimisation and frequency calculation: Program version = x86-Linux-G03RevB.01. Method and basis set = RB3LYP/6-31G(d). Calculated Energy = -285.3654898 (hartree), -1193.3984783436 $\left(\mathrm{kJ} \mathrm{mol}^{-1}\right)$. Higher level single-point calculation: Program = x86-Linux-G03RevB.01. Method and basis set $=$ RB3LYP/6- 
311+G(3df,2p). Calculated Energy = -285.4768394 (hartree), -1193.8641423708 $\left(\mathrm{kJ} \mathrm{mol}^{-1}\right)$. Structure definition (3D Coordinates: Atom, X coord/Å, Y coord/A, Z coord/Å): C, 0.8872794191, 0.0093295876, 0.1742767423; C, -0.386012959, 0.0379523173, -0.9109637951; N, -0.3182609547, 0.0925513974, 2.190061079; C, 0.987988001, -0.0466027977, 1.1445694771; C, -1.6609859835, 0.0002494999, 0.0897552346; O, 1.0838722781, -0.0959585595, 2.3087170308; H, 1.8112516695, 0.0355450847, 0.7433742413; H, -1.2614872175, 0.1075695801, -2.5905252801; H, -2.5414091972, 0.0259542028, 0.7380064241; H, -1.7086597368, -0.9107667156, 0.5199707497; H, -1.7124579258, 0.8559348999, 0.5951842719. Harmonic Frequencies (Number, Frequency $\left(\mathrm{cm}^{-1}\right)$, IR Intensity $\left(\mathrm{km} \mathrm{mol}^{-1}\right)$ ): 1, 84.9744, 1.9954; 2, 138.5151, 0.3498; 3, 140.0537, 4.8173; 4, 394.968, 2.0836; 5, 478.1767, 15.4213; 6, 482.6548, 11.2139; 7, 523.6456, 10.0502; 8, 635.4494, 10.2767; 9, 652.6877, 57.4012; 10, 822.7935, 0.4773; 11, 872.1367, 27.3712; 12, 1033.4076, 23.2171; 13, 1078.0004, 13.0737; 14, 1103.9708, 66.8959; 15, 1147.2536, 53.6957; 16, 1362.0215, 21.6347; 17, 1418.7823, 11.1692; 18, 1440.4397, 53.9491; 19, 1506.8938, 9.3658; 20, 1513.1032, 15.7267; 21, 1692.3531, 181.3790; 22, 2220.8338, 769.7512; 23, 3045.1094, 7.2788; 24, 3103.4344, 11.3706; 25, 3138.6684, 15.4776; 26, 3217.9809, 14.0447; 27, 3427.857, 2.6206;

\section{Calculation summary for 1,3 Migration of $\mathrm{CH}_{3} 1 \mathrm{sZ} \mathrm{iE} \mathrm{(vacuum)}$}

Geometry optimisation and frequency calculation: Program version = x86-Linux-G03RevB.01. Method and basis set = RB3LYP/6-31G(d). Calculated Energy = -285.3631751 (hartree), -1193.3887982682 (kJ mol $\left.{ }^{-1}\right)$. Higher level single-point calculation: Program = x86-Linux-G03RevB.01. Method and basis set = RB3LYP/6-

311+G(3df,2p). Calculated Energy = -285.4739159 (hartree), -1193.8519162938 (kJ mol $\left.{ }^{-1}\right)$. Structure definition (3D Coordinates: Atom, X coord/Å, Y coord/A, Z coord/Å): C, 0.7893034488, 0.1190696953, 0.0075310613; C, 0.8720941839, 0.0511076725, 1.31262165; H, 1.7201269375, 0.2912386096, 0.5390459009; O, 0.9174933803, -0.0129779501, 2.4771842123; C, -0.4857591904, -0.0270616844, 0.7538948962; N, -1.655912648, -0.2318543712, -0.2745803904; C, -0.3332421442, 0.0869358452, 2.2537899549; H, -1.6486147418, -0.2929976521, 0.7501631801; H, -1.3104419399, -0.0311022909, 2.7246031965; H, 0.0870589458, 1.0622015005, -2.5301588123; H, 0.3489345035, -0.6828451401, 2.636200662. Harmonic Frequencies (Number, Frequency $\left(\mathrm{cm}^{-1}\right)$, IR Intensity $\left.\left(\mathrm{km} \mathrm{mol}^{-1}\right)\right): 1,54.856,2.4831$; 2, 135.055, 3.0390; 3, 168.3709, 0.1672; 4, 359.7508, 1.2707; 5, 480.1064, 5.3268; 6, 491.8279, 22.0187; 7, 535.9518, 3.4442; 8, 606.603, 18.2718; 9, 637.4797, 24.1280; 10, 887.8835, 6.9450; 11, 893.7145, 81.8391; 12, 992.3053, 21.8678; 13, 1085.9925, 1.3544; 14, 1123.6258, 30.2022; 15, 1148.1286, 1.8852; 16, 1320.3551, 58.4782; 17, 1413.4656, 56.6724; 18, 1442.7606, 150.7471; 19, 1503.6013, 5.2643; 20, 1510.2146, 8.9362; 21, 1699.355, 95.8446; 22, 2218.6476, 714.8509; 23, 3047.3875, 12.4286; 24, 3100.7948, 17.4092; 25, 3171.3539, 6.9609; 26, 3209.9145, 7.2706; 27, 3399.7038, 13.3593;

\section{Calculation summary for 1,3 Migration of $\mathrm{CH}_{3} 1 \mathrm{sZ}$ iZ (vacuum)}

Geometry optimisation and frequency calculation: Program version = x86-Linux-G03RevB.01. Method and basis set = RB3LYP/6-31G(d). Calculated Energy = -285.3654731 (hartree), -1193.3984085042 (kJ mol $\left.{ }^{-1}\right)$. Higher level single-point calculation: Program = x86-Linux-G03RevB.01. Method and basis set $=$ RB3LYP/6-

311+G(3df,2p). Calculated Energy = -285.476671 (hartree), $-1193.863438122\left(\mathrm{~kJ} \mathrm{~mol}^{-1}\right)$. Structure definition (3D Coordinates: Atom, X coord/A, Y coord/Å, Z coord/A): C, 0.8090427979, 0.1156097873, 0.00018878; C, 0.8269317794, 0.053718321, 1.3270612517; H, 1.7585133801, 0.2754424296, -0.4986923681; O, 0.8649361666, 0.0027032097, 2.4895413054; C, -0.4508413595, -0.0281544731, -0.7464519801; N, 1.5443765238, -0.2155757062, -0.1022800245; C, -0.3085716757, 0.0651785108, -2.2529314305; H, 2.331670616, -0.2965691977, -0.7515847464; H, -1.2793175658, -0.0505314245, -2.7437018773; H, 0.1166630157, 1.0336172722, -2.5445182184; H, 0.3675888669, -0.7126676904, -2.6290727878. Harmonic 
Frequencies (Number, Frequency $\left(\mathrm{cm}^{-1}\right)$, IR Intensity $\left.\left(\mathrm{km} \mathrm{mol}^{-1}\right)\right): 1,83.7118,0.9601 ; 2,138.9554,0.0180 ; 3$, 143.5546, 0.2118; 4, 361.8239, 2.7656; 5, 462.5315, 14.1565; 6, 500.3868, 15.3680; 7, 541.3106, 1.2477; 8, 616.9499, 53.4274; 9, 637.6209, 13.6074; 10, 851.901, 42.2968; 11, 922.5916, 0.0594; 12, 983.8403, 24.7266; 13, 1078.9636, 13.7997; 14, 1122.9956, 55.0333; 15, 1148.9735, 2.0232; 16, 1319.2239, 61.9746; 17, 1424.0405, 1.2984; 18, 1471.0526, 151.2446; 19, 1502.8598, 7.5242; 20, 1515.0109, 21.5782; 21, 1693.8134, 104.1692; 22, 2231.8218, 743.5846; 23, 3045.6331, 9.5225; 24, 3104.5405, 13.5133; 25, 3137.7604, 17.6616; 26, 3224.7637, 5.7479; 27, 3442.3626, 2.2610;

\section{Calculation summary for 1,3 Migration of $\mathrm{CH}_{3} 3 \mathrm{sE}$ (acetonitrile)}

Geometry optimisation and frequency calculation: Program version = x86-Linux-G03RevB.01. Method and basis set = RB3LYP/6-31G(d). Calculated Energy = -285.3783548 (hartree), $-1193.4522797736\left(\mathrm{~kJ} \mathrm{~mol}^{-1}\right)$. Higher level single-point calculation: Program = x86-Linux-G03RevB.01. Method and basis set = RB3LYP/6311++G(3df,2p). Calculated Energy = -285.490976 (hartree), -1193.923261632 $\left(\mathrm{kJ} \mathrm{mol}^{-1}\right)$. Structure definition (3D Coordinates: Atom, X coord $/ \AA$, Y coord/Å, Z coord/Å): C, 0.8179847351, 0.0670309657, -0.4160904338; C, 1.2126085585, -0.0518406448, 0.8491144568; N, 1.5549981801, -0.0371776654, 2.0151771233; C, 0.5913711769, 0.0631772339, -0.8258850587; C, -1.6564937675, -0.0697018972, 0.2493797389; O, 0.8931541511, 0.1631959458, -2.0146096521; H, 1.5762214387, 0.1524321374, -1.1937825504; H, 1.7485030346, -0.9313336096, 2.5047740515; H, -2.6442964379, -0.0013063744, -0.2108083128; H, 1.5649588445, -1.0335825239, 0.7649490316; H, -1.5515933383, 0.7164725172, 1.0063929149. Harmonic

Frequencies (Number, Frequency $\left(\mathrm{cm}^{-1}\right)$, IR Intensity $\left(\mathrm{km} \mathrm{mol}^{-1}\right)$ ): 1, 45.7939, 3.0327; 2, 108.4919, 2.1603; 3, 146.5158, 2.3504; 4, 388.3908, 14.8031; 5, 454.1201, 73.0314; 6, 494.0339, 53.0297; 7, 503.1434, 26.5335; 8, 604.6331, 16.6884; 9, 653.1535, 54.3814; 10, 810.4418, 1.7223; 11, 833.252, 105.5922; 12, 882.7499, 833.8372; 13, 1020.2332, 31.1027; 14, 1050.8539, 11.5465; 15, 1130.146, 21.3323; 16, 1263.4934, 229.9281; 17, 1398.1182, 44.6696; 18, 1413.4973, 77.4090; 19, 1488.7695, 26.9844; 20, 1498.7588, 10.7391; 21, 1710.4373, 829.3692; 22, 2120.3187, 954.7971; 23, 3051.339, 1.6589; 24, 3111.6949, 5.9784; 25, 3141.3225, 37.9954; 26, 3161.5331, 24.3247; 27, 3172.5973, 419.6554;

\section{Calculation summary for 1,3 Migration of $\mathrm{CH}_{3} 3 \mathrm{sZ}$ (acetonitrile)}

Geometry optimisation and frequency calculation: Program version = x86-Linux-G03RevB.01. Method and basis set = RB3LYP/6-31G(d). Calculated Energy = -285.3779208 (hartree), -1193.4504647856 $\left(\mathrm{kJ} \mathrm{mol}^{-1}\right)$. Higher level single-point calculation: Program = x86-Linux-G03RevB.01. Method and basis set = RB3LYP/6311++G(3df,2p). Calculated Energy = -285.4903875 (hartree), -1193.920800525 (kJ mol $\left.{ }^{-1}\right)$. Structure definition (3D Coordinates: Atom, X coord/Å, Y coord/Å, Z coord/Å): C, 0.2548426613, -0.7682276459, 0.0000399407; C, 1.5100705237, -0.3223414435, 0.0045331496; H, 0.0873286727, -1.8442846418, 0.0145339075; N, 2.6441167665, 0.0902178878, -0.1166569535; C, -0.8755036054, 0.1698103414, 0.0006498622; O, -0.7309255703, 1.3923832831, -0.0065583706; C, -2.2534295701, -0.466987885, 0.0101436738; H, 3.2140862156, 0.3038690147, 0.723683071; H, -3.0201412664, 0.3105269569, 0.001317275; H, -2.3806744986, -1.1188291356, -0.8631265337; H, -2.377891984, -1.0953938758, 0.9009374496. Harmonic Frequencies (Number, Frequency $\left(\mathrm{cm}^{-1}\right)$, IR Intensity $\left(\mathrm{km} \mathrm{mol}^{-1}\right)$ ): 1, 62.0929, 5.2482; 2, 100.0584, 0.4878; 3, 142.5389, 6.1012; 4, 363.5598, 3.5944; 5, 464.1641, 2.9309; 6, 477.7007, 95.9980; 7, 495.3245, 48.0900; 8, 639.1507, 29.4684; 9, 643.6395, 44.6022; 10, 824.849, 99.4179; 11, 868.3225, 833.4636; 12, 909.8678, 1.7845; 13, 973.5458, 50.4996; 14, 1049.8386, 10.2156; 15, 1151.5336, 12.9725; 16, 1196.0948, 137.3627; 17, 1398.9873, 30.5701; 18, 1438.6359, 373.1569; 19, 1487.5169, 25.3339; 20, 1493.7864, 9.2658; 21, 1710.1595, 358.8735; 22, 2124.3305, 1038.1500; 23, 3047.2917, 1.3046; 24, 3106.3681, 7.0789; 25, 3145.5997, 39.4990; 26, 3161.152, 24.6408; 27, 3172.9704, 412.7427; 
Calculation summary for 1,3 Migration of $\mathrm{CH}_{3}$ TS2 iE (acetonitrile)

Geometry optimisation and frequency calculation: Program version = x86-Linux-G03RevB.01. Method and basis set = RB3LYP/6-31G(d). Calculated Energy = -285.2731792 (hartree), -1193.0124354144 (kJ mol $\left.{ }^{-1}\right)$. Higher level single-point calculation: Program = x86-Linux-G03RevB.01. Method and basis set = RB3LYP/6-

311++G(3df,2p). Calculated Energy = -285.3862751 (hartree), -1193.4854024682 (kJ mol $\left.{ }^{-1}\right)$. Structure definition (3D Coordinates: Atom, X coord/Å, Y coord/Å, Z coord/Å): C, -0.0808320215, 0.8357371129, 0.7781822703; C, -0.2447283044, -0.5417104744, -0.9325408362; N, -0.4154223245, -1.5720023816, 1.5705287144; C, 0.2181841175, 0.927689861, 0.5557636641; O, 0.5206937283, 1.6307959048, 1.4705773784; C, -0.0157674378, -0.9561949892, 1.0536641569; H, -0.09454903, 1.6101815613, 1.5446044296; H, -0.3261947485, -1.5587038504, -2.6003298523; H, -0.7042085103, -1.7682306704, 0.8025912644; H, 0.995223615, -1.35137332, 1.1718358447; H, -0.3890030044, -0.5673533497, 2.0073608588. Harmonic Frequencies (Number, Frequency $\left(\mathrm{cm}^{-1}\right)$, IR Intensity $\left.\left(\mathrm{km} \mathrm{mol}^{-1}\right)\right): 1,-790.1142$, 1151.8112; 2, 65.3034, 1.6877; 3, 119.2948, 3.2306; 4, 287.0958, 38.7291; 5, 332.5947, 200.1452; 6, 380.7213, 80.4723; 7, 478.9359, 48.8588; 8, 499.9903, 22.2570; 9, 542.7228, 27.0176; 10, 602.9842, 24.7010; 11, 716.8512, 115.3713; 12, 776.3238, 211.9598; 13, 842.1986, 105.5442; 14, 880.7227, 5.3788; 15, 1071.0771, 7.6629; 16, 1172.9229, 117.5748; 17, 1275.7059, 1.0642; 18, 1366.927, 10.6549; 19, 1461.2768, 2.3711; 20, 1493.9279, 19.2692; 21, 1923.5096, 2696.6972; 22, 1994.6737, 30.2207; 23, 3053.0362, 0.5894; 24, 3117.1007, 1.2592; 25, 3147.5149, 2.2573; 26, 3177.7229, 12.5894; 27, 3221.9615, 590.9601;

\section{Calculation summary for 1,3 Migration of $\mathrm{CH}_{3}$ TS2 iZ (acetonitrile)}

Geometry optimisation and frequency calculation: Program version = x86-Linux-G03RevB.01. Method and basis set = RB3LYP/6-31G(d). Calculated Energy = -285.2774391 (hartree), -1193.0302503162 (kJ mol $\left.{ }^{-1}\right)$. Higher level single-point calculation: Program = x86-Linux-G03RevB.01. Method and basis set $=$ RB3LYP/6311++G(3df,2p). Calculated Energy = -285.3898192 (hartree), -1193.5002238944 (kJ mol $\left.{ }^{-1}\right)$. Structure definition (3D Coordinates: Atom, X coord/Å, Y coord/Å, Z coord/Å): C, -0.0685648459, -1.1582102312, 0.006222507; C, 1.0424154683, -0.3201940917, -0.0013572027; N, 2.2639162538, -0.2204201059, 0.0105599798; C, -1.0888536728, -0.2428926296, -0.0013324511; C, 0.025364868, 1.4220231075, 0.0026476714; O, -2.2674380278, -0.06045315, 0.0020073817; H, -0.1066777799, -2.2427069686, 0.0064853244; H, 2.7979785701, 0.6400371839, -0.002091418; H, -0.9499443072, 1.9183570105, 0.0366212409; H, 0.5177825788, 1.7534002011, 0.9155802067; H, 0.5707804786, 1.7531215835, 0.891002142. Harmonic Frequencies (Number, Frequency $\left(\mathrm{cm}^{-1}\right)$, IR Intensity $\left(\mathrm{km} \mathrm{mol}^{-1}\right)$ ): 1, -788.8668, 1466.4599; 2, 67.8295, 9.7931; 3, 152.9059, 41.9490; 4, 224.6858, 131.6500; 5, 295.9003, 18.6552; 6, 414.4771, 93.9348; 7, 451.5491, 13.8013; 8, 493.017, 2.7265; 9, 571.3886, 47.2840; 10, 594.8103, 18.4891; 11, 713.4075, 91.2472; 12, 754.1464, 563.8664; 13, 869.6829, 8.2945; 14, 906.5373, 132.5915; 15, 1073.3881, 40.9481; 16, 1174.6994, 143.3232; 17, 1267.2163, 0.1489; 18, 1368.9272, 7.0869; 19, 1472.1461, 8.1904; 20, 1482.9413, 15.9725; 21, 1932.6751, 2448.5574; 22, 1991.4032, 25.7476; 23, 3067.8159, 1.0478; 24, 3136.9032, 3.7973; 25, 3176.0605, 8.8070; 26, 3244.492, 0.2511; 27, 3651.2868, 354.0256;

\section{Calculation summary for 1,3 Migration of $\mathrm{CH}_{3} 1 s E$ iE (acetonitrile)}

Geometry optimisation and frequency calculation: Program version = x86-Linux-G03RevB.01. Method and basis set = RB3LYP/6-31G(d). Calculated Energy = -285.3767888 (hartree), -1193.4457307616 (kJ mol ${ }^{-1}$ ). Higher level single-point calculation: Program = DEC-AXP-OSF/1-G03RevB.05. Method and basis set = RB3LYP/6-311++G(3df,2p). Calculated Energy = -285.4884654 (hartree), -1193.9127623028 (kJ mol $\left.{ }^{-1}\right)$. Structure definition (3D Coordinates: Atom, X coord/Å, Y coord/Å, Z coord/Å): C, 0.8779227716, 
0.1570945282, -0.12838313; C, 0.939484982, -0.1442975667, 1.1601905899; O, 0.9880455103, 0.4110991353, 2.2970943543; C, -0.3765001768, 0.1632205314, -0.9027624201; N, -0.4387688661, 0.4558543626, -2.151806356; C, -1.6483604215, -0.1970521866, -0.1688803818; H, 1.8247310579, 0.4092428054, -0.6089143946; H, 0.4981449688, 0.6808285379, -2.5237799292; H, -2.4934666313, 0.1555137459, -0.8588742741; H, -1.5848418047, -1.2073153055, 0.255212017; H, -1.8328325424, 0.4967784141, 0.66125829. Harmonic Frequencies (Number, Frequency $\left(\mathrm{cm}^{-1}\right)$, IR Intensity $\left(\mathrm{km} \mathrm{mol}^{-1}\right)$ ): 1 , 93.2506, 9.4425; 2, 141.1342, 6.7656; 3, 162.8704, 0.0068; 4, 393.8058, 2.5582; 5, 467.9101, 16.0833; 6, 483.9312, 20.2318; 7, 532.0619, 18.7111; 8, 623.3001, 16.0318; 9, 629.4749, 5.3948; 10, 833.9775, 4.7972; 11, 897.5402, 158.5632; 12, 1037.4895, 26.7015; 13, 1079.8846, 2.1074; 14, 1122.1378, 16.8008; 15, 1159.7982, 36.8927; 16, 1369.0289, 386.4356; 17, 1392.0146, 57.1269; 18, 1434.038, 95.6964; 19, 1497.7755, 15.8259; 20, 1506.0656, 11.1725; 21, 1676.4333, 391.8271; 22, 2181.7616, 1495.1200; 23, 3043.6848, 9.0829; 24, 3098.1533, 12.5802; 25, 3116.3024, 49.3744; 26, 3158.9308, 12.1241; 27, 3269.1213, 20.9084;

\section{Calculation summary for 1,3 Migration of $\mathrm{CH}_{3} 1 \mathrm{sE}$ iZ (acetonitrile)}

Geometry optimisation and frequency calculation: Program version = x86-Linux-G03RevB.01. Method and basis set = RB3LYP/6-31G(d). Calculated Energy = -285.3755499 (hartree), -1193.4405496818 (kJ mol $\left.{ }^{-1}\right)$. Higher level single-point calculation: Program = x86-Linux-G03RevB.01. Method and basis set = RB3LYP/6-

311++G(3df,2p). Calculated Energy = -285.4878369 (hartree), -1193.9101339158 (kJ mol $\left.{ }^{-1}\right)$. Structure definition (3D Coordinates: Atom, X coord/Å, Y coord/Å, Z coord/Å): C, 0.890016974, 0.0091111254, 0.1749055664; C, -0.3825997372, 0.0380343848, -0.9111086587; N, -0.3239441522, 0.0933270132, 2.193591765; C, 0.9848466295, -0.0467733106, 1.1444413718; C, -1.6551122284, 0.0000837986, 0.088204866; O, 1.0783041205, -0.0961716578, 2.3090408981; H, 1.8291541198, 0.0345146252, 0.7281544325; H, -1.2734078335, 0.1080547691, -2.5933861034; H, -2.5362249045, 0.0247619674, 0.7354425417; H, -1.6982667105, -0.9107604101, 0.5224236395; H, -1.7029883973, 0.8567772286, 0.5960409242. Harmonic Frequencies (Number, Frequency $\left(\mathrm{cm}^{-1}\right)$, IR Intensity $\left(\mathrm{km} \mathrm{mol}^{-1}\right)$ ): $1,86.652$, 3.4657; 2, 136.2103, 8.2349; 3, 137.7773, 0.7575; 4, 395.4795, 3.7684; 5, 475.732, 17.5747; 6, 483.4089, 21.2450; 7, 525.4641, 16.6105; 8, 632.8616, 18.4632; 9, 640.0483, 84.8779; 10, 821.5999, 2.4240; 11, 858.0614, 48.5685; 12, 1031.882, 40.2057; 13, 1073.3708, 19.5406; 14, 1102.2533, 107.7269; 15, 1144.7386, 155.8063; 16, 1362.0823, 35.5747; 17, 1415.2218, 17.2092; 18, 1438.9921, 85.0002; 19, 1499.9278, 11.6864; 20, 1504.5952, 35.1306; 21, 1671.2825, 404.6278; 22, 2185.1506, 1482.9998; 23, 3041.3797, 5.3188; 24, 3099.5174, 9.1323; 25, 3129.3095, 57.4715; 26, 3138.5841, 15.4923; 27, 3310.0221, 26.8252;

\section{Calculation summary for $\mathbf{1 , 3}$ Migration of $\mathrm{CH}_{3} 1 \mathrm{sZ} \mathrm{iE}$ (acetonitrile)}

Geometry optimisation and frequency calculation: Program version = x86-Linux-G03RevB.01. Method and basis set = RB3LYP/6-31G(d). Calculated Energy = -285.3737438 (hartree), -1193.4329965716 (kJ mol $\left.{ }^{-1}\right)$. Higher level single-point calculation: Program = x86-Linux-G03RevB.01. Method and basis set = RB3LYP/6-

311++G(3df,2p). Calculated Energy = -285.4853872 (hartree), -1193.8998892704 $\left(\mathrm{kJ} \mathrm{mol}^{-1}\right)$. Structure definition (3D Coordinates: Atom, X coord/Å, Y coord/Å, Z coord/Å): C, 0.292177604, -0.7241290252, 0.0003583285; C, 1.5473927847, -0.3016571263, 0.000195408; H, 0.1659538145, -1.8076031072, 0.0008237416; O, 2.6515015835, 0.0779201673, -0.0000082622; C, -0.8864215539, 0.1692248409, 0.0001585701; N, -0.9009403163, 1.4538704122, -0.000048048; C, -2.2058186787, -0.5701999302, 0.0004240464; H, 0.0503215423, 1.855448318, 0.0005523538; H, -3.0297999822, 0.1461837722, 0.0050639916; H, -2.2859534593, -1.2206598187, -0.8804215398; H, -2.2899333056, -1.2132559445, 0.8846851501. Harmonic Frequencies (Number, Frequency $\left(\mathrm{cm}^{-1}\right)$, IR Intensity $\left(\mathrm{km} \mathrm{mol}^{-1}\right)$ ): 1, 58.0804, 4.3627; 2, 129.683, 4.4604; 3, 165.0974, 0.2656; 4, 365.4828, 2.3304; 5, 484.0071, 6.4292; 6, 496.0332, 34.5881; 7, 534.9799, 7.2179; 8, 608.5723, 26.6077; 9, 638.0265, 30.7806; 10, 873.5474, 138.9455; 11, 
885.8298, 7.1172; 12, 990.4089, 43.7243; 13, 1079.1088, 1.4040; 14, 1132.1255, 42.4911; 15, 1150.0932, 14.7649; 16, 1332.7119, 124.3186; 17, 1411.8747, 80.7278; 18, 1442.4432, 304.4256; 19, 1495.8308, 11.2142; 20, 1499.9603, 9.0385; 21, 1676.3095, 190.7979; 22, 2182.4978, 1292.1449; 23, 3046.0559, 10.3454; 24, 3100.9974, 14.1913; 25, 3121.9483, 54.8709; 26, 3159.5834, 15.0739; 27, 3273.3464, 6.1836;

\section{Calculation summary for 1,3 Migration of $\mathrm{CH}_{3} 1 \mathrm{sZ}$ iZ (acetonitrile)}

Geometry optimisation and frequency calculation: Program version = x86-Linux-G03RevB.01. Method and basis set = RB3LYP/6-31G(d). Calculated Energy = -285.3755741 (hartree), -1193.4406508862 $\left(\mathrm{kJ} \mathrm{mol}^{-1}\right)$. Higher level single-point calculation: Program = x86-Linux-G03RevB.01. Method and basis set = RB3LYP/6-

311++G(3df,2p). Calculated Energy = -285.4875851 (hartree), -1193.9090808882 (kJ mol $\left.{ }^{-1}\right)$. Structure definition (3D Coordinates: Atom, X coord/Å, Y coord/Å, Z coord/Å): C, 0.3000205508, -0.7628655923, 0.000526788; C, 1.5387333568, -0.2907529269, -0.0010706883; H, 0.1875191977, -1.846217895, 0.0012897939; O, 2.63050336, 0.1217798992, -0.0016016265; C, -0.8588141252, 0.1460669025, 0.0006954691; N, -0.6711730579, 1.4182087042, 0.0020920142; C, -2.20256356, -0.5516264045, 0.0002937725; H, -1.5731710189, 1.9150166793, 0.0023019609; H, -3.0189734692, 0.1763750115, 0.000992015; H, -2.3020953325, -1.1968140131, -0.8814641512; H, -2.3033521863, -1.1949917783, 0.8832623192. Harmonic Frequencies (Number, Frequency $\left(\mathrm{cm}^{-1}\right)$, IR Intensity $\left(\mathrm{km} \mathrm{mol}^{-1}\right)$ ): 1, 77.0424, 1.5350; 2, 127.1127, 0.5320; 3, 138.1663, 0.0787; 4, 363.4529, 4.5199; 5, 464.262, 19.7401; 6, 505.4426, 22.3677; 7, 539.7956, 3.2259; 8, 622.2665, 84.0597; 9, 639.2961, 23.2248; 10, 839.782, 69.7309; 11, 916.2555, 0.7525; 12, 985.225, 41.5494; 13, 1074.7718, 20.6178; 14, 1123.0208, 112.6036; 15, 1142.2233, 5.8172; 16 , 1324.698, 119.3926; 17, 1418.8334, 5.9126; 18, 1463.9012, 233.0613; 19, 1493.8628, 9.2292; 20, 1504.4418, 46.5869; 21, 1672.8234, 190.4039; 22, 2185.8169, 1320.6436; 23, 3043.9376, 6.1451; 24, 3103.2415, 10.3545; 25, 3137.8772, 22.8084; 26, 3142.714, 47.9022; 27, 3317.4292, 21.1049;

\section{1,3 Migration of $\mathrm{Cl}$}

\section{Calculation summary for 1,3 Migration of $\mathrm{Cl} 3 \mathrm{sE}$ (vacuum)}

Geometry optimisation and frequency calculation: Program version = x86-Linux-G03RevB.01. Method and basis set = RB3LYP/6-31G(d). Calculated Energy = -705.650651 (hartree), -2951.031022482 (kJ mol $\left.{ }^{-1}\right)$. Higher level single-point calculation: Program = x86-Linux-G03RevB.01. Method and basis set = RB3LYP/6311+G(3df,2p). Calculated Energy = -705.780804 (hartree), -2951.575322328 $\left(\mathrm{kJ} \mathrm{mol}^{-1}\right)$. Structure definition (3D Coordinates: Atom, X coord/Å, Y coord/Å, Z coord/A): C, 1.1035504904, 0.1245472656, -0.5095353846; C, 1.4716524877, -0.0032425469, 0.7632183703; N, 1.8433315538, 0.0125532402, 1.91631107; C, 0.2732921452, 0.0993089255, -0.969294067; Cl, -1.4972019573, -0.1475926063, 0.3833133962; O, 0.647204061, 0.2138904361, -2.0973281156; H, 1.8678107937, 0.2409733876, -1.2718016576; H, 2.0474690938, -0.8545771158, 2.4135878444. Harmonic Frequencies (Number, Frequency ( $\mathrm{cm}^{-1}$ ), IR Intensity $\left.\left(\mathrm{km} \mathrm{mol}^{-1}\right)\right):$ 1, 111.4539, 3.7235; 2, 137.0395, 0.2374; 3, 342.6191, 1.8405; 4, 409.2654, 11.0090; 5, 458.9204, 45.0388; 6, 507.4187, 28.4624; 7, 576.089, 110.5701; 8, 615.0768, 23.9965; 9, 711.8343, 47.6573; 10, 836.68, 73.5361; 11, 904.0094, 441.5677; 12, 1085.703, 174.7875; 13, 1181.8149, 84.8157; 14, 1415.0016, 19.1577; 15, 1871.9726, 518.3598; 16, 2158.0267, 496.5696; 17, 3216.0551, 6.1980; 18, 3512.2142, 60.3650;

\section{Calculation summary for 1,3 Migration of Cl 3 sZ (vacuum)}

Geometry optimisation and frequency calculation: Program version = x86-Linux-G03RevB.01. Method and basis set = RB3LYP/6-31G(d). Calculated Energy = -705.6494999 (hartree), -2951.0262085818 $\left(\mathrm{kJ} \mathrm{mol}^{-1}\right)$. Higher level single-point calculation: Program = x86-Linux-G03RevB.01. Method and basis set = RB3LYP/6- 
311+G(3df,2p). Calculated Energy = -705.7793637 (hartree), -2951.5692989934 (kJ mol $\left.{ }^{-1}\right)$. Structure definition (3D Coordinates: Atom, X coord/A, Y coord/Å, Z coord/Å): C, 0.8279131822, 0.0697203862, 0.3921511876; C, 0.8374845678, 0.1020783042, 1.7244553638; H, 1.7590275594, 0.118941627, 0.1578389086; N, 0.8472429731, 0.1315094098, 2.9345985388; C, -0.448629923, -0.0039173743, 0.3040027425; O, -1.5498033428, -0.0425598865, 0.1599058762; Cl, -0.2043336656, -0.0573014957, 2.1185495553; H, 0.8817637241, -0.732190873, 3.4761217141. Harmonic Frequencies (Number, Frequency $\left(\mathrm{cm}^{-1}\right)$, IR Intensity $\left.\left(\mathrm{km} \mathrm{mol}^{-1}\right)\right): 1$, 84.7679, 3.6118; 2, 137.918, 1.7462; 3, 312.7372, 5.3130; 4, 425.4786, 19.5373; 5, 474.4866, 84.8242; 6, 484.2383, 16.3088; 7, 509.1405, 43.7747; 8, 697.3903, 39.5124; 9, 819.1802, 349.8843; 10, 837.8029, 65.9373; 11, 898.4032, 404.4503; 12, 1028.4387, 166.4252; 13, 1155.8766, 3.8560; 14, 1417.3386, 152.4371; 15, 1858.157, 277.4760; 16, 2157.1841, 645.5200; 17, 3250.1755, 10.5083; 18, 3514.1724, 66.3749;

\section{Calculation summary for 1,3 Migration of Cl TS2 iE (vacuum)}

Geometry optimisation and frequency calculation: Program version = x86-Linux-G03RevB.01. Method and basis set = RB3LYP/6-31G(d). Calculated Energy = -705.6137833 (hartree), -2950.8768417606 (kJ mol $\left.{ }^{-1}\right)$. Higher level single-point calculation: Program = x86-Linux-G03RevB.01. Method and basis set = RB3LYP/6311+G(3df,2p). Calculated Energy = -705.7448146 (hartree), -2951.4248146572 ( $\left.\mathrm{kJ} \mathrm{mol}^{-1}\right)$. Structure definition (3D Coordinates: Atom, X coord/A, Y coord/A, Z coord/Å): C, 0.9489200727, 0.0257660249, 0.8351205178; C, 1.2190282011, 0.0642593459, 0.5101563104; O, 1.9410867028, 0.1235501899, 1.4239393236; C, -0.3937797375, -0.0716876416, -1.1943619861; N, -1.2826669754, -0.1284605566, 2.0047619402; Cl, -1.0338369103, 0.0148960889, 0.9554929335; H, 1.7454492313, 0.1001991461, 1.5698174539; H, -1.3652577685, -0.5526366556, -2.9157862607. Harmonic Frequencies (Number, Frequency $\left(\mathrm{cm}^{-1}\right)$, IR Intensity $\left.\left(\mathrm{km} \mathrm{mol}^{-1}\right)\right):$, -453.9351, 135.2065; 2, 137.2839, 4.1973; 3, 198.249, 36.0596; 4, 322.3804, 52.1109; 5, 373.1792, 198.2204; 6, 397.8106, 123.8261; 7, 484.8868, 67.1041; 8, 541.9482, 5.7782; 9, 568.2893, 52.8450; 10, 614.2838, 70.9026; 11, 682.0294, 127.9707; 12, 1094.6052, 137.6535; 13, 1105.6639, 18.1082; 14, 1398.3855, 7.3171; 15, 2064.2638, 1252.7032; 16, 2144.9451, 274.5447; 17, 3208.2157, 1.0252; 18, 3681.5935, 255.9909;

\section{Calculation summary for 1,3 Migration of Cl TS2 iZ (vacuum)}

Geometry optimisation and frequency calculation: Program version = x86-Linux-G03RevB.01. Method and basis set = RB3LYP/6-31G(d). Calculated Energy = -705.624374 (hartree), -2950.921132068 $\left(\mathrm{kJ} \mathrm{mol}^{-1}\right)$. Higher level single-point calculation: Program = x86-Linux-G03RevB.01. Method and basis set $=$ RB3LYP/6311+G(3df,2p). Calculated Energy = -705.7541685 (hartree), -2951.463932667 (kJ mol $\left.{ }^{-1}\right)$. Structure definition (3D Coordinates: Atom, X coord/Å, Y coord/A, Z coord/Å): C, 1.0910258184, 0.0875655616, -0.6759143437; C, 1.0779865463, 0.0088974121, 0.7084664087; N, 1.673599717, 0.0549815443, 1.7570993552; C, 0.1440310732, 0.0903698556, -1.2827924926; Cl, -1.2268569572, -0.1383128739, 0.6894821299; O, 0.8071165463, 0.1271403173, -2.2431635902; H, 2.0113559502, 0.1128579092, -1.2506045445; H, 1.4370589234, -0.284529378, 2.6764641369. Harmonic Frequencies (Number, Frequency ( $\mathrm{cm}^{-1}$ ), IR Intensity $\left.\left(\mathrm{km} \mathrm{mol}^{-1}\right)\right):$ 1, -382.2657, 116.5639; 2, 135.6826, 2.3952; 3, 237.1122, 52.9637; 4, 306.4372, 147.2505; 5, 367.8136, 71.1436; 6, 417.4721, 101.4595; 7, 527.8527, 9.6362; 8, 560.5277, 54.6413; 9, 580.0753, 8.5127; 10, 657.2332, 63.4959; 11, 730.9483, 427.1838; 12, 1089.7524, 97.5746; 13, 1104.984, 84.7068; 14, 1400.92, 10.0865; 15, 2065.3418, 1176.9997; 16, 2133.1522, 214.8818; 17, 3219.9807, 3.8244; 18, 3694.1788, 260.0353;

\section{Calculation summary for $\mathbf{1 , 3}$ Migration of $\mathrm{Cl} 1 \mathrm{sE}$ iE (vacuum)}


Geometry optimisation and frequency calculation: Program version = x86-Linux-G03RevB.01. Method and basis set = RB3LYP/6-31G(d). Calculated Energy = -705.6430985 (hartree), -2950.999437927 (kJ mol $\left.{ }^{-1}\right)$. Higher level single-point calculation: Program = x86-Linux-G03RevB.01. Method and basis set $=$ RB3LYP/6311+G(3df,2p). Calculated Energy = -705.7741246 (hartree), -2951.5473890772 (kJ mol $\left.{ }^{-1}\right)$. Structure definition (3D Coordinates: Atom, X coord/A, Y coord/A, Z coord/Å): C, 1.183876815, 0.1572027502, 0.1386441492; C, 1.1829986618, -0.0815162123, 1.1705431338; O, 1.2255656069, -0.2852142699, 2.3140175646; C, -0.0243920108, 0.1713483489, -0.9551517389; N, -0.1544850392, 0.3823269586, 2.1928845126; Cl, -1.5104124964, -0.1640709057, -0.0228077223; H, 2.148164844, 0.3486957618, 0.5998612854; H, 0.7508172182, 0.5637257644, -2.6348398385. Harmonic Frequencies (Number, Frequency $\left(\mathrm{cm}^{-1}\right)$, IR Intensity $\left.\left(\mathrm{km} \mathrm{mol}^{-1}\right)\right): 1,104.5473,3.5381 ; 2,132.1649,1.5179 ; 3,357.5921,3.7064 ; 4,429.0859$, 1.7991; 5, 494.693, 3.7502; 6, 539.2189, 21.6965; 7, 604.5328, 65.5171; 8, 639.3089, 8.9222; 9, 642.3655, 11.4260; 10, 834.9479, 104.4276; 11, 1015.3458, 101.4934; 12, 1145.2421, 20.5812; 13, 1264.6098, 219.9742; 14, 1404.9735, 13.9053; 15, 1720.4765, 308.6585; 16, 2240.3698, 761.4647; 17, 3209.0823, 6.4446; 18, 3455.6752, 2.6469;

\section{Calculation summary for 1,3 Migration of Cl 1 sE iZ (vacuum)}

Geometry optimisation and frequency calculation: Program version = x86-Linux-G03RevB.01. Method and basis set = RB3LYP/6-31G(d). Calculated Energy = -705.6491281 (hartree), -2951.0246537142 (kJ mol $\left.{ }^{-1}\right)$. Higher level single-point calculation: Program = x86-Linux-G03RevB.01. Method and basis set $=$ RB3LYP/6311+G(3df,2p). Calculated Energy = -705.7794456 (hartree), -2951.5696414992 (kJ mol $\left.{ }^{-1}\right)$. Structure definition (3D Coordinates: Atom, X coord/A, Y coord/Å, Z coord/Å): C, 1.199281258, -0.0395875513, 0.2033698994; C, -0.0288649742, -0.0157178739, -0.9744213975; N, -0.058960729, -0.0370636085, 2.2321396283; C, 1.2448934229, -0.0184188683, 1.1250512593; Cl, -1.5255361083, 0.0473748943, 0.0606257987; O, 1.3392973005, -0.0013197955, 2.2837579321; H, 2.1420527993, -0.0781924402, 0.7395923237; H, -1.0014504988, -0.0148313789, -2.6196920872. Harmonic Frequencies (Number, Frequency $\left(\mathrm{cm}^{-1}\right)$, IR Intensity $\left.\left(\mathrm{km} \mathrm{mol}^{-1}\right)\right): 1,111.4109,0.8684 ; 2,128.2753,0.7674 ; 3,357.9344,7.0922 ; 4,406.8626$, 21.4661; 5, 494.4575, 14.8306; 6, 532.3383, 19.6180; 7, 569.4144, 71.1389; 8, 638.3047, 3.4085; 9, 667.1703, 60.3889; 10, 819.0804, 63.2211; 11, 1025.5814, 121.0163; 12, 1133.4081, 15.8328; 13, 1226.2317, 246.1306; 14, 1415.4902, 21.2970; 15, 1753.8293, 313.8545; 16, 2243.6077, 763.6654; 17, 3222.1819, 16.7221; 18, 3522.5841, 26.0959;

\section{Calculation summary for 1,3 Migration of Cl $1 \mathrm{sZ}$ iE (vacuum)}

Geometry optimisation and frequency calculation: Program version = x86-Linux-G03RevB.01. Method and basis set = RB3LYP/6-31G(d). Calculated Energy = -705.6394604 (hartree), -2950.9842233928 (kJ mol $\left.{ }^{-1}\right)$. Higher level single-point calculation: Program = x86-Linux-G03RevB.01. Method and basis set = RB3LYP/6$311+G(3 d f, 2 p)$. Calculated Energy = -705.770378 (hartree), -2951.531720796 $\left(\mathrm{kJ} \mathrm{mol}^{-1}\right)$. Structure definition (3D Coordinates: Atom, X coord/A, Y coord/A, Z coord/ $\AA$ ): C, 0.8267892206, 0.0891886893, 0.3778258656; C, 0.9138446687, 0.1190025013, 1.7024295862; H, 1.7514681277, 0.1749200051, -0.1806569844; O, 0.9781615693, 0.14363398, 2.864742357; C, -0.4625593578, -0.051981809, -0.3110521748; N, 1.6453146065, -0.1647459027, 0.118158503; Cl, -0.2376688296, -0.0561391732, -2.0777052252; H, 1.6876355232, -0.153660872, 1.1413777734. Harmonic Frequencies (Number, Frequency ( $\mathrm{cm}^{-1}$ ), IR Intensity $\left(\mathrm{km} \mathrm{mol}^{-1}\right)$ ): 1, 41.5428, 8.1738; 2, 131.5945, 5.6556; 3, 319.0031, 0.9318; 4, 450.3116, 0.2000; 5, 507.2572, 17.6695; 6, 535.9511, 12.8779; 7, 540.5545, 16.0102; 8, 640.2686, 22.1380; 9, 783.5003, 177.9346; 10, 818.2097, 80.2164; 11, 980.6982, 72.4846; 12, 1148.7517, 2.7135; 13, 1207.5755, 133.1650; 14, 1416.6644, 151.2151; 15, 1707.7536, 191.0430; 16, 2227.6858, 740.8111; 17, 3235.5528, 19.7205; 18, 3443.8958, 2.8913; 


\section{Calculation summary for 1,3 Migration of Cl 1 sZ iZ (vacuum)}

Geometry optimisation and frequency calculation: Program version = x86-Linux-G03RevB.01. Method and basis set = RB3LYP/6-31G(d). Calculated Energy = -705.6484754 (hartree), -2951.0219241228 $\left(\mathrm{kJ} \mathrm{mol}^{-1}\right)$. Higher level single-point calculation: Program = x86-Linux-G03RevB.01. Method and basis set $=$ RB3LYP/6311+G(3df,2p). Calculated Energy = -705.778443 (hartree), -2951.565448626 $\left(\mathrm{kJ} \mathrm{mol}^{-1}\right)$. Structure definition (3D Coordinates: Atom, X coord/Å, Y coord/Å, Z coord/Å): C, 0.8453473377, 0.0639053901, 0.3990482319; C, 0.8728329398, 0.0396630278, 1.7300242262; H, 1.7851210363, 0.1541648842, -0.1298180657; O, 0.9231268364, 0.0206442626, 2.8901117711; C, -0.4304462276, -0.0302621782, -0.2949683571; N, 1.5508418708, -0.1356967995, 0.2736586611; Cl, -0.217827335, 0.0223833788, -2.090580411; H, 2.3375822354, -0.1897962658, -0.3714443482. Harmonic Frequencies (Number, Frequency $\left(\mathrm{cm}^{-1}\right)$, IR Intensity ( $\left.\mathrm{km} \mathrm{mol}^{-1}\right)$ ): 1, 71.2516, 0.2274; 2, 138.4331, 1.0007; 3, 312.6966, 1.8410; 4, 423.4878, 28.1504; 5, 507.9301, 31.7207; 6, 513.6367, 13.2446; 7, 538.3257, 16.9544; 8, 642.7024, 36.7527; 9, 798.2538, 128.3913; 10, 801.3108, 97.3279; 11, 1017.391, 49.8037; 12, 1147.4921, 1.6326; 13, 1188.038, 274.4570; 14, 1428.4227, 168.5457; 15, 1736.6959, 199.2848; 16, 2240.7458, 775.3765; 17, 3254.4945, 19.2043; 18, 3528.3968, 22.6130;

\section{Calculation summary for 1,3 Migration of $\mathrm{Cl} 3 \mathrm{sE}$ (acetonitrile)}

Geometry optimisation and frequency calculation: Program version = x86-Linux-G03RevB.01. Method and basis set = RB3LYP/6-31G(d). Calculated Energy = -705.6675973 (hartree), -2951.1018919086 $\left(\mathrm{kJ} \mathrm{mol}^{-1}\right)$. Higher level single-point calculation: Program = x86-Linux-G03RevB.01. Method and basis set = RB3LYP/6311++G(3df,2p). Calculated Energy = -705.7978917 (hartree), -2951.6467830894 (kJ mol $\left.{ }^{-1}\right)$. Structure definition (3D Coordinates: Atom, X coord/Å, Y coord/Å, Z coord/Å): C, 1.1075630707, 0.1281954604, 0.5180792703; C, 1.4708734241, -0.0094446162, 0.7634506158; N, 1.8364367607, -0.0001376818, 1.9095334768; C, -0.2503942574, 0.0988342163, -0.9776994023; Cl, -1.5014120067, -0.1381661617, 0.39127257; O, -0.6573009709, 0.2060334459, -2.0995650713; H, 1.8841104451, 0.2529994488, 1.2736397707; H, 2.074990687, -0.8569888583, 2.4457606543. Harmonic Frequencies (Number, Frequency

$\left(\mathrm{cm}^{-1}\right)$, IR Intensity $\left.\left(\mathrm{km} \mathrm{mol}^{-1}\right)\right): 1,99.8042,4.3982 ; 2,134.909,1.4123 ; 3,339.7201,17.5590 ; 4,387.0586$, 59.5051; 5, 457.9662, 102.3556; 6, 507.3019, 81.6466; 7, 539.2141, 266.9053; 8, 610.1989, 19.1043; 9, 698.0078, 73.7646; 10, 780.1287, 879.2013; 11, 794.6813, 121.5162; 12, 1073.4032, 272.6216; 13, 1170.6796, 116.8563; 14, 1419.2025, 59.8937; 15, 1818.5837, 1408.1640; 16, 2157.5478, 875.1836; 17, 3125.9676, 94.0544; 18, 3153.4549, 648.7628;

\section{Calculation summary for 1,3 Migration of $\mathrm{Cl} 3$ sZ (acetonitrile)}

Geometry optimisation and frequency calculation: Program version = x86-Linux-G03RevB.01. Method and basis set = RB3LYP/6-31G(d). Calculated Energy = -705.6673145 (hartree), -2951.100709239 $\left(\mathrm{kJ} \mathrm{mol}^{-1}\right)$. Higher level single-point calculation: Program = x86-Linux-G03RevB.01. Method and basis set = RB3LYP/6311++G(3df,2p). Calculated Energy = -705.79722 (hartree), -2951.64397404 (kJ mol $\left.{ }^{-1}\right)$. Structure definition (3D Coordinates: Atom, X coord/Å, Y coord/A, Z coord/Å): C, 0.8257555617, 0.0419789095, 0.3926648737; C, 0.8404522722, 0.016933417, 1.7342400941; H, 1.7629133366, 0.0913187922, -0.1551929241; N, 0.8515006798, 0.1242909611, 2.9317121756; C, -0.4400799417, -0.0214583965, -0.283286896; O, 1.5561633527, -0.0869327533, 0.1514789475; Cl, -0.2078089953, 0.0111725387, -2.1309965389; H, 0.901874293, -0.6805502322, 3.586608846. Harmonic Frequencies (Number, Frequency $\left(\mathrm{cm}^{-1}\right)$, IR Intensity $\left.\left(\mathrm{km} \mathrm{mol}^{-1}\right)\right):$ 1, 58.3626, 2.9339; 2, 132.8862, 6.9929; 3, 306.6989, 46.3621; 4, 400.0968, 130.6983; 5, 
472.923, 101.7477; 6, 479.8188, 139.6494; 7, 513.6117, 72.1013; 8, 683.05, 81.2723; 9, 761.3443, 1409.2302; 10, 787.2824, 38.2874; 11, 800.2758, 95.9267; 12, 1036.3223, 244.3882; 13, 1153.8031, 22.1857; 14, 1411.2179, 372.2652; 15, 1811.2127, 628.6222; 16, 2152.7912, 1140.4325; 17, 3148.105, 753.5703; 18, 3171.0156, 41.9282;

\section{Calculation summary for 1,3 Migration of Cl TS2 iE (acetonitrile)}

Geometry optimisation and frequency calculation: Program version = DEC-AXP-OSF/1-G03RevB.05. Method and basis set = RB3LYP/6-31G(d). Calculated Energy = -705.6359339 (hartree), -2950.9694755698 (kJ mol ${ }^{-1}$ ). Higher level single-point calculation: Program = DEC-AXP-OSF/1-G03RevB.05. Method and basis set = RB3LYP/6-311++G(3df,2p). Calculated Energy = -705.7660736 (hartree), -2951.5137197952 (kJ mol ${ }^{-1}$ ). Structure definition (3D Coordinates: Atom, X coord/A, Y coord/Å, Z coord/Å): C, 0.2311992805, 1.28764638, -0.010938013; C, 1.3932518934, -0.6162626905, -0.0309902532; O, 2.4841172711, 0.2297778293, -0.0509517965; C, -1.0290546036, -0.6568454689, 0.0181346467; N, -2.2070053231, 0.7544155295, 0.0419830602; Cl, -0.3059680924, 1.5390355002, 0.0126293162; H, 0.2800464423, 2.3809766893, -0.0173583476; H, -3.0748692004, -1.2989682361, 0.0591546399. Harmonic Frequencies (Number, Frequency ( $\mathrm{cm}^{-1}$ ), IR Intensity $\left(\mathrm{km} \mathrm{mol}^{-1}\right)$ ): 1, -427.462, 400.9352; 2, 89.9859, 24.5264; 3, 128.968, 5.1354; 4, 204.8822, 154.7505; 5, 256.8531, 188.2925; 6, 272.9176, 65.7130; 7, 403.0584, 60.7274; 8, 475.352, 28.3375; 9, 551.2431, 16.8666; 10, 568.5375, 72.7391; 11, 630.7264, 98.6198; 12, 1007.59, 205.7840; 13, 1127.1607, 12.5037; 14, 1422.5886, 45.2467; 15, 2127.9236, 1850.9276; 16, 2218.8818, 647.1649; 17, 3078.0336, 98.7112; 18, 3324.2686, 1805.7972;

\section{Calculation summary for 1,3 Migration of Cl TS2 iZ (acetonitrile)}

Geometry optimisation and frequency calculation: Program version = x86-Linux-G03RevB.01. Method and basis set = RB3LYP/6-31G(d). Calculated Energy = -705.6435077 (hartree), -2951.0011492014 (kJ mol $\left.{ }^{-1}\right)$. Higher level single-point calculation: Program = x86-Linux-G03RevB.01. Method and basis set = RB3LYP/6311++G(3df,2p). Calculated Energy = -705.7728317 (hartree), -2951.5419821694 (kJ mol $\left.{ }^{-1}\right)$. Structure definition (3D Coordinates: Atom, X coord/Å, Y coord/Å, Z coord/Å): C, 1.1276317355, 0.1012475199, 0.7109417484; C, 1.1115904687, -0.0022585985, 0.6803584673; N, 1.6779485947, -0.0365802745, 1.7199615903; C, -0.0161517787, 0.1154972244, -1.429599767; Cl, -1.3267929315, -0.1324773152, 0.8049045368; O, -0.7734098171, 0.1541544774, -2.3061072924; H, 2.0801652275, 0.168128237, 1.2456606709; H, 1.5785304299, -0.1801046516, 2.7325090424. Harmonic Frequencies (Number, Frequency $\left(\mathrm{cm}^{-1}\right)$, IR Intensity $\left.\left(\mathrm{km} \mathrm{mol}^{-1}\right)\right):$ 1, -283.5584, 479.5006; 2, 58.2781, 76.6640; 3, 137.7539, 239.7099; 4, 183.6756, 205.4035; 5, 254.273, 42.6593; 6, 324.6434, 27.3894; 7, 482.0853, 24.5728; 8, 510.7676, 547.9397; 9, 548.422, 35.0760; 10, 554.0246, 48.6938; 11, 618.8426, 59.5247; 12, 1014.2174, 202.8759; 13, 1113.3551, 10.0176; 14, 1423.8428, 50.5331; 15, 2132.7711, 2234.9080; 16, 2207.3817, 211.3315; 17, 3077.173, 118.1399; 18, 3288.7196, 1467.3487;

\section{Calculation summary for 1,3 Migration of Cl $1 \mathrm{sE}$ iE (acetonitrile)}

Geometry optimisation and frequency calculation: Program version = x86-Linux-G03RevB.01. Method and basis set = RB3LYP/6-31G(d). Calculated Energy = -705.65677 (hartree), $-2951.05661214\left(\mathrm{~kJ} \mathrm{~mol}^{-1}\right)$. Higher level single-point calculation: Program = x86-Linux-G03RevB.01. Method and basis set = RB3LYP/6-

311++G(3df,2p). Calculated Energy = -705.7876817 (hartree), -2951.6040848694 (kJ mol $\left.{ }^{-1}\right)$. Structure definition (3D Coordinates: Atom, X coord/Å, Y coord/Å, Z coord/Å): C, 1.1866214545, 0.1578818447, 0.1400173429; C, 1.1891085498, -0.0803475621, 1.1679661617; O, 1.2265914066, -0.2850031136, 2.3121130236; C, -0.0142875039, 0.1724947838, -0.9552892562; N, -0.1533414959, 0.3821155943, - 
2.191907044; Cl, -1.5190067039, -0.165379659, -0.0201447695; H, 2.1550638195, 0.3507630602, 0.60609069; H, 0.7600543633, 0.5657324946, -2.6409604854. Harmonic Frequencies (Number, Frequency $\left(\mathrm{cm}^{-1}\right)$, IR Intensity $\left(\mathrm{km} \mathrm{mol}^{-1}\right)$ ): 1, 103.5566, 6.2716; 2, 128.2077, 2.4530; 3, 356.8035, 8.9992; 4, 414.2845, 1.2642; 5, 495.795, 3.7359; 6, 535.4495, 36.3884; 7, 572.0165, 169.4776; 8, 639.6567, 1.6756; 9, 644.459, 16.8357; 10, 816.4288, 170.6435; 11, 1015.6026, 143.8538; 12, 1132.6857, 48.4778; 13, 1249.0127, 401.6500; 14, 1404.6893, 29.8017; 15, 1702.0289, 745.2813; 16, 2203.0956, 1489.7182; 17, 3108.15, 76.5921; 18 , 3243.3026, 148.6812;

\section{Calculation summary for 1,3 Migration of Cl 1 sE iZ (acetonitrile)}

Geometry optimisation and frequency calculation: Program version = x86-Linux-G03RevB.01. Method and basis set $=$ RB3LYP/6-31G(d). Calculated Energy = -705.6593489 (hartree), $-2951.0673970998\left(\mathrm{~kJ} \mathrm{~mol}^{-1}\right)$. Higher level single-point calculation: Program = x86-Linux-G03RevB.01. Method and basis set $=$ RB3LYP/6$311++G(3 d f, 2 p)$. Calculated Energy = -705.7899023 (hartree), $-2951.6133714186\left(\mathrm{~kJ} \mathrm{~mol}^{-1}\right)$. Structure definition (3D Coordinates: Atom, X coord/Å, Y coord/Å, Z coord/Å): C, 1.2057122967, -0.0402515261, 0.2063964721; C, -0.0170489249, -0.0164363621, -0.9806758802; N, -0.0668479631, -0.0366943238, 2.2369272923; C, 1.2443069243, -0.0184571025, 1.1218545704; Cl, -1.5225646054, 0.0475464218, 0.0676079304; O, 1.3264247135, -0.0009550592, 2.2818917588; H, 2.1622793991, -0.0784151508, 0.7297625009; H, -1.0199648502, -0.0145033354, -2.6249086483. Harmonic Frequencies (Number, Frequency $\left(\mathrm{cm}^{-1}\right)$, IR Intensity $\left(\mathrm{km} \mathrm{mol}^{-1}\right)$ ): 1, 108.7721, 1.5791; 2, 124.4521, 2.1751; 3, 353.9958, 21.0633; 4, 398.873, 32.8987; 5, 486.1289, 26.6690; 6, 530.815, 33.2012; 7, 535.1088, 152.1696; 8, 634.8359, 5.6920; 9, 653.3529, 94.1354; 10, 782.646, 96.3817; 11, 1017.0921, 216.7152; 12, 1119.2522, 52.2080; 13, 1184.8725, 456.9360; 14, 1414.0745, 41.3055; 15, 1738.3155, 716.3468; 16, 2206.747, 1468.7014; 17, 3118.675, 89.0101; 18, 3307.8577, 225.4319;

\section{Calculation summary for 1,3 Migration of Cl 1 sZ iE (acetonitrile)}

Geometry optimisation and frequency calculation: Program version = x86-Linux-G03RevB.01. Method and basis set $=$ RB3LYP/6-31G(d). Calculated Energy = -705.6530401 (hartree), $-2951.0410136982\left(\mathrm{~kJ} \mathrm{~mol}^{-1}\right)$. Higher level single-point calculation: Program = x86-Linux-G03RevB.01. Method and basis set $=$ RB3LYP/6$311++G(3 d f, 2 p)$. Calculated Energy = -705.7838294 (hartree), -2951.5879745508 $\left(\mathrm{kJ} \mathrm{mol}^{-1}\right)$. Structure definition (3D Coordinates: Atom, X coord/Å, Y coord/Å, Z coord/Å): C, 0.8070743442, 0.0896793828, 0.3856996185; C, 0.9124631429, 0.1195048776, 1.7107888781; H, 1.7408156944, 0.1774035179, 0.1690539702; O, 1.0201610968, 0.1466948118, 2.8680338428; C, -0.473965145, - 0.0518312665 , 0.2967801002; N, -1.6661917209, -0.166253878, 0.1020330256; Cl, -0.2333210129, -0.0573158832, 2.0835548014; H, -1.7457392558, -0.1569328155, 1.1327332947. Harmonic Frequencies (Number, Frequency $\left(\mathrm{cm}^{-1}\right)$, IR Intensity $\left(\mathrm{km} \mathrm{mol}^{-1}\right)$ ): 1, 47.5576, 15.1668; 2, 132.7434, 9.4334; 3, 317.5956, 1.3002; 4, 437.2997, 11.3489; 5, 501.0712, 26.9101; 6, 526.8319, 63.5392; 7, 538.2265, 24.3539; 8, 632.9426, 30.4099; 9, 756.2724, 338.9795; 10, 790.6787, 138.9791; 11, 986.0804, 117.0634; 12, 1146.784, 6.4117; 13, 1205.8562, 283.0991; 14, 1416.1772, 284.1679; 15, 1697.6949, 396.8862; 16, 2199.3586, 1395.5910; 17, 3129.3429, 96.4252; 18, 3248.2986, 83.5660;

\section{Calculation summary for 1,3 Migration of $\mathrm{Cl} 1 \mathrm{sZ}$ iZ (acetonitrile)}

Geometry optimisation and frequency calculation: Program version $=$ x86-Linux-G03RevB.01. Method and basis set $=$ RB3LYP/6-31G(d). Calculated Energy = -705.6584642 (hartree), $-2951.0636972844\left(\mathrm{~kJ} \mathrm{~mol}^{-1}\right)$. Higher level single-point calculation: Program = x86-Linux-G03RevB.01. Method and basis set $=$ RB3LYP/6$311++G(3 d f, 2 p)$. Calculated Energy = -705.7886083 (hartree), $-2951.6079599106\left(\mathrm{~kJ} \mathrm{~mol}^{-1}\right)$. Structure 
definition (3D Coordinates: Atom, X coord/Å, Y coord/Å, Z coord/Å): C, 0.8435465931, 0.0634214113, 0.3999995954; C, 0.8816702533, 0.040318679, 1.7299090863; H, 1.7893840919, 0.1536890973, 0.1298339757; O, 0.9300634049, 0.0214298199, 2.8909330364; C, -0.435221308, -0.0311303475, 0.2830281278; N, -1.5628033152, -0.1372655743, 0.2687231211; Cl, -0.2161833717, 0.0230193799, 2.0925529006; H, -2.3551240358, -0.1912565505, -0.386576176. Harmonic Frequencies (Number, Frequency

$\left(\mathrm{cm}^{-1}\right)$, IR Intensity $\left.\left(\mathrm{km} \mathrm{mol}^{-1}\right)\right): 1,64.6295,0.5446 ; 2,118.3372,7.3771 ; 3,310.9223,5.2725 ; 4,415.0154$, 54.3738; 5, 495.345, 80.8474; 6, 510.2789, 13.6479; 7, 534.2204, 34.3967; 8, 636.4496, 55.8551; 9, 767.1955, 154.7774; 10, 774.648, 249.0770; 11, 1010.6563, 89.4832; 12, 1139.0023, 1.3700; 13, 1155.1112, 554.2643; 14, 1415.8335, 285.9182; 15, 1723.4023, 376.4719; 16, 2201.5022, 1434.8694; 17, 3152.9285, 94.8907; 18, 3302.8633, 195.6354;

\section{1,3 Migration of $F$}

\section{Calculation summary for 1,3 Migration of F 3 sE (vacuum)}

Geometry optimisation and frequency calculation: Program version = x86-Linux-G03RevB.01. Method and basis set = RB3LYP/6-31G(d). Calculated Energy = -345.2975102 (hartree), -1444.0341876564 (kJ mol-1). Higher level single-point calculation: Program $=$ x86-Linux-G03RevB.01. Method and basis set $=$ RB3LYP/6$311+G(3 d f, 2 p)$. Calculated Energy = -345.4365889 (hartree), $-1444.6158147798\left(\mathrm{~kJ} \mathrm{~mol}^{-1}\right)$. Structure definition (3D Coordinates: Atom, X coord/Å, Y coord/Å, Z coord/Å): C, 0.7854364058, 0.116997333, 0.4064775249; C, 1.1355306577, -0.0214935607, 0.868156614; N, 1.4541533279, -0.0182028026, 2.0395929419; C, -0.6055417852, 0.0533816463, -0.8293318243; F, -1.4416576906, -0.1527495307, 0.2322923092; O, -1.0382472358, 0.1552490426, -1.9387892874; H, 1.543860222, 0.2632090151, 1.1666362843; H, 1.6654119142, -0.8963484725, 2.5150856181. Harmonic Frequencies (Number, Frequency $\left(\mathrm{cm}^{-1}\right)$, IR Intensity $\left.\left(\mathrm{km} \mathrm{mol}^{-1}\right)\right): 1,111.8293,3.5187 ; 2,151.1155,0.8758 ; 3,404.7027,1.7042 ; 4,479.4328$, 44.4798; 5, 519.0644, 39.5449; 6, 524.7189, 4.9368; 7, 686.0518, 14.9714; 8, 760.2714, 46.5986; 9, 846.6368, 36.3564; 10, 866.577, 75.8563; 11, 928.5504, 420.1434; 12, 1133.971, 37.8176; 13, 1242.9908, 205.1710; 14, 1413.5879, 32.7789; 15, 1896.0839, 563.4140; 16, 2157.2261, 486.7657; 17, 3239.4606, 7.5277; 18, 3502.4394, 46.5491;

\section{Calculation summary for 1,3 Migration of F 3 sZ (vacuum)}

Geometry optimisation and frequency calculation: Program version = x86-Linux-G03RevB.01. Method and basis set = RB3LYP/6-31G(d). Calculated Energy = -345.2954084 (hartree), -1444.0253979288 $\left(\mathrm{kJ} \mathrm{mol}^{-1}\right)$. Higher level single-point calculation: Program = x86-Linux-G03RevB.01. Method and basis set = RB3LYP/6$311+G(3 d f, 2 p)$. Calculated Energy = -345.4350341 (hartree), -1444.6093126062 (kJ mol $\left.{ }^{-1}\right)$. Structure definition (3D Coordinates: Atom, X coord/Å, Y coord/Å, Z coord/Å): C, 0.7923908877, 0.0452444372, 0.0771718308; C, 0.8082268948, 0.0934067207, 1.2515199774; H, 1.7243819159, 0.0810006631, 0.6289934614; N, 0.8222687767, 0.1373194628, 2.4629149273; C, -0.4798347089, -0.0230151145, 0.7870686761; O, -1.5917310231, -0.0578090406, -0.3456194407; F, -0.2579878487, -0.0606717394, 2.1304025952; H, 0.8507770288, -0.727535184, 3.00349103. Harmonic Frequencies (Number, Frequency (cm

1), IR Intensity $\left(\mathrm{km} \mathrm{mol}^{-1}\right)$ ): 1, 106.2624, 3.2389; 2, 147.1839, 1.6608; 3, 402.5035, 0.2145; 4, 469.2029, 61.8225; 5, 502.8239, 1.8155; 6, 517.2736, 41.8542; 7, 680.9773, 14.0733; 8, 749.367, 48.7416; 9, 841.4405, 62.4102; 10, 916.9945, 421.7670; 11, 956.2041, 9.3524; 12, 1144.713, 245.5358; 13, 1152.3523, 91.2006; 14, 1445.0862, 155.6864; 15, 1878.9019, 341.0253; 16, 2162.5954, 503.5385; 17, 3235.6864, 6.3198; 18, 3507.9881, 52.1799;

Calculation summary for 1,3 Migration of F TS2 iE (vacuum) 
Geometry optimisation and frequency calculation: Program version = x86-Linux-G03RevB.01. Method and basis set = RB3LYP/6-31G(d). Calculated Energy = -345.2436617 (hartree), -1443.8089932294 (kJ mol $\left.{ }^{-1}\right)$. Higher level single-point calculation: Program = x86-Linux-G03RevB.01. Method and basis set = RB3LYP/6-

$311+G(3 d f, 2 p)$. Calculated Energy = -345.3835588 (hartree), $-1444.3940429016\left(\mathrm{~kJ} \mathrm{~mol}^{-1}\right)$. Structure definition (3D Coordinates: Atom, X coord/Å, Y coord/A, Z coord/Å): C, 0.8038414125, 0.0313821108, 0.6715324189; C, 0.8754949955, 0.1018854439, 0.7042168833; O, 1.4635432046, 0.1961476154, 1.7124029381; C, -0.5463017126, -0.0929911185, -0.9787044941; N, -1.5738444691, -0.1978484977, 1.6024893265; F, -0.8227490103, -0.0135448748, 0.7941517047; H, 1.62457891, 0.0895845286, 1.3712345989; H, -1.7094803431, -0.3935807113, -2.5818087842. Harmonic Frequencies (Number, Frequency $\left(\mathrm{cm}^{-1}\right)$, IR Intensity $\left.\left(\mathrm{km} \mathrm{mol}^{-1}\right)\right): 1,-521.6931,221.9558 ; 2,122.8593,57.5641 ; 3,179.9461,75.6724 ; 4$, 300.8566, 7.4654; 5, 481.5238, 39.7480; 6, 554.4887, 43.1531; 7, 587.4442, 9.3486; 8, 595.1088, 15.7033; 9, 610.0764, 83.0785; 10, 658.9076, 35.4262; 11, 742.727, 204.6987; 12, 1065.3898, 0.6941; 13, 1179.5683, 46.6940; 14, 1354.5041, 20.1594; 15, 2058.298, 1507.1643; 16, 2140.3167, 126.1616; 17, 3283.907, 5.5453; 18, 3693.0642, 236.6840;

\section{Calculation summary for 1,3 Migration of F TS2 iZ (vacuum)}

Geometry optimisation and frequency calculation: Program version = x86-Linux-G03RevB.01. Method and basis set = RB3LYP/6-31G(d). Calculated Energy = -345.2519139 (hartree), -1443.8435039298 $\left(\mathrm{kJ} \mathrm{mol}^{-1}\right)$. Higher level single-point calculation: Program = x86-Linux-G03RevB.01. Method and basis set = RB3LYP/6311+G(3df,2p). Calculated Energy = -345.391339 (hartree), -1444.426579698 $\left(\mathrm{kJ} \mathrm{mol}^{-1}\right)$. Structure definition

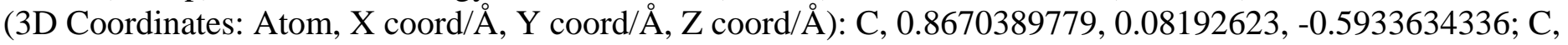
0.801475198, -0.0290766467, 0.7886848413; N, 1.2886099149, -0.0960001064, 1.8929430501; C, 0.4354169828, 0.0635132838, -1.0415860253; F, -1.0011070352, -0.0868859349, 0.585975608; O, 1.250707434, 0.096835737, -1.8800197178; H, 1.758928341, 0.1665014672, -1.1962114526; H, 0.8378418853, -0.1853904075, 2.7895750784. Harmonic Frequencies (Number, Frequency (cm ${ }^{-1}$ ), IR Intensity $\left.\left(\mathrm{km} \mathrm{mol}^{-1}\right)\right): 1,-482.471,207.4940 ; 2,60.8475,117.2377 ; 3,174.7193,69.5116 ; 4,285.8951,14.3340 ; 5$, 474.8885, 8.7422; 6, 570.2397, 23.9545; 7, 578.7209, 5.7897; 8, 613.7542, 7.4329; 9, 645.0968, 90.6451; 10, 654.6236, 50.7606; 11, 742.1883, 279.7679; 12, 1063.4628, 3.3863; 13, 1173.2261, 42.3840; 14, 1361.1651, 22.7233; 15, 2065.7118, 1431.7113; 16, 2147.8983, 140.5478; 17, 3288.1112, 8.9766; 18, 3705.1322, 258.7006;

\section{Calculation summary for 1,3 Migration of F $1 \mathrm{sE}$ iE (vacuum)}

Geometry optimisation and frequency calculation: Program version = x86-Linux-G03RevB.01. Method and basis set = RB3LYP/6-31G(d). Calculated Energy = -345.2890102 (hartree), -1443.9986406564 $\left(\mathrm{kJ} \mathrm{mol}^{-1}\right)$. Higher level single-point calculation: Program = x86-Linux-G03RevB.01. Method and basis set = RB3LYP/6$311+G(3 d f, 2 p)$. Calculated Energy = -345.4287479 (hartree), $-1444.5830237178\left(\mathrm{~kJ} \mathrm{~mol}^{-1}\right)$. Structure definition (3D Coordinates: Atom, X coord/Å, Y coord/Å, Z coord/Å): C, 0.8789407936, 0.0870320052, 0.1237013783; C, 0.823859266, -0.1460245396, 1.1838944979; O, 0.7832065748, -0.3492375366, 2.3278433161; C, -0.3444549353, 0.1351253376, -0.9181136898; N, -0.533586678, 0.3356934153, 2.1466276436; F, -1.4309624691, -0.0820204819, -0.1369605892; H, 1.8516263512, 0.2383039304, 0.5765226109; H, 0.3464192717, 0.4871299737, -2.6396616885. Harmonic Frequencies (Number, Frequency $\left(\mathrm{cm}^{-1}\right)$, IR Intensity $\left.\left(\mathrm{km} \mathrm{mol}^{-1}\right)\right): 1,105.6523,2.3256 ; 2,143.7415,3.6800 ; 3,408.6334,2.1255 ; 4,512.7937$, 7.2650; 5, 522.3711, 1.3853; 6, 540.4055, 19.9631; 7, 695.0284, 2.6741; 8, 695.339, 38.3307; 9, 837.8427, 81.8160; 10, 901.4521, 35.1556; 11, 1050.2238, 118.3389; 12, 1141.9974, 9.6488; 13, 1342.0098, 216.7056; 
14, 1401.8089, 50.8573; 15, 1772.6456, 365.6764; 16, 2244.3373, 779.4457; 17, 3238.6894, 10.5609; 18, 3511.2161, 6.8095;

\section{Calculation summary for 1,3 Migration of F 1 sE iZ (vacuum)}

Geometry optimisation and frequency calculation: Program version = x86-Linux-G03RevB.01. Method and basis set = RB3LYP/6-31G(d). Calculated Energy = -345.2927487 (hartree), -1444.0142750634 $\left(\mathrm{kJ} \mathrm{mol}^{-1}\right)$. Higher level single-point calculation: Program = x86-Linux-G03RevB.01. Method and basis set = RB3LYP/6311+G(3df,2p). Calculated Energy = -345.4316954 (hartree), $-1444.5953501628\left(\mathrm{~kJ} \mathrm{~mol}^{-1}\right)$. Structure definition (3D Coordinates: Atom, X coord/A, Y coord/A, Z coord/Å): C, 0.8828207067, -0.0573728047, 0.1577106188; C, -0.3513313256, -0.0081372715, -0.9184408612; N, -0.434137922, -0.0428520116, 2.1737787831; C, 0.8768335996, -0.0151403301, 1.1694840583; F, -1.4430313674, 0.082665378, 0.093800803; O, 0.893961655, 0.0206033001, 2.3322211272; H, 1.8315161062, -0.1301293191, 0.6752709067; H, -1.4068994702, 0.0051810363, -2.4818348721. Harmonic Frequencies (Number, Frequency $\left(\mathrm{cm}^{-1}\right)$, IR Intensity $\left.\left(\mathrm{km} \mathrm{mol}^{-1}\right)\right): 1,113.994,1.3851 ; 2,139.9335,1.7162 ; 3,412.2828,5.0275 ; 4,493.4057$, 29.8406; 5, 526.4102, 7.6247; 6, 534.5732, 46.2031; 7, 677.4642, 20.8718; 8, 701.0243, 27.2576; 9, 852.9988, 88.3395; 10, 867.3369, 38.8311; 11, 1064.1021, 118.9239; 12, 1129.3651, 2.7094; 13, 1309.7586, 114.8253; 14, 1418.9205, 46.1495; 15, 1788.1351, 401.9702; 16, 2246.2901, 779.1944; 17, 3248.3071, 20.7824; 18, 3501.6094, 15.8524;

\section{Calculation summary for 1,3 Migration of F $1 \mathrm{sZ}$ iE (vacuum)}

Geometry optimisation and frequency calculation: Program version = x86-Linux-G03RevB.01. Method and basis set = RB3LYP/6-31G(d). Calculated Energy = -345.2845795 (hartree), $-1443.980111469\left(\mathrm{~kJ} \mathrm{~mol}^{-1}\right)$. Higher level single-point calculation: Program = x86-Linux-G03RevB.01. Method and basis set $=$ RB3LYP/6311+G(3df,2p). Calculated Energy = -345.4252626 (hartree), $-1444.5684481932\left(\mathrm{~kJ} \mathrm{~mol}^{-1}\right)$. Structure definition (3D Coordinates: Atom, X coord/Å, Y coord/Å, Z coord/Å): C, 0.7738813466, 0.0764238301, 0.0802644863; C, 0.8825852582, 0.1385904815, 1.2393244482; H, 1.6886193202, 0.1480674889, 0.6585271791; O, 0.972907064, 0.1926805254, 2.3991909787; C, -0.512411299, -0.0824108259, 0.7703217002; N, -1.7038831151, -0.1894314743, -0.3719476399; F, -0.2929230708, -0.1113263448, 2.1010719988; H, -1.7727182249, -0.1571751827, 0.645851248. Harmonic Frequencies (Number, Frequency $\left(\mathrm{cm}^{-1}\right)$, IR Intensity $\left.\left(\mathrm{km} \mathrm{mol}^{-1}\right)\right): 1,63.4381,10.4809 ; 2,138.7229,6.9081 ; 3,410.4809,0.6233 ; 4,505.0021$, 15.4571; 5, 514.7245, 1.8858; 6, 539.9236, 16.1915; 7, 678.2945, 6.0427; 8, 682.9924, 47.9983; 9, 831.2167, 54.5158; 10, 936.5401, 35.9273; 11, 1047.2177, 174.3783; 12, 1138.5889, 0.1997; 13, 1277.8385, 127.5923; 14, 1448.6091, 190.5575; 15, 1754.5763, 239.2783; 16, 2236.3802, 657.5117; 17, 3226.5481, 18.5178; 18, 3498.9476, 4.5465;

\section{Calculation summary for 1,3 Migration of F 1 sZ iZ (vacuum)}

Geometry optimisation and frequency calculation: Program version = x86-Linux-G03RevB.01. Method and basis set = RB3LYP/6-31G(d). Calculated Energy = -345.2911903 (hartree), -1444.0077578346 (kJ mol $\left.{ }^{-1}\right)$. Higher level single-point calculation: Program = x86-Linux-G03RevB.01. Method and basis set = RB3LYP/6311+G(3df,2p). Calculated Energy = -345.4307388 (hartree), $-1444.5913496616\left(\mathrm{~kJ} \mathrm{~mol}^{-1}\right)$. Structure definition (3D Coordinates: Atom, X coord/Å, Y coord/Å, Z coord/Å): C, 0.8021318498, 0.0670811872, 0.0667587965; C, 0.8532521338, 0.0539926809, 1.260987775; H, 1.7315799031, 0.1509349395, 0.6162048906; O, 0.9254556226, 0.0447939473, 2.4206722001; C, -0.4742308223, -0.0291646881, 0.7585188301; N, -1.6068729527, -0.1293756167, -0.212380731; F, -0.2912911007, 0.0030118845, 2.1076606437; H, -2.3524132769, -0.1822192417, -0.9078226901. Harmonic Frequencies (Number, Frequency 
$\left(\mathrm{cm}^{-1}\right)$, IR Intensity $\left.\left(\mathrm{km} \mathrm{mol}^{-1}\right)\right): 1$, 92.7381, 0.0000; 2, 143.5918, 0.3757; 3, 404.0721, 3.0324; 4, 487.5609, 28.6549; 5, 510.1973, 17.9842; 6, 545.9333, 33.8355; 7, 650.378, 22.3543; 8, 669.1684, 8.0092; 9, 841.6001, 115.9100; 10, 960.3813, 24.1501; 11, 1058.5514, 114.0734; 12, 1139.4885, 2.7440; 13, 1256.1591, 155.6301; 14, 1465.3614, 166.4519; 15, 1768.0491, 274.3909; 16, 2247.9886, 684.2769; 17, 3245.871, 16.8620; 18, 3507.8664, 12.8414;

\section{Calculation summary for 1,3 Migration of F $3 s E$ (acetonitrile)}

Geometry optimisation and frequency calculation: Program version = x86-Linux-G03RevB.01. Method and basis set $=$ RB3LYP/6-31G(d). Calculated Energy = -345.3141182 (hartree), $-1444.1036423124\left(\mathrm{~kJ} \mathrm{~mol}^{-1}\right)$. Higher level single-point calculation: Program = x86-Linux-G03RevB.01. Method and basis set $=$ RB3LYP/6311++G(3df,2p). Calculated Energy = -345.4544666 (hartree), -1444.6905793212 $\left(\mathrm{kJ} \mathrm{mol}^{-1}\right)$. Structure definition (3D Coordinates: Atom, X coord/A, Y coord/A, Z coord/ $\AA$ ): C, 0.7854016656, 0.1201603296, 0.4105560974; C, 1.1337753392, -0.0236862758, 0.8711199978; N, 1.451440198, -0.0241347286, 2.0355260134; C, -0.591855582, 0.0539791621, -0.8269949952; F, -1.4438011694, -0.1461068849, 0.2298875429; O, -1.0460326873, 0.1516059862, -1.9367925882; H, 1.5537433674, 0.268339049, 1.1667258595; H, 1.6847187329, -0.9000011695, 2.5419831544. Harmonic Frequencies (Number, Frequency $\left(\mathrm{cm}^{-1}\right)$, IR Intensity $\left(\mathrm{km} \mathrm{mol}^{-1}\right)$ ): 1, 100.8209, 2.8204; 2, 149.3695, 2.9609; 3, 409.5399, 9.4413; 4, 478.7349, 95.4483; 5, 518.6232, 73.3306; 6, 529.8031, 19.3070; 7, 681.4999, 16.2344; 8, 754.7636, 74.4702; 9, 810.5608, 159.9519; 10, 816.7377, 628.5167; 11, 854.5421, 270.6264; 12, 1128.5774, 51.4840; 13, 1224.9437, 267.2423; 14, 1415.9595, 68.7146; 15, 1822.5834, 1409.3779; 16, 2150.9432, 850.3875; 17, 3154.2295, 274.7807; 18, 3162.1395, 345.2274;

\section{Calculation summary for 1,3 Migration of F 3 sZ (acetonitrile)}

Geometry optimisation and frequency calculation: Program version = x86-Linux-G03RevB.01. Method and basis set $=$ RB3LYP/6-31G(d). Calculated Energy = -345.3128218 (hartree), $-1444.0982207676\left(\mathrm{~kJ} \mathrm{~mol}^{-1}\right)$. Higher level single-point calculation: Program = x86-Linux-G03RevB.01. Method and basis set $=$ RB3LYP/6311++G(3df,2p). Calculated Energy = -345.4536999 (hartree), $-1444.6873729818\left(\mathrm{~kJ} \mathrm{~mol}^{-1}\right)$. Structure definition (3D Coordinates: Atom, X coord/A, Y coord/A, Z coord/ $\AA$ ): C, 0.7900200956, 0.0423174427, 0.0791898746; C, 0.8065462553, 0.0259132622, 1.2568175864; H, 1.7287710955, 0.0807370695, $0.6278197947 ;$ N, 0.8239622505, 0.1395718961, 2.4578121573; C, -0.4689785167, -0.0195658917, 0.7812898811; O, -1.5947641862, -0.0744917297, -0.3557615898; F, -0.2640280853, -0.0079234207, 2.1332411893; H, 0.8723324035, -0.6824845969, 3.0903711315. Harmonic Frequencies (Number, Frequency $\left(\mathrm{cm}^{-1}\right)$, IR Intensity $\left.\left(\mathrm{km} \mathrm{mol}^{-1}\right)\right): 1$, 95.0844, 2.2967; 2, 145.527, 6.6997; 3, 409.5847, 1.6013; 4, 474.4247, 100.4827; 5, 505.9461, 17.9225; 6, 520.0753, 74.1884; 7, 674.6116, 30.7747; 8, 744.2445, 79.4198; 9, 810.9412, 137.4994; 10, 817.2094, 818.0889; 11, 951.2631, 54.5609; 12, 1123.1875, 512.2877; 13, 1146.4912, 1.2186; 14, 1440.91, 302.1456; 15, 1808.8464, 725.5342; 16, 2151.2624, 868.1548; 17, 3153.3144, 319.4593; 18, 3161.2712, 282.0887;

\section{Calculation summary for 1,3 Migration of F TS2 iE (acetonitrile)}

Geometry optimisation and frequency calculation: Program version $=$ x86-Linux-G03RevB.01. Method and basis set $=$ RB3LYP/6-31G(d). Calculated Energy = -345.2597017 (hartree), $-1443.8760725094\left(\mathrm{~kJ} \mathrm{~mol}^{-1}\right)$. Higher level single-point calculation: Program = x86-Linux-G03RevB.01. Method and basis set $=$ RB3LYP/6$311++G(3 d f, 2 p)$. Calculated Energy = -345.4016827 (hartree), $-1444.4698370514\left(\mathrm{~kJ} \mathrm{~mol}^{-1}\right)$. Structure definition (3D Coordinates: Atom, X coord/Å, Y coord/Å, Z coord/Å): C, 0.8017807074, 0.0340096094, 0.6703743937; C, 0.9032857765, 0.1246308881, 0.6903294108; O, 1.4738906936, 0.2381809089, 
1.7103166706; C, -0.5534751079, -0.1094401391, -0.9757152349; N, -1.5724127406, -0.2407521021, 1.6001594745; F, -0.8459336395, -0.0297420058, 0.8062256298; H, 1.6184582341, 0.0668397142, 1.3846094371; H, -1.6988400998, -0.3145463679, -2.6182769679. Harmonic Frequencies (Number, Frequency $\left(\mathrm{cm}^{-1}\right)$, IR Intensity $\left.\left(\mathrm{km} \mathrm{mol}^{-1}\right)\right): 1,-520.0774,505.5183 ; 2,133.878,15.3913 ; 3,244.8536,125.9247 ; 4$, 263.3061, 15.5496; 5, 454.5027, 63.8655; 6, 523.2071, 46.0733; 7, 562.9548, 55.7421; 8, 580.3836, 11.9645; 9, 608.5075, 107.0708; 10, 637.745, 53.5741; 11, 704.0714, 336.4162; 12, 1058.0573, 2.2541; 13, 1154.9499, 70.0569; 14, 1352.8718, 40.0560; 15, 2011.3773, 2942.7912; 16, 2123.5811, 86.4256; 17, 3192.8444, 58.0634; 18, 3285.4014, 941.2613;

\section{Calculation summary for 1,3 Migration of F TS2 iZ (acetonitrile)}

Geometry optimisation and frequency calculation: Program version = x86-Linux-G03RevB.01. Method and basis set = RB3LYP/6-31G(d). Calculated Energy = -345.265476 (hartree), -1443.900220632 (kJ mol $\left.{ }^{-1}\right)$. Higher level single-point calculation: Program = x86-Linux-G03RevB.01. Method and basis set = RB3LYP/6311++G(3df,2p). Calculated Energy = -345.407127 (hartree), -1444.492605114 (kJ mol$\left.{ }^{-1}\right)$. Structure definition (3D Coordinates: Atom, X coord/A, Y coord/Å, Z coord/Å): C, 0.864282251, 0.0820442821, -0.5935222648; C, 0.7889889711, -0.030157484, 0.795755157; N, 1.2906390846, -0.094681371, 1.8889417457; C, 0.4157961725, 0.0670829999, -1.0703858007; F, -1.0097825055, -0.0899239199, 0.6112224001; O, 1.2507965917, 0.0975601222, -1.8931532665; H, 1.7682875446, 0.1652419395, -1.188601382; H, 0.86680385, -0.1874568286, 2.8191511439. Harmonic Frequencies (Number, Frequency $\left(\mathrm{cm}^{-1}\right.$ ), IR Intensity $\left(\mathrm{km} \mathrm{mol}^{-1}\right)$ ): 1, -506.004, 457.2912; 2, 137.8838, 7.2560; 3, 262.9726, 258.9747; 4, 266.8258, 37.6068; 5, 465.5576, 7.2082; 6, 535.3613, 141.2647; 7, 569.6849, 11.1369; 8, 601.7086, 107.2293; 9, 611.3038, 5.4662; 10, 634.2992, 70.4021; 11, 682.9532, 426.9462; 12, 1058.7974, 11.3357; 13, 1152.2736, 47.4316; 14, 1359.5776, 41.9765; 15, 2019.7714, 2781.5133; 16, 2131.9158, 102.5934; 17, 3191.388, 68.2669; 18, 3319.3476, 887.8142;

\section{Calculation summary for 1,3 Migration of F $1 \mathrm{sE}$ iE (acetonitrile)}

Geometry optimisation and frequency calculation: Program version = x86-Linux-G03RevB.01. Method and basis set = RB3LYP/6-31G(d). Calculated Energy = -345.302617 (hartree), -1444.055544294 (kJ mol $\left.{ }^{-1}\right)$. Higher level single-point calculation: Program = x86-Linux-G03RevB.01. Method and basis set = RB3LYP/6311++G(3df,2p). Calculated Energy = -345.4433261 (hartree), -1444.6439897502 (kJ mol $\left.{ }^{-1}\right)$. Structure definition (3D Coordinates: Atom, X coord/Å, Y coord/Å, Z coord/Å): C, 0.877446676, 0.0869216084, 0.1247998305; C, 0.8264089109, -0.1454336532, 1.1821664451; O, 0.7870583464, -0.3486727782, 2.3269089659; C, -0.3397476071, 0.1349123839, -0.9153640963; N, -0.53412345, 0.3353921986, 2.144633117; F, -1.4392902749, -0.082682213, -0.1370688613; H, 1.8545780737, 0.2393974586, 0.5819102819; H, 0.3567839013, 0.4879772591, -2.6393249843. Harmonic Frequencies (Number, Frequency $\left(\mathrm{cm}^{-1}\right)$, IR Intensity $\left(\mathrm{km} \mathrm{mol}^{-1}\right)$ ): 1, 105.6216, 4.2813; 2, 137.1436, 4.5984; 3, 410.3377, 2.5683; 4, 511.8871, 10.1109; 5, 527.2655, 0.5426; 6, 539.6481, 32.5684; 7, 691.905, 6.2762; 8, 697.1549, 56.2050; 9, 823.8297, 133.2598; 10, 879.4982, 105.2111; 11, 1057.1376, 142.5931; 12, 1128.2988, 28.5747; 13, 1322.9321, 372.2263; 14, 1401.1274, 78.9923; 15, 1745.3316, 829.2503; 16, 2206.3897, 1451.3809; 17, 3137.6443, 76.7563; 18, 3302.1946, 125.3085;

\section{Calculation summary for 1,3 Migration of F $1 \mathrm{sE}$ iZ (acetonitrile)}

Geometry optimisation and frequency calculation: Program version = x86-Linux-G03RevB.01. Method and basis set = RB3LYP/6-31G(d). Calculated Energy = -345.3031802 (hartree), -1444.0578995964 $\left(\mathrm{kJ} \mathrm{mol}^{-1}\right)$. Higher level single-point calculation: Program = x86-Linux-G03RevB.01. Method and basis set = RB3LYP/6- 
311++G(3df,2p). Calculated Energy = -345.4430714 (hartree), -1444.6429245948 (kJ mol $\left.{ }^{-1}\right)$. Structure definition (3D Coordinates: Atom, X coord/Å, Y coord/Å, Z coord/Å): C, 0.8880124746, -0.0579429954, 0.1572982621; C, -0.342524769, -0.0088871689, -0.9194310481; N, -0.4393725451, -0.0422320943, 2.1757465742; C, 0.8755062684, -0.0149664175, 1.1696499176; F, -1.4376551901, 0.0817404714, 0.0950860776; O, 0.8831392267, 0.0218300522, 2.3327678206; H, 1.850126367, -0.1310331069, 0.6619626184; H, -1.4266994981, 0.0071325978, -2.4717028735. Harmonic Frequencies (Number, Frequency $\left(\mathrm{cm}^{-1}\right)$, IR Intensity $\left.\left(\mathrm{km} \mathrm{mol}^{-1}\right)\right): 1,111.4882,2.6328 ; 2,133.3472,2.8595 ; 3,411.5468,7.8689 ; 4,492.9131$, 38.3855; 5, 518.875, 27.1596; 6, 533.1295, 61.8878; 7, 673.189, 34.1530; 8, 693.5902, 33.3677; 9, 825.4147, 138.3328; 10, 855.0386, 81.6509; 11, 1063.9753, 202.5082; 12, 1119.3145, 3.3277; 13, 1286.4088, 183.5791; 14, 1414.179, 65.4657; 15, 1757.3166, 861.2200; 16, 2208.9958, 1436.7268; 17, 3144.9645, 86.2172; 18, 3285.5358, 159.4518;

\section{Calculation summary for 1,3 Migration of F 1 sZ iE (acetonitrile)}

Geometry optimisation and frequency calculation: Program version = x86-Linux-G03RevB.01. Method and basis set = RB3LYP/6-31G(d). Calculated Energy = -345.2981486 (hartree), -1444.0368574452 (kJ mol $\left.{ }^{-1}\right)$. Higher level single-point calculation: Program = x86-Linux-G03RevB.01. Method and basis set = RB3LYP/6311++G(3df,2p). Calculated Energy = -345.439828 (hartree), -1444.629360696 $\left(\mathrm{kJ} \mathrm{mol}^{-1}\right)$. Structure definition (3D Coordinates: Atom, X coord/Å, Y coord/A, Z coord $/ \AA$ ): C, 0.7615613026, 0.0744871116, -0.0754827684; C, 0.8807006307, 0.138572523, 1.24421758; H, 1.6858064586, 0.1464263553, -0.6497179156; O, 1.0005845746, 0.1960535233, 2.4000446976; C, -0.515405652, -0.0825962875, -0.7628272647; N, 1.7151204303, -0.191440593, -0.3850967271; F, -0.2931854506, -0.1105785831, -2.1033790598; H, 1.8071086759, -0.1623432252, 0.6400036817. Harmonic Frequencies (Number, Frequency $\left(\mathrm{cm}^{-1}\right.$ ), IR Intensity $\left(\mathrm{km} \mathrm{mol}^{-1}\right)$ ): 1, 74.2123, 18.1053; 2, 132.2842, 10.2357; 3, 417.548, 0.3371; 4, 497.2231, 25.0634; 5, 515.1642, 5.3627; 6, 542.2264, 27.9271; 7, 675.7463, 3.7802; 8, 680.6819, 69.4698; 9, 808.0082, 94.4454; 10, 923.4847, 107.2618; 11, 1044.2501, 278.2187; 12, 1132.0044, 0.4701; 13, 1271.7073, 260.1945; 14, 1444.4455, 307.2838; 15, 1731.1613, 465.8629; 16, 2205.7702, 1199.6445; 17, 3121.8317, 94.2861; 18, 3313.5841, 65.6307;

\section{Calculation summary for 1,3 Migration of F 1 sZ iZ (acetonitrile)}

Geometry optimisation and frequency calculation: Program version = x86-Linux-G03RevB.01. Method and basis set = RB3LYP/6-31G(d). Calculated Energy = -345.3013156 (hartree), -1444.0501018392 (kJ mol $\left.{ }^{-1}\right)$. Higher level single-point calculation: Program = x86-Linux-G03RevB.01. Method and basis set $=$ RB3LYP/6-

311++G(3df,2p). Calculated Energy = -345.4417801 (hartree), -1444.6375243782 (kJ mol $\left.{ }^{-1}\right)$. Structure definition (3D Coordinates: Atom, X coord/A, Y coord/A, Z coord/Å): C, 0.8022170322, 0.0670407249, 0.0676166058; C, 0.8636262997, 0.0549704544, 1.2582339555; H, 1.7366171953, 0.1512145122, 0.6200060587; O, 0.9324339207, 0.0455187909, 2.4194942891; C, -0.4765192943, -0.029597766, 0.7511190586; N, -1.6160985743, -0.1304833132, -0.2159768691; F, -0.2958620316, 0.0028449257, 2.1033130075; H, -2.3565844816, -0.1820664577, -0.9312828492. Harmonic Frequencies (Number, Frequency $\left(\mathrm{cm}^{-1}\right)$, IR Intensity $\left.\left(\mathrm{km} \mathrm{mol}^{-1}\right)\right): 1,86.6829,0.0107 ; 2,126.3473,3.1430 ; 3,404.9087,5.1136 ; 4,488.1735$, 40.8528; 5, 507.4166, 22.7802; 6, 540.9381, 58.0804; 7, 647.763, 34.4214; 8, 664.755, 7.8103; 9, 813.335, 179.1307; 10, 948.942, 44.1335; 11, 1048.7636, 197.5975; 12, 1127.9899, 8.7551; 13, 1233.8111, 293.5938; 14, 1449.435, 248.9366; 15, 1740.4168, 495.2698; 16, 2207.1827, 1234.3805; 17, 3144.5145, 92.3640; 18, 3298.7852, 137.8671;

\section{1,3 Migration of $\mathrm{H}$}




\section{Calculation summary for 1,3 Migration of H 3 sE (vacuum)}

Geometry optimisation and frequency calculation: Program version = x86-Linux-G03RevB.01. Method and basis set = RB3LYP/6-31G(d). Calculated Energy = -246.0385192 (hartree), -1028.9330872944 $\left(\mathrm{kJ} \mathrm{mol}^{-1}\right)$. Higher level single-point calculation: Program = x86-Linux-G03RevB.01. Method and basis set = RB3LYP/6$311+G(3 d f, 2 p)$. Calculated Energy = -246.1371584 (hartree), $-1029.3455964288\left(\mathrm{~kJ} \mathrm{~mol}^{-1}\right)$. Structure definition (3D Coordinates: Atom, X coord/Å, Y coord/Å, Z coord/Å): C, 0.4601115027, 0.0895034779, 0.3483006594; C, 0.821474903, -0.0466996195, 0.9216358197; N, 1.1073606261, -0.0507718821, 2.106523464; C, -0.9490690236, 0.0089714303, -0.7499869311; H, -1.6660894393, -0.1521105183, 0.082340591; O, -1.3280241214, 0.1106769677, -1.9016529453; H, 1.2137898829, 0.2514356903, 1.1129250261; H, 1.3298638519, -0.9399894717, 2.5580543742. Harmonic Frequencies (Number, Frequency $\left(\mathrm{cm}^{-1}\right)$, IR Intensity $\left.\left(\mathrm{km} \mathrm{mol}^{-1}\right)\right): 1,153.0171,6.8357 ; 2,170.3106,3.6597 ; 3,484.3873,29.3547 ; 4,491.3468$, 27.6825; 5, 585.5789, 3.5662; 6, 595.5088, 73.9207; 7, 862.0902, 64.8302; 8, 956.8032, 417.8502; 9, 1016.0669, 2.9229; 10, 1103.5621, 77.2132; 11, 1148.7114, 31.1341; 12, 1352.312, 6.2420; 13, 1464.5993, 6.2375; 14, 1784.3787, 412.6789; 15, 2135.7089, 517.5160; 16, 2931.3472, 98.6177; 17, 3216.627, 3.6998; 18, 3481.3438, 29.9795;

\section{Calculation summary for 1,3 Migration of H 3 sZ (vacuum)}

Geometry optimisation and frequency calculation: Program version = x86-Linux-G03RevB.01. Method and basis set = RB3LYP/6-31G(d). Calculated Energy = -246.036246 (hartree), -1028.923580772 (kJ mol $\left.{ }^{-1}\right)$. Higher level single-point calculation: Program = x86-Linux-G03RevB.01. Method and basis set = RB3LYP/6311+G(3df,2p). Calculated Energy = -246.1344667 (hartree), -1029.3343397394 (kJ mol $\left.{ }^{-1}\right)$. Structure definition (3D Coordinates: Atom, X coord/Å, Y coord/A, Z coord/Å): C, 0.7331214595, 0.0332447306, 0.5577171182; C, 0.7511514846, 0.0836849751, 0.7714652104; H, 1.6747369917, 0.0664428553, 1.0974142767; N, 0.7678526181, 0.1297538856, 1.9844263753; C, -0.5329086424, -0.0433566162, 1.2984586503; O, -1.6441892633, -0.0745611644, -0.8009569581; H, -0.3962056803, -0.0825163921, 2.3998220193; H, 0.7918286584, -0.7371528842, 2.5221706824. Harmonic Frequencies (Number, Frequency $\left(\mathrm{cm}^{-1}\right)$, IR Intensity ( $\left.\mathrm{km} \mathrm{mol}^{-1}\right)$ ): 1, 150.0599, 3.2110; 2, 211.213, 12.3696; 3, 440.9627, 1.5753; 4, 471.9858, 60.7464; 5, 550.4887, 56.5743; 6, 792.0649, 66.6055; 7, 840.359, 64.4473; 8, 924.7493, 404.8254; 9, 948.2652, 11.2865; 10, 1019.2741, 3.1758; 11, 1149.6462, 0.2614; 12, 1424.109, 117.8954; 13, 1441.992, 5.5263; 14, 1776.3032, 247.1278; 15, 2146.7399, 566.2925; 16, 2933.497, 155.1659; 17, 3210.4496, 0.8235; 18, 3501.8439, 42.2084;

\section{Calculation summary for 1,3 Migration of H TS2 iE (vacuum)}

Geometry optimisation and frequency calculation: Program version = x86-Linux-G03RevB.01. Method and basis set = RB3LYP/6-31G(d). Calculated Energy = -245.9593792 (hartree), -1028.6021238144 $\left(\mathrm{kJ} \mathrm{mol}^{-1}\right)$. Higher level single-point calculation: Program = x86-Linux-G03RevB.01. Method and basis set = RB3LYP/6-

311+G(3df,2p). Calculated Energy = -246.0596334 (hartree), -1029.0213868788 (kJ mol $\left.{ }^{-1}\right)$. Structure definition (3D Coordinates: Atom, X coord $/ \AA$, Y coord/Å, Z coord/A): C, 0.8904980718, 0.0556686101, 0.117877832; C, -0.2072580909, 0.1286877858, -0.987624886; N, -0.8339604613, 0.2235612673, 2.0328209117; C, 0.136000435, -0.1211371429, 1.0329477606; O, 0.0590431586, -0.3331708813, 2.1998223529; H, 1.9586887155, 0.0976369097, -0.3018133767; H, -0.489783516, 0.6651148854, 2.8785879897; H, -1.0189697337, -0.0416291333, 0.2468986696. Harmonic Frequencies (Number, Frequency $\left(\mathrm{cm}^{-1}\right)$, IR Intensity $\left.\left(\mathrm{km} \mathrm{mol}^{-1}\right)\right): 1,-1230.267,1288.7506 ; 2,241.1132,56.2402 ; 3,254.5886,11.8585 ; 4$, 343.4449, 185.6937; 5, 564.3651, 15.4241; 6, 624.379, 6.1698; 7, 726.4357, 54.9029; 8, 747.3283, 80.3427; 9, 785.6785, 102.8219; 10, 1009.0191, 3.6717; 11, 1126.6113, 38.0353; 12, 1185.9164, 19.9211; 13, 1338.9139, 
0.6387; 14, 1739.1859, 48.2046; 15, 1998.8768, 1014.2729; 16, 2026.3743, 55.0525; 17, 3234.2531, 1.1473; 18, 3587.2325, 98.6178;

\section{Calculation summary for 1,3 Migration of H TS2 iZ (vacuum)}

Geometry optimisation and frequency calculation: Program version = x86-Linux-G03RevB.01. Method and basis set = RB3LYP/6-31G(d). Calculated Energy = -245.967553 (hartree), -1028.636306646 $\left(\mathrm{kJ} \mathrm{mol}^{-1}\right)$. Higher level single-point calculation: Program = x86-Linux-G03RevB.01. Method and basis set = RB3LYP/6311+G(3df,2p). Calculated Energy = -246.067485 (hartree), -1029.05422227 $\left(\mathrm{kJ} \mathrm{mol}^{-1}\right)$. Structure definition (3D Coordinates: Atom, X coord/A, Y coord/A, Z coord/A): C, 0.8699505269, -0.05129793, -0.2019387537; C, -0.299363589, -0.0201451834, -0.9715197437; N, -0.863502503, -0.0856265537, -2.059419881; C, 0.229672434, 0.0237443162, 1.0248528125; O, 0.2698899763, 0.1000043083, 2.2115109086; H, 1.9131631778, -0.1011758254, -0.4911660938; H, -1.8289969724, 0.1388847345, -2.2531470519; H, 1.0003247263, 0.0478352838, 0.3597991527. Harmonic Frequencies (Number, Frequency $\left(\mathrm{cm}^{-1}\right.$ ), IR Intensity (km mol$\left.{ }^{-1}\right)$ ): 1, -1204.8164, 1424.0056; 2, 235.3791, 149.4512; 3, 256.8759, 14.3029; 4, 276.8622, 43.7992; 5, 580.2162, 24.8567; 6, 618.847, 34.3269; 7, 667.68, 101.1799; 8, 733.5262, 72.1841; 9, 906.3312, 71.6563; 10, 1000.6399, 3.1668; 11, 1136.8763, 1.4072; 12, 1187.4109, 22.8937; 13, 1338.1477, 1.1675; 14, 1701.4311, 51.9508; 15, 1974.399, 750.0075; 16, 2018.4261, 247.2547; 17, 3246.892, 0.2708; 18, 3658.1024, 154.6997;

\section{Calculation summary for 1,3 Migration of $\mathrm{H} 1 \mathrm{sE} \mathrm{iE} \mathrm{(vacuum)}$}

Geometry optimisation and frequency calculation: Program version = x86-Linux-G03RevB.01. Method and basis set = RB3LYP/6-31G(d). Calculated Energy = -246.043772 (hartree), -1028.955054504 (kJ mol-1). Higher level single-point calculation: Program = x86-Linux-G03RevB.01. Method and basis set = RB3LYP/6311+G(3df,2p). Calculated Energy = -246.141722 (hartree), -1029.364681404 (kJ mol$\left.{ }^{-1}\right)$. Structure definition (3D Coordinates: Atom, X coord/Å, Y coord/Å, Z coord/Å): C, 0.5233300543, 0.0637403234, -0.1494623908; C, 0.521438167, -0.1592961926, 1.1586384426; O, 0.4977678137, -0.3568477153, 2.3080043925; C, 0.7178884186, 0.106483657, -0.9317527006; N, -0.8387206415, 0.311165423, -2.1873403209; H, 1.6392615129, -0.0567422351, -0.3652916802; H, 1.4885917669, 0.2150898683, -0.6242617789; H, 0.0782929113, 0.4527094019, -2.6276395419. Harmonic Frequencies (Number, Frequency (cm ${ }^{-1}$ ), IR Intensity $\left.\left(\mathrm{km} \mathrm{mol}^{-1}\right)\right):$ 1, 132.6605, 0.1652; 2, 167.8329, 8.3989; 3, 467.8516, 3.7305; 4, 532.7044, 10.4646; 5, 584.6658, 20.1932; 6, 618.7824, 8.2675; 7, 831.1545, 48.4394; 8, 1053.6789, 17.2241; 9, 1114.9512, 53.3181; 10, 1142.5251, 3.3028; 11, 1261.0682, 190.7286; 12, 1380.8234, 11.9523; 13, 1456.2841, 20.3681; 14, 1693.1423, 231.4160; 15, 2225.5802, 835.1525; 16, 3117.944, 25.3144; 17, 3206.3333, 4.7147; 18, 3400.2433, 9.3653;

\section{Calculation summary for 1,3 Migration of H $1 \mathrm{sE} \mathrm{iZ} \mathrm{(vacuum)}$}

Geometry optimisation and frequency calculation: Program version = x86-Linux-G03RevB.01. Method and basis set = RB3LYP/6-31G(d). Calculated Energy = -246.0442188 (hartree), $-1028.9569230216\left(\mathrm{~kJ} \mathrm{~mol}^{-1}\right)$. Higher level single-point calculation: Program = x86-Linux-G03RevB.01. Method and basis set = RB3LYP/6311+G(3df,2p). Calculated Energy = -246.1432248 (hartree), $-1029.3709661136\left(\mathrm{~kJ} \mathrm{~mol}^{-1}\right)$. Structure definition (3D Coordinates: Atom, X coord/Å, Y coord/Å, Z coord/Å): C, 0.5163472086, -0.0396844116, 0.175543282; C, -0.7434899608, 0.0144482901, -0.9105109815; N, -0.7491444476, -0.0317980878, 2.1895476558; C, 0.5776961596, 0.0047744035, 1.1479778487; H, -1.6478937214, 0.0948710011, 0.2927298988; O, 0.6311533921, 0.0440956862, 2.3135163127; H, 1.4503502963, -0.1196258401, 0.7238375838; H, -1.7109930228, 0.0173462726, -2.5362709389. Harmonic Frequencies (Number, Frequency 
$\left(\mathrm{cm}^{-1}\right)$, IR Intensity $\left.\left(\mathrm{km} \mathrm{mol}^{-1}\right)\right): 1,146.4467,8.8236 ; 2,171.0725,9.5342 ; 3,473.8002,20.3867 ; 4,524.8227$, 10.5695; 5, 612.2493, 102.3785; 6, 623.13, 7.0239; 7, 808.252, 16.4212; 8, 1042.813, 102.4228; 9, 1085.2883, 6.9431; 10, 1122.9506, 3.8343; 11, 1283.7031, 18.5823; 12, 1371.4624, 25.5586; 13, 1460.1634, 17.2432; 14, 1693.7468, 226.4913; 15, 2226.661, 831.5684; 16, 3049.2162, 48.2623; 17, 3214.6281, 13.7116; 18, 3449.2262, 0.4536;

\section{Calculation summary for 1,3 Migration of H $1 \mathrm{sZ}$ iE (vacuum)}

Geometry optimisation and frequency calculation: Program version = x86-Linux-G03RevB.01. Method and basis set = RB3LYP/6-31G(d). Calculated Energy = -246.0407367 (hartree), -1028.9423608794 $\left(\mathrm{kJ} \mathrm{mol}^{-1}\right)$. Higher level single-point calculation: Program = x86-Linux-G03RevB.01. Method and basis set $=$ RB3LYP/6-

311+G(3df,2p). Calculated Energy = -246.1385373 (hartree), -1029.3513629886 (kJ mol $\left.{ }^{-1}\right)$. Structure

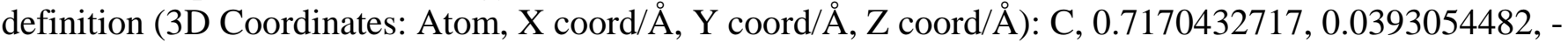
0.555928391; C, 0.8199718885, 0.117903416, 0.7641313537; H, 1.643746339, 0.0981492799, -1.1182926548; $\mathrm{O}, 0.8845917685,0.1846237995,1.9267657051$; C, -0.5641248748, -0.1197637764, -1.2668229659; N, 1.7604603825, -0.210659249, -0.8271740987; H, -0.4371861443, -0.1644766588, -2.3524411859; H, 1.7974133773, -0.1607188016, 0.1985469097. Harmonic Frequencies (Number, Frequency $\left(\mathrm{cm}^{-1}\right)$, IR Intensity $\left.\left(\mathrm{km} \mathrm{mol}^{-1}\right)\right): 1,133.5076,5.5713 ; 2,139.9211,4.9759 ; 3$, 463.6549, 1.6682; 4, 532.335, 1.7174; 5, 561.5081, 38.1087; 6, 743.3308, 26.9103; 7, 824.2563, 47.7128; 8, 931.5162, 16.4452; 9, 1118.9544, 45.3802; 10, 1143.9618, 0.4583; 11, 1246.5661, 83.5372; 12, 1415.7629, 130.9929; 13, 1444.7492, 1.8846; 14, 1689.3776, 142.6391; 15, 2218.9131, 682.7590; 16, 3120.2371, 35.8407; 17, 3213.6448, 7.5197; 18, 3389.1717, 11.9403;

\section{Calculation summary for 1,3 Migration of H 1 sZ iZ (vacuum)}

Geometry optimisation and frequency calculation: Program version = x86-Linux-G03RevB.01. Method and basis set = RB3LYP/6-31G(d). Calculated Energy = -246.0435681 (hartree), -1028.9542017942 (kJ mol $\left.{ }^{-1}\right)$. Higher level single-point calculation: Program = x86-Linux-G03RevB.01. Method and basis set = RB3LYP/6$311+G(3 d f, 2 p)$. Calculated Energy = -246.1422253 (hartree), -1029.3667862046 ( $\left.\mathrm{kJ} \mathrm{mol}^{-1}\right)$. Structure

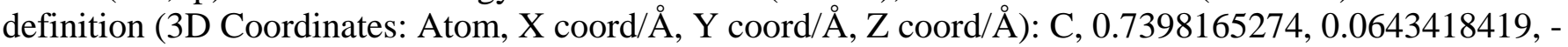
0.5453441908; C, 0.7856303202, 0.0562584799, 0.7838161602; H, 1.6843710004, 0.1447884558, 1.0720144549; O, 0.843212438, 0.0506364297, 1.9459292961; C, -0.5284259885, -0.0312872406, 1.2641239596; N, -1.654934481, -0.1273538724, -0.6632131258; H, -0.4106992276, -0.0119145194, 2.3561286566; H, -2.4169550644, -0.1823667544, -1.3428874354. Harmonic Frequencies (Number, Frequency $\left(\mathrm{cm}^{-1}\right)$, IR Intensity $\left.\left(\mathrm{km} \mathrm{mol}^{-1}\right)\right): 1,149.2453,0.3675 ; 2,181.4781,21.0349 ; 3,449.7454,16.9892 ; 4$, 534.3895, 13.2679; 5, 567.3198, 85.9249; 6, 754.517, 34.8336; 7, 793.6984, 25.2270; 8, 970.9768, 19.1025; 9, 1086.285, 9.5008; 10, 1142.4755, 0.8161; 11, 1224.564, 58.7757; 12, 1421.6571, 52.8736; 13, 1461.5979, 57.9938; 14, 1692.8481, 150.8241; 15, 2230.8174, 714.2568; 16, 3051.3005, 67.6306; 17, 3227.5126, 6.5550; 18, 3461.1743, 0.1379;

\section{Calculation summary for 1,3 Migration of $\mathrm{H} 3 \mathrm{sE}$ (acetonitrile)}

Geometry optimisation and frequency calculation: Program version = x86-Linux-G03RevB.01. Method and basis set = RB3LYP/6-31G(d). Calculated Energy = -246.0556642 (hartree), -1029.0047876844 (kJ mol $\left.{ }^{-1}\right)$. Higher level single-point calculation: Program = x86-Linux-G03RevB.01. Method and basis set = RB3LYP/6311++G(3df,2p). Calculated Energy = -246.1560668 (hartree), -1029.4246713576 $\left(\mathrm{kJ} \mathrm{mol}^{-1}\right)$. Structure definition (3D Coordinates: Atom, X coord/Å, Y coord/Å, Z coord/Å): C, 0.4586898108, 0.0893502612, 0.3547190985; C, 0.8158042426, -0.0491880359, 0.9236225548; N, 1.1080961609, -0.0533318619, 
2.0997098842; C, -0.9387425831, 0.0082843813, -0.7436850313; H, -1.6547544692, -0.1606858233, 0.0880099524; O, -1.3352482914, 0.1172295638, -1.9010586335; H, 1.2268687661, 0.2483075512, 1.1114537734; H, 1.3386900866, -0.942814845, 2.5826331499. Harmonic Frequencies (Number, Frequency

$\left(\mathrm{cm}^{-1}\right)$, IR Intensity $\left.\left(\mathrm{km} \mathrm{mol}^{-1}\right)\right): 1,158.7474,7.5379 ; 2,173.4061,7.9481 ; 3,488.726,131.0673 ; 4,492.7143$, 14.6546; 5, 584.635, 81.8704; 6, 589.1167, 43.2336; 7, 826.1392, 108.1220; 8, 858.2382, 856.9998; 9, 1025.85, 2.0792; 10, 1111.2272, 100.1400; 11, 1149.6197, 105.9495; 12, 1355.382, 38.4200; 13, 1463.6914, 16.0962; 14, 1708.5985, 1018.2341; 15, 2127.2734, 982.8963; 16, 2919.0526, 71.6977; 17, 3138.81, 42.9190; 18, 3166.1963, 473.6125;

\section{Calculation summary for 1,3 Migration of H 3 sZ (acetonitrile)}

Geometry optimisation and frequency calculation: Program version = x86-Linux-G03RevB.01. Method and basis set = RB3LYP/6-31G(d). Calculated Energy = -246.0539119 (hartree), -1028.9974595658 (kJ mol $\left.{ }^{-1}\right)$. Higher level single-point calculation: Program = x86-Linux-G03RevB.01. Method and basis set = RB3LYP/6311++G(3df,2p). Calculated Energy = -246.1537612 (hartree), -1029.4150293384 (kJ mol $\left.{ }^{-1}\right)$. Structure definition (3D Coordinates: Atom, X coord/Å, Y coord/A, Z coord/Å): C, 0.7329455384, 0.0404068744, 0.5654420004; C, 0.7570879094, 0.0300148216, 0.7699424887; H, 1.6754276336, 0.0812909303, 1.1099802455; N, 0.7562534445, 0.1463878202, 1.9741793405; C, -0.5244246347, -0.0250795598, 1.2954578688; O, -1.6454994069, -0.0849207844, -0.7952565452; H, -0.3983645149, -0.0159036759, 2.3981368597; H, 0.7995051469, -0.6827885378, 2.5966583671. Harmonic Frequencies (Number, Frequency $\left(\mathrm{cm}^{-1}\right)$, IR Intensity $\left.\left(\mathrm{km} \mathrm{mol}^{-1}\right)\right): 1,152.2343,9.2036 ; 2,216.8535,22.7155 ; 3,448.5375,2.9046 ; 4,483.3129$, 113.0185; 5, 552.5354, 88.8215; 6, 785.9458, 130.6956; 7, 818.7409, 104.8614; 8, 839.5726, 787.0948; 9, 963.0621, 52.9331; 10, 1029.4155, 3.1365; 11, 1143.2809, 1.2176; 12, 1421.1271, 302.0245; 13, 1435.1772, 24.4897; 14, 1705.785, 513.1385; 15, 2127.2603, 1024.8494; 16, 2927.6848, 133.6625; 17, 3145.3382, 48.2929; 18, 3169.4058, 466.3635;

\section{Calculation summary for 1,3 Migration of H TS2 iE (acetonitrile)}

Geometry optimisation and frequency calculation: Program version $=$ x86-Linux-G03RevB.01. Method and basis set = RB3LYP/6-31G(d). Calculated Energy = -245.9683088 (hartree), -1028.6394674016 (kJ mol $\left.{ }^{-1}\right)$. Higher level single-point calculation: Program = x86-Linux-G03RevB.01. Method and basis set = RB3LYP/6311++G(3df,2p). Calculated Energy = -246.0688186 (hartree), -1029.0597993852 (kJ mol $\left.{ }^{-1}\right)$. Structure definition (3D Coordinates: Atom, X coord/Å, Y coord/A, Z coord/Å): C, 0.9044884072, 0.0545766273, 0.1199422851; C, -0.2046623491, 0.1459078056, -0.9807115125; N, -0.8577047349, 0.2678766881, 2.0063727164; C, 0.1434497654, -0.1413700011, 1.0148276; O, 0.0540179051, -0.3589866252, 2.1877909175; H, 1.9701853302, 0.104139313, -0.3097850908; H, -0.4528541964, 0.5885202216, 2.8840225316; H, -1.005196171, -0.0505899401, 0.2510464823. Harmonic Frequencies (Number, Frequency $\left(\mathrm{cm}^{-1}\right)$, IR Intensity $\left.\left(\mathrm{km} \mathrm{mol}^{-1}\right)\right): 1,-1253.491,3119.9428 ; 2,162.7693,216.4133 ; 3,249.9425,8.1707 ; 4$, 277.1189, 33.0476; 5, 576.6278, 19.9821; 6, 606.5143, 15.7701; 7, 725.6683, 83.2486; 8, 743.4712, 98.1483; 9, 782.3211, 152.9254; 10, 1007.1645, 4.9158; 11, 1128.3325, 103.7911; 12, 1195.6226, 25.8826; 13, 1326.8827, 0.4932; 14, 1778.0997, 144.3793; 15, 1938.8073, 1880.9030; 16, 1988.9472, 183.1079; 17, 3276.7648, 3.4781; 18, 3575.4577, 339.4795;

\section{Calculation summary for 1,3 Migration of H TS2 iZ (acetonitrile)}

Geometry optimisation and frequency calculation: Program version $=$ x86-Linux-G03RevB.01. Method and basis set = RB3LYP/6-31G(d). Calculated Energy = -245.9758603 (hartree), -1028.6710477746 (kJ mol $\left.{ }^{-1}\right)$. Higher level single-point calculation: Program = x86-Linux-G03RevB.01. Method and basis set = RB3LYP/6- 
311++G(3df,2p). Calculated Energy = -246.0762234 (hartree), -1029.0907662588 (kJ mol $\left.{ }^{-1}\right)$. Structure definition (3D Coordinates: Atom, X coord/Å, Y coord/Å, Z coord/Å): C, 0.8879540841, -0.0639890231, 0.1981801293; C, -0.2881681626, -0.0159914739, -0.9664096224; N, -0.8554113098, -0.0181966798, 2.05454099; C, 0.2285735569, 0.0214802895, 1.0108962021; O, 0.2408887444, 0.0630905945, 2.2069485874; H, 1.9315201748, -0.1411083858, -0.4783516838; H, -1.8548770997, 0.0393928951, -2.2080107281; H, 0.986030733, 0.0753687389, 0.3347219399. Harmonic Frequencies (Number, Frequency $\left(\mathrm{cm}^{-1}\right)$, IR Intensity $\left(\mathrm{km} \mathrm{mol}^{-1}\right)$ ): 1, -1285.2, 3507.0311; 2, 151.7198, 170.4872; 3, 261.2395, 5.7270; 4, 270.0457, 12.5645; 5, 580.0648, 30.2877; 6, 613.1183, 39.0246; 7, 665.6912, 176.1952; 8, 724.1633, 94.6988; 9, 909.4478, 106.8762; 10, 1003.329, 4.8375; 11, 1145.1209, 5.5121; 12, 1195.2822, 24.1586; 13, 1326.9812, 0.1344; 14, 1753.4762, 81.8794; 15, 1925.5376, 1900.5892; 16, 1973.9033, 81.3216; 17, 3279.0638, 3.5539; 18, 3658.1983, 419.2171;

\section{Calculation summary for 1,3 Migration of H 1 sE iE (acetonitrile)}

Geometry optimisation and frequency calculation: Program version = x86-Linux-G03RevB.01. Method and basis set = RB3LYP/6-31G(d). Calculated Energy = -246.0567144 (hartree), -1029.0091796208 (kJ mol $\left.{ }^{-1}\right)$. Higher level single-point calculation: Program = x86-Linux-G03RevB.01. Method and basis set = RB3LYP/6311++G(3df,2p). Calculated Energy = -246.1558332 (hartree), -1029.4236944424 (kJ mol $\left.{ }^{-1}\right)$. Structure definition (3D Coordinates: Atom, X coord/A, Y coord/Å, Z coord/Å): C, 0.515289277, 0.0631113819, 0.1519486581; C, 0.5169236101, -0.1591789153, 1.1559662343; O, 0.5080037385, -0.3552990807, 2.3064325541; C, -0.7243843693, 0.104879142, -0.9269749349; N, -0.8353722186, 0.3117299469, 2.1879431313; H, -1.6461915895, -0.0594680863, -0.356540194; H, 1.4858177222, 0.2146456841, 0.6279866961; H, 0.0969783832, 0.4522357675, -2.6135874709. Harmonic Frequencies (Number, Frequency $\left(\mathrm{cm}^{-1}\right)$, IR Intensity $\left.\left(\mathrm{km} \mathrm{mol}^{-1}\right)\right): 1,136.2188,0.5369 ; 2,160.011,15.0211 ; 3,460.1755,7.2115 ; 4,533.2364$, 18.7968; 5, 594.8992, 28.2712; 6, 617.7994, 16.1255; 7, 834.5052, 82.1223; 8, 1057.7363, 31.0268; 9, 1107.0216, 81.0984; 10, 1131.1132, 4.7741; 11, 1266.3375, 372.0897; 12, 1378.6414, 15.2144; 13, 1445.9593, 29.4853; 14, 1662.3794, 550.2223; 15, 2184.4462, 1611.4226; 16, 3072.0628, 21.2649; 17, 3116.6826, 45.0701; 18, 3247.4584, 21.2873;

\section{Calculation summary for 1,3 Migration of H 1 sE iZ (acetonitrile)}

Geometry optimisation and frequency calculation: Program version = x86-Linux-G03RevB.01. Method and basis set = RB3LYP/6-31G(d). Calculated Energy = -246.0560701 (hartree), -1029.0064851582 (kJ mol $\left.{ }^{-1}\right)$. Higher level single-point calculation: Program = x86-Linux-G03RevB.01. Method and basis set = RB3LYP/6311++G(3df,2p). Calculated Energy = -246.1562098 (hartree), -1029.4252693836 (kJ mol $\left.{ }^{-1}\right)$. Structure definition (3D Coordinates: Atom, X coord/A, Y coord/Å, Z coord/Å): C, 0.521656955, -0.0400893672, 0.1757046403; C, -0.736055911, 0.0137719805, -0.9115631275; N, -0.752514962, -0.031567374, 2.194347105; C, 0.5735308692, 0.005071955, 1.1484869359; H, -1.6426842846, 0.0942166545, 0.2947826692; O, 0.6265175741, 0.0444764991, 2.3143123051; H, 1.4722834865, -0.1205864304, 0.7049766192; H, -1.7289265393, 0.0190019913, -2.5216244261. Harmonic Frequencies (Number, Frequency $\left(\mathrm{cm}^{-1}\right)$, IR Intensity $\left.\left(\mathrm{km} \mathrm{mol}^{-1}\right)\right): 1$, 145.8349, 16.3305; 2, 166.1425, 15.6038; 3, 464.1333, 23.4407; 4, 528.0622, 17.2974; 5, 598.3705, 153.1514; 6, 619.0802, 15.2951; 7, 803.0707, 30.2024; 8, 1039.6946, 186.3142; 9, 1086.9626, 7.9565; 10, 1114.2314, 3.8050; 11, 1277.6652, 27.6959; 12, 1370.0658, 55.4141; 13 , 1454.8338, 55.5897; 14, 1668.4827, 482.2132; 15, 2186.8497, 1599.0887; 16, 3027.1689, 25.3503; 17, 3123.1075, 53.1936; 18, 3291.8968, 49.1034;

\section{Calculation summary for 1,3 Migration of $\mathrm{H} 1 \mathrm{sZ}$ iE (acetonitrile)}

Geometry optimisation and frequency calculation: Program version $=$ x86-Linux-G03RevB.01. Method and 
basis set = RB3LYP/6-31G(d). Calculated Energy = -246.0527281 (hartree), -1028.9925089142 (kJ mol $\left.{ }^{-1}\right)$. Higher level single-point calculation: Program = x86-Linux-G03RevB.01. Method and basis set = RB3LYP/6-

311++G(3df,2p). Calculated Energy = -246.1515688 (hartree), -1029.4058607216 (kJ mol $\left.{ }^{-1}\right)$. Structure definition (3D Coordinates: Atom, X coord/Å, Y coord/Å, Z coord/Å): C, 0.7039605043, 0.037521937, 0.5512962287; C, 0.8180821802, 0.1178907627, 0.7676888607; H, 1.6370846531, 0.0964243274, 1.1133411404; O, 0.9104949094, 0.1880360458, 1.9284657783; C, -0.5674956707, -0.1200702596, 1.2674231478; N, -1.7714648552, -0.2122720123, -0.83569199; H, -0.4266051609, -0.1623739251, 2.3538236871; H, -1.8214668638, -0.1644893231, 0.1954656259. Harmonic Frequencies (Number, Frequency $\left(\mathrm{cm}^{-1}\right)$, IR Intensity ( $\left.\mathrm{km} \mathrm{mol}^{-1}\right)$ ): 1, 129.6363, 6.5918; 2, 144.2087, 8.9708; 3, 468.4061, 4.8790; 4, 533.6208, 1.7260; 5, 562.7271, 55.9946; 6, 738.9553, 54.8383; 7, 824.5498, 85.2457; 8, 933.5358, 29.1011; 9, 1109.4507, 65.8277; 10, 1134.7423, 1.1029; 11, 1267.4486, 165.5133; 12, 1410.6198, 277.9611; 13, 1438.5252, 4.6932; 14, 1662.62, 301.8678; 15, 2181.2814, 1284.7915; 16, 3075.9097, 29.6973; 17, 3122.1669, 59.0302; 18, 3265.6459, 6.5170;

\section{Calculation summary for 1,3 Migration of $\mathrm{H} 1 \mathrm{sZ}$ iZ (acetonitrile)}

Geometry optimisation and frequency calculation: Program version = x86-Linux-G03RevB.01. Method and basis set = RB3LYP/6-31G(d). Calculated Energy = -246.0546777 (hartree), $-1029.0006621414\left(\mathrm{~kJ} \mathrm{~mol}^{-1}\right)$. Higher level single-point calculation: Program = x86-Linux-G03RevB.01. Method and basis set = RB3LYP/6311++G(3df,2p). Calculated Energy = -246.1542526 (hartree), -1029.4170843732 (kJ mol $\left.{ }^{-1}\right)$. Structure definition (3D Coordinates: Atom, X coord/Å, Y coord/A, Z coord/Å): C, 0.7407005708, 0.0643925673, 0.5447084848; C, 0.7971985149, 0.0572226924, 0.7812800224; H, 1.6865191902, 0.1452065157, 1.0792885987; O, 0.8450833854, 0.0506099632, 1.9467334698; C, -0.5314404845, -0.0316042164, 1.2589497464; N, -1.6645061294, -0.1278822126, -0.6633219597; H, -0.4171461583, -0.0127318581, 2.3518121277; H, -2.4172488168, -0.1822451342, -1.3652440616. Harmonic Frequencies (Number, Frequency $\left(\mathrm{cm}^{-1}\right)$, IR Intensity $\left.\left(\mathrm{km} \mathrm{mol}^{-1}\right)\right): 1,133.9009,0.4594 ; 2,170.2348,35.6314 ; 3,447.2659,23.4490 ; 4$, 539.7523, 2.4016; 5, 563.5239, 142.8964; 6, 747.8901, 59.2335; 7, 790.5333, 38.6443; 8, 961.2168, 37.9130; 9, 1088.1375, 12.1230; 10, 1128.0303, 1.9216; 11, 1220.8916, 86.5902; 12, 1414.441, 121.4235; 13, 1449.8027, 123.2539; 14, 1667.1447, 269.5767; 15, 2182.142, 1326.0533; 16, 3036.5477, 38.3319; 17, 3141.5266, 58.6701; 18, 3296.1556, 46.2727;

\section{1,3 Migration of $\mathrm{NH}_{2}$}

\section{Calculation summary for 1,3 Migration of $\mathrm{NH}_{2} 3 \mathrm{sE}$ (vacuum)}

Geometry optimisation and frequency calculation: Program version = x86-Linux-G98RevA.7. Method and basis set $=$ RB3LYP/6-31G(d). Calculated Energy = -301.4154443 (hartree), -1260.5193880626 $\left(\mathrm{kJ} \mathrm{mol}^{-1}\right)$. Higher level single-point calculation: Program = x86-Linux-G98RevA.7. Method and basis set = RB3L YP/6$311+G(3 d f, 2 p)$. Calculated Energy = -301.5412555 (hartree), -1261.045530501 (kJ mol $\left.{ }^{-1}\right)$. Structure definition (3D Coordinates: Atom, X coord/A, Y coord/Å, Z coord/Å): C, -0.597157, 0.0803190682, -0.6584548864; C, 0.597157, 0.0803190682, 0.8315751136; N, 0.640144, 0.0803190682, 1.4427381136; C, 0.480315, 0.1045919318, -1.3989018864; N, 1.513667, -0.1509669318, -2.0558448864; O, -1.64138, 0.0443290682, 1.4651781136; H, -1.56606, 0.1818450682, -1.1342068864; H, 0.616746, 0.2420620682, 2.4410701136; H, 1.440716, 0.4572550682, 0.9530341136; H, 1.846955, -1.0775369318, -2.3348848864. Harmonic Frequencies (Number, Frequency $\left(\mathrm{cm}^{-1}\right)$, IR Intensity $\left.\left(\mathrm{km} \mathrm{mol}^{-1}\right)\right)$ : 1, 83.9873, 10.0415; 2, 146.969, 2.5441; 3, 404.3877, 111.4404; 4, 411.7576, 23.3317; 5, 457.5269, 20.0644; 6, 493.4942, 130.3411; 7, 508.7155, 7.8059; 8, 555.8632, 22.5488; 9, 629.0694, 32.9426; 10, 773.4616, 61.5850; 11, 874.059, 62.7146; 12, 902.6062, 11.6811; 
13, 1007.475, 331.9201; 14, 1114.1008, 21.4338; 15, 1142.0467, 6.1887; 16, 1346.0208, 129.5351; 17, 1399.7684, 99.1892; 18, 1653.4308, 102.8031; 19, 1795.0114, 403.7287; 20, 2127.2195, 453.5817; 21, 3231.5121, 6.0302; 22, 3453.8476, 16.2825; 23, 3569.5054, 26.5982; 24, 3687.0558, 27.5610;

\section{Calculation summary for $\mathbf{1 , 3}$ Migration of $\mathrm{NH}_{2} 3 \mathrm{sZ}$ (vacuum)}

Geometry optimisation and frequency calculation: Program version $=$ x86-Linux-G98RevA.7. Method and basis set $=$ RB3LYP/6-31G(d). Calculated Energy = -301.4152628 (hartree), $-1260.5186290296\left(\mathrm{~kJ} \mathrm{~mol}^{-1}\right)$. Higher level single-point calculation: Program $=$ x86-Linux-G98RevA.7. Method and basis set $=$ RB3LYP/6$311+G(3 d f, 2 p)$. Calculated Energy $=-301.5413059$ (hartree), $-1261.0457412738\left(\mathrm{~kJ} \mathrm{~mol}^{-1}\right)$. Structure definition (3D Coordinates: Atom, X coord/A, Y coord/A, Z coord/ $\AA$ ): C, 0.8205745804, 0.0220729204, 0.0524982696; C, 0.8205745804, 0.0220729204, 1.2718177304; H, 1.7750721911, 0.0220729204, 0.5705756386; N, 0.8221489762, 0.1518596984, 2.4837500414; C, -0.4644293974, -0.0307512584, 0.7934069882; O, -1.5547221229, -0.1575645639, -0.2522128693; N, -0.3268393027, 0.0288823296, 2.1651165548; H, -1.1880315111, 0.1599802564, -2.6789733397; H, 0.5057044919, 0.4262818071, 2.5770963595; H, 0.8175455166, -0.6933801639, 3.05843905. Harmonic Frequencies (Number, Frequency

$\left(\mathrm{cm}^{-1}\right)$, IR Intensity $\left.\left(\mathrm{km} \mathrm{mol}^{-1}\right)\right): 1,91.5672,10.0181 ; 2,145.6582,3.2506 ; 3,363.7002,200.7728 ; 4$, 397.1916, 2.3733; 5, 444.6292, 139.7088; 6, 474.2637, 4.0743; 7, 490.6619, 23.5002; 8, 575.5791, 28.6495; 9, 671.6901, 28.2592; 10, 770.0681, 54.5442; 11, 853.0873, 56.8482; 12, 907.1685, 8.2319; 13, 977.3283, 366.5054; 14, 1101.7586, 16.4180; 15, 1160.1773, 6.0182; 16, 1292.1141, 168.7140; 17, 1460.3887, 180.1501; 18, 1648.6897, 129.7317; 19, 1781.772, 237.0805; 20, 2147.3005, 521.3091; 21, 3200.7408, 1.1872; 22, 3476.0574, 22.3933; 23, 3576.0417, 35.7997; 24, 3695.7935, 26.7423;

\section{Calculation summary for 1,3 Migration of $\mathrm{NH}_{2}$ TS2 iE (vacuum)}

Geometry optimisation and frequency calculation: Program version = SGI64-G98RevA.7. Method and basis set = RB3LYP/6-31G(d). Calculated Energy = -301.367619 (hartree), -1260.319382658 $\left(\mathrm{kJ} \mathrm{mol}^{-1}\right)$. Higher level single-point calculation: Program = SGI64-G98RevA.7. Method and basis set $=$ RB3LYP/6-311+G(3df,2p).

Calculated Energy = -301.4910278 (hartree), $-1260.8354782596\left(\mathrm{~kJ} \mathrm{~mol}^{-1}\right)$. Structure definition (3D Coordinates: Atom, X coord/Å, Y coord/Å, Z coord/Å): C, 0.7742289545, 0.0537229545, -0.7008321591; C, 0.7742289545, 0.0537229545, 0.7152858409; O, 1.5705169545, 0.0537229545, 1.6256158409; C, 0.5651900455, -0.0529380455, -1.0305441591; N, -1.5875970455, -0.1305170455, -1.7064561591; N, 0.7386900455, 0.0505279545, 0.8997628409; H, 1.5989439545, 0.1552629545, -1.3932951591; H, 1.1032640455, 0.9413289545, 1.2388048409; H, -1.0860210455, -0.6999140455, 1.5014358409; H, 1.5893920455, -0.5935850455, -2.6084761591. Harmonic Frequencies (Number, Frequency $\left(\mathrm{cm}^{-1}\right)$, IR Intensity ( $\left.\mathrm{km} \mathrm{mol}^{-1}\right)$ ): 1, -512.2108, 39.8688; 2, 133.1165, 11.0394; 3, 359.4034, 10.4495; 4, 422.3294, 86.4982; 5, 457.0213, 125.3374; 6, 510.4628, 32.1951; 7, 563.9675, 30.9402; 8, 614.7322, 23.6795; 9, 679.1544, 42.8877; 10, 759.9931, 13.2980; 11, 824.3522, 145.8995; 12, 866.5037, 142.9365; 13, 950.2762, 62.5863; 14, 1061.4132, 0.3069; 15, 1085.5431, 3.8154; 16, 1230.1327, 69.3108; 17, 1336.8335, 14.5052; 18, 1628.4451, 32.7923; 19, 1868.539, 1127.0800; 20, 2000.3502, 407.4426; 21, 3267.8552, 0.0282; 22, 3450.4605, 14.1440; 23, 3556.3112, 22.4066; 24, 3590.6662, 85.9867;

\section{Calculation summary for $\mathbf{1 , 3}$ Migration of $\mathrm{NH}_{2}$ TS2 iZ (vacuum)}

Geometry optimisation and frequency calculation: Program version = SGI64-G98RevA.7. Method and basis set = RB3LYP/6-31G(d). Calculated Energy = -301.3720548 (hartree), $-1260.3379331736\left(\mathrm{~kJ} \mathrm{~mol}^{-1}\right)$. Higher level single-point calculation: Program = SGI64-G98RevA.7. Method and basis set $=$ RB3LYP/6- 
311+G(3df,2p). Calculated Energy = -301.4952295 (hartree), -1260.853049769 (kJ mol $\left.{ }^{-1}\right)$. Structure definition (3D Coordinates: Atom, X coord/Å, Y coord/Å, Z coord/Å): C, 0.8864725624, 0.0607792389, -0.5678947214; C, 0.8269787743, -0.0224768737, 0.8163615862; N, 1.3758173724, 0.0075369214, 1.9170379897; C, 0.4705959748, 0.0406849626, -0.9466051156; N, -1.0512081777, -0.1071677351, 0.4777944709; O, 1.1550525373, 0.0947262288, -1.9413834067; H, 1.7787213977, 0.1030029786, -1.1758504334; H, 1.5372361712, -0.9952831453, 0.6143557939; H, -1.6820578309, 0.6548738683, 0.7368312469; H, 0.9515963692, -0.2969118025, 2.7807329272. Harmonic Frequencies (Number, Frequency (cm ${ }^{-1}$ ), IR Intensity $\left(\mathrm{km} \mathrm{mol}^{-1}\right)$ ): 1, -402.4325, 128.5382; 2, 132.3805, 12.0036; 3, 318.4707, 104.5532; 4, 376.0341, 40.1028; 5, 435.4078, 40.3299; 6, 508.0631, 1.5937; 7, 584.7795, 1.8608; 8, 620.2848, 1.4030; 9, 688.738, 33.1100; 10, 761.9569, 31.0803; 11, 816.6287, 91.1573; 12, 846.5086, 321.7563; 13, 968.4864, 39.2259; 14, 1055.921, 8.7014; 15, 1075.8628, 3.8429; 16, 1229.165, 80.8288; 17, 1340.6064, 16.5816; 18, 1623.893, 28.0537; 19, 1876.2222, 1205.8883; 20, 1998.8488, 275.9577; 21, 3281.7501, 0.6744; 22, 3443.3367, 11.6545; 23, 3546.6514, 17.5404; 24, 3658.2256, 108.7858;

\section{Calculation summary for 1,3 Migration of $\mathrm{NH}_{2} 1 \mathrm{sE}$ iE (vacuum)}

Geometry optimisation and frequency calculation: Program version = SGI64-G98RevA.7. Method and basis set = RB3LYP/6-31G(d). Calculated Energy = -301.4096516 (hartree), -1260.4951629912 (kJ mol ${ }^{-1}$ ). Higher level single-point calculation: Program = SGI64-G98RevA.7. Method and basis set = RB3LYP/6-

311+G(3df,2p). Calculated Energy = -301.5353379 (hartree), $-1261.0207830978\left(\mathrm{~kJ} \mathrm{~mol}^{-1}\right)$. Structure definition (3D Coordinates: Atom, X coord/A, Y coord/A, Z coord/Å): C, 0.8818126607, -0.0344414603, 0.1247278606; C, 0.8820820349, -0.0337920862, 1.2004486119; O, 0.8838358622, -0.0419165596, 2.3681515447; C, -0.3691533984, 0.0528231221, -0.9132140205; N, -0.4668084008, 0.4612289876, 2.123714903; N, -1.5213622882, -0.303287266, -0.2089839561; H, 1.8442280159, -0.0335865402, 0.6242313695; H, -2.3392235352, -0.2818992625, -0.8100877378; H, -1.4556252036, -1.1703112629, 0.3132787033; H, 0.428680865, 0.8080000367, -2.4703203243. Harmonic Frequencies (Number, Frequency $\left(\mathrm{cm}^{-1}\right)$, IR Intensity $\left.\left(\mathrm{km} \mathrm{mol}^{-1}\right)\right):$ 1, 99.4196, 4.8033; 2, 146.8427, 2.5336; 3, 386.6448, 18.7986; 4, 422.2775, 19.2486; 5, 490.4831, 15.6944; 6, 518.3352, 16.5227; 7, 539.6067, 18.4031; 8, 632.3814, 84.0974; 9, 653.0141, 30.7602; 10, 776.862, 230.4202; 11, 850.6851, 22.9362; 12, 915.3761, 9.8988; 13, 1124.2326, 39.2149; 14, 1150.1036, 30.6794; 15, 1181.1145, 59.5233; 16, 1380.2232, 14.1421; 17, 1424.4518, 204.0879; 18, 1654.2876, 97.0316; 19, 1726.3636, 246.1284; 20, 2223.1583, 745.2336; 21, 3227.2269, 7.2038; 22, 3483.7355, 1.3992; 23, 3528.2618, 14.6242; 24, 3631.5201, 14.6560;

\section{Calculation summary for 1,3 Migration of $\mathrm{NH}_{2} 1 \mathrm{sE}$ iZ (vacuum)}

Geometry optimisation and frequency calculation: Program version = SGI64-G98RevA.7. Method and basis set = RB3LYP/6-31G(d). Calculated Energy = -301.4065441 (hartree), -1260.4821674262 $\left(\mathrm{kJ} \mathrm{mol}^{-1}\right)$. Higher level single-point calculation: Program = SGI64-G98RevA.7. Method and basis set = RB3LYP/6-

311+G(3df,2p). Calculated Energy = -301.5327799 (hartree), -1261.0100855418 (kJ mol $\left.{ }^{-1}\right)$. Structure definition (3D Coordinates: Atom, X coord/Å, Y coord/A, Z coord/Å): C, 0.8618996136, -0.1110558636, 0.1479470909; C, -0.4049553864, -0.0423568636, -0.8999360909; N, -0.3444983864, 0.1185871364, 2.1704100909; C, 0.9532976136, 0.0571201364, 1.1609989091; N, -1.5613523864, -0.0998218636, 0.1101740909; O, 1.0419966136, 0.1846181364, 2.3199419091; H, 1.7821256136, -0.2118878636, 0.7116840909; H, -2.4228153864, -0.1820888636, -0.6380350909; H, -1.5340153864, -0.7591038636, 0.6603099091; H, -1.2817633864, 0.1225341364, -2.5847310909. Harmonic Frequencies (Number, Frequency $\left(\mathrm{cm}^{-1}\right)$, IR Intensity $\left.\left(\mathrm{km} \mathrm{mol}^{-1}\right)\right): 1,91.2603,4.2063 ; 2,143.5409,3.6174 ; 3,338.7737,17.9571 ; 4,425.5015$, 14.6704; 5, 494.267, 19.5220; 6, 520.9635, 19.9498; 7, 526.7617, 53.0195; 8, 600.3041, 136.4349; 9, 655.7396, 
19.5896; 10, 724.9363, 66.8569; 11, 842.1299, 101.5867; 12, 902.582, 11.2913; 13, 1108.1831, 65.7879; 14, 1139.4951, 3.6542; 15, 1151.6457, 67.2935; 16, 1366.4903, 19.6298; 17, 1447.2781, 108.1764; 18, 1673.6513, 98.5178; 19, 1721.9228, 254.3168; 20, 2223.4667, 735.8879; 21, 3237.2734, 17.5972; 22, 3430.4531, 7.1486; 23, 3532.6507, 10.1396; 24, 3634.2245, 8.7150;

\section{Calculation summary for 1,3 Migration of $\mathrm{NH}_{2} 1 \mathrm{sZ} \mathrm{iE} \mathrm{(vacuum)}$}

Geometry optimisation and frequency calculation: Program version = SGI-G98RevA.6. Method and basis set = RB3LYP/6-31G(d). Calculated Energy = -301.4074136 (hartree), $-1260.4858036752\left(\mathrm{~kJ} \mathrm{~mol}^{-1}\right)$. Higher level single-point calculation: Program = SGI-G98RevA.6. Method and basis set = RB3LYP/6-311+G(3df,2p).

Calculated Energy = -301.5332716 (hartree), $-1261.0121418312\left(\mathrm{~kJ} \mathrm{~mol}^{-1}\right)$. Structure definition (3D Coordinates: Atom, X coord/Å, Y coord/Å, Z coord/Å): C, 0.7993857116, -0.1455427094, -0.038933832; C, 0.8843060585, 0.090343741, 1.25975694; H, 1.7258383388, -0.362311136, -0.5621050261; O, 0.9554161253, $0.3054132735,2.405095982$; C, - $0.4943337629,-0.1178387775,-0.7698145941 ; \mathrm{N},-1.6751304589,-$ 0.3007473633, -0.3017794158; N, -0.3272730904, 0.091793298, -2.1352973127; H, -1.2145330636, 0.2337073189, -2.606139552; H, 0.3747771574, 0.7734865462, -2.3968211386; H, -1.6487346335, -

0.587283985, 0.6777838776. Harmonic Frequencies (Number, Frequency $\left(\mathrm{cm}^{-1}\right)$, IR Intensity $\left(\mathrm{km} \mathrm{mol}^{-1}\right)$ ): 1 , 78.4107, 1.8024; 2, 139.235, 3.8123; 3, 395.5432, 23.3993; 4, 425.5508, 35.2957; 5, 493.3052, 15.2402; 6, 503.6388, 44.7038; 7, 542.6583, 18.3885; 8, 634.0121, 154.8980; 9, 655.89, 29.0086; 10, 745.8034, 179.7767; 11, 833.2921, 22.9727; 12, 910.7262, 12.8277; 13, 1117.9062, 59.6939; 14, 1134.2561, 3.9216; 15, 1186.0446, 66.2906; 16, 1344.8061, 25.8924; 17, 1462.525, 189.8667; 18, 1649.3399, 87.4709; 19, 1714.5109, 183.2255; 20, 2228.7269, 697.9590; 21, 3208.5643, 9.3396; 22, 3479.961, 1.7233; 23, 3540.8973, 18.4730; 24, 3649.2268, 16.4336;

\section{Calculation summary for $\mathbf{1 , 3}$ Migration of $\mathrm{NH}_{2} 1 \mathrm{sZ} \mathrm{iZ} \mathrm{(vacuum)}$}

Geometry optimisation and frequency calculation: Program version $=$ x86-Linux-G98RevA.7. Method and basis set $=$ RB3LYP/6-31G(d). Calculated Energy $=-301.4073665$ (hartree), $-1260.485606703\left(\mathrm{~kJ} \mathrm{~mol}^{-1}\right)$. Higher level single-point calculation: Program $=$ x86-Linux-G98RevA.7. Method and basis set $=$ RB3LYP/6$311+G(3 d f, 2 p)$. Calculated Energy $=-301.5337557$ (hartree), $-1261.0141663374\left(\mathrm{~kJ} \mathrm{~mol}^{-1}\right)$. Structure definition (3D Coordinates: Atom, X coord/Å, Y coord/Å, Z coord/Å): C, 0.8255278182, 0.0318736818, 0.0384440909; C, 0.8255278182, 0.0318736818, 1.2883309091; H, 1.7889018182, 0.0318736818, 0.5360620909; O, 0.8624798182, 0.0463856818, 2.4519619091; C, -0.4482281818, -0.0387383182, 0.7747990909; N, -1.5426621818, -0.2296273182, -0.1290720909; N, -0.2967981818, 0.0594986818, 2.1589300909; H, -1.1527581818, 0.2396846818, -2.6696780909; H, 0.4753098182, 0.6260496818, 2.4888690909; H, -2.3520341818, -0.2278473182, -0.7555970909. Harmonic Frequencies (Number, Frequency $\left(\mathrm{cm}^{-1}\right)$, IR Intensity $\left(\mathrm{km} \mathrm{mol}^{-1}\right)$ ): 1, 79.0443, 3.4560; 2, 146.8911, 0.4653; 3, 351.8175, 28.2620; 4, 403.2373, 10.1992; 5, 473.9708, 22.9600; 6, 510.2046, 53.1712; 7, 543.209, 62.8475; 8, 564.7473, 181.5200; 9, 658.7907, 18.7996; 10, 704.3903, 17.4211; 11, 816.3011, 133.7198; 12, 943.4578, 5.9941; 13, 1103.661, 32.0606; 14, 1147.127, 7.5161; 15, 1153.4684, 53.4184; 16, 1339.7711, 78.9759; 17, 1496.0833, 176.7110; 18, 1672.5959, 98.3085; 19, 1712.1, 172.9281; 20, 2240.6305, 705.7843; 21, 3224.4701, 5.3174; 22, 3445.8399, 6.7334; 23, 3547.9857, 13.7000; 24, 3651.5808, 10.9484;

\section{Calculation summary for 1,3 Migration of $\mathrm{NH}_{2} 3 \mathrm{sE}$ (acetonitrile)}

Geometry optimisation and frequency calculation: Program version $=$ x86-Linux-G03RevB.01. Method and basis set $=$ RB3LYP/6-31G(d). Calculated Energy = -301.4381143 (hartree), $-1260.6141940026\left(\mathrm{~kJ} \mathrm{~mol}^{-1}\right)$. 
Higher level single-point calculation: Program = x86-Linux-G03RevB.01. Method and basis set $=$ RB3LYP/6311++G(3df,2p). Calculated Energy = -301.5658679 (hartree), -1261.1484595578 (kJ mol $\left.{ }^{-1}\right)$. Structure

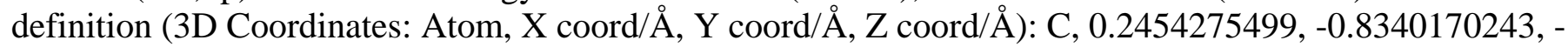
0.072225123; C, -1.020473849, -0.066977601, -0.0037015378; N, -0.9363806325, 1.2951604427, 0.0194834528; C, 1.4577492572, -0.3040822677, -0.008005779; N, 2.5691165323, 0.1965977008, 0.0696227075; O, -2.1054658877, -0.6560213941, 0.0655913229; H, 0.1516032606, -1.9167728594, 0.1292301968; H, -1.811811915, 1.8052105754, -0.0771632874; H, -0.0982859377, 1.7836895662, 0.2842917515; H, 3.0768526457, 0.3641982248, 0.8205240736. Harmonic Frequencies (Number, Frequency $\left(\mathrm{cm}^{-1}\right)$, IR Intensity $\left.\left(\mathrm{km} \mathrm{mol}^{-1}\right)\right): 1,37.4205,15.4800 ; 2,144.4592,9.7865 ; 3,310.6604,337.3163 ; 4$, 420.0709, 36.6044; 5, 451.4309, 17.7560; 6, 490.9914, 100.2702; 7, 511.7797, 4.4748; 8, 539.651, 28.1449; 9, 629.2425, 39.7530; 10, 765.9184, 63.5783; 11, 843.4325, 99.8758; 12, 911.8704, 38.7449; 13, 934.9373, 652.3649; 14, 1104.0007, 17.9862; 15, 1140.2869, 13.3977; 16, 1354.9029, 191.5516; 17, 1410.9397, 225.5967; 18, 1631.6393, 188.7548; 19, 1708.1112, 869.0652; 20, 2114.9196, 880.2749; 21, 3152.8161, 68.2673; 22, 3166.6273, 308.1268; 23, 3456.453, 136.4011; 24, 3564.5656, 115.0122;

\section{Calculation summary for 1,3 Migration of $\mathrm{NH}_{2} 3 \mathrm{sZ}$ (acetonitrile)}

Geometry optimisation and frequency calculation: Program version = x86-Linux-G03RevB.01. Method and basis set = RB3LYP/6-31G(d). Calculated Energy = -301.4399899 (hartree), -1260.6220377618 (kJ mol $\left.{ }^{-1}\right)$. Higher level single-point calculation: Program = x86-Linux-G03RevB.01. Method and basis set = RB3LYP/6311++G(3df,2p). Calculated Energy = -301.5679539 (hartree), -1261.1571832098 (kJ mol $\left.{ }^{-1}\right)$. Structure definition (3D Coordinates: Atom, X coord/Å, Y coord/Å, Z coord/Å): C, 0.81417202, 0.0616261546, 0.0538085089; C, 0.8178693479, 0.0043730585, 1.2708361738; H, 1.7691777725, 0.1318371092, 0.5739361037; N, 0.8214305705, 0.0766199073, 2.4869566959; C, -0.4614332255, -0.002064088, 0.7999624742; O, -1.5655216182, -0.0987851731, -0.246606611; N, -0.3273692181, 0.0336678908, 2.1528400331; H, -1.1693509349, 0.0844581437, -2.7206570649; H, 0.5633205031, 0.2243452033, 2.6035753917; H, 0.8789472837, -0.805984408, 3.0298136636. Harmonic Frequencies (Number, Frequency

$\left(\mathrm{cm}^{-1}\right)$, IR Intensity $\left.\left(\mathrm{km} \mathrm{mol}^{-1}\right)\right): 1,67.1734,16.6206 ; 2,150.6846,10.0397 ; 3,169.2328,366.7568 ; 4$, 409.2934, 6.7892; 5, 444.5568, 84.9156; 6, 479.6292, 10.0627; 7, 493.4588, 43.1093; 8, 589.9246, 15.9698; 9, 674.167, 41.2846; 10, 770.2978, 53.6988; 11, 838.3483, 93.3024; 12, 910.8892, 73.5596; 13, 930.1337, 634.9203; 14, 1096.1713, 4.7202; 15, 1156.9658, 7.4144; 16, 1302.7148, 279.1074; 17, 1459.482, 352.0499; 18, 1616.911, 244.5766; 19, 1699.2014, 470.3868; 20, 2123.7337, 977.4179; 21, 3136.4004, 30.3173; 22, 3174.6079, 332.1369; 23, 3459.2858, 217.4962; 24, 3571.0188, 137.6005;

\section{Calculation summary for 1,3 Migration of $\mathrm{NH}_{2}$ TS2 iE (acetonitrile)}

Geometry optimisation and frequency calculation: Program version = x86-Linux-G03RevB.01. Method and basis set = RB3LYP/6-31G(d). Calculated Energy = -301.3905781 (hartree), -1260.4153976142 (kJ moll $\left.{ }^{-1}\right)$. Higher level single-point calculation: Program = x86-Linux-G03RevB.01. Method and basis set = RB3LYP/6-

$311++G(3 d f, 2 p)$. Calculated Energy = -301.5158495 (hartree), -1260.939282609 $\left(\mathrm{kJ} \mathrm{mol}^{-1}\right)$. Structure definition (3D Coordinates: Atom, X coord/A, Y coord/A, Z coord/Å): C, 0.769668593, 0.0645670502, 0.6964912079; C, 0.7503364127, 0.0517746284, 0.7151754787; O, 1.577044648, 0.0345098896, 1.6167178556; C, -0.5682096463, -0.0470601434, -1.0393887603; N, -1.5835380322, -0.1294340828, 1.7206994914; N, -0.7303906781, 0.0535986967, 0.9226225804; H, 1.5980810913, 0.1727624972, 1.3893208284; H, -1.0893771444, 0.9589665313, 1.251984988; H, -1.0711418498, -0.6893540604, 1.5491530629; H, -1.5671904659, -0.6032955928, -2.6347947534. Harmonic Frequencies (Number, Frequency $\left(\mathrm{cm}^{-1}\right)$, IR Intensity $\left.\left(\mathrm{km} \mathrm{mol}^{-1}\right)\right):$, $-511.5565,76.5429 ; 2,128.537,20.4566 ; 3,344.217,4.4481 ; 4,403.9528$, 
221.1307; 5, 441.8313, 227.4916; 6, 513.776, 35.7961; 7, 568.187, 95.5077; 8, 612.4854, 65.5870; 9, 675.8529, 41.4212; 10, 747.0421, 24.0643; 11, 798.0403, 392.1322; 12, 898.5732, 73.6460; 13, 947.7315, 125.7247; 14 , 1060.6914, 4.1321; 15, 1103.7996, 6.9750; 16, 1239.5394, 98.6939; 17, 1335.7545, 37.3273; 18, 1580.158, 86.6129; 19, 1760.79, 2324.4337; 20, 1977.1704, 753.9780; 21, 3201.0283, 10.2977; 22, 3277.9423, 576.4000; 23, 3314.4777, 102.2676; 24, 3403.6422, 108.0434;

\section{Calculation summary for 1,3 Migration of $\mathrm{NH}_{2}$ TS2' iE (acetonitrile)}

Geometry optimisation and frequency calculation: Program version = x86-Linux-G03RevB.01. Method and basis set = RB3LYP/6-31G(d). Calculated Energy = -301.4095617 (hartree), -1260.4947870294 (kJ mol $\left.{ }^{-1}\right)$. Higher level single-point calculation: Program = x86-Linux-G03RevB.01. Method and basis set $=$ RB3LYP/6311++G(3df,2p). Calculated Energy = -301.5329208 (hartree), -1261.0106747856 (kJ mol $\left.{ }^{-1}\right)$. Structure definition (3D Coordinates: Atom, X coord/Å, Y coord/Å, Z coord/Å): C, -0.0855341512, -1.036185292, 0.00122598; C, -1.1870258907, -0.2263938326, 0.0004706493; O, -2.3433459602, 0.0339115313, 0.0018664582; C, 0.9917385537, -0.0896436093, -0.0007516429; N, 2.2665168754, -0.0765145794, 0.0002761333; N, 0.1927434764, 1.153820502, -0.0000547251; H, -0.0873914823, -2.1214680228, 0.002144191; H, 0.3423346529, 1.7341764626, 0.8356921764; H, 0.3401285876, 1.7337785603, 0.8364296696; H, 2.62180239, -1.0455843062, -0.0006921307. Harmonic Frequencies (Number, Frequency $\left(\mathrm{cm}^{-1}\right)$, IR Intensity $\left(\mathrm{km} \mathrm{mol}^{-1}\right)$ ): 1, -204.9339, 92.5895; 2, 114.6262, 24.4057; 3, 394.198, 87.3011; 4, 395.6884, 35.7116; 5, 484.3378, 31.9106; 6, 575.9216, 20.3810; 7, 634.2203, 4.0982; 8, 666.277, 38.6499; 9, 737.1406, 0.5855; 10, 845.2798, 169.2024; 11, 907.6862, 22.7128; 12, 974.0125, 122.2630; 13, 1055.7696, 54.9697; 14, 1140.0814, 17.1101; 15, 1140.9651, 0.1931; 16, 1327.2126, 35.2624; 17, 1344.7297, 262.8484; 18, 1588.2421, 82.6743; 19, 1725.6276, 1337.4214; 20, 2026.2828, 1312.4630; 21, 3201.2351, 13.2348; 22, 3279.6233, 50.7214; 23, 3324.8927, 133.6162; 24, 3406.4164, 114.7514;

\section{Calculation summary for $\mathbf{1 , 3}$ Migration of $\mathrm{NH}_{2}$ Intermediate $\mathrm{iE}$ (acetonitrile)}

Geometry optimisation and frequency calculation: Program version = DEC-AXP-OSF/1-G03RevB.05. Method and basis set = RB3LYP/6-31G(d). Calculated Energy = -301.4107171 (hartree), $-1260.4996189122\left(\mathrm{~kJ} \mathrm{~mol}^{-1}\right)$. Higher level single-point calculation: Program = DEC-AXP-OSF/1-G03RevB.05. Method and basis set = RB3LYP/6-311++G(3df,2p). Calculated Energy = -301.5344366 (hartree), -1261.0170138612 (kJ mol ${ }^{-1}$ ). Structure definition (3D Coordinates: Atom, X coord/Å, Y coord/Å, Z coord/Å): C, 0.7831097557, 0.0289846091, -0.7444973589; C, 0.9003149881, 0.1028004585, 0.6329496779; O, 1.6264892653, 0.2311477327, 1.5880518949; C, -0.6427565285, -0.1036963256, -0.7767226576; N, -1.6653201267, 0.2211465055, -1.5237536524; N, -0.7649626626, 0.022484543, 0.7238545242; H, 1.5366632519, 0.0538223993, -1.5221160238; H, -1.2530174922, 0.8709132938, 1.0543100478; H, -1.1622774962, 0.8033810278, 1.2006514678; H, -1.3653121518, -0.2929751332, -2.508344722. Harmonic Frequencies (Number, Frequency $\left(\mathrm{cm}^{-1}\right)$, IR Intensity $\left(\mathrm{km} \mathrm{mol}^{-1}\right)$ ): 1, 144.4686, 23.4526; 2, 262.478, 19.7813; 3, 444.7167, 41.9866; 4, 527.3378, 26.6533; 5, 564.4956, 98.0947; 6, 573.8885, 11.7395; 7, 646.4771, 5.1068; 8, 714.5064, 44.8264; 9, 814.9122, 180.8839; 10, 891.5248, 39.0454; 11, 912.8774, 1.3187; 12, 1010.7881, 70.3086; 13, 1092.9964, 0.1218; 14, 1106.6551, 68.0189; 15, 1162.8394, 23.6492; 16, 1296.9285, 37.2992; 17, 1317.5949, 243.4016; 18, 1584.8542, 65.3600; 19, 1721.7725, 2075.5084; 20, 1911.4005, 693.7167; 21, 3230.7235, 11.0976; 22, 3265.3129, 162.7243; 23, 3285.3291, 68.1307; 24, 3339.0413, 156.3616;

\section{Calculation summary for 1,3 Migration of $\mathrm{NH}_{2}$ TS2 iZ (acetonitrile)}

Geometry optimisation and frequency calculation: Program version = x86-Linux-G03RevB.01. Method and 
basis set = RB3LYP/6-31G(d). Calculated Energy = -301.3962119 (hartree), -1260.4389581658 (kJ mol $\left.{ }^{-1}\right)$. Higher level single-point calculation: Program = x86-Linux-G03RevB.01. Method and basis set = RB3LYP/6-

311++G(3df,2p). Calculated Energy = -301.5212927 (hartree), -1260.9620460714 (kJ mol $\left.{ }^{-1}\right)$. Structure definition (3D Coordinates: Atom, X coord/Å, Y coord/A, Z coord/Å): C, 0.8823692325, 0.0359149605, 0.5674478001; C, 0.8525275435, -0.0242591885, 0.8180564977; N, 1.3840310383, 0.0347071889, 1.9213481491; C, -0.4838720852, 0.035477606, -0.9151283934; N, -1.0768108346, -0.1101925907, 0.4558271939; O, -1.1415802265, 0.1186095155, -1.9435321155; H, 1.7682871158, 0.0369451668, 1.1935044632; H, -1.5172684742, -1.0269839006, 0.6073894563; H, -1.7526175161, 0.6281649456, 0.6973574268; H, 0.9775511163, -0.3414047907, 2.7839052779. Harmonic Frequencies (Number, Frequency $\left(\mathrm{cm}^{-1}\right)$, IR Intensity $\left.\left(\mathrm{km} \mathrm{mol}^{-1}\right)\right): 1,-434.1611,251.2948 ; 2,124.7397,21.1862 ; 3,332.2677,95.4793 ; 4$, 399.3359, 46.9997; 5, 413.9237, 217.0969; 6, 510.0707, 9.4900; 7, 589.6486, 0.4252; 8, 621.6598, 21.6707; 9, 676.7064, 46.3775; 10, 735.765, 122.6922; 11, 804.6277, 614.3479; 12, 884.5918, 71.8268; 13, 949.6117, 119.8275; 14, 1061.6132, 30.1561; 15, 1103.3351, 6.9743; 16, 1239.1606, 128.2821; 17, 1339.0995, 35.6543; 18, 1578.476, 84.2405; 19, 1761.3489, 2246.2668; 20, 1987.9497, 636.6952; 21, 3206.9079, 11.6213; 22, 3313.7519, 127.8577; 23, 3346.296, 553.8964; 24, 3402.7795, 105.5956;

\section{Calculation summary for 1,3 Migration of $\mathrm{NH}_{2}$ TS2' iZ (acetonitrile)}

Geometry optimisation and frequency calculation: Program version = x86-Linux-G03RevB.01. Method and basis set = RB3LYP/6-31G(d). Calculated Energy = -301.4078308 (hartree), -1260.4875484056 (kJ mol ${ }^{-1}$ ). Higher level single-point calculation: Program = x86-Linux-G03RevB.01. Method and basis set $=$ RB3LYP/6$311++G(d, p)$. Calculated Energy = -301.5115939 (hartree), -1260.9214856898 $\left(\mathrm{kJ} \mathrm{mol}^{-1}\right)$. Structure definition (3D Coordinates: Atom, X coord/Å, Y coord/Å, Z coord/Å): C, 0.1094826659, -1.0548240534, -0.0005779625; C, -0.9916942249, -0.1412407067, 0.0062947277; N, -2.2579498109, -0.2856666146, 0.0140164318; C, 1.1797525963, -0.2006465671, -0.0074442238; N, -0.1989712155, 1.121373779, 0.0013728837; O, 2.3343601249, 0.0795277248, -0.0147867747; H, 0.1414022952, -2.1389757706, -0.0007803681; H, 0.3412635222, 1.7019236676, -0.8366562042; H, -0.3298494493, 1.7017259885, 0.8413373427; H, 2.7319693617, 0.629422128, 0.01703297. Harmonic Frequencies (Number, Frequency (cm ${ }^{-1}$ ), IR Intensity (km $\left.\mathrm{mol}^{-1}\right)$ ): 1, -200.7898, 93.9955; 2, 130.4132, 20.4450; 3, 396.3444, 104.2105; 4, 428.3519, 36.7009; 5, 502.0706, 11.6529; 6, 581.8593, 9.5090; 7, 631.7222, 0.2330; 8, 678.6092, 115.9594; 9, 748.7932, 38.0420; 10, 798.0944, 40.0475; 11, 897.0405, 24.0492; 12, 971.3706, 150.9107; 13, 1053.6301, 17.1826; 14, 1123.8248, 5.6405; 15, 1137.9961, 304.8821; 16, 1312.6301, 52.8953; 17, 1349.9838, 65.9464; 18, 1581.476, 87.7699; 19, 1736.3196, 1347.1934; 20, 2012.3674, 1241.3190; 21, 3209.4468, 12.5595; 22, 3295.2225, 72.8642; 23, 3311.821, 142.7132; 24, 3395.1275, 123.5563;

\section{Calculation summary for 1,3 Migration of $\mathrm{NH}_{2}$ Intermediate iZ (acetonitrile)}

Geometry optimisation and frequency calculation: Program version = DEC-AXP-OSF/1-G03RevB.05. Method and basis set = RB3LYP/6-31G(d). Calculated Energy = -301.4085465 (hartree), $-1260.490541463\left(\mathrm{~kJ} \mathrm{~mol}^{-1}\right)$. Higher level single-point calculation: Program = DEC-AXP-OSF/1-G03RevB.05. Method and basis set = RB3LYP/6-311++G(3df,2p). Calculated Energy = -301.5329094 (hartree), -1261.0106271108 (kJ mol ${ }^{-1}$ ). Structure definition (3D Coordinates: Atom, X coord/Å, Y coord/Å, Z coord/Å): C, 0.9086671174, 0.096381363, -0.5973540802; C, 0.6381503536, -0.0317595743, 0.7962432802; N, 1.2836490051, 0.0883578362, 1.8894239445; C, -0.4094443966, 0.0628608592, -1.0282351107; N, -0.8720662576, 0.0916170728, 0.5736750991; O, -1.1831361369, 0.0975943398, -1.9533806866; H, 1.8330226948, 0.1897750932, -1.1538541318; H, -1.3225021943, -0.9829543914, 0.8388283001; H, -1.4093347473, 0.6944168115, 0.9751047205; H, 0.6585856622, -0.1870637561, 2.7013487631. Harmonic Frequencies 
(Number, Frequency $\left(\mathrm{cm}^{-1}\right)$, IR Intensity $\left(\mathrm{km} \mathrm{mol}^{-1}\right)$ ): 1, 146.9288, 23.9208; 2, 249.3093, 16.6582; 3, 414.4566, 79.0629; 4, 526.4396, 24.8152; 5, 567.2417, 41.0051; 6, 579.3506, 1.1599; 7, 640.9147, 4.8420; 8, 708.6206, 192.8832; 9, 748.5199, 13.3086; 10, 874.4055, 74.3767; 11, 913.1685, 8.0160; 12, 1014.8631, 8.6930; 13, 1076.4013, 4.2478; 14, 1083.1071, 246.5384; 15, 1151.2616, 209.4595; 16, 1296.8471, 104.4110; 17, 1308.0073, 12.2679; 18, 1579.206, 70.3869; 19, 1737.5903, 2043.2332; 20, 1917.4342, 644.0776; 21, 3231.8644, 10.9388; 22, 3261.4, 144.5274; 23, 3309.6829, 109.5448; 24, 3337.6351, 153.9632;

\section{Calculation summary for 1,3 Migration of $\mathrm{NH}_{2} 1 \mathrm{sE}$ iE (acetonitrile)}

Geometry optimisation and frequency calculation: Program version = x86-Linux-G03RevB.01. Method and basis set = RB3LYP/6-31G(d). Calculated Energy = -301.4266291 (hartree), -1260.5661628962 $\left(\mathrm{kJ} \mathrm{mol}^{-1}\right)$. Higher level single-point calculation: Program = x86-Linux-G03RevB.01. Method and basis set = RB3LYP/6-

311++G(3df,2p). Calculated Energy = -301.5536005 (hartree), -1261.097157291 (kJ mol $\left.{ }^{-1}\right)$. Structure definition (3D Coordinates: Atom, X coord/Å, Y coord/Å, Z coord/Å): C, 0.261916232, -0.8152326535, 0.2072924607; C, 1.4615398847, -0.2915899925, 0.014317151; O, 2.5219662776, 0.1714556417, 0.1558397365; C, -0.986183169, -0.0416208741, 0.0251978302; N, -2.1487496555, -0.5644682715, 0.1572871172; N, -0.8261854525, 1.3392506269, -0.0045921367; H, 0.2183021453, -1.8775807394, 0.445027489; H, -1.7183632997, 1.8339843784, -0.0247190773; H, -0.1975952144, 1.7270884768, 0.6993383821; H, -2.0771657818, -1.5879526168, -0.2206187763. Harmonic Frequencies (Number, Frequency $\left(\mathrm{cm}^{-1}\right)$, IR Intensity $\left.\left(\mathrm{km} \mathrm{mol}^{-1}\right)\right): 1$, 88.2312, 11.2732; 2, 134.5547, 9.4141; 3, 389.874, 27.1788; 4, 436.1473, 43.7682; 5, 487.9475, 23.3342; 6, 514.5245, 18.7075; 7, 534.7789, 43.7401; 8, 618.0091, 255.7957; 9, 650.948, 13.5259; 10, 746.2339, 301.5182; 11, 818.2139, 45.9224; 12, 913.9104, 11.7235; 13, 1120.6485, 34.1639; 14, 1148.0178, 19.0814; 15, 1178.7991, 161.9178; 16, 1378.8708, 36.3908; 17, 1427.6034, 385.4367; 18, 1632.9558, 200.6470; 19, 1686.0031, 487.4926; 20, 2182.6768, 1396.5245; 21, 3137.398, 58.3732; 22, 3333.8273, 39.2867; 23, 3418.2949, 84.1571; 24, 3509.7712, 79.3903;

\section{Calculation summary for $\mathbf{1 , 3}$ Migration of $\mathrm{NH}_{2} 1 \mathrm{sE}$ iZ (acetonitrile)}

Geometry optimisation and frequency calculation: Program version = x86-Linux-G03RevB.01. Method and basis set = RB3LYP/6-31G(d). Calculated Energy = -301.4251685 (hartree), -1260.560054667 (kJ mol $\left.{ }^{-1}\right)$. Higher level single-point calculation: Program $=$ x86-Linux-G03RevB.01. Method and basis set $=$ RB3LYP/6311++G(3df,2p). Calculated Energy = -301.5523288 (hartree), -1261.0918390416 (kJ mol $\left.{ }^{-1}\right)$. Structure definition (3D Coordinates: Atom, X coord/Å, Y coord/Å, Z coord/Å): C, 0.8627279856, -0.1028973602, 0.1461987904; C, -0.4074047021, -0.0417842328, -0.8960131807; N, -0.3498911686, 0.1204879616, 2.1734946336; C, 0.9550994181, 0.0488894429, 1.1637615941; N, -1.5619878136, -0.0931982035, 0.1210291748; O, 1.0493719011, 0.1745775126, 2.3234687973; H, 1.7957698502, -0.2009374017, 0.6987630951; H, -2.4230272182, -0.1951265968, -0.6592857848; H, -1.5443671377, -0.7342226288, 0.6729886662; H, -1.302734037, 0.1173911208, -2.5703212439. Harmonic Frequencies (Number, Frequency $\left(\mathrm{cm}^{-1}\right)$, IR Intensity $\left.\left(\mathrm{km} \mathrm{mol}^{-1}\right)\right): 1,85.015,6.5937 ; 2,131.8093,10.3698 ; 3,363.445,39.2106 ; 4,430.9772$, 38.4363; 5, 494.782, 27.1747; 6, 512.8231, 45.8715; 7, 525.681, 66.4490; 8, 593.9418, 250.7962; 9, 651.2307, 24.4710; 10, 717.6632, 95.4652; 11, 829.5286, 156.1291; 12, 904.0886, 20.3110; 13, 1108.0934, 59.0414; 14, 1137.848, 2.9303; 15, 1172.9733, 164.5849; 16, 1369.1421, 20.4038; 17, 1448.9619, 204.4102; 18, 1645.7447, 230.3356; 19, 1681.3142, 478.7937; 20, 2185.8778, 1379.1028; 21, 3146.0962, 65.3238; 22, 3276.9899, 30.1388; 23, 3413.1526, 83.9549; 24, 3502.9322, 77.6952;

Calculation summary for $\mathbf{1 , 3}$ Migration of $\mathrm{NH}_{2} 1 \mathrm{sZ}$ iE (acetonitrile) 
Geometry optimisation and frequency calculation: Program version = DEC-AXP-OSF/1-G03RevB.05. Method and basis set = RB3LYP/6-31G(d). Calculated Energy = -301.4248042 (hartree), $-1260.5585311644\left(\mathrm{~kJ} \mathrm{~mol}^{-1}\right)$. Higher level single-point calculation: Program = DEC-AXP-OSF/1-G03RevB.05. Method and basis set = RB3LYP/6-311++G(3df,2p). Calculated Energy = -301.5522778 (hartree), -1261.0916257596 (kJ mol ${ }^{-1}$ ).

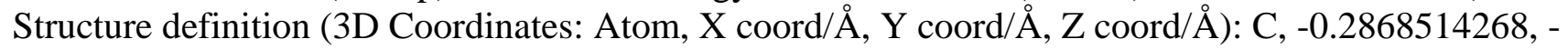
0.7203242442, -0.1967198372; C, -1.5166496442, -0.2712554327, -0.0166447783; H, -0.1940737369, 1.7807405646, -0.4358501672; O, -2.6048156695, 0.1234088806, 0.1442644366; C, 0.9163068026, 0.1321931638, -0.0395107618; N, 0.9754339125, 1.4217733581, -0.0302945308; N, 2.0804292981, 0.6091025324, 0.0543814634; H, 2.8904297312, -0.064275604, 0.3468971223; H, 2.0150854358, 1.5143473383, 0.5158712671; H, 0.059207063, 1.8397157614, -0.2323899788. Harmonic Frequencies

(Number, Frequency $\left(\mathrm{cm}^{-1}\right)$, IR Intensity $\left.\left(\mathrm{km} \mathrm{mol}^{-1}\right)\right): 1,61.9188,3.1061 ; 2,141.139,5.6045 ; 3,397.6275$, 78.5952; 4, 422.0225, 17.1058; 5, 488.9438, 77.3697; 6, 504.7685, 104.0677; 7, 537.1704, 50.0841; 8, 579.4378, 281.0274; 9, 658.9163, 43.9748; 10, 721.5888, 146.7282; 11, 788.5763, 49.0246; 12, 906.4634, 13.0258; 13, 1119.776, 60.3187; 14, 1134.2344, 12.3423; 15, 1187.6516, 181.9238; 16, 1354.1901, 50.1693; 17, 1464.1143, 361.1819; 18, 1625.2575, 150.5193; 19, 1673.6335, 334.3348; 20, 2189.9901, 1280.1518; 21, 3118.0083, 58.1394; 22, 3344.0411, 18.0260; 23, 3438.3679, 117.5498; 24, 3536.8568, 91.1790;

\section{Calculation summary for $\mathbf{1 , 3}$ Migration of $\mathrm{NH}_{2} 1 \mathrm{sZ}$ iZ (acetonitrile)}

Geometry optimisation and frequency calculation: Program version = x86-Linux-G03RevB.01. Method and basis set = RB3LYP/6-31G(d). Calculated Energy = -301.4266521 (hartree), -1260.5662590822 (kJ mol $\left.{ }^{-1}\right)$. Higher level single-point calculation: Program = x86-Linux-G03RevB.01. Method and basis set = RB3LYP/6$311++G(3 d f, 2 p)$. Calculated Energy = -301.5540153 (hartree), -1261.0988919846 (kJ mol $\left.{ }^{-1}\right)$. Structure definition (3D Coordinates: Atom, X coord/A, Y coord/A, Z coord/Å): C, 0.827717184, 0.0592508707, 0.0398767438; C, 0.8385150249, 0.0196412217, 1.282532602; H, 1.7903374107, 0.1415850313, 0.5431332136; O, 0.8658413111, -0.0092849642, 2.4500552142; C, -0.4495499407, -0.018312216, 0.7752163539; N, -1.5520085676, -0.196594144, -0.1235694307; N, -0.3072107943, 0.0523415995, 2.1483005784; H, -1.1598143816, 0.2476079318, -2.6715149634; H, 0.5082174112, 0.5421043296, 2.5124336457; H, -2.3510290057, -0.210729026, -0.7749068522. Harmonic Frequencies (Number, Frequency $\left(\mathrm{cm}^{-1}\right)$, IR Intensity $\left.\left(\mathrm{km} \mathrm{mol}^{-1}\right)\right): 1,62.2145,5.0028 ; 2,132.7408,0.6236 ; 3,381.4979,60.3273 ; 4,404.737$, 23.2246; 5, 472.1644, 116.9059; 6, 502.5984, 325.0369; 7, 530.3532, 39.4777; 8, 542.4758, 42.4470; 9 , 662.5863, 27.0643; 10, 699.4448, 9.9280; 11, 800.7958, 173.1275; 12, 935.7362, 11.3147; 13, 1110.9756, 17.8842; 14, 1137.0121, 10.9877; 15, 1178.3409, 111.3174; 16, 1344.8727, 141.6216; 17, 1489.5383, 326.2643; 18, 1639.3292, 161.9896; 19, 1666.8369, 327.4874; 20, 2192.6211, 1295.7631; 21, 3139.1616, 53.5493; 22, 3292.6806, 19.3665; 23, 3433.2397, 123.7906; 24, 3529.6062, 92.9964;

\section{1,3 Migration of $\mathrm{NMe}_{2}$}

\section{Calculation summary for 1,3 Migration of $\mathrm{NMe}_{2} 3 \mathrm{sE}$ (vacuum)}

Geometry optimisation and frequency calculation: Program version = x86-Linux-G98RevA.7. Method and basis set $=$ RB3LYP/6-31G(d). Calculated Energy = -380.028726 (hartree), -1589.280132132 $\left(\mathrm{kJ} \mathrm{mol}^{-1}\right)$. Higher level single-point calculation: Program = x86-Linux-G98RevA.7. Method and basis set = RB3LYP/6311+G(3df,2p). Calculated Energy = -380.1725286 (hartree), $-1589.8815146052\left(\mathrm{~kJ} \mathrm{~mol}^{-1}\right)$. Structure definition (3D Coordinates: Atom, X coord/Å, Y coord/Å, Z coord/ $\AA$ ): C, 0.127394624, 0.1964411058, 0.9184738864; O, 0.1269429983, 0.1979029778, 2.1466389147; C, 1.4443185267, 0.1953778342, 0.2111188588; N, -1.0374415995, 0.1745311087, 0.1828720588; C, 1.7296387167, -0.3617270106, - 
0.9517579243; N, 2.0172912419, -0.9938567763, -1.9621758945; H, 2.0611830209, -0.4729939641, 2.8418672161; H, 2.2725618263, 0.5392939464, 0.8229697111; C, -1.1395845943, 0.4209702678, 1.2452077671; C, -2.2979542244, 0.0009700917, 0.8917576556; H, -2.8990106359, 0.9200655171, 0.8554480971; H, -2.0808622631, -0.2407774391, 1.9309107956; H, -2.8787927177, -0.8105618221, 0.4352444991; H, -2.0281916611, 1.0339175749, -1.4377461094; H, -1.2334286128, -0.5062543027, 1.8281302221; H, -0.2708287322, 0.9771726068, -1.6011222794. Harmonic Frequencies (Number, Frequency $\left(\mathrm{cm}^{-1}\right)$, IR Intensity $\left.\left(\mathrm{km} \mathrm{mol}^{-1}\right)\right): 1,54.4138,0.2082 ; 2,105.2069,0.2782 ; 3,108.1923,2.8288 ; 4,154.3199$, 4.2943; 5, 192.5638, 0.9926; 6, 215.0286, 4.2327; 7, 316.7604, 8.1796; 8, 405.3571, 5.8916; 9, 439.4128, 27.1815; 10, 448.1064, 30.8554; 11, 480.8375, 6.5013; 12, 543.8975, 25.4527; 13, 696.4473, 4.4955; 14, 722.492, 13.1604; 15, 777.8373, 44.8943; 16, 871.129, 60.1049; 17, 1001.6091, 56.2866; 18, 1013.5121, 273.9894; 19, 1090.3053, 7.4680; 20, 1130.6876, 97.5990; 21, 1141.7651, 1.5372; 22, 1179.0461, 2.9823; 23, 1216.3624, 75.8686; 24, 1294.1545, 50.7556; 25, 1391.0225, 3.5098; 26, 1426.0584, 188.1753; 27, 1453.0838, 10.9279; 28, 1492.4813, 37.0168; 29, 1522.3935, 10.6922; 30, 1523.5392, 15.3388; 31, 1536.1201, 7.2839; 32, 1558.7441, 30.0670; 33, 1740.82, 375.4558; 34, 2123.7537, 457.2001; 35, 3025.4345, 38.2203; 36, 3033.2266, 69.4861; 37, 3078.0124, 56.3090; 38, 3084.9489, 14.4622; 39, 3153.6686, 16.0907; 40, 3194.7796, 1.1851; 41, 3212.0304, 4.3261; 42, 3450.7713, 10.1021;

\section{Calculation summary for 1,3 Migration of $\mathrm{NMe}_{2} 3 \mathrm{sZ}$ (vacuum)}

Geometry optimisation and frequency calculation: Program version = x86-Linux-G98RevA.7. Method and basis set $=$ RB3LYP/6-31G(d). Calculated Energy = -380.0318042 (hartree), -1589.2930051644 $\left(\mathrm{kJ} \mathrm{mol}^{-1}\right)$. Higher level single-point calculation: Program = x86-Linux-G98RevA.7. Method and basis set = RB3LYP/6-

311+G(3df,2p). Calculated Energy = -380.1757322 (hartree), $-1589.8949120604\left(\mathrm{~kJ} \mathrm{~mol}^{-1}\right)$. Structure definition (3D Coordinates: Atom, X coord/Å, Y coord/A, Z coord/Å): C, -0.1548561747, -0.0403158703, 0.3117983575; O, -0.1544542828, -0.0398844741, 1.5429361141; C, 1.1199976283, -0.0409041139, 0.4523948412; N, -1.322924365, -0.0517122131, -0.4222625784; C, 2.2560128964, 0.1339276123, 0.2074188555; N, 3.2968467183, 0.4208586435, 0.773491524; H, 3.8214596987, -0.3325380135, 1.2229410664; H, 1.1827085347, -0.2488344321, -1.51390665; C, -1.3978354274, 0.0004164724, 1.8725438568; C, -2.5846389644, -0.2181694944, 0.2841310411; H, -3.2567604833, 0.6247591492, 0.0770044328; H, -2.3743312832, -0.259439804, 1.3517671974; H, -3.0861590717, -1.1449374525, 0.0275467591; H, -2.3259043021, 0.5060384557, -2.1599854747; H, -1.4028137594, -0.9992982055, 2.3331223129; H, -0.5721012943, 0.5795734465, -2.2897003686. Harmonic Frequencies (Number, Frequency $\left(\mathrm{cm}^{-1}\right)$, IR Intensity $\left.\left(\mathrm{km} \mathrm{mol}^{-1}\right)\right): 1,54.9541,3.2545 ; 2,91.4048,2.6754 ; 3,100.5927,0.3499 ; 4,137.6135$, 1.5693; 5, 154.3422, 2.8035; 6, 212.5552, 4.4463; 7, 316.6781, 6.6330; 8, 396.0602, 3.6041; 9, 440.9907, 1.3050; 10, 449.9696, 59.4214; 11, 499.2135, 11.1675; 12, 525.7683, 27.3648; 13, 638.6014, 8.0106; 14, 756.3707, 35.2501; 15, 850.5502, 51.1335; 16, 870.7791, 5.1288; 17, 977.3675, 397.0921; 18, 994.6341, 28.3033; 19, 1091.3091, 7.1803; 20, 1137.9144, 37.9189; 21, 1142.7566, 218.5609; 22, 1170.9505, 1.7881; 23, 1181.343, 8.6901; 24, 1299.0101, 48.0971; 25, 1386.3831, 44.7466; 26, 1447.8967, 48.6059; 27, 1460.8849, 187.5803; 28, 1496.9844, 53.2534; 29, 1526.8257, 8.5181; 30, 1529.5251, 17.5959; 31, 1535.5719, 13.2960; 32, 1557.9333, 51.9681; 33, 1726.507, 226.0808; 34, 2144.0592, 614.8936; 35, 3015.1428, 55.8991; 36, 3028.5823, 84.6593; 37, 3074.3955, 46.7856; 38, 3094.3465, 28.1975; 39, 3151.7031, 15.5733; 40, 3193.5017, 1.3933; 41, 3227.6697, 3.3716; 42, 3473.5485, 20.2670;

\section{Calculation summary for 1,3 Migration of $\mathrm{NMe}_{2}$ TS2 iE (vacuum)}

Geometry optimisation and frequency calculation: Program version = SGI64-G98RevA.7. Method and basis set = RB3LYP/6-31G(d). Calculated Energy = -379.9923626 (hartree), -1589.1280603932 $\left(\mathrm{kJ} \mathrm{mol}^{-1}\right)$. Higher level single-point calculation: Program = SGI64-G98RevA.7. Method and basis set = RB3LYP/6- 
311+G(3df,2p). Calculated Energy = -380.1361078 (hartree), -1589.7292028196 (kJ mol $\left.{ }^{-1}\right)$. Structure definition (3D Coordinates: Atom, X coord/Å, Y coord/A, Z coord/Å): C, 1.3846728045, 0.1005066462, 0.7902934167; C, 1.3743978275, 0.1018579229, 0.5873670517; N, 1.7677183426, 0.0972769417, 1.7515198861; C, 0.011589119, 0.0688621653, -1.1502048464; N, -0.5751934427, -0.0672231381, 0.2401226471; O, -0.6553694382, 0.126095359, -2.1603231523; H, 2.2620647036, 0.0934407542, 1.4246044941; C, -1.1200482831, -1.3992734659, 0.5222197782; C, -1.4853142517, 1.0159443103, 0.6205339832; H, 2.5255432576, 0.6910497351, 2.069727478; H, -1.6756934966, 0.9614174671, 1.6971263084; H, -1.0177931853, 1.9767911134, 0.3904884382; H, -2.4375892351, 0.9448612953, 0.0790844445; H, -1.3079410104, -1.4914394102, 1.5965831686; H, -2.0555206618, -1.5676503214, 0.0279399677; H, -0.3895724625, -2.1549956032, 0.2228868108. Harmonic Frequencies (Number, Frequency $\left(\mathrm{cm}^{-1}\right)$, IR Intensity $\left.\left(\mathrm{km} \mathrm{mol}^{-1}\right)\right): 1,-445.123,57.7658 ; 2,110.4201,5.8244 ; 3,133.8001,2.7964 ; 4,151.494$, 2.5855; 5, 195.6336, 1.1764; 6, 249.8181, 0.4059; 7, 267.7651, 5.0591; 8, 302.3158, 3.9898; 9, 398.705, 12.7312; 10, 471.2006, 170.1876; 11, 553.722, 36.6328; 12, 578.8869, 29.8431; 13, 650.84, 3.4236; 14, 710.9426, 51.1514; 15, 732.3837, 5.6390; 16, 768.822, 13.9783; 17, 831.0281, 269.9129; 18, 976.94, 102.5562; 19, 1062.3272, 6.4445; 20, 1065.4596, 5.5996; 21, 1112.1557, 1.3283; 22, 1206.6234, 2.3012; 23, 1220.2203, 74.7418; 24, 1222.7933, 29.7073; 25, 1296.5766, 10.4860; 26, 1336.1294, 13.4784; 27, 1451.9292, 0.1568; 28, 1481.2441, 0.6302; 29, 1507.7027, 3.6919; 30, 1516.2932, 5.3640; 31, 1523.7853, 7.2555; 32, 1533.4918, 11.7460; 33, 1846.5861, 919.4222; 34, 2004.3416, 456.1967; 35, 3039.259, 27.3122; 36, 3045.5843, 49.5162; 37, 3115.6018, 9.2345; 38, 3117.3614, 18.6158; 39, 3149.8491, 12.5354; 40, 3152.6804, 13.1622; 41, 3253.3341, 0.5526; 42, 3584.2853, 73.8319;

\section{Calculation summary for 1,3 Migration of $\mathrm{NMe}_{2}$ TS2 iZ (vacuum)}

Geometry optimisation and frequency calculation: Program version = SGI64-G98RevA.7. Method and basis set = RB3LYP/6-31G(d). Calculated Energy = -379.9964955 (hartree), -1589.145344181 (kJ mol ${ }^{-1}$ ). Higher level single-point calculation: Program = SGI64-G98RevA.7. Method and basis set = RB3LYP/6-311+G(3df,2p).

Calculated Energy = -380.13963 (hartree), -1589.74393266 $\left(\mathrm{kJ} \mathrm{mol}^{-1}\right)$. Structure definition (3D Coordinates: Atom, X coord $/ \AA$, Y coord/A, Z coord/Å): C, 1.1085333341, 0.0126602592, -1.1601416973; C, 1.1202535595, 0.0079087847, 0.2533061376; O, 1.9255955821, 0.0048631913, 1.1589023317; C, -0.2352636034, 0.0393248802, -1.4750518375; N, -1.1584542951, -0.2004290185, -2.2736152948; N, -0.3913082981, 0.0207254623, 0.4863819929; H, 1.9322210314, 0.0744928807, -1.8583825119; C, -0.8584977829, 1.2784932041, 1.0810433498; C, -0.8783072351, -1.1638249483, 1.203648593; H, -2.0905081545, 0.1695297058, -2.1408226397; H, -1.9534736769, 1.2969856688, 1.093003279; H, -0.4854978546, 1.3862429391, 2.1079032718; H, -0.4900740818, 2.1155094298, 0.4819573339; H, -1.9731141643, 1.1634891874, 1.2099519146; H, -0.5254091893, -2.0622199412, 0.6912660168; H, -0.5108800471, -

1.1735066491, 2.2377105219. Harmonic Frequencies (Number, Frequency $\left(\mathrm{cm}^{-1}\right)$, IR Intensity $\left(\mathrm{km} \mathrm{mol}^{-1}\right)$ ): 1 , -358.6999, 178.9197; 2, 113.6153, 1.7080; 3, 139.5583, 0.4301; 4, 173.8584, 1.9842; 5, 205.0118, 1.9598; 6, 248.5708, 4.1102; 7, 270.6247, 14.4805; 8, 298.2686, 20.0021; 9, 390.408, 7.0988; 10, 476.6021, 83.1303; 11, 550.508, 25.8318; 12, 595.9721, 21.5266; 13, 649.064, 2.4269; 14, 710.6737, 70.0428; 15, 726.5664, 23.2791; 16, 763.4753, 14.9117; 17, 846.4876, 407.8758; 18, 963.7539, 94.2175; 19, 1060.5849, 6.7294; 20, 1062.9874, 16.9818; 21, 1111.4635, 0.8422; 22, 1208.5529, 14.7015; 23, 1218.6142, 14.0448; 24, 1222.3646, 98.2696; 25, 1287.6626, 6.1053; 26, 1338.6792, 13.9973; 27, 1451.0969, 0.2147; 28, 1480.814, 0.4251; 29, 1508.59, 3.2149; 30, 1518.5043, 5.5607; 31, 1524.2736, 8.4878; 32, 1535.2749, 11.4495; 33, 1850.4736, 963.3795; 34, 1997.6706, 336.2645; 35, 3041.5101, 26.2147; 36, 3048.0871, 46.1005; 37, 3111.6824, 13.2168; 38, 3117.5034, 16.6476; 39, 3146.5929, 13.0135; 40, 3152.8834, 12.2177; 41, 3267.0596, 0.0393; 42, 3621.6819, 63.7708;

Calculation summary for $\mathbf{1 , 3}$ Migration of $\mathrm{NMe}_{2} 1 \mathrm{sE} \mathrm{iE} \mathrm{(vacuum)}$ 
Geometry optimisation and frequency calculation: Program version = SGI64-G98RevA.7. Method and basis set = RB3LYP/6-31G(d). Calculated Energy = -380.0230594 (hartree), -1589.2564344108 $\left(\mathrm{kJ} \mathrm{mol}^{-1}\right)$. Higher level single-point calculation: Program = SGI64-G98RevA.7. Method and basis set = RB3LYP/6-

311+G(3df,2p). Calculated Energy = -380.167172 (hartree), -1589.859113304 ( $\left.\mathrm{kJ} \mathrm{mol}^{-1}\right)$. Structure definition

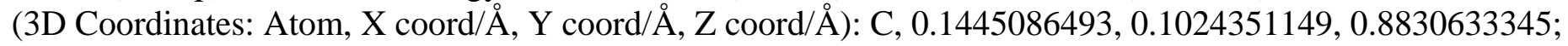
N, 0.0059185899, 0.02325397, 2.1583795241; C, 1.4658400484, 0.1771041219, 0.201142978; N, 0.9762045819, 0.0353614451, 0.0532402423; C, 1.7000717394, -0.3918038131, -0.9704875728; O, 1.9017102267, -0.88324645, -2.0115542296; H, 2.3164876418, 0.5978685825, 0.7265981562; C, 1.1373958733, 0.9824944814, -1.049092239; C, -2.2393663727, -0.2812546222, 0.7122806382; H, 2.6463610316, 0.576783404, 1.2678428881; H, -2.0878844681, -1.0967465184, 1.4197762953; H, 2.9620842979, -0.5831215949, -0.0533123902; H, -1.6476233824, 1.9013802803, -0.7171090851; H, 1.7393972078, 0.5253965053, -1.8420928822; H, -0.1716255214, 1.2658419578, -1.4669547297; H, 0.9148592509, -0.0655906197, 2.614904387. Harmonic Frequencies (Number, Frequency ( $\mathrm{cm}^{-1}$ ), IR Intensity $\left(\mathrm{km} \mathrm{mol}^{-1}\right)$ ): 1, 73.2044, 0.7373; 2, 87.1909, 3.8373; 3, 123.7206, 2.8711; 4, 171.3397, 2.0326; 5, 200.3708, 1.3575; 6, 274.5621, 14.1337; 7, 322.6205, 5.6542; 8, 403.1439, 1.7229; 9, 445.1269, 1.6571; 10, 487.0753, 3.1179; 11, 527.3234, 5.0215; 12, 548.7907, 34.6369; 13, 694.5436, 2.9919; 14, 715.5911, 39.9672; 15 , 765.1722, 3.8709; 16, 837.5948, 67.2066; 17, 997.5977, 7.2284; 18, 1083.1177, 25.6139; 19, 1103.3187, 122.4445; 20, 1136.2171, 4.9970; 21, 1146.6083, 23.9574; 22, 1184.9924, 11.4893; 23, 1243.4602, 7.4245; 24, 1303.7618, 96.9880; 25, 1381.3295, 4.6620; 26, 1423.934, 210.1192; 27, 1466.0493, 1.2499; 28, 1495.5609, 24.9350; 29, 1505.047, 3.8625; 30, 1521.09, 14.4283; 31, 1529.7523, 7.8432; 32, 1553.4553, 26.0311; 33, 1674.6162, 241.6189; 34, 2220.4394, 712.1613; 35, 3001.2609, 63.7764; 36, 3023.3759, 62.2655; 37, 3090.7417, 26.9402; 38, 3096.9568, 39.8966; 39, 3174.3799, 9.7700; 40, 3175.3609, 13.6559; 41, 3220.0778, 6.7920; 42, 3479.1693, 1.3724;

\section{Calculation summary for 1,3 Migration of $\mathrm{NMe}_{2} 1 \mathrm{sE}$ iZ (vacuum)}

Geometry optimisation and frequency calculation: Program version = SGI64-G98RevA.7. Method and basis set = RB3LYP/6-31G(d). Calculated Energy = -380.0198509 (hartree), -1589.2430164638 $\left(\mathrm{kJ} \mathrm{mol}^{-1}\right)$. Higher level single-point calculation: Program = SGI64-G98RevA.7. Method and basis set = RB3LYP/6311+G(3df,2p). Calculated Energy = -380.1639338 (hartree), $-1589.8455711516\left(\mathrm{~kJ} \mathrm{~mol}^{-1}\right)$. Structure definition (3D Coordinates: Atom, X coord/Å, Y coord/Å, Z coord/Å): C, 0.3734032898, 0.8316235462, 0.1269907327; N, 1.0745442978, 1.9073156868, 0.1192831879; C, -1.0820624821, 0.9830655654, 0.3368515056; N, 0.8232876202, -0.4802781835, -0.1329356126; C, -1.9874763619, 0.1417402392, 0.1352725335; O, -2.7998550756, -0.6061102851, -0.5198787385; H, -1.4376475947, 1.9208321927, 0.7491159858; H, 2.0717182667, 1.7044123565, 0.0250413179; C, 2.1715864078, -0.6112577993, 0.6637800799; C, 0.4802232504, -1.5557891817, 0.8004974719; H, 1.2219589494, -1.6381547111, 1.612016752; H, 0.4464473961, -2.5129009172, 0.2683503783; H, -0.494065708, -1.3769237012, 1.2547279373; H, 2.9564212758, -0.4437693604, 0.0943838993; H, 2.2961032618, -1.6255323232, 1.0557124336; H, 2.3190367077, 0.0953620023, -1.4850495372. Harmonic Frequencies (Number, Frequency $\left(\mathrm{cm}^{-1}\right)$, IR Intensity $\left.\left(\mathrm{km} \mathrm{mol}^{-1}\right)\right): 1,66.2206,0.6794 ; 2,100.1119,2.4850 ; 3,118.2976,2.6002 ; 4,169.8627$, 0.3516; 5, 245.1473, 1.7844; 6, 271.3546, 2.7490; 7, 324.1826, 3.8104; 8, 398.6718, 3.3279; 9, 442.5989, 5.6325; 10, 489.0608, 18.6631; 11, 527.8709, 1.2436; 12, 556.8902, 48.1001; 13, 694.1041, 9.8937; 14, 717.5079, 21.1731; 15, 756.06, 6.6220; 16, 851.0795, 53.4929; 17, 985.301, 42.6631; 18, 1083.6891, 49.3605; 19, 1095.3771, 63.9670; 20, 1134.5027, 3.6722; 21, 1141.7286, 12.6464; 22, 1181.5178, 16.1661; 23, 1244.0597, 48.5211; 24, 1304.9707, 21.7824; 25, 1371.7112, 38.4136; 26, 1421.9961, 165.2430; 27, 1466.6198, 2.9040; 28, 1498.5152, 12.8274; 29, 1512.9023, 8.6850; 30, 1525.7371, 13.1235; 31, 1535.2156, 2.8254; 32, 1552.2132, 20.7578; 33, 1675.649, 246.0327; 34, 2221.3086, 716.3975; 35, 2981.6347, 50.9773; 
36, 2998.3719, 82.8513; 37, 3089.4558, 29.5462; 38, 3095.1956, 28.9227; 39, 3142.1956, 14.8447; 40, 3176.4668, 12.4777; 41, 3229.5831, 14.7047; 42, 3473.3646, 2.5466;

\section{Calculation summary for 1,3 Migration of $\mathrm{NMe}_{2} 1 \mathrm{sZ}$ iE (vacuum)}

Geometry optimisation and frequency calculation: Program version = SGI64-G98RevA.7. Method and basis set = RB3LYP/6-31G(d). Calculated Energy = -380.0227095 (hartree), -1589.254971129 (kJ mol $\left.{ }^{-1}\right)$. Higher level single-point calculation: Program = SGI64-G98RevA.7. Method and basis set = RB3LYP/6-311+G(3df,2p).

Calculated Energy = -380.166757 (hartree), -1589.857377774 $\left(\mathrm{kJ} \mathrm{mol}^{-1}\right)$. Structure definition (3D Coordinates: Atom, X coord $/ \AA$, Y coord/A, Z coord $/ \AA$ ): C, -0.1240152182, -0.325157588, 0.3253392482; N, -0.2497251553, -0.5001061311, 1.595876384; C, 1.1668791109, -0.1772757511, -0.407365227; N, -1.2519120916, 0.3328359612, -0.4850722766; C, 2.2114701233, 0.4209654424, 0.1389353048; O, 3.1330598047, 0.9548027718, 0.6199112064; H, 1.3040794992, -0.5840740768, -1.404422438; H, 0.6627449159, 0.642235316, 2.0305462913; C, -2.5254758686, -0.6056971529, 0.1697988884; C, -1.3589448812, 0.5433470662, -1.6438910425; H, -1.7823750782, 1.5257356747, -1.3773569413; H, -2.0139679411, 0.083442301, -2.392451986; H, -0.3814331743, 0.7124590808, -2.0962995729; H, -2.9108681872, 0.2711670981, 0.7114052805; H, -3.2540038762, -0.8984497475, -0.5939245311; H, -2.3966734646, 1.4129646409, 0.8906824627. Harmonic Frequencies (Number, Frequency $\left(\mathrm{cm}^{-1}\right)$, IR Intensity $\left(\mathrm{km} \mathrm{mol}^{-1}\right)$ ): 1 , 59.1039, 0.2161; 2, 93.6482, 2.7776; 3, 129.77, 1.7571; 4, 160.6338, 2.5889; 5, 188.8182, 1.3259; 6, 256.8597, 18.8770; 7, 315.6859, 3.4632; 8, 401.5312, 5.2104; 9, 452.508, 4.7820; 10, 496.9433, 7.6467; 11, 532.9173, 3.1224; 12, 545.5421, 17.7304; 13, 637.0896, 30.8119; 14, 725.312, 10.5346; 15, 826.8879, 40.8576; 16, 855.8246, 24.7923; 17, 972.7066, 3.4611; 18, 1084.9313, 54.4288; 19, 1100.9653, 157.7525; 20, 1131.9018, 0.6859; 21, 1136.6667, 5.5732; 22, 1183.3154, 13.3727; 23, 1248.0601, 5.8501; 24, 1306.2454, 77.7962; 25, 1380.4356, 9.2012; 26, 1450.8076, 186.3468; 27, 1465.2826, 7.3043; 28, 1496.7858, 46.1416; 29, 1506.7736, 2.1680; 30, 1518.8423, 13.8407; 31, 1531.3978, 11.5041; 32, 1554.6429, 28.2749; 33, 1664.5727, 202.2692; 34, 2225.3356, 779.4190; 35, 2998.4082, 66.7016; 36, 3022.7308, 65.2770; 37, 3085.9633, 33.1003; 38, 3095.6576, 43.3316; 39, 3166.9826, 19.3404; 40, 3177.9051, 6.3951; 41, 3208.7961, 8.7199; 42, 3481.44, 1.8106;

\section{Calculation summary for $\mathbf{1 , 3}$ Migration of $\mathrm{NMe}_{2} 1 \mathrm{sZ}$ iZ (vacuum)}

Geometry optimisation and frequency calculation: Program version = SGI64-G98RevA.7. Method and basis set = RB3LYP/6-31G(d). Calculated Energy = -380.0218166 (hartree), -1589.2512370212 $\left(\mathrm{kJ} \mathrm{mol}^{-1}\right)$. Higher level single-point calculation: Program = SGI64-G98RevA.7. Method and basis set = RB3LYP/6-

311+G(3df,2p). Calculated Energy = -380.166129 (hartree), $-1589.854751478\left(\mathrm{~kJ} \mathrm{~mol}^{-1}\right)$. Structure definition (3D Coordinates: Atom, X coord/Å, Y coord/Å, Z coord/Å): C, -0.1220477649, -0.1964695054, 0.2845906736; N, -0.0759668494, -0.2915319058, 1.5696621933; C, 1.1572637353, -0.0576994936, -0.4386895911; N, 1.2607344742, -0.2837731326, -0.5243839781; C, 2.2515722934, 0.2669017144, 0.2381129742; O, 3.2311675839, 0.5710202041, 0.7906165653; H, 1.283740561, -0.3175952447, -1.4830007551; H, 1.0068556082, -0.2842340436, 1.9880423507; C, -2.5190871339, -0.6080021343, 0.1264575366; C, 1.4087521751, 0.5642039085, -1.702736019; H, -1.9029592736, 1.519344311, -1.4606645372; H, 2.0136213289, 0.0491503592, -2.4579827293; H, -0.436137784, 0.795335613, -2.1366196133; H, 2.9434032646, 0.2357693725, 0.6967220588; H, -3.2457043236, -0.8972559471, -0.6389983681; H, 2.3811841133, -1.4551477213, 0.8042081188. Harmonic Frequencies (Number, Frequency (cm ${ }^{-1}$ ), IR Intensity $\left(\mathrm{km} \mathrm{mol}^{-1}\right)$ ): 1, 66.8042, 0.6569; 2, 96.5372, 2.0839; 3, 120.9069, 1.3476; 4, 156.8897, 0.3810; 5, 223.4639, 1.1975; 6, 252.186, 5.6326; 7, 309.5768, 1.6187; 8, 397.8403, 6.1936; 9, 444.7569, 11.1170; 10, 494.3562, 4.1855; 11, 533.7899, 14.8831; 12, 549.7266, 40.3870; 13, 625.0419, 7.2490; 14, 717.4749, 8.6659; 15, 820.4346, 86.7347; 16, 867.0147, 1.9464; 17, 985.0081, 14.9961; 18, 1088.7831, 36.1492; 19, 1102.5621, 
78.6797; 20, 1133.9928, 6.7237; 21, 1144.7307, 0.5470; 22, 1179.4648, 14.2986; 23, 1247.9188, 99.2978; 24, 1303.3151, 27.5360; 25, 1368.8484, 59.9174; 26, 1465.9869, 194.2834; 27, 1468.508, 8.0841; 28, 1506.2354, 68.5749; 29, 1512.4365, 12.6249; 30, 1525.5222, 9.3425; 31, 1536.2105, 4.2585; 32, 1554.5379, 39.1302; 33, 1660.1814, 170.0577; 34, 2234.9809, 811.1637; 35, 2989.0572, 46.0490; 36, 3000.6297, 90.9389; 37, 3082.6248, 38.0555; 38, 3095.2998, 29.6155; 39, 3139.3063, 15.2533; 40, 3173.9499, 16.0404; 41, 3231.4481, 6.1655; 42, 3489.8556, 2.9217;

\section{Calculation summary for 1,3 Migration of $\mathrm{NMe}_{2} 3 \mathrm{sE}$ (acetonitrile)}

Geometry optimisation and frequency calculation: Program version = DEC-AXP-OSF/1-G03RevB.05. Method and basis set = RB3LYP/6-31G(d). Calculated Energy = -380.0459067 (hartree), $-1589.3519818194\left(\mathrm{~kJ} \mathrm{~mol}^{-1}\right)$. Higher level single-point calculation: Program = DEC-AXP-OSF/1-G03RevB.05. Method and basis set = RB3LYP/6-311++G(3df,2p). Calculated Energy = -380.1911037 (hartree), -1589.9591956734 (kJ mol $\left.{ }^{-1}\right)$. Structure definition (3D Coordinates: Atom, X coord/A, Y coord/Å, Z coord/A): C, 0.4508338512 , 0.823503416, 0.099650946; O, 1.1630162797, 1.8296463843, -0.0354291322; C, -1.0053093412, 1.019852331, 0.3270767671; N, 0.9676394899, -0.4423760931, 0.0288122577; C, -1.9922880225, 0.2162741922, -0.039510605; N, -2.9073439862, -0.4515137199, -0.4961194205; H, -3.3749548886, 1.1342642349, 0.1298181794; H, -1.2785419824, 2.0088197223, 0.6932750351; C, 0.266456329, 1.6630843852, 0.4057305456; C, 2.3617989654, -0.6144621673, -0.3636504425; H, 2.9715938614, 0.9395381767, 0.4899448344; H, 2.744868317, 0.3346068903, -0.7346205461; H, 2.4345771909, 1.374935195, -1.1503047848; H, 0.973990962, -2.3329786973, 0.9066060694; H, -0.1475160325, 2.1906912493, -0.4637411107; H, -0.5391668819, -1.4434617638, 1.1078222526. Harmonic Frequencies (Number, Frequency $\left(\mathrm{cm}^{-1}\right)$, IR Intensity $\left(\mathrm{km} \mathrm{mol}^{-1}\right)$ ): 1, -15.5045, 4.7663; 2, 104.8993, 3.6378; 3, 112.9525, 4.1456; 4, 156.0608, 5.8458; 5, 195.7102, 1.9144; 6, 222.0109, 6.0074; 7, 317.5583, 15.7809; 8, 406.1696, 8.8347; 9, 445.4969, 39.0796; 10, 452.2223, 59.2397; 11, 484.6528, 18.1101; 12, 543.0742, 47.1365; 13, 694.6758, 13.5312; 14, 722.3196, 30.0720; 15, 774.542, 74.6964; 16, 847.6565, 122.7367; 17, 953.0504, 590.2256; 18, 1002.2041, 38.1462; 19, 1085.717, 10.3748; 20, 1130.9942, 116.9176; 21, 1137.7365, 7.0708; 22, 1175.0036, 3.6498; 23, 1215.1968, 104.7835; 24, 1287.5575, 63.5514; 25, 1393.8637, 10.1188; 26, 1427.6587, 269.6170; 27, 1452.4078, 32.4346; 28, 1490.333, 74.5565; 29, 1515.4745, 8.3694; 30, 1517.83, 24.2060; 31, 1528.8508, 7.6652; 32, 1552.3455, 80.8836; 33, 1671.9845, 792.9090; 34, 2113.3279, 878.7289; 35, 3034.3579, 46.2204; 36, 3040.3567, 63.0985; 37, 3088.184, 49.7109; 38, 3098.4621, 15.7313; 39, 3139.5223, 39.6197; 40, 3158.821, 18.9295; 41, 3174.4828, 316.1422; 42, 3192.1696, 3.4330;

\section{Calculation summary for 1,3 Migration of $\mathrm{NMe}_{2} 3 \mathrm{sZ}$ (acetonitrile)}

Geometry optimisation and frequency calculation: Program version = DEC-AXP-OSF/1-G03RevB.05. Method and basis set = RB3LYP/6-31G(d). Calculated Energy = -380.0490743 (hartree), $-1589.3652287226\left(\mathrm{~kJ} \mathrm{~mol}^{-1}\right)$. Higher level single-point calculation: Program = DEC-AXP-OSF/1-G03RevB.05. Method and basis set =

RB3LYP/6-311++G(3df,2p). Calculated Energy = -380.1943231 (hartree), -1589.9726592042 (kJ mol ${ }^{-1}$ ). Structure definition (3D Coordinates: Atom, X coord/A, Y coord/Å, Z coord/Å): C, 0.0836647864, 0.3319572037, -0.0091824577; O, -0.2054608443, -1.5402634764, -0.0344913722; C, -0.9760051949, 0.6978702221, 0.0661807399; N, 1.3821735122, 0.1046166872, -0.0481241067; C, -2.246520836, 0.3129035237, 0.0295009819; N, -3.4108269926, -0.0052937064, -0.1270167463; H, -3.9686825168, 0.2668785095, 0.7079235197; H, -0.7839697787, 1.7567446411, 0.2066437052; C, 1.7864177632, 1.50597943, -0.0286753375; C, 2.4620907692, -0.8714422339, 0.0321224059; H, 3.1329183604, 0.770319309, -0.829896152; H, 2.0284707662, -1.8698713594, 0.0396664139; H, 3.0498295556, 0.7215010335, 0.9475525133; H, 2.7715755875, 1.5888164616, -0.4968244967; H, 1.8589537931, 1.9043699437, 0.9930796778; H, 1.0972816224, 2.1253636799, -0.6059062276. Harmonic Frequencies 
(Number, Frequency $\left(\mathrm{cm}^{-1}\right)$, IR Intensity $\left.\left(\mathrm{km} \mathrm{mol}^{-1}\right)\right): 1,-42.3825,4.9030 ; 2,81.3804,3.1133 ; 3,97.8964$, 3.7043; 4, 130.2133, 3.0379; 5, 148.4792, 5.4627; 6, 221.362, 6.0736; 7, 315.9708, 12.0785; 8, 396.7204, 6.4759; 9, 447.3839, 1.2785; 10, 451.6691, 84.2616; 11, 501.8784, 18.6645; 12, 525.6131, 49.6670; 13, 638.0595, 11.9602; 14, 754.7774, 65.4919; 15, 829.5207, 88.6768; 16, 867.8043, 20.5550; 17, 919.1763, 761.9370; 18, 992.5318, 3.1080; 19, 1086.4716, 19.2382; 20, 1132.3837, 38.5802; 21, 1142.3701, 317.1091; 22, 1171.914, 12.5275; 23, 1179.2757, 21.6262; 24, 1290.8112, 62.5716; 25, 1387.8129, 73.3782; 26, 1443.166, 132.0482; 27, 1458.8839, 264.9265; 28, 1493.2901, 92.8684; 29, 1519.4449, 13.5010; 30, 1523.8989, 7.5807; 31, 1527.8066, 24.2384; 32, 1549.1164, 125.5185; 33, 1663.5235, 415.3552; 34, 2123.3635, 1095.5313; 35, 3030.4457, 44.1035; 36, 3036.5421, 91.8155; 37, 3085.3332, 43.1471; 38, 3110.3601, 23.1894; 39, 3151, 13.9801; 40, 3175.0538, 368.9948; 41, 3193.94, 3.1424; 42, 3198.5246, 7.9872;

\section{Calculation summary for 1,3 Migration of $\mathrm{NMe}_{2}$ TS2 iE (acetonitrile)}

Geometry optimisation and frequency calculation: Program version = x86-Linux-G03RevB.01. Method and basis set = RB3LYP/6-31G(d). Calculated Energy = -380.0071967 (hartree), -1589.1900965994 (kJ mol $\left.{ }^{-1}\right)$. Higher level single-point calculation: Program = x86-Linux-G03RevB.01. Method and basis set = RB3LYP/6-

311++G(3df,2p). Calculated Energy = -380.1521028 (hartree), -1589.7960939096 $\left(\mathrm{kJ} \mathrm{mol}^{-1}\right)$. Structure definition (3D Coordinates: Atom, X coord/Å, Y coord/Å, Z coord/Å): C, 1.3818481681, 0.1068436181, 0.7903879846; C, 1.385920717, 0.1090437077, 0.5929579852; N, 1.7823459364, 0.1130057051, 1.7499919358; C, 0.0136535001, 0.0643325555, -1.134804983; N, -0.5819092559, -0.0701493872, 0.2388116167; O, -0.6500067819, 0.1137513873, -2.1604986083; H, 2.2609274227, 0.1107157607, 1.4281779735; C, -1.1322854882, -1.4028259863, 0.5180879879; C, -1.4960543387, 1.0143967102, 0.6141131887; H, 2.5851537605, 0.6767320675, 2.0610089848; H, -1.696982142, 0.9534636634, 1.6881612946; H, -1.0255884113, 1.976415501, 0.3937464029; H, -2.4454201198, 0.943650106, 0.0670755952; H, -1.3347239303, -1.4889269076, 1.5901313414; H, -2.0640486134, -1.5704234542, 0.0388685364; H, -0.4008158246, -2.162375692, 0.2294897248. Harmonic Frequencies (Number, Frequency $\left(\mathrm{cm}^{-1}\right)$, IR Intensity $\left.\left(\mathrm{km} \mathrm{mol}^{-1}\right)\right): 1,-442.6489,119.7066 ; 2,109.3153,10.0426 ; 3,126.0778,2.6728 ; 4$, 158.7245, 6.2276; 5, 198.7468, 1.6508; 6, 252.1529, 0.6936; 7, 259.6424, 7.8453; 8, 303.3963, 9.3089; 9, 391.9298, 27.6373; 10, 437.2755, 436.4099; 11, 544.1755, 75.4254; 12, 580.3562, 51.1526; 13, 647.6652, 29.1205; 14, 696.0409, 98.9056; 15, 739.4971, 4.8761; 16, 768.507, 19.7898; 17, 776.2475, 382.7152; 18, 973.5481, 149.1609; 19, 1057.5479, 11.1638; 20, 1060.8343, 8.0713; 21, 1109.7296, 1.6930; 22, 1202.0661, 2.6540; 23, 1217.7038, 21.3267; 24, 1226.2207, 100.3372; 25, 1295.6601, 20.2645; 26, 1335.1657, 34.5876; 27, 1450.5588, 0.1858; 28, 1477.8737, 2.0177; 29, 1502.1405, 3.4947; 30, 1511.5776, 7.6634; 31, 1516.3377, 13.6709; 32, 1526.1996, 19.8251; 33, 1762.677, 2058.8693; 34, 1992.6951, 819.6823; 35, 3036.0113, 32.1845; 36, 3041.0885, 62.8863; 37, 3113.4674, 15.3523; 38, 3115.974, 17.7004; 39, 3146.8682, 10.8661; 40, 3148.3554, 17.0341; 41, 3191.3738, 7.4890; 42, 3284.3997, 596.6713;

\section{Calculation summary for 1,3 Migration of $\mathrm{NMe}_{2} \mathrm{TS}^{\prime}$ iE (acetonitrile)}

Geometry optimisation and frequency calculation: Program version = x86-Linux-G03RevB.01. Method and basis set = RB3LYP/6-31G(d). Calculated Energy = -380.0246745 (hartree), -1589.263188759 $\left(\mathrm{kJ} \mathrm{mol}^{-1}\right)$. Higher level single-point calculation: Program = x86-Linux-G03RevB.01. Method and basis set = RB3LYP/6311++G(3df,2p). Calculated Energy = -380.1672971 (hartree), -1589.8596364722 (kJ mol $\left.{ }^{-1}\right)$. Structure definition (3D Coordinates: Atom, X coord/Å, Y coord/A, Z coord/Å): C, 0.8249450673, 1.3613602539, 0.0049519354; C, -0.5411759281, 0.9166875648, 0.001306898; N, -1.6940150589, 1.4606226295, 0.0053640342; C, 1.5899514283, 0.2407973045, -0.0076161092; N, -0.3813904214, -0.5505675169, 0.0022671833; O, 2.5247513046, -0.4763353693, -0.0112948504; H, 1.2274915853, 2.3713131915, 0.0075157329; C, -0.8732290909, -1.1945462648, 1.2273782691; C, -0.8862112234, -1.1976211925, - 
1.2159042875; H, -1.5990549514, 2.4883758429, 0.003762726; H, -1.9828564128, -1.1604241126, 1.2678463388; H, -0.4715386411, -0.6921711351, -2.0924043189; H, -0.5621835537, -2.2429260828, 1.2218594394; H, -1.9693110864, -1.1577553329, 1.2905738966; H, -0.5486364646, -2.239676716, 1.2327993395; H, -0.449764069, -0.6865044838, 2.0981531384. Harmonic Frequencies (Number, Frequency $\left(\mathrm{cm}^{-1}\right)$, IR Intensity $\left.\left(\mathrm{km} \mathrm{mol}^{-1}\right)\right): 1,-132.0779,77.1031 ; 2$, 74.5862, 6.4219; 3, 136.5339, 3.3521; 4, 214.9238, 11.5246; 5, 217.9189, 0.1909; 6, 255.9529, 0.4627; 7, 287.6956, 19.3397; 8, 357.8427, 153.7948; 9, 380.0767, 10.0307; 10, 514.2361, 8.3235; 11, 587.7798, 13.0800; 12, 652.9967, 4.2275; 13, 672.1298, 44.1922; 14, 739.7031, 0.0344; 15, 747.7106, 30.1750; 16, 848.5904, 173.3329; 17, 985.0497, 96.7020; 18, 1061.8692, 9.8496; 19, 1062.5332, 62.9180; 20, 1114.4408, 3.7452; 21, 1114.7664, 35.0309; 22, 1203.9775, 1.7083; 23, 1225.3274, 11.2457; 24, 1285.1082, 60.1040; 25, 1333.3381, 68.5604; 26, 1357.6002, 179.0360; 27, $1453.5143,0.0275 ; 28,1482.1875,4.0117 ; 29,1501.6592$, 4.2797; 30, 1514.9092, 18.8011; 31, 1515.5017, 6.1577; 32, 1529.951, 19.3151; 33, 1716.874, 1017.7296; 34, 2079.2421, 1401.3557; 35, 3033.444, 29.5456; 36, 3037.4981, 71.1167; 37, 3111.0183, 4.7139; 38, 3111.4541, 38.8371; 39, 3144.0225, 12.3466; 40, 3145.7853, 24.3604; 41, 3175.2995, 14.6982; 42, 3278.0724, 51.4293;

\section{Calculation summary for 1,3 Migration of $\mathrm{NMe}_{2}$ Intermediate iE (acetonitrile)}

Geometry optimisation and frequency calculation: Program version $=$ x86-Linux-G03RevB.01. Method and basis set $=$ RB3LYP/6-31G(d). Calculated Energy = -380.0281999 (hartree), $-1589.2779319818\left(\mathrm{~kJ} \mathrm{~mol}^{-1}\right)$. Higher level single-point calculation: Program = x86-Linux-G03RevB.01. Method and basis set $=$ RB3LYP/6-

$311++\mathrm{G}(3 \mathrm{df}, 2 \mathrm{p})$. Calculated Energy = -380.1714796 (hartree), $-1589.8771276872\left(\mathrm{~kJ} \mathrm{~mol}^{-1}\right)$. Structure definition (3D Coordinates: Atom, X coord/Å, Y coord/Å, Z coord/Å): C, 0.0612272796, -1.6156765238, 0.0236029794; C, -0.9934269676, -0.6544585021, -0.0127481789; N, -2.2493824856, -0.4653007477, 0.0137800395; C, 1.1002724144, -0.6932523544, -0.0066097727; N, -0.023979771, 0.5210742151, 0.0075207906; O, 2.293238965, -0.4945678093, 0.0001861827; H, 0.0639720885, -2.6988823352, 0.0395488554; C, -0.0377973211, 1.3645228239, -1.2099467643; C, -0.0463030078, 1.328695763, 1.2489170979; H, -2.7414035512, -1.371961573, -0.0282561232; H, -0.9491332454, 1.9445552594, 1.2689973071; H, -0.0390898101, 0.6536778874, 2.1071317585; H, 0.8430035739, 1.964027723, 1.269439418; H, -0.9401677714, 1.9813538032, -1.2179781798; H, 0.8519285623, 1.9995716795, 1.2061136424; H, -0.0253201546, 0.714798518, -2.0874028184. Harmonic Frequencies (Number, Frequency $\left(\mathrm{cm}^{-1}\right)$, IR Intensity $\left(\mathrm{km} \mathrm{mol}^{-1}\right)$ ): 1, 138.0286, 10.5709; 2, 172.3181, 0.5431; 3, 176.0079, 0.1382; 4, 201.8957, 0.2248; 5, 244.4885, 3.8294; 6, 264.8428, 0.0171; 7, 286.0044, 7.2039; 8, 407.1479, 9.7626; 9, 546.4349, 140.4438; 10, 601.4042, 16.0374; 11, 655.3004, 1.7676; 12, 675.9441, 1.8845; 13, 716.1942, 54.2980; 14, 725.615, 22.7869; 15, 730.1265, 3.1995; 16, 800.6616, 180.2019; 17, 965.8571, 114.0432; 18, 1010.8883, 43.1050; 19, 1041.1878, 6.6055; 20, 1113.7085, 0.4936; 21, 1152.3653, 26.9596; 22, 1217.585, 4.0974; 23, 1227.7333, 2.2321; 24, 1284.6664, 76.8997; 25, 1298.1057, 76.8995; 26, 1306.3368, 100.8336; 27, 1446.5087, 1.7605; 28, 1477.9403, 8.3338; 29, 1501.7559, 2.3315; 30, 1505.24, 9.0855; 31, 1517.8708, 23.8588; 32, 1524.4472, 16.3023; 33, 1723.5764, 1909.8946; 34, 1904.6407, 558.9915; 35, 3073.374, 9.7861; 36, 3077.2951, 13.5525; 37, 3158.9829, 0.0012; 38, 3161.615, 9.4092; 39, 3170.3607, 3.9990; 40, 3171.6863, 6.3031; 41, 3227.6714, 9.3390; 42, 3285.7173, 91.0454;

\section{Calculation summary for 1,3 Migration of $\mathrm{NMe}_{2}$ TS2 iZ (acetonitrile)}

Geometry optimisation and frequency calculation: Program version = x86-Linux-G03RevB.01. Method and basis set $=$ RB3LYP/6-31G(d). Calculated Energy = -380.0123739 (hartree), $-1589.2117476498\left(\mathrm{~kJ} \mathrm{~mol}^{-1}\right)$. Higher level single-point calculation: Program = x86-Linux-G03RevB.01. Method and basis set $=$ RB3LYP/6$311++G(3 d f, 2 p)$. Calculated Energy = -380.1567709 (hartree), -1589.8156159038 $\left(\mathrm{kJ} \mathrm{mol}^{-1}\right)$. Structure definition (3D Coordinates: Atom, X coord/Å, Y coord/Å, Z coord/Å): C, 1.105538605, 0.0187073527, - 
1.1600908271; C, 1.1023699181, 0.0013888963, 0.2491944479; O, 1.9359382699, -0.0173080016, 1.1435721287; C, -0.2360978387, -0.0318205389, -1.4971339904; N, -1.1583431272, -0.1798324272, 2.289043992; N, -0.3842746519, 0.0163471281, 0.5037897802; H, 1.9377391108, 0.0922285582, 1.8532261824; C, -0.8573732855, 1.2787208866, 1.0898768174; C, -0.8749315793, -1.1641869336, 1.2278089134; H, -2.1151821201, 0.170819853, -2.1723330366; H, -1.9516376557, 1.2926588529, 1.0820622162; H, -0.5044282404, 1.3882936792, 2.1240599591; H, -0.4826375437, 2.115127146, 0.493596044; H, -1.9690795808, -1.1630806335, 1.215731155; H, -0.5129984144, -2.0683046451, 0.7309217075; H, -0.5279921784, -1.1617396829, 2.2694584226. Harmonic Frequencies (Number, Frequency

$\left(\mathrm{cm}^{-1}\right)$, IR Intensity $\left.\left(\mathrm{km} \mathrm{mol}^{-1}\right)\right): 1,-359.1135,320.4182 ; 2,109.0064,5.0827 ; 3,132.8264,2.5891 ; 4$, 180.3016, 4.5770; 5, 206.6767, 3.7348; 6, 251.9407, 5.8293; 7, 266.2598, 29.5517; 8, 301.9604, 33.5516; 9, 386.2566, 11.2948; 10, 445.2624, 272.5771; 11, 542.9553, 65.1491; 12, 594.9264, 29.7441; 13, 651.7216, 1.1236; 14, 696.7071, 152.8906; 15, 734.3776, 66.0026; 16, 766.2482, 23.6822; 17, 803.379, 727.4207; 18, 967.6999, 127.0044; 19, 1057.1232, 10.1801; 20, 1061.4933, 26.2732; 21, 1110.3455, 1.5690; 22, 1204.3266, 5.9225; 23, 1215.0682, 20.0085; 24, 1228.0146, 128.0503; 25, 1290.3828, 16.3377; 26, 1338.3066, 29.8353; 27, 1450.3671, 0.1326; 28, 1478.5096, 0.1663; 29, 1502.728, 3.4481; 30, 1512.5164, 7.4713; 31, 1516.8238, 16.6031; 32, 1526.8777, 19.2733; 33, 1764.5736, 1980.9920; 34, 1997.5139, 665.7856; 35, 3037.3754, 28.8017; 36, 3042.0317, 60.0292; 37, 3114.0695, 14.4502; 38, 3117.1044, 16.8339; 39, 3146.2457, 11.4105; 40, 3148.4426, 16.0519; 41, 3197.2936, 9.9208; 42, 3334.0607, 546.9021;

\section{Calculation summary for 1,3 Migration of $\mathrm{NMe}_{2}$ TS2' iZ (acetonitrile)}

Geometry optimisation and frequency calculation: Program version = x86-Linux-G03RevB.01. Method and basis set = RB3LYP/6-31G(d). Calculated Energy = -380.0221687 (hartree), -1589.2527095034 (kJ mol $\left.{ }^{-1}\right)$. Higher level single-point calculation: Program = x86-Linux-G03RevB.01. Method and basis set $=$ RB3LYP/6311++G(3df,2p). Calculated Energy = -380.1655953 (hartree), -1589.8525195446 $\left(\mathrm{kJ} \mathrm{mol}^{-1}\right)$. Structure definition (3D Coordinates: Atom, X coord/Å, Y coord/A, Z coord/Å): C, 0.7807864611, 1.4010666526, 0.0004636953; C, 1.547657952, 0.2778345378, -0.0020682159; O, 2.505145128, -0.4125536919, 0.0037629739; C, -0.5726574064, 0.9334129166, 0.0011908164; N, -1.6790479185, 1.5681338262, 0.0030531593; N, -0.3514291945, -0.5389068529, 0.0002182625; H, 1.1677363613, 2.4161378001, 0.0004956211; C, -0.7985671804, -1.2155592771, 1.2258517527; C, -0.8021925124, -1.2146205604, 1.2245775182; H, -2.4908277861, 0.9360158332, 0.0037763321; H, -1.8952609798, -1.2630129603, 1.2887017482; H, -0.4022028505, -2.2353795907, 1.2309088834; H, -0.4131640411, -0.6779103016, 2.0961121903; H, -1.8990704297, -1.2619767718, -1.2842700322; H, -0.4192995288, -0.6763820772, 2.0955773853; H, -0.4058958619, -2.2344568275, -1.2315511157. Harmonic Frequencies (Number, Frequency $\left(\mathrm{cm}^{-1}\right)$, IR Intensity $\left.\left(\mathrm{km} \mathrm{mol}^{-1}\right)\right): 1,-135.2412,77.2653 ; 2,103.4671,1.6610 ; 3,141.4548,1.2396 ; 4,227.161$, 2.6819; 5, 227.1999, 2.2213; 6, 252.8182, 3.3936; 7, 294.2554, 3.5893; 8, 354.1287, 144.4555; 9, 388.5389, 53.6838; 10, 520.5631, 16.9487; 11, 595.3709, 15.0671; 12, 636.7765, 11.7815; 13, 678.8303, 120.8917; 14, 735.7736, 3.2897; 15, 740.2481, 6.4092; 16, 827.0312, 51.7040; 17, 976.6274, 101.7028; 18, 1059.9625, 15.1680; 19, 1060.1338, 30.9683; 20, 1113.7533, 343.5373; 21, 1117.8155, 2.3444; 22, 1202.8781, 3.6184; 23, 1222.991, 22.8067; 24, 1293.394, 26.1490; 25, 1307.2821, 94.7409; 26, 1362.5782, 66.6994; 27, 1457.2028, 0.0672; 28, 1483.8813, 1.4605; 29, 1502.6581, 2.1458; 30, 1515.8297, 10.5041; 31, 1517.2721, 18.9742; 32, 1529.2979, 19.6639; 33, 1724.9144, 990.7535; 34, 2065.6458, 1351.2213; 35, 3026.4719, 22.5434; 36, 3029.505, 71.4147; 37, 3110.6403, 4.5530; 38, 3111.242, 27.2877; 39, 3149.4374, 8.9681; 40, 3151.0388, 16.1605; 41, 3186.6641, 14.7873; 42, 3337.1639, 45.9678;

\section{Calculation summary for 1,3 Migration of $\mathrm{NMe}_{2}$ Intermediate iZ (acetonitrile)}

Geometry optimisation and frequency calculation: Program version = x86-Linux-G03RevB.01. Method and 
basis set = RB3LYP/6-31G(d). Calculated Energy = -380.0248501 (hartree), -1589.2639231182 (kJ mol $\left.{ }^{-1}\right)$. Higher level single-point calculation: Program = x86-Linux-G03RevB.01. Method and basis set = RB3LYP/6-

311++G(3df,2p). Calculated Energy = -380.1690614 (hartree), -1589.8670147748 $\left(\mathrm{kJ} \mathrm{mol}^{-1}\right)$. Structure definition (3D Coordinates: Atom, X coord/Å, Y coord/Å, Z coord/Å): C, 1.1355401811, -0.043169226, 1.158334759; C, 1.2803765081, -0.0030105348, 0.2213980204; O, 2.0248954574, 0.0199815527, 1.1736728434; C, -0.2874903999, -0.0336850487, -1.176729172; N, -1.2320057661, -0.0531378449, 2.0246536792; N, -0.3708557037, 0.0141868731, 0.3676425358; H, 1.8701026965, -0.0734841311, 1.9538151094; C, -0.9166284066, 1.2673074024, 0.9373646582; C, -0.9354991624, -1.1933833666, 1.0124760096; H, -2.1699716767, -0.0333541659, -1.6035191338; H, -2.0046524486, 1.2886589631, 0.8218906722; H, -0.6583629183, 1.3099540028, 1.9989149453; H, -0.4703513534, 2.1155110879, 0.4141922187; H, -2.0238883048, -1.2043827369, 0.8991552816; H, -0.5035085735, -2.0786628574, 0.5413345012; H, -0.6762931138, -1.1757911391, 2.0744933383. Harmonic Frequencies (Number, Frequency $\left(\mathrm{cm}^{-1}\right)$, IR Intensity $\left.\left(\mathrm{km} \mathrm{mol}^{-1}\right)\right): 1,141.7639,4.6992 ; 2,176.2013,0.5637 ; 3,177.1148,0.0266 ; 4,203.2916$, 0.0078; 5, 245.8247, 0.7254; 6, 260.7093, 6.9257; 7, 286.4596, 5.2254; 8, 409.5568, 0.0348; 9, 508.667, 146.5095; 10, 609.8458, 0.6667; 11, 635.3615, 23.6179; 12, 674.8458, 3.0499; 13, 707.3324, 168.7688; 14, 710.8484, 19.3536; 15, 727.7709, 25.7195; 16, 760.3048, 11.5897; 17, 957.3817, 141.6827; 18, 1017.2198, 41.5270; 19, 1040.2924, 12.9533; 20, 1115.125, 0.1434; 21, 1141.3221, 367.7256; 22, 1214.3092, 10.1208; 23, 1224.225, 0.5373; 24, 1267.368, 108.4747; 25, 1299.4163, 32.4304; 26, 1305.0809, 7.6640; 27, 1449.7646, 2.2430; 28, 1479.3778, 3.2196; 29, 1501.4979, 0.0004; 30, 1506.7293, 12.2655; 31, 1520.7673, 24.3621; 32, 1522.2551, 18.8107; 33, 1740.1497, 1840.4703; 34, 1912.0057, 522.5268; 35, 3066.9977, 8.1277; 36, 3070.6746, 15.0421; 37, 3148.6173, 0.2117; 38, 3150.9161, 8.3665; 39, 3168.9839, 3.2483; 40, 3170.2055, 4.5566; 41, 3228.9693, 9.8083; 42, 3330.5303, 90.3592;

\section{Calculation summary for 1,3 Migration of $\mathrm{NMe}_{2} 1 \mathrm{sE}$ iE (acetonitrile)}

Geometry optimisation and frequency calculation: Program version = x86-Linux-G03RevB.01. Method and basis set = RB3LYP/6-31G(d). Calculated Energy = -380.0331549 (hartree), -1589.2986537918 $\left(\mathrm{kJ} \mathrm{mol}^{-1}\right)$. Higher level single-point calculation: Program = x86-Linux-G03RevB.01. Method and basis set = RB3LYP/6311++G(3df,2p). Calculated Energy = -380.178128 (hartree), -1589.904931296 $\left(\mathrm{kJ} \mathrm{mol}^{-1}\right)$. Structure definition (3D Coordinates: Atom, X coord/Ä, Y coord/Å, Z coord/Å): C, 0.1445410881, 0.0909753579, 0.8879980982; N, 0.0197485525, 0.0060577022, 2.1684907672; C, 1.4640244274, 0.1661293971, 0.2083196631; N, 0.9793525008, 0.0317311044, 0.0652501422; C, 1.6974451945, -0.3703666491, -0.9767799379; O, 1.8989239125, -0.8366142145, -2.0312344024; H, 2.3266125067, 0.553407378, 0.7502763965; C, 1.1355447704, 0.9577390705, -1.0585861238; C, -2.2475615571, -0.2740192425, 0.7181377661; H, 2.6522411998, 0.5882863433, 1.2698536465; H, -2.1095508314, -1.0936380112, 1.4245600616; H, 2.9702687453, -0.5684010471, -0.0498827303; H, -1.6676390051, 1.8709497097, -0.7480162906; H, 1.7157220694, 0.4782280108, -1.8545409241; H, -0.1677533959, 1.2539635495, -1.4620739059; H, 0.9449727826, -0.0771514673, 2.6089758061. Harmonic Frequencies (Number, Frequency ( $\mathrm{cm}^{-1}$ ), IR Intensity $\left.\left(\mathrm{km} \mathrm{mol}^{-1}\right)\right):$ 1, 72.8069, 1.9072; 2, 88.2436, 7.3932; 3, 114.2837, 5.5650; 4, 165.9131, 4.7399; 5, 200.012, 2.3450; 6, 267.4038, 26.0017; 7, 319.0728, 8.2074; 8, 400.4752, 1.0286; 9, 448.8312, 3.5512; 10, 486.3969, 3.7320; 11, 523.9102, 3.9598; 12, 546.8376, 57.9827; 13, 694.199, 2.6164; 14, 712.2889, 76.9711; 15, 762.0998, 7.0883; 16, 818.4893, 114.6817; 17, 996.6339, 16.0669; 18, 1080.7883, 30.5955; 19, 1102.2505, 174.7380; 20, 1134.1914, 13.4084; 21, 1140.4071, 76.2948; 22, 1180.4982, 17.8727; 23, 1243.6102, 9.5534; 24, 1304.7457, 183.4617; 25, 1380.3136, 11.3063; 26, 1422.4596, 350.5709; 27, 1463.449, 3.0239; 28, 1492.1898, 49.4241; 29, 1500.7913, 5.0522; 30, 1516.455, 38.0888; 31, 1525.1027, 8.2665; 32, 1546.157, 58.2254; 33, 1645.4426, 480.7449; 34, 2182.9316, 1342.3002; 35, 3005.5801, 67.7500; 36, 3015.53, 92.4642; 37, 3090.6139, 40.6253; 38, 3097.2677, 39.5757; 39, 3132.7225, 58.9207; 40, 3165.3516, 15.1420; 41, 3176.6834, 15.8072; 42, 3330.4776, 38.3827; 
Calculation summary for 1,3 Migration of $\mathrm{NMe}_{2} 1 \mathrm{sE}$ iZ (acetonitrile)

Geometry optimisation and frequency calculation: Program version = x86-Linux-G03RevB.01. Method and basis set = RB3LYP/6-31G(d). Calculated Energy = -380.0298877 (hartree), -1589.2849903614 (kJ mol $\left.{ }^{-1}\right)$. Higher level single-point calculation: Program = x86-Linux-G03RevB.01. Method and basis set $=$ RB3LYP/6311++G(3df,2p). Calculated Energy = -380.1751486 (hartree), -1589.8924714452 (kJ mol $\left.{ }^{-1}\right)$. Structure definition (3D Coordinates: Atom, X coord/Å, Y coord/A, Z coord/A): C, 0.37355172, 0.8363307856, 0.1104103218; N, 1.0607995689, 1.9269023126, 0.0503119668; C, -1.0811684976, 0.9786436385, 0.3414732613; N, 0.8428260741, -0.4629129502, -0.1112194259; C, -1.9882697799, 0.129590299, 0.1102260375; O, -2.8004545239, -0.6241759235, -0.4862055431; H, -1.4528376808, 1.9101832691, 0.7660506114; H, 2.0629409909, 1.7382947812, -0.0417973717; C, 2.1951891828, -0.6071426197, 0.6339991773; C, 0.4578538538, -1.5646433322, 0.7767558633; H, 1.2165956767, -1.720493866, 1.5590470225; H, 0.3602585002, -2.4928683342, 0.2028366194; H, -0.491148664, -1.3542863868, 1.2690907931; H, 2.9713146564, -0.4335874052, 0.1294666381; H, 2.314678708, -1.6268806, 1.0121908377; H, 2.3535156284, 0.0884477659, -1.4629923068. Harmonic Frequencies (Number, Frequency $\left(\mathrm{cm}^{-1}\right)$, IR Intensity $\left.\left(\mathrm{km} \mathrm{mol}^{-1}\right)\right): 1,63.685,2.2402 ; 2,94.7031,4.5690 ; 3,107.9868,6.9353 ; 4,170.8401$, 1.4794; 5, 228.8751, 2.1798; 6, 259.363, 8.0474; 7, 325.7496, 7.3505; 8, 399.7985, 6.1377; 9, 445.9375, 12.8723; 10, 480.5555, 32.8314; 11, 523.7242, 3.9308; 12, 546.6217, 72.4435; 13, 687.115, 16.3918; 14, 705.0182, 37.5544; 15, 755.9422, 8.8150; 16, 822.2674, 96.2839; 17, 978.7914, 92.7707; 18, 1081.6593, 74.4812; 19, 1096.0905, 105.7761; 20, 1132.7128, 4.0075; 21, 1133.9403, 30.4267; 22, 1174.7962, 26.8238; 23, 1241.39, 93.3603; 24, 1302.8747, 53.1214; 25, 1372.725, 33.9197; 26, 1420.3251, 284.8760; 27 , 1465.8564, 4.1559; 28, 1496.685, 19.4562; 29, 1509.2792, 15.2574; 30, 1519.9451, 19.6226; 31, 1528.344, 3.2887; 32, 1545.7887, 42.6759; 33, 1639.4373, 526.5516; 34, 2187.2061, 1331.0796; 35, 2997.1945, 57.8973; 36, 3013.0305, 82.3885; 37, 3089.3864, 41.7884; 38, 3096.8241, 23.3734; 39, 3139.7256, 19.8871; 40, 3141.9673, 60.6954; 41, 3176.8185, 13.2495; 42, 3437.9107, 9.1290;

\section{Calculation summary for $\mathbf{1 , 3}$ Migration of $\mathrm{NMe}_{2} 1 \mathrm{sZ}$ iE (acetonitrile)}

Geometry optimisation and frequency calculation: Program version = x86-Linux-G03RevB.01. Method and basis set = RB3LYP/6-31G(d). Calculated Energy = -380.0330259 (hartree), -1589.2981143138 (kJ mol ${ }^{-1}$ ). Higher level single-point calculation: Program = x86-Linux-G03RevB.01. Method and basis set = RB3LYP/6311++G(3df,2p). Calculated Energy = -380.1781028 (hartree), -1589.9048259096 (kJ mol $\left.{ }^{-1}\right)$. Structure definition (3D Coordinates: Atom, X coord/Å, Y coord/Å, Z coord/Å): C, -0.124118445, -0.3260361274, 0.3252975981; N, -0.2366843923, -0.523146787, 1.5992596614; C, 1.1612003628, -0.1611947465, 0.4081266824; N, -1.2541865507, -0.3207205513, -0.4757300818; C, 2.2079665424, 0.4251070893, 0.1441334522; O, 3.1355380462, 0.9497563149, 0.6267181227; H, 1.3028940815, -0.5301645047, 1.4239393373; H, 0.6887710554, -0.6707648209, 2.0194200884; C, -2.5321431128, -0.5928522492, 0.1692794137; C, -1.3581453063, 0.5305076039, -1.6566340894; H, -1.7837689483, 1.5158943378, 1.4084915579; H, -2.0119552706, 0.0528436279, -2.3944956616; H, -0.3807487708, 0.6896612705, 2.1115466635; H, -2.9184015756, 0.2822418808, 0.7140072742; H, -3.2585631553, -0.8745822062, 0.5998403447; H, -2.4149954316, -1.4092981563, 0.8827360107. Harmonic Frequencies (Number, Frequency $\left(\mathrm{cm}^{-1}\right)$, IR Intensity $\left.\left(\mathrm{km} \mathrm{mol}^{-1}\right)\right): 1,63.4936,0.3773 ; 2,94.109,3.7663 ; 3,121.447,3.9750 ; 4,153.4149$, 5.7360; 5, 185.3687, 2.2668; 6, 246.7799, 33.2104; 7, 317.3034, 4.7637; 8, 400.3481, 7.1536; 9, 457.9544, 10.1894; 10, 498.3777, 8.4135; 11, 533.1823, 8.9356; 12, 547.321, 30.0333; 13, 636.0874, 41.5112; 14 , 724.5437, 22.4476; 15, 803.6766, 88.4570; 16, 850.0654, 22.5468; 17, 975.2146, 5.0197; 18, 1083.4062, 65.7129; 19, 1098.5506, 312.7602; 20, 1129.5618, 5.0404; 21, 1134.4527, 9.6199; 22, 1179.616, 18.7090; 23, 1252.906, 14.9445; 24, 1307.3785, 142.6828; 25, 1383.2516, 17.6890; 26, 1449.3885, 290.3387; 27, 
1463.7189, 31.1351; 28, 1493.5415, 77.8291; 29, 1502.4929, 3.6495; 30, 1512.5087, 30.9516; 31, 1526.2758, 12.5092; 32, 1546.3319, 62.9732; 33, 1634.759, 343.5312; 34, 2189.0347, 1349.1007; 35, 3004.1784, 72.3071; 36, 3015.9747, 93.7169; 37, 3090.2386, 39.8258; 38, 3097.7916, 44.1986; 39, 3131.4005, 50.1639; 40, 3168.0209, 12.6865; 41, 3173.896, 20.1647; 42, 3346.2018, 19.8217;

\section{Calculation summary for 1,3 Migration of $\mathrm{NMe}_{2} 1 \mathrm{sZ}$ iZ (acetonitrile)}

Geometry optimisation and frequency calculation: Program version = x86-Linux-G03RevB.01. Method and basis set = RB3LYP/6-31G(d). Calculated Energy = -380.0326181 (hartree), -1589.2964088942 $\left(\mathrm{kJ} \mathrm{mol}^{-1}\right)$. Higher level single-point calculation: Program = x86-Linux-G03RevB.01. Method and basis set = RB3LYP/6-

311++G(3df,2p). Calculated Energy = -380.1779316 (hartree), -1589.9041099512 (kJ mol $\left.{ }^{-1}\right)$. Structure definition (3D Coordinates: Atom, X coord/Å, Y coord/Å, Z coord/Å): C, -0.119251994, -0.2102293314, 0.2796753044; N, -0.0595361016, -0.3603585654, 1.5664448497; C, 1.1572775453, -0.0473637701, 0.4471777027; N, -1.2608406093, -0.2563654667, -0.5073237567; C, 2.2446483837, 0.2831076874, 0.232227218; O, 3.2150679334, 0.5896881012, 0.8056627871; H, 1.2921879169, -0.2618347696, 1.5048702372; H, -0.9877765359, -0.3555534052, 1.9957024831; C, -2.526120717, -0.5776853055, 0.137744554; C, -1.4004680965, 0.5463663651, -1.7208711691; H, -1.9087617624, 1.5002266001, 1.5121055907; H, -1.99231299, -0.0029261295, -2.4614067413; H, -0.426618092, 0.7724728517, 2.1536475587; H, -2.9363464281, 0.2640842865, 0.7181602017; H, -3.2529277893, -0.8455709079, 0.634444688; H, -2.4018615392, -1.4365089832, 0.8038729546. Harmonic Frequencies (Number, Frequency $\left(\mathrm{cm}^{-1}\right)$, IR Intensity $\left.\left(\mathrm{km} \mathrm{mol}^{-1}\right)\right): 1,61.9411,1.1962 ; 2,93.8025,4.6544 ; 3,107.1219,1.9987 ; 4,151.6326$, 0.9822; 5, 211.9846, 1.2802; 6, 237.6473, 11.3999; 7, 313.7438, 2.2774; 8, 400.3537, 11.0556; 9, 448.5207, 19.8150; 10, 495.7128, 7.3633; 11, 538.2259, 5.3401; 12, 548.1986, 81.9885; 13, 621.5769, 12.1188; 14, 713.1649, 8.2899; 15, 791.6339, 150.9896; 16, 861.0584, 8.2162; 17, 975.3022, 23.5038; 18, 1086.5073, 53.3797; 19, 1106.8021, 136.2225; 20, 1131.8137, 10.6919; 21, 1141.6833, 0.9783; 22, 1174.7422, 24.0033; 23, 1243.0547, 181.3055; 24, 1301.4376, 63.5455; 25, 1374.3561, 94.2213; 26, 1460.1575, 332.6517; 27 , 1468.8195, 10.1882; 28, 1504.2554, 75.9402; 29, 1505.9545, 23.1017; 30, 1519.3698, 13.2060; 31, 1529.2247, 3.6298; 32, 1546.9005, 77.2999; 33, 1623.9274, 323.6887; 34, 2191.9724, 1393.0075; 35, 3004.4349, 50.0893; 36, 3014.2512, 92.5895; 37, 3090.3775, 43.3633; 38, 3097.8579, 23.6956; 39, 3138.8533, 16.2781; 40, 3161.1589, 41.2177; 41, 3180.7351, 19.5481; 42, 3452.5136, 5.6160;

\section{1,3 Migration of $\mathrm{Ph}$}

\section{Calculation summary for 1,3 Migration of Ph 3 sE (vacuum)}

Geometry optimisation and frequency calculation: Program version = x86-Linux-G03RevB.01. Method and basis set = RB3LYP/6-31G(d). Calculated Energy = -477.0994122 (hartree), -1995.2297418204 $\left(\mathrm{kJ} \mathrm{mol}^{-1}\right)$. Higher level single-point calculation: Program = x86-Linux-G03RevB.01. Method and basis set = RB3LYP/6311+G(3df,2p). Calculated Energy = -477.2676597 (hartree), -1995.9333528654 (kJ mol $\left.{ }^{-1}\right)$. Structure definition (3D Coordinates: Atom, X coord/Å, Y coord/Å, Z coord/Å): C, 2.0627246065, 0.2122946197, 1.0391651689; C, 2.587181176, -0.3287022076, 0.0502335705; N, 3.0981721966, -0.7152612202, 1.0905692444; C, 0.6214883341, 0.3177579195, -1.3720030266; C, -0.424300847, 0.1631115425, 0.307934862; O, 0.2993553178, 0.5160776684, -2.537999347; H, 2.7415339784, 0.490190735, -1.840797199; H, 3.2818782129, -1.7153463997, 1.1949145967; C, -1.6935269064, -0.2818568269, -0.7101220455; C, 2.7154830481, -0.4342135287, 0.2219754676; C, -2.4883342537, -0.1216509022, 1.5657007556; C, 1.2369608947, 0.345555864, 1.9699664676; C, -0.206583148, 0.485658445, 1.0392885871; H, 1.8545872025, -0.5019100392, -1.7604441837; H, -3.6911354153, -0.7909329134, -0.096901989; H, 3.2875465672, -0.2345992854, 2.2935397407; H, -1.0627037324, 0.6088135542, 3.0096617701; H, 
0.7532826951, 0.87426199, 1.3623988574. Harmonic Frequencies (Number, Frequency ( $\mathrm{cm}^{-1}$ ), IR Intensity $\left(\mathrm{km} \mathrm{mol}^{-1}\right)$ ): 1, 46.901, 0.2792; 2, 97.1089, 4.2107; 3, 115.3326, 4.1139; 4, 171.3198, 2.5015; 5, 213.1373, 0.9840; 6, 346.9939, 2.8733; 7, 399.4293, 11.7141; 8, 419.3884, 0.4732; 9, 456.046, 38.2718; 10, 481.5463, 4.7662; 11, 517.5717, 30.1794; 12, 558.0383, 5.0234; 13, 631.6961, 2.8344; 14, 659.8545, 26.9993; 15, 707.2281, 11.0804; 16, 735.0179, 67.3760; 17, 748.6657, 11.7484; 18, 816.7929, 0.4775; 19, 865.4206, 1.9951; 20, 868.3107, 70.6897; 21, 946.9318, 1.1987; 22, 974.7856, 395.0551; 23, 980.205, 3.7853; 24, 1004.5236, 0.3679; 25, 1018.3614, 1.3977; 26, 1048.9927, 21.7364; 27, 1078.0926, 37.2221; 28, 1115.8922, 2.1671; 29, 1150.6909, 5.0235; 30, 1194.5037, 0.2029; 31, 1212.9613, 10.8554; 32, 1297.053, 226.5220; 33, 1346.6745, 3.7640; 34, 1367.6804, 3.0605; 35, 1396.273, 44.1594; 36, 1493.3336, 14.2928; 37, 1540.232, 1.0321; 38, 1637.4308, 9.7609; 39, 1658.8964, 15.7733; 40, 1733.2047, 284.2672; 41, 2127.227, 507.0051; 42, 3185.5293, 0.1949; 43, 3196.7545, 14.4363; 44, 3202.2583, 3.6454; 45, 3207.6378, 25.8779; 46, 3223.4296, 8.0960; 47, 3225.4174, 4.1187; 48, 3473.4393, 24.9406;

\section{Calculation summary for 1,3 Migration of $\mathrm{Ph} 3 \mathrm{sZ}$ (vacuum)}

Geometry optimisation and frequency calculation: Program version = x86-Linux-G03RevB.01. Method and basis set $=$ RB3LYP/6-31G(d). Calculated Energy = -477.099984 (hartree), $-1995.232133088\left(\mathrm{~kJ} \mathrm{~mol}^{-1}\right)$. Higher level single-point calculation: Program = x86-Linux-G03RevB.01. Method and basis set $=$ RB3LYP/6311+G(3df,2p). Calculated Energy = -477.2682146 (hartree), $-1995.9356734572\left(\mathrm{~kJ} \mathrm{~mol}^{-1}\right)$. Structure definition (3D Coordinates: Atom, X coord/Å, Y coord/Å, Z coord/Å): C, 0.8679815985, 0.1576762757, 1.7816716103; C, 0.7687918299, 0.0660678652, 3.103145013; H, 1.8275657831, 0.4415297647, 1.3664888791; N, 0.6779756054, -0.0177724604, 4.3123777828; C, -0.3258647301, -0.0665920105, 0.9394419924; O, -1.4234505341, -0.2992068544, 1.4386255484; C, -0.1651097845, -0.0009390663, 0.5529639964; H, 0.8355342432, -0.9222545697, 4.7586302366; C, -1.3381045843, -0.0050203571, 1.3246479324; C, -1.2726424305, 0.0510899303, -2.7133291947; C, -0.0314111448, 0.1025168562, 3.354257165; C, 1.1415889999, 0.096572147, -2.5979982597; C, 1.0756289472, 0.0478220957, 1.2055310235; H, -2.2898311421, -0.0523314759, -0.8061097833; H, -2.1881491985, 0.0536282837, 3.2987588532; H, 0.0210763655, 0.144780621, -4.4389645864; H, 2.1092162878, 0.1282932801, 3.0913090068; H, 2.0012104883, 0.0292537374, -0.6388120163. Harmonic Frequencies (Number, Frequency $\left(\mathrm{cm}^{-1}\right)$, IR Intensity $\left.\left(\mathrm{km} \mathrm{mol}^{-1}\right)\right): 1$, 28.988, 6.1693; 2, 54.4894, 2.4877; 3, 110.4155, 0.4416; 4, 170.1178, 1.4151; 5, 227.5028, 3.9505; 6, 328.3855, 2.6560; 7, 412.2631, 2.1011; 8, 433.7609, 6.2280; 9, 456.0036, 50.0965; 10, 464.1669, 4.1056; 11, 477.5891, 5.6177; 12, 532.7624, 36.0505; 13, 632.1278, 0.6927; 14, 690.0955, 26.5154; 15, 698.956, 2.5921; 16, 727.9518, 88.8133; 17, 814.6852, 13.9563; 18, 838.054, 45.2703; 19, 861.7618, 2.4393; 20, 871.6275, 23.7163; 21, 938.7435, 555.2948; 22, 940.981, 2.1092; 23, 981.0348, 0.3070; 24, 1009.2769, 3.1233; 25, 1015.7394, 60.7503; 26, 1019.35, 12.0364; 27, 1059.7242, 0.9696; 28, 1113.3552, 2.9730; 29, 1162.1634, 14.8098; 30, 1193.6983, 3.8502; 31, 1218.4667, 24.0284; 32, 1237.3492, 149.8739; 33, 1350.3333, 2.0579; 34, 1368.5932, 3.1602; 35, 1437.1952, 268.1670; 36, 1494.2651, 25.1211; 37, 1540.9879, 4.0357; 38, 1637.0773, 10.9180; 39, 1658.3213, 16.7066; 40, 1724.0023, 131.2034; 41, 2143.7847, 856.4489; 42, 3185.2343, 0.3111; 43, 3195.1714, 10.2846; 44, 3205.4122, 33.0931; 45, 3213.812, 16.3942; 46, 3229.8304, 6.6014; 47, 3233.2747, 4.2998; 48, 3493.7453, 37.6362;

\section{Calculation summary for 1,3 Migration of Ph TS2 iE (vacuum)}

Geometry optimisation and frequency calculation: Program version = x86-Linux-G03RevB.01. Method and basis set $=$ RB3LYP/6-31G(d). Calculated Energy = -477.0217468 (hartree), $-1994.9049451176\left(\mathrm{~kJ} \mathrm{~mol}^{-1}\right)$. Higher level single-point calculation: Program = x86-Linux-G03RevB.01. Method and basis set = RB3LYP/6311+G(3df,2p). Calculated Energy = -477.1903227 (hartree), $-1995.6099295314\left(\mathrm{~kJ} \mathrm{~mol}^{-1}\right)$. Structure definition (3D Coordinates: Atom, X coord/Å, Y coord/Å, Z coord/Å): C, 2.375427558, 0.1105122245, 
0.0377653698; C, 1.5275571322, -0.999649896, 0.0022355858; O, 1.5731247474, -2.2091847041, 0.0019285399; C, 1.4154403976, 1.1357699564, -0.0079685907; N, 1.0860820302, 2.3312094615, 0.0388448683; C, 0.0833163954, -0.1577330664, -0.0121723241; H, 3.4558197735, 0.1989192204, 0.0907883522; H, 1.8146161477, 3.0202342963, -0.2200734481; C, -0.6392996501, -0.1021025469, 1.2149986122; C, -2.0228748066, -0.015373268, 1.231077793; C, -2.7270044601, 0.0025316918, 0.0208852015; C, -2.0512154582, -0.0746035839, -1.2070674789; C, -0.6707868624, -0.1624460914, 1.2233840828; H, -0.0790084613, -0.1125260074, 2.1466283413; H, -2.5605665876, 0.0412099557, 2.1731526851; H, -3.8114728764, 0.0750086531, 0.0318047931; H, -2.6143489059, -0.0669893693, 2.1359629333; H, -0.1359727547, -0.2222778684, -2.1680725464. Harmonic Frequencies (Number, Frequency $\left(\mathrm{cm}^{-1}\right)$, IR Intensity $\left.\left(\mathrm{km} \mathrm{mol}^{-1}\right)\right): 1,-529.3789,300.5866 ; 2,82.0456,7.3199 ; 3,99.7146,3.5080 ; 4,126.2669$, 0.0692; 5, 193.0204, 3.1410; 6, 243.7122, 3.6349; 7, 298.6879, 93.5614; 8, 358.4032, 63.5510; 9, 393.1481, 0.7879; 10, 411.4207, 66.0834; 11, 559.8825, 17.4358; 12, 608.1623, 9.2518; 13, 616.5793, 0.5458; 14, 624.7697, 1.1138; 15, 672.589, 10.1691; 16, 684.7092, 47.7442; 17, 697.6744, 29.3823; 18, 751.3927, 54.8295; 19, 774.013, 9.4209; 20, 857.3714, 20.2136; 21, 864.2077, 152.9056; 22, 958.6108, 1.1180; 23, 985.7625, 0.1213; 24, 998.8822, 114.5233; 25, 1013.2166, 1.7171; 26, 1019.6373, 81.8580; 27, 1047.7489, 5.3184; 28, 1050.268, 20.3172; 29, 1111.6249, 2.0704; 30, 1196.6478, 2.7131; 31, 1212.0492, 35.6115; 32, 1215.4472 , 46.6212; 33, 1325.1286, 0.3407; 34, 1344.5451, 21.1983; 35, 1370.4114, 17.1532; 36, 1475.8313, 23.2397; 37, 1506.3688, 0.3498; 38, 1606.974, 0.3331; 39, 1637.0791, 46.0534; 40, 1847.0672, 1226.1355; 41, 1910.2801, 35.1172; 42, 3188.16, 0.2597; 43, 3192.7643, 0.2370; 44, 3199.154, 2.5925; 45, 3208.9744, 23.4549; 46, 3216.4832, 21.4657; 47, 3221.4939, 7.7306; 48, 3515.9014, 39.5157;

\section{Calculation summary for 1,3 Migration of Ph TS2 iZ (vacuum)}

Geometry optimisation and frequency calculation: Program version = x86-Linux-G03RevB.01. Method and basis set = RB3LYP/6-31G(d). Calculated Energy = -477.0288568 (hartree), -1994.9346791376 (kJ mol $\left.{ }^{-1}\right)$. Higher level single-point calculation: Program = x86-Linux-G03RevB.01. Method and basis set = RB3LYP/6-

311+G(3df,2p). Calculated Energy = -477.1973967 (hartree), -1995.6395129994 (kJ mol $\left.{ }^{-1}\right)$. Structure definition (3D Coordinates: Atom, X coord/Å, Y coord/Å, Z coord/Å): C, 2.4062808149, 0.066982702, 0.0001458946; C, 1.468598856, 1.1099687202, -0.0001442166; N, 1.3425556363, 2.3458341035, 0.0002070282; C, 1.5334413912, -1.0164456477, -0.0000436289; C, 0.0733865664, -0.1076206427, 0.0000004047; O, 1.5096773959, -2.2221410192, 0.0000168573; H, 3.4886401953, 0.122552022, 0.0002104804; H, 0.4423176022, 2.8061769929, -0.0001568843; C, -0.6638895326, -0.0920456256, 1.2174683268; C, -2.0500567736, -0.0392765895, 1.2175681837; C, -2.7439077396, -0.0103803712, 0.0001696757; C, -2.0502072344, -0.0393959236, -1.2173119085; C, -0.6640402577, -0.0921655047, 1.2173778126; H, -0.1175808228, -0.1168563326, 2.1574289618; H, -2.5991319501, -0.0234797527, 2.1549679385; H, -3.8299443301, 0.0309292299, 0.0002348671; H, -2.5993981943, -0.0236912028, 2.1546453697; H, -0.1178476652, -0.1170682308, -2.1574034723. Harmonic Frequencies (Number, Frequency $\left(\mathrm{cm}^{-1}\right)$, IR Intensity $\left.\left(\mathrm{km} \mathrm{mol}^{-1}\right)\right): 1,-503.064,337.6037 ; 2,93.4532,7.1142 ; 3,98.3647,2.9809 ; 4,116.3269$, 0.9313; 5, 194.8512, 0.0121; 6, 229.9668, 2.6208; 7, 232.46, 87.7943; 8, 355.6325, 14.6522; 9, 398.7268, 1.5407; 10, 399.3189, 80.0091; 11, 566.2913, 4.3395; 12, 606.4553, 12.4974; 13, 613.0733, 1.7473; 14 , 623.1842, 2.3579; 15, 669.5004, 2.6106; 16, 676.508, 47.4064; 17, 695.3534, 49.3328; 18, 758.9152, 44.4765; 19, 761.5943, 22.2078; 20, 863.2715, 0.0600; 21, 912.5277, 483.0376; 22, 955.9486, 31.0317; 23, 986.8491, 0.0002; 24, 990.5935, 121.9895; 25, 1011.3485, 2.0576; 26, 1013.1986, 18.6064; 27, 1036.2648, 26.2200; 28, 1050.1565, 15.3350; 29, 1109.0379, 2.1612; 30, 1195.4146, 1.4899; 31, 1211.664, 23.1029; 32, 1218.0026, 106.8455; 33, 1322.1852, 0.3841; 34, 1351.7437, 14.5173; 35, 1364.4519, 9.9096; 36, 1475.992, 17.4087; 37 , 1507.4934, 1.3992; 38, 1607.9816, 0.0054; 39, 1633.9669, 25.5342; 40, 1864.5899, 1008.2312; 41, 1925.0468, 37.7688; 42, 3185.9238, 0.7222; 43, 3189.4174, 0.3506; 44, 3197.2567, 1.3123; 45, 3206.1382, 23.8554; 46, 3214.9237, 20.7270; 47, 3240.8957, 5.0918; 48, 3627.6127, 60.9436; 
Geometry optimisation and frequency calculation: Program version = x86-Linux-G03RevB.01. Method and basis set = RB3LYP/6-31G(d). Calculated Energy = -477.1019208 (hartree), -1995.2402327856 (kJ mol $\left.{ }^{-1}\right)$. Higher level single-point calculation: Program = x86-Linux-G03RevB.01. Method and basis set $=$ RB3LYP/6-

$311+G(3 d f, 2 p)$. Calculated Energy = -477.2703945 (hartree), -1995.944789799 (kJ mol $\left.{ }^{-1}\right)$. Structure definition

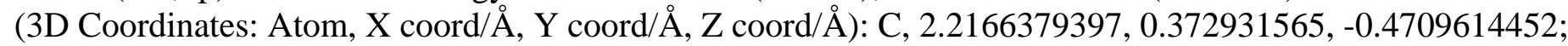
C, 2.362633749, 0.3672966498, 0.8475659381; O, 2.5248615435, 0.3628297952, 2.0034765955; C, 0.9249856058, 0.3773877761, -1.1907878234; N, 0.8082288428, 0.721556314, -2.4235129447; C, 0.3121152962, -0.0189699058, -0.4518986677; H, 3.1375427101, 0.4802130417, -1.038190928; H, 1.7111649838, 1.0058867254, -2.8155154688; C, -1.5310164101, 0.5830447809, -0.8022154378; C, 2.7058239986, 0.2361176301, -0.1413309458; C, -2.685063785, -0.7265336759, 0.8723266336; C, 1.4824514452, -1.3435364251, 1.2154643607; C, -0.3022818183, -0.9922926607, 0.55772606; H, 1.5331615453, 1.3224277218, -1.5958771505; H, -3.6407404015, 0.7176821057, -0.4152276452; H, 3.6028822854, -0.9965426455, 1.3878032814; H, -1.4601780042, -2.1057368885, 1.9895091785; H, 0.6187330478, -1.5101370273, 0.8089445469. Harmonic Frequencies (Number, Frequency ( $\mathrm{cm}^{-1}$ ), IR Intensity $\left(\mathrm{km} \mathrm{mol}^{-1}\right)$ ): 1, 51.3871, 0.3953; 2, 89.1236, 2.2760; 3, 113.4728, 2.8740; 4, 175.5086, 3.2474; 5, 223.8879, 4.5630; 6, 348.3278, 0.2048; 7, 395.6732, 11.6062; 8, 421.0354, 0.1749; 9, 495.9203, 11.3831; 10, 520.7404, 1.3529; 11, 531.4079, 11.2632; 12, 556.732, 19.0671; 13, 633.4089, 0.3641; 14, 663.4506, 4.0166; 15, 687.83, 9.9408; 16, 716.1219, 47.6122; 17, 755.0877, 1.2069; 18, 798.5717, 6.2744; 19, 864.7221, 3.0871; 20, 893.0433, 89.0931; 21, 944.9303, 9.0507; 22, 977.731, 2.1180; 23, 1002.2329, 0.2841; 24, 1017.7013, 2.4851; 25, 1037.7427, 44.4573; 26, 1065.0528, 5.3620; 27, 1115.639, 5.8550; 28, 1153.018, 4.6145; 29, 1193.1285, 25.1811; 30, 1196.249, 36.5662; 31, 1216.212, 7.1814; 32, 1339.6747, 12.9798; 33, 1366.6702, 0.9010; 34, 1388.5588, 108.5300; 35, 1396.9866, 101.7641; 36, 1493.5956, 15.0628; 37, 1543.7492, 7.9860; 38, 1633.6842, 27.0783; 39, 1661.7281, 7.1281; 40, 1667.5647, 126.9529; 41, 2218.6178, 715.6163; 42, 3184.4955, 0.4459; 43, 3193.5918, 8.7042; 44, 3195.1479, 3.0766; 45, 3202.8278, 28.7284; 46, 3212.0006, 17.2519; 47, 3229.023, 3.9904; 48, 3430.0913, 8.5085;

\section{Calculation summary for 1,3 Migration of Ph 1 sE iZ (vacuum)}

Geometry optimisation and frequency calculation: Program version = x86-Linux-G03RevB.01. Method and basis set = RB3LYP/6-31G(d). Calculated Energy = -477.1023424 (hartree), -1995.2419959168 (kJ mol $\left.{ }^{-1}\right)$. Higher level single-point calculation: Program = x86-Linux-G03RevB.01. Method and basis set = RB3LYP/6311+G(3df,2p). Calculated Energy = -477.2711776 (hartree), -1995.9480647232 (kJ mol $\left.{ }^{-1}\right)$. Structure definition (3D Coordinates: Atom, X coord/Å, Y coord/A, Z coord/Å): C, 2.2244609076, 0.0995208151, 0.5924153011; C, 0.9340352503, -0.1161092818, -1.2608208534; N, 0.9533829804, -0.2678176439, 2.5379254804; C, 2.3851231601, 0.4771986834, 0.6680163344; C, -0.3089204446, -0.1362514056, 0.4245255976; O, 2.5671904952, 0.7998497854, 1.7756287063; H, 3.1243353897, 0.0226715148, 1.1957299059; H, 0.0036752115, -0.4432236314, -2.8805492815; C, -1.4499492426, 0.551627693, 0.8659749005; C, -2.6264405471, 0.5264604288, -0.1196804311; C, -2.6830079563, -0.1949490374, 1.0752399326; C, -1.5562697228, -0.8877861245, 1.519994291; C, -0.3746170252, -0.8552337215, 0.7786152262; H, -1.4012818456, 1.1265368877, -1.7869919496; H, -3.4975045865, 1.0752509914, 0.4671986291; H, -3.600712963, -0.2159254724, 1.6565729512; H, -1.5963919709, -1.4593406391, 2.443180049; H, 0.4901896646, -1.416912724, 1.1204732754. Harmonic Frequencies (Number, Frequency $\left(\mathrm{cm}^{-1}\right)$, IR Intensity $\left.\left(\mathrm{km} \mathrm{mol}^{-1}\right)\right): 1,52.5324,0.3593 ; 2,84.7853,0.1904 ; 3,119.4407,1.5202 ; 4,171.7556$, 1.5903; 5, 214.6896, 2.5707; 6, 338.8367, 2.8084; 7, 382.1123, 5.5026; 8, 418.9864, 0.0990; 9, 509.1501, 6.0686; 10, 523.1089, 6.3790; 11, 537.5227, 11.2625; 12, 566.6158, 26.3850; 13, 632.6533, 0.8414; 14, 663.1889, 14.8075; 15, 713.1699, 7.1212; 16, 725.1521, 71.0136; 17, 751.4972, 8.0424; 18, 793.1404, 8.3054; 
19, 865.5711, 1.3875; 20, 897.4134, 37.1904; 21, 943.908, 2.5569; 22, 975.7833, 1.0043; 23, 1001.938, 0.3812; 24, 1016.9301, 1.8796; 25, 1035.096, 65.0982; 26, 1060.9936, 16.2077; 27, 1114.9342, 2.0598; 28, 1130.3135, 3.6552; 29, 1195.1732, 0.0013; 30, 1214.1935, 3.5737; 31, 1218.3477, 55.7029; 32, 1335.7682, 4.8018; 33, 1364.9559, 13.8648; 34, 1368.5723, 38.9731; 35, 1428.9456, 95.6153; 36, 1491.6662, 9.8280; 37, 1541.9292 , 1.9874; 38, 1631.5634, 39.7951; 39, 1659.0714, 60.9073; 40, 1662.4254, 81.2801; 41, 2220.742, 734.9483; 42, 3185.6463, 1.1279; 43, 3192.0183, 0.4839; 44, 3199.5367, 12.6341; 45, 3206.8178, 26.5841; 46, 3209.5382, 11.7254; 47, 3214.7628, 14.5813; 48, 3435.7532, 1.4135;

\section{Calculation summary for 1,3 Migration of Ph $1 \mathrm{sZ}$ iE (vacuum)}

Geometry optimisation and frequency calculation: Program version $=$ x86-Linux-G03RevB.01. Method and basis set = RB3LYP/6-31G(d). Calculated Energy = -477.1007469 (hartree), -1995.2353235358 $\left(\mathrm{kJ} \mathrm{mol}^{-1}\right)$. Higher level single-point calculation: Program = x86-Linux-G03RevB.01. Method and basis set = RB3LYP/6311+G(3df,2p). Calculated Energy = -477.2689216 (hartree), -1995.9386301312 (kJ mol $\left.{ }^{-1}\right)$. Structure definition (3D Coordinates: Atom, X coord/Å, Y coord/A, Z coord/Å): C, 0.8486281968, -0.3110791751, 1.7362128288; C, 0.8610772167, -0.1337661201, 3.0482296342; H, 1.8066705302, -0.5213390022, 1.2730008891; O, 0.8582014474, 0.0352510689, 4.203249165; C, -0.3983667339, -0.2549578747, 0.9326968827; N, -1.5952006309, -0.3916456833, 1.382580781; C, -0.2204583578, -0.028293037, 0.5339302273; H, -1.6031227776, -0.5941793578, 2.3868758893; C, -1.2594174347, -0.3917024628, 1.4067819899; C, -1.1337472898, -0.1909121878, -2.7778977844; C, 0.0286151017, 0.382046713, 3.3027628252; C, 1.0621517867, 0.756080871, -2.4444546568; C, 0.9390281708, 0.5504468908, 1.0695338725; H, -2.1561712382, -0.8319859069, -0.9848704855; H, -1.9430142852, -0.4841606719, 3.441334335; H, 0.1257555478, 0.537094532, -4.3740219676; H, 1.9642390244, 1.2135727267, 2.8416238814; H, 1.7413720963, 0.8733272072, -0.4127528334. Harmonic Frequencies (Number, Frequency

$\left(\mathrm{cm}^{-1}\right)$, IR Intensity $\left.\left(\mathrm{km} \mathrm{mol}^{-1}\right)\right): 1,39.5154,0.1325 ; 2,66.6971,2.8636 ; 3,113.9661,1.8117 ; 4,168.0058$, 3.8369; 5, 234.8651, 4.4720; 6, 329.7028, 1.4012; 7, 417.2255, 6.5790; 8, 418.46, 2.3320; 9, 486.501, 11.0152; 10, 502.7004, 3.0036; 11, 535.3023, 3.9362; 12, 562.8061, 15.8626; 13, 633.757, 0.1719; 14, 677.9204, 37.5933; 15, 693.3074, 4.4509; 16, 714.4618, 53.1777; 17, 797.8445, 9.2216; 18, 842.4444, 20.1725; 19, 863.025, 4.8243; 20, 882.5947, 69.0547; 21, 944.9021, 7.6628; 22, 979.2069, 0.9784; 23, 987.2195, 55.8313; 24, 1005.2168, 2.7549; 25, 1018.0162, 0.7230; 26, 1060.375, 0.2219; 27, 1114.4573, 2.8635; 28, 1147.5081, 4.3045; 29, 1188.6845, 50.3671; 30, 1196.0078, 18.9756; 31, 1218.6177, 2.5841; 32, 1319.0285, 28.3790; 33, 1354.5918, 6.9349; 34, 1367.3536, 0.5246; 35, 1435.8943, 214.3942; 36, 1494.768, 27.6949; 37, 1543.1348, 4.4705; 38, 1632.3206, 23.4394; 39, 1659.6447, 18.9602; 40, 1665.2619, 42.8551; 41, 2218.9816, 934.2790; 42, 3183.653, 0.6249; 43, 3192.6544, 7.0697; 44, 3201.9313, 32.3053; 45, 3211.1331, 23.5765; 46, 3219.9466, 9.7961; 47, 3232.714, 3.6830; 48, 3429.4642, 7.3286;

\section{Calculation summary for 1,3 Migration of $\mathrm{Ph} 1 \mathrm{sZ}$ iZ (vacuum)}

Geometry optimisation and frequency calculation: Program version = x86-Linux-G03RevB.01. Method and basis set = RB3LYP/6-31G(d). Calculated Energy = -477.1027502 (hartree), -1995.2437013364 $\left(\mathrm{kJ} \mathrm{mol}^{-1}\right)$. Higher level single-point calculation: Program = x86-Linux-G03RevB.01. Method and basis set = RB3LYP/6-

$311+G(3 d f, 2 p)$. Calculated Energy = -477.2714438 (hartree), -1995.9491779716 ( $\left.\mathrm{kJ} \mathrm{mol}^{-1}\right)$. Structure definition (3D Coordinates: Atom, X coord/Å, Y coord/A, Z coord/Å): C, 0.839689843, -0.3286444445, 1.7345346934; C, 0.8249356555, -0.2588849601, 3.0617198309; H, 1.7602128281, -0.6596917049, 1.2685662337; O, 0.8412686912, -0.1932848522, 4.2236243579; C, -0.3742603542, -0.033525813, 0.9588851805; N, -1.4832139981, 0.150559879, 1.5872162034; C, -0.212349541, 0.0280501007, 0.5279484138; H, -2.2275447396, 0.4028726767, 0.9315623992; C, -1.2429967379, -0.4356108115, 1.3602570797; C, -1.1254652404, -0.359502582, -2.7466131089; C, 0.0227852551, 0.1875415218, 3.3237693872; C, 1.0545250665, 0.6513526725, -2.5059165009; C, 0.9415563576, 0.5646790756, - 
1.118464645; H, -2.1284194471, -0.8816939304, -0.9150714816; H, -1.9271010384, -0.7345684415, 3.3770166186; H, 0.1141902984, 0.2480764352, -4.404781702; H, 1.9483901524, 1.0832189658, 2.9478523862; H, 1.7420985793, 0.9414171058, -0.4879381473. Harmonic Frequencies (Number, Frequency

$\left(\mathrm{cm}^{-1}\right)$, IR Intensity $\left.\left(\mathrm{km} \mathrm{mol}^{-1}\right)\right): 1,50.1195,0.1460 ; 2$, 85.543, 0.8992; 3, 101.1061, 0.9403; 4, 171.1809, 0.1108; 5, 227.4996, 1.0405; 6, 310.3767, 3.0196; 7, 397.8996, 2.6053; 8, 418.0477, 0.0769; 9, 482.637, 9.1447; 10, 512.5098, 7.4152; 11, 545.6197, 0.7124; 12, 564.3629, 33.2136; 13, 632.6577, 0.3285; 14, 677.5971, 26.1763; 15, 699.9398, 11.2597; 16, 717.6206, 46.3578; 17, 786.3518, 28.1668; 18, 857.3868, 21.2215; 19, 864.9818, 1.7243; 20, 877.1291, 45.1844; 21, 942.8325, 3.0861; 22, 975.6624, 0.7410; 23, 1001.405, 2.5492; 24, 1011.7194, 30.6382; 25, 1017.545, 0.9295; 26, 1059.0288, 3.4276; 27, 1116.0558, 1.0748; 28, 1148.9557, 1.0719; 29, 1194.9097, 0.0205; 30, 1214.9958, 33.3592; 31, 1216.2373, 6.2759; 32, 1307.1815, 87.9239; 33, 1346.0997, 9.5188; 34, 1368.0702, 3.3698; 35, 1466.0799, 217.3241; 36, 1493.9585, 41.4969; 37, 1543.2956, 1.5133; 38, 1630.7031, 32.0657; 39, 1657.2548, 35.0991; 40, 1663.0189, 30.4696; 41, 2229.7508, 959.5083; 42, 3184.8574, 1.6894; 43, 3190.8991, 0.3979; 44, 3199.0892, 12.2807; 45, 3206.1458, 30.4775; 46, 3214.241, 16.2935; 47, 3234.8325, 7.5944; 48, 3449.6671, 1.0205;

\section{Calculation summary for 1,3 Migration of $\mathrm{Ph} 3 \mathrm{sE}$ (acetonitrile)}

Geometry optimisation and frequency calculation: Program version = DEC-AXP-OSF/1-G03RevB.05. Method and basis set = RB3LYP/6-31G(d). Calculated Energy = -477.1180372 (hartree), $-1995.3076315704\left(\mathrm{~kJ} \mathrm{~mol}^{-1}\right)$. Higher level single-point calculation: Program = DEC-AXP-OSF/1-G03RevB.05. Method and basis set =

RB3LYP/6-311++G(3df,2p). Calculated Energy = -477.2881164 (hartree), -1996.0189027848 (kJ mol-1). Structure definition (3D Coordinates: Atom, X coord/A, Y coord/Å, Z coord/A): C, -2.2643841171, 0.4555649334, -0.1642752666; C, -2.4374683528, -0.7993655113, 0.2448314513; N, -2.6241316704, 1.9702108408, 0.5100589785; C, -0.9868929721, 1.1778807721, -0.2099533211; C, 0.3122114028, 0.4351100879, -0.1199279699; O, -0.990249035, 2.4092247591, -0.3067588561; H, -3.1608156652, 1.0447821944, -0.3597972753; H, -2.7631195491, -2.2566425057, 1.4978554272; C, 1.3954365754, 1.0757631656, 0.5040608256; C, 2.6307777244, 0.4394661467, 0.6018095173; C, 2.8059476697, 0.8356915139, 0.0530285113; C, 1.741283872, -1.4690801916, -0.5925588863; C, 0.4972635273, 0.8403625936, -0.6755991424; H, 1.2516097478, 2.0716670198, 0.9168553621; H, 3.4598695039, 0.9377325663, 1.1018904947; H, 3.7732550246, -1.3311887256, 0.1228878512; H, 1.8792538531, 2.4526743722, -1.0386765975; H, -0.3141909198, -1.3317101359, -1.2058515774. Harmonic Frequencies (Number, Frequency ( $\left.\mathrm{cm}^{-1}\right)$, IR Intensity $\left(\mathrm{km} \mathrm{mol}^{-1}\right)$ ): 1, 24.2167, 1.8295; 2, 86.4702, 4.2926; 3, 113.4676, 5.2276; 4, 172.5876, 4.5473; 5, 212.0426, 1.9393; 6, 343.0157, 6.3799; 7, 393.4908, 20.6237; 8, 419.0555, 1.2999; 9, 460.5434, 74.3471; 10, 490.1804, 10.5167; 11, 522.2595, 50.3254; 12, 557.0641, 26.1415; 13, 623.8618, 3.3942; 14, 655.1006, 37.6139; 15, 708.683, 18.0467; 16, 733.9782, 110.1606; 17, 744.9003, 25.3229; 18, 815.6551, 2.0511; 19, 832.7838, 144.5401; 20, 865.658, 4.3192; 21, 880.502, 826.9592; 22, 949.5951, 2.1830; 23, 982.4618, 2.1499; 24, 1009.0132, 0.2105; 25, 1009.356, 3.6301; 26, 1044.3607, 17.5079; 27, 1082.3019, 73.9706; 28, 1110.3459, 3.3139; 29, 1147.9167, 3.6597; 30, 1182.279, 0.1418; 31, 1201.7075, 10.3941; 32, 1297.3798, 371.7225; 33, 1341.4507, 9.1991; 34, 1361.8848, 7.1517; 35, 1403.0728, 119.5471; 36, 1485.9068, 30.5413; 37, 1534.762, 2.8696; 38, 1628.15, 84.4018; 39, 1650.3643, 82.6048; 40, 1674.2784, 714.1769; 41, 2117.7109, 1035.9340; 42, 3129.4937, 36.8825; 43, 3146.0857, 0.2447; 44, 3155.1834, 2.8899; 45, 3164.0354, 6.3419; 46, 3169.4099, 443.0719; 47, 3175.8782, 2.3794; 48, 3187.4433, 1.4119;

\section{Calculation summary for 1,3 Migration of $\mathrm{Ph} 3 \mathrm{sZ}$ (acetonitrile)}

Geometry optimisation and frequency calculation: Program version = DEC-AXP-OSF/1-G03RevB.05. Method and basis set = RB3LYP/6-31G(d). Calculated Energy = -477.1201705 (hartree), $-1995.316553031\left(\mathrm{~kJ} \mathrm{~mol}^{-1}\right)$. Higher level single-point calculation: Program = DEC-AXP-OSF/1-G03RevB.05. Method and basis set = RB3LYP/6-311++G(3df,2p). Calculated Energy = -477.2898524 (hartree), -1996.0261627368 (kJ mol-1). 
Structure definition (3D Coordinates: Atom, X coord/A, Y coord/Å, Z coord/Å): C, 1.8479801294, 0.6882629108, -0.1406161962; C, 3.1588114857, -0.4614888413, -0.0579583078; H, 1.5282864842, 1.706176004, -0.3464058626; N, 4.3485069819, -0.2333075542, -0.102738761; C, 0.9031151065, 0.4193475771, 0.0401391195; O, 1.2943155686, 1.5822749865, 0.199774884; C, -0.5650178381, 0.1128944366, 0.0112482591; H, 4.9264353223, -0.2890651605, 0.7572247872; C, -1.4563499498, 1.1948656222, -0.0910249201; C, -2.8320860275, 0.9814665196, -0.1176330288; C, -3.3399667527, 0.3192811024, -0.0309836729; C, -2.4640392522, -1.4018407014, 0.0813198552; C, -1.0847417441, 1.1890749855, 0.0987246981; H, -1.0493816524, 2.2006867104, -0.1527164554; H, -3.5109310482, 1.8283830617, -0.205130499; H, -4.4158010564, -0.4877876107, -0.0492609513; H, -2.8539030545, 2.4155623072, 0.1570992188; H, -0.4250093606, -2.0472793869, 0.2008671807. Harmonic Frequencies

(Number, Frequency $\left(\mathrm{cm}^{-1}\right)$, IR Intensity $\left(\mathrm{km} \mathrm{mol}^{-1}\right)$ ): 1, -50.7844, 3.5639; 2, 43.571, 10.2891; 3, 108.324, 1.7446; 4, 171.125, 2.1549; 5, 227.8168, 11.3282; 6, 328.6091, 3.8855; 7, 412.6619, 1.3671; 8, 431.4649, 11.6600; 9, 468.8565, 85.8245; 10, 470.9534, 6.1549; 11, 480.2699, 1.6895; 12, 538.6853, 58.8051; 13, 624.0306, 1.4887; 14, 684.0767, 42.0482; 15, 699.3185, 7.0255; 16, 727.1381, 138.8837; 17, 809.2909, 71.3043; 18, 827.989, 36.0395; 19, 860.7184, 1061.4712; 20, 861.7611, 2.5083; 21, 870.7916, 41.9746; 22 , 949.7357, 1.7296; 23, 985.0825, 0.3347; 24, 1008.3988, 17.3111; 25, 1012.1189, 0.7885; 26, 1022.9487, 113.9424; 27, 1054.9159, 10.8434; 28, 1109.6365, 7.4425; 29, 1162.4588, 17.7639; 30, 1181.3859, 6.5377; 31, 1210.4212, 21.9824; 32, 1237.7531, 320.4045; 33, 1346.3232, 4.0704; 34, 1364.7449, 10.3865; 35, 1436.2303, 547.6453; 36, 1487.0762, 58.4176; 37, 1536.5667, 8.2811; 38, 1625.8808, 80.6312; 39, 1648.8489, 61.2729; 40, 1671.0925, 249.8851; 41, 2122.4073, 1507.9287; 42, 3146.4448, 0.2913; 43, 3155.9893, 2.6118; 44, 3164.2137, 2.6783; 45, 3169.422, 329.8985; 46, 3173.8622, 223.0554; 47, 3183.6063, 4.2205; 48, 3187.4806, 1.5040;

\section{Calculation summary for 1,3 Migration of Ph TS2 iE (acetonitrile)}

Geometry optimisation and frequency calculation: Program version = DEC-AXP-OSF/1-G03RevB.05. Method and basis set = RB3LYP/6-31G(d). Calculated Energy = -477.0431205 (hartree), -1994.994329931 (kJ mol ${ }^{-1}$ ). Higher level single-point calculation: Program = DEC-AXP-OSF/1-G03RevB.05. Method and basis set = RB3LYP/6-311++G(3df,2p). Calculated Energy = -477.2137193 (hartree), -1995.7077741126 (kJ mol ${ }^{-1}$ ). Structure definition (3D Coordinates: Atom, X coord/Å, Y coord/Å, Z coord/Å): C, 2.355197338, 0.1280528824, -0.0145712895; C, 1.523269408, -0.9898155917, 0.01908328; O, 1.6140858672, -2.212013371, 0.0514651754; C, 1.3528690075, 1.125203114, -0.0364914386; N, 1.0211092984, 2.3259883686, 0.0664958868; C, 0.1002041068, -0.1846163736, 0.0041785492; H, 3.4362738735, 0.2495170016, 0.0224653096; H, 1.817703453, 2.9857788086, -0.0884859948; C, -0.6359938402, -0.1193233661, 1.2328666515; C, -2.0138277757, 0.0004707306, 1.2342208873; C, -2.7058021029, 0.0308834773, 0.0121083331; C, -2.0258407954, -0.0665239671, -1.2132760473; C, -0.6480063677, -0.1861207792, 1.2189757109; H, -0.0830536561, -0.1413099308, 2.1723983229; H, -2.5634621106, 0.0697773962, 2.1712762582; H, -3.7912848762, 0.1289268251, 0.0147471044; H, -2.5847012777, -0.0484024671, 2.1472589798; H, -0.1043413034, -0.2593600059, -2.1613208859. Harmonic Frequencies (Number, Frequency $\left(\mathrm{cm}^{-1}\right)$, IR Intensity $\left.\left(\mathrm{km} \mathrm{mol}^{-1}\right)\right): 1,-427.0086,684.8546 ; 2,60.8012,16.8895 ; 3,97.2544,1.0903 ; 4$, 130.9923, 0.1103; 5, 196.6913, 5.4034; 6, 251.4624, 2.8955; 7, 348.9232, 55.8587; 8, 352.6851, 121.1417; 9, 389.2768, 3.4726; 10, 409.9676, 113.5877; 11, 561.9278, 38.8958; 12, 605.028, 0.3284; 13, 612.2342, 2.1766; 14, 630.7534, 3.7427; 15, 678.5084, 101.7573; 16, 682.1731, 26.5282; 17, 709.7308, 49.8723; 18, 749.3574, 92.8701; 19, 780.008, 31.2645; 20, 859.2205, 0.0873; 21, 895.6919, 269.5685; 22, 977.7622, 1.4397; 23, 990.2567, 166.0850; 24, 998.397, 0.2035; 25, 1022.562, 324.0975; 26, 1029.3108, 0.7540; 27, 1040.3412, 48.7665; 28, 1051.5797, 27.6164; 29, 1107.2643, 2.6548; 30, 1185.5506, 7.0598; 31, 1201.8808, 12.5918; 32, 1221.8487, 164.6917; 33, 1319.6378, 0.0042; 34, 1327.6986, 96.3405; 35, 1375.0001, 54.0013; 36, 1466.7321, 83.4872; 37, 1496.4442, 5.9718; 38, 1593.926, 0.0008; 39, 1629.2602, 182.2015; 40, 1742.2021, 2651.8809; 41, 1846.1569, 103.9529; 42, 3139.3851, 0.3903; 43, 3140.3948, 0.7091; 44, 3148.139, 2.0136; 45, 3162.8754, 
0.0073; 46, 3166.6586, 0.2620; 47, 3173.3539, 0.4026; 48, 3224.3154, 375.2073;

\section{Calculation summary for 1,3 Migration of $\mathrm{Ph}$ TS2 iZ (acetonitrile)}

Geometry optimisation and frequency calculation: Program version = x86-Linux-G03RevB.01. Method and basis set $=$ RB3LYP/6-31G(d). Calculated Energy = -477.0492489 (hartree), -1995.0199588998 $\left(\mathrm{kJ} \mathrm{mol}^{-1}\right)$. Higher level single-point calculation: Program = x86-Linux-G03RevB.01. Method and basis set $=$ RB3LYP/6311++G(3df,2p). Calculated Energy = -477.2194076 (hartree), -1995.7315625832 (kJ mol $\left.{ }^{-1}\right)$. Structure definition (3D Coordinates: Atom, X coord/Å, Y coord/Å, Z coord/Å): C, 2.3862689543, 0.0646124632, 0.0004117205; C, 1.4188051755, 1.0925051183, -0.0000835645; N, 1.3164209262, 2.3387789853, 0.0010048075; C, 1.51009492, -1.0144739483, -0.0000415884; C, 0.0954748302, -0.1046106698, 0.000222466; O, 1.5113834416, -2.2341806169, 0.000177513; H, 3.4715371051, 0.1281609723, 0.0009466272; H, 0.3932057446, 2.7838400541, 0.0009943161; C, -0.6471446736, -0.0900090118, 1.2248030086; C, -2.0302203195, -0.0307119156, 1.2227411813; C, -2.7207027128, 0.0017820945, 0.0003237519; C, -2.0306544914, -0.0310723242, -1.2223817104; C, -0.6476020995, -0.090206568, 1.2250212583; H, -0.0988391719, -0.1136099341, 2.1670633902; H, -2.5839270343, -0.0099361099, 2.1598452799; H, -3.8093006877, 0.0509157038, 0.0004934505; H, -2.5847328004, -0.0104315155, 2.1592744248; H, -0.0998746713, -0.1138385634, -2.1676295381. Harmonic Frequencies (Number, Frequency $\left(\mathrm{cm}^{-1}\right)$, IR Intensity $\left.\left(\mathrm{km} \mathrm{mol}^{-1}\right)\right): 1,-446.6902,680.8772 ; 2,106.1959,7.3420 ; 3,108.2493,1.3183 ; 4$, 126.4366, 0.0265; 5, 200.7947, 2.7473; 6, 239.9622, 0.3626; 7, 355.2672, 41.1224; 8, 389.7229, 9.4951; 9, 410.8583, 193.9829; 10, 430.1992, 158.4159; 11, 571.315, 5.6579; 12, 605.2448, 0.1515; 13, 611.2081, 5.7687; 14, 631.1771, 11.6006; 15, 675.9311, 4.3404; 16, 682.2216, 140.6479; 17, 693.3625, 91.8240; 18, 755.8475, 73.1626; 19, 772.0051, 43.9135; 20, 860.8558, 0.1099; 21, 944.522, 1093.9319; 22, 972.0204, 51.1571; 23, 992.1628, 207.3252; 24, 997.8458, 0.0146; 25, 1004.1908, 102.2317; 26, 1025.6799, 30.9112; 27, 1037.1736, 131.2638; 28, 1041.4404, 38.7710; 29, 1106.0368, 2.6531; 30, 1184.7795, 4.7971; 31, 1201.8496, 12.1612; 32, 1224.215, 240.6508; 33, 1317.8301, 0.0478; 34, 1334.6676, 46.1788; 35, 1369.3811, 38.9315; 36, 1467.2359, 69.2340; 37, 1497.4913, 9.2658; 38, 1594.2156, 0.0793; 39, 1628.0153, 108.7026; 40, 1766.5083, 2245.3963; 41, 1861.0479, 8.1336; 42, 3138.0692, 0.3399; 43, 3138.9545, 0.4932; 44, 3148.734, 1.7355; 45, 3161.6449, 0.0244; 46, 3166.0735, 0.3945; 47, 3182.1745, 0.2937; 48, 3355.6059, 271.0457;

\section{Calculation summary for 1,3 Migration of $\mathrm{Ph} 1 \mathrm{sE}$ iE (acetonitrile)}

Geometry optimisation and frequency calculation: Program version = x86-Linux-G03RevB.01. Method and basis set = RB3LYP/6-31G(d). Calculated Energy = -477.1161484 (hartree), -1995.2997326088 (kJ mol $\left.{ }^{-1}\right)$. Higher level single-point calculation: Program = x86-Linux-G03RevB.01. Method and basis set = RB3LYP/6-

311++G(3df,2p). Calculated Energy = -477.2858751 (hartree), -1996.0095296682 (kJ mol $\left.{ }^{-1}\right)$. Structure definition (3D Coordinates: Atom, X coord/Å, Y coord/A, Z coord/Å): C, 2.2109943879, 0.4119440062, 0.4636356239; C, 2.3235500928, 0.4191730918, 0.8572413254; O, 2.4529598457, 0.430677024, 2.0182231709; C, 0.9380661953, 0.3509101118, -1.204027368; N, 0.8405258588, 0.6255208104, 2.4584246906; C, -0.3025728886, -0.0320246328, -0.4602179403; H, 3.1455056772, 0.5504083993, 1.0113870905; H, 1.7562866456, 0.9050040944, -2.8442065914; C, -1.5146342217, 0.6031925937, 0.7758974953; C, -2.688221094, 0.2628441742, -0.1055194301; C, -2.6713414049, -0.7273499714, 0.8826774891; C, -1.4744697929, -1.3758049509, 1.1928362651; C, -0.295773116, -1.0295541264, 0.5275707792; H, -1.5207557083, 1.3714928951, -1.5450291438; H, -3.6185083303, 0.7727105111, 0.3516387889; H, -3.5886766153, -0.9936041679, 1.405652031; H, -1.4556439032, -2.1593719015, 1.9486399559; H, 0.6208435096, -1.5706834719, 0.7549890867. Harmonic Frequencies (Number, Frequency

$\left(\mathrm{cm}^{-1}\right)$, IR Intensity $\left.\left(\mathrm{km} \mathrm{mol}^{-1}\right)\right): 1,53.1417,1.1458 ; 2,89.8982,2.6468 ; 3,108.8796,7.2884 ; 4,173.1908$, 5.4151; 5, 216.9427, 5.6723; 6, 340.7454, 0.5104; 7, 385.2493, 15.0146; 8, 421.5203, 0.4303; 9, 502.7037, 19.0274; 10, 524.6885, 9.2715; 11, 532.5422, 14.3815; 12, 557.7452, 29.0816; 13, 625.5094, 0.3758; 14, 
661.3019, 6.0373; 15, 694.5758, 15.9616; 16, 717.0284, 75.6311; 17, 749.026, 1.8881; 18, 798.6712, 11.6418; 19, 867.1535, 3.5815; 20, 884.2909, 156.9005; 21, 948.5671, 12.1709; 22, 982.5732, 2.8046; 23, 1006.9016, 0.5486; 24, 1008.8731, 3.4554; 25, 1037.3881, 41.2856; 26, 1062.8666, 20.1864; 27, 1109.2575, 9.9549; 28, 1140.1838, 7.4787; 29, 1181.6288, 0.6089; 30, 1198.9139, 56.2680; 31, 1209.2021, 65.2224; 32, 1333.9672, 24.7226; 33, 1361.3476, 2.4830; 34, 1385.5696, 217.2969; 35, 1396.1315, 215.7379; 36, 1486.1283, 34.4492; 37, 1538.1424, 25.3923; 38, 1624.6163, 120.7013; 39, 1648.3965, 289.9712; 40, 1654.2865, 4.8535; 41, 2180.0317, 1473.7826; 42, 3107.4293, 55.9943; 43, 3145.2945, 0.2386; 44, 3153.1268, 2.6939; 45, 3160.9936, 6.1321; 46, 3168.163, 2.4895; 47, 3181.2087, 1.0942; 48, 3274.4529, 34.3638;

\section{Calculation summary for 1,3 Migration of Ph 1 sE iZ (acetonitrile)}

Geometry optimisation and frequency calculation: Program version = x86-Linux-G03RevB.01. Method and basis set = RB3LYP/6-31G(d). Calculated Energy = -477.1155073 (hartree), -1995.2970515286 (kJ mol-1 $)$. Higher level single-point calculation: Program = x86-Linux-G03RevB.01. Method and basis set = RB3LYP/6-

311++G(3df,2p). Calculated Energy = -477.285665 (hartree), -1996.00865103 $\left(\mathrm{kJ} \mathrm{mol}^{-1}\right)$. Structure definition (3D Coordinates: Atom, X coord/A, Y coord/Å, Z coord/Å): C, -2.2829570342, 0.2903408805, 0.0765208513; C, -1.08629905, 1.128135363, 0.2228751891; N, -1.2632477216, 2.3912747061, 0.4088322951; C, 2.281884829, -0.974288706, -0.3204810729; C, 0.2546428745, 0.4663912092, 0.1312288886; O, 2.3206530236, -2.090503689, -0.6633930593; H, -3.26469852, 0.7407407721, 0.2297360438; H, 0.3559202729, 2.862950065, 0.5296010781; C, 1.2655165404, 1.0542475137, -0.6463919346; C, 2.5252079091, 0.4622050151, -0.7339391667; C, 2.7949898105, -0.7197258853, -0.0373137822; C, 1.7982806195, -1.3071282911, 0.7453616429; C, 0.5327506538, -0.7222199146, 0.8256138315; H, 1.0544124566, 1.9684107297, -1.1994807579; H, 3.2958554613, 0.9215118001, -1.3510729054; H, 3.779352637, -1.1804920071, -0.1042682199; H, 2.005357145, -2.2208534694, 1.3002159233; H, 0.227885634, -1.1749044273, 1.4597405652. Harmonic Frequencies (Number, Frequency ( $\mathrm{cm}^{-1}$ ), IR Intensity $\left.\left(\mathrm{km} \mathrm{mol}^{-1}\right)\right):$ 1, 52.1061, 0.9329; 2, 85.3802, 0.2983; 3, 115.5248, 3.1344; 4, 169.354, 3.4969; 5, 212.8516, 6.9117; 6, 333.7872, 2.0936; 7, 379.3319, 11.1384; 8, 418.6887, 0.4168; 9, 509.3918, 14.5894; 10, 523.6729, 10.0853; 11, 534.1862, 17.0448; 12, 559.6285, 49.7663; 13, 623.9316, 1.0586; 14, 658.9706, 25.9078; 15, 710.6586, 10.0608; 16, 720.5048, 112.8375; 17, 746.2767, 13.2875; 18, 791.1706, 18.1698; 19, 866.2188, 2.6289; 20, 878.8152, 71.4035; 21, 946.1843, 5.3922; 22, 981.787, 1.4774; 23, 1007.5441, 3.7029; 24, 1008.01, 2.0979; 25, 1031.4118, 124.9164; 26, 1055.8902, 50.6568; 27, 1109.6818, 3.6514; 28, 1120.354, 7.6533; 29, 1182.6036, 0.0162; 30, 1204.646, 2.1947; 31, 1216.3867, 130.3024; 32, 1331.634, 9.5231; 33, 1361.3076, 38.5195; 34, 1363.9655, 55.4537; 35, 1425.7795, 161.4685; 36, 1485.0993, 26.3559; 37, 1536.5518, 8.9643; 38, 1621.7036, 187.0520; 39, 1642.8556, 265.8852; 40, 1652.7965, 4.4717; 41, 2183.7751, 1482.5377; 42, 3121.2782, 62.9540; 43, 3144.357, 0.2933; 44, 3149.6039, 0.5115; 45, 3155.5496, 1.8724; 46, 3161.9765, 3.5285; 47, 3168.1666, 2.9346; 48, 3331.1557, 30.1693;

\section{Calculation summary for 1,3 Migration of Ph 1 sZ iE (acetonitrile)}

Geometry optimisation and frequency calculation: Program version = DEC-AXP-OSF/1-G03RevB.05. Method and basis set = RB3LYP/6-31G(d). Calculated Energy = -477.1141583 (hartree), $-1995.2914100106\left(\mathrm{~kJ} \mathrm{~mol}^{-1}\right)$. Higher level single-point calculation: Program = DEC-AXP-OSF/1-G03RevB.05. Method and basis set =

RB3LYP/6-311++G(3df,2p). Calculated Energy = -477.2834825 (hartree), -1995.999523815 (kJ mol ${ }^{-1}$ ). Structure definition (3D Coordinates: Atom, X coord/A, Y coord/Å, Z coord/Å): C, 0.8312919868, 0.3238120263, 1.728374; C, 0.8743449868, -0.1834040263, 3.044448; H, 1.7910569868, -0.5309100263, 1.256059; O, 0.9151119868, -0.0479920263, 4.203299; C, -0.4149290132, -0.2218380263, 0.938509; N, 1.6174820132, -0.3008110263, 1.39564; C, -0.2323240132, -0.0142090263, -0.532556; H, -1.6336450132, 0.4964550263, 2.407921; C, -1.2554220132, -0.4074800263, -1.412459; C, -1.1233100132, -0.2164770263, 2.786048; C, 0.0316449868, 0.3780259737, -3.305878; C, 1.0513099868, 0.7791669737, -2.440739; C, 
0.9223249868, 0.5813039737, -1.064101; H, -2.1488230132, -0.8704600263, -1.002734; H, -1.9211180132, 0.5361670263, -3.454847; H, 0.1349929868, 0.5263979737, -4.37979; H, 1.9501859868, 1.2508409737, -

2.834597; H, 1.7192369868, 0.9187059737, -0.405184. Harmonic Frequencies (Number, Frequency (cm ${ }^{-1}$ ), IR Intensity $\left(\mathrm{km} \mathrm{mol}^{-1}\right)$ ): 1, 34.7786, 0.1589; 2, 66.2449, 5.9294; 3, 110.1037, 2.3461; 4, 164.9816, 7.2600; 5, 234.2564, 5.1592; 6, 325.5856, 3.0408; 7, 408.4048, 11.1768; 8, 417.7847, 0.1653; 9, 491.0282, 20.4944; 10, 510.4547, 6.7417; 11, 533.9815, 5.5624; 12, 562.7866, 31.6322; 13, 626.2516, 0.3871; 14, 673.3636, 55.8085; 15, 692.5047, 4.7115; 16, 712.7497, 83.2735; 17, 797.2294, 14.8431; 18, 839.3267, 36.0714; 19, 860.0528, 74.3080; 20, 867.1745, 59.7268; 21, 947.5673, 9.4406; 22, 982.8373, 3.5897; 23, 990.5651, 88.9930; 24, 1007.5846, 3.0342; 25, 1008.9171, 0.0452; 26, 1053.7124, 0.2880; 27, 1108.8388, 5.9003; 28, 1147.0099, 5.4549; 29, 1180.1494, 9.8738; 30, 1194.3185, 114.0058; 31, 1209.4546, 21.1700; 32, 1322.6222, 57.0621; 33, 1351.7683, 29.7525; 34, 1362.3433, 3.3356; 35, 1434.5629, 400.1928; 36, 1487.1058, 57.9557; 37, 1537.9309, 11.6445; 38, 1622.1037, 86.8179; 39, 1646.0168, 89.3745; 40, 1654.2216, 18.6413; 41, 2181.9177, 1631.0528; 42, 3136.937, 50.4649; 43, 3145.3811, 0.5251; 44, 3154.4031, 4.8557; 45, 3163.4391, 10.1906; 46, 3173.673, 2.3130; 47, 3190.997, 1.2945; 48, 3293.9226, 16.2458;

\section{Calculation summary for 1,3 Migration of $\mathrm{Ph} 1 \mathrm{sZ}$ iZ (acetonitrile)}

Geometry optimisation and frequency calculation: Program version $=$ x86-Linux-G03RevB.01. Method and basis set = RB3LYP/6-31G(d). Calculated Energy = -477.1157227 (hartree), -1995.2979523314 (kJ mol $\left.{ }^{-1}\right)$. Higher level single-point calculation: Program = x86-Linux-G03RevB.01. Method and basis set $=$ RB3LYP/6-

311++G(3df,2p). Calculated Energy = -477.2855621 (hartree), -1996.0082207022 (kJ mol $\left.{ }^{-1}\right)$. Structure definition (3D Coordinates: Atom, X coord/Å, Y coord/Å, Z coord/Å): C, 0.845152395, -0.3214263023, 1.731072124; C, 0.8332515782, -0.2553709657, 3.0560361115; H, 1.7803338412, -0.627382141, 1.266849845; O, 0.833717998, -0.1918072371, 4.220732467; C, -0.3744086636, -0.0349301639, 0.9594880627; N, -1.4877070767, 0.1354192996, 1.590039344; C, -0.2144091593, 0.0297435224, 0.5267141821; H, -2.2423171494, 0.3826967674, 0.9372557536; C, -1.2482700608, -0.4293446091, 1.3595122408; C, -1.1282610083, -0.353961102, -2.7467396102; C, 0.0246103549, 0.187683687, 3.3227976985; C, 1.0591837684, 0.646372229, -2.5033955135; C, 0.9451671614, 0.5600666624, 1.1149706259; H, -2.1420675046, -0.8679247523, -0.9180601402; H, -1.9334284762, -0.7251617204, 3.3788571063; H, 0.1176425856, 0.247797005, -4.4060955867; H, 1.9584327929, 1.0732028576, 2.9447792495; H, 1.7535112682, 0.9302970368, -0.4872472227. Harmonic Frequencies (Number, Frequency

$\left(\mathrm{cm}^{-1}\right)$, IR Intensity $\left.\left(\mathrm{km} \mathrm{mol}^{-1}\right)\right): 1$, 45.0638, 0.3494; 2, 78.0543, 2.0738; 3, 100.0365, 2.0739; 4, 164.5019, 0.7254; 5, 225.9656, 2.3423; 6, 309.3077, 6.2865; 7, 397.7335, 4.2458; 8, 417.2984, 0.2562; 9, 485.5382, 14.6754; 10, 511.7421, 11.0207; 11, 541.7987, 4.9043; 12, 565.2826, 56.8688; 13, 624.3142, 0.5891; 14 , 673.3616, 38.5763; 15, 698.78, 21.0604; 16, 714.8621, 78.8559; 17, 783.2649, 54.6083; 18, 849.6972, 65.6148; 19, 860.497, 47.4013; 20, 864.037, 1.6682; 21, 945.2497, 7.4645; 22, 981.4352, 0.9598; 23, 1000.8723, 63.0700; 24, 1007.9175, 3.2947; 25, 1009.3369, 5.2160; 26, 1052.7872, 4.8486; 27, 1111.693, 1.7207; 28, 1143.5378, 3.8902; 29, 1182.6059, 0.0515; 30, 1207.2721, 12.4912; 31, 1213.423, 70.2610; 32, 1304.3185, 179.9108; 33, 1343.4501, 25.5614; 34, 1365.7594, 8.1922; 35, 1456.0912, 377.2096; 36, 1487.0229, 77.9556; 37, 1538.4386, 4.0402; 38, 1619.4416, 117.9266; 39, 1642.7946, 78.2608; 40, 1653.2265, 13.2732; 41, 2184.1758, 1643.1247; 42, 3144.6979, 0.3610; 43, 3150.6092, 0.4337; 44, 3156.7782, 19.1205; 45, 3158.4573, 34.2003; 46, 3165.1032, 8.4881; 47, 3170.294, 0.4785; 48, 3359.2267, 18.5116;

\section{Compound 1,3 Migration of $\mathrm{OCH}_{3}$}

\section{Calculation summary for 1,3 Migration of $\mathrm{OCH}_{3} 3 \mathrm{sE}$ (vacuum)}

Geometry optimisation and frequency calculation: Program version = x86-Linux-G03RevB.01. Method and 
basis set = RB3LYP/6-31G(d). Calculated Energy = -360.5923294 (hartree), -1507.9971215508 (kJ mol $\left.{ }^{-1}\right)$. Higher level single-point calculation: Program = x86-Linux-G03RevB.01. Method and basis set = RB3LYP/6-

$311+G(3 d f, 2 p)$. Calculated Energy = -360.733329 (hartree), -1508.586781878 $\left(\mathrm{kJ} \mathrm{mol}^{-1}\right)$. Structure definition (3D Coordinates: Atom, X coord/Å, Y coord/Å, Z coord/Å): C, 1.2420758103, -0.2236781572, -0.3662986635; C, 1.5857560208, -0.4108040487, 0.899654234; N, 1.9398665499, -0.4613484022, 2.0651150944; C, 0.1293585153, 0.0960291264, -0.7887342034; O, -0.985031765, 0.162651027, 0.2635753172; O, 0.4625908571, 0.2775070261, -1.9421179629; H, 1.9884283232, -0.3209356599, -1.1462205689; H, 1.9076760264, -1.3631261303, 2.5445226696; C, -2.3410235471, 0.4870760862, -0.0757702938; H, 2.8820386158, 0.5031435141, 0.871027789; H, -2.3939283235, 1.4641031345, -0.5637266997; H, 2.7629208948, -0.2667485079, -0.7461741247. Harmonic Frequencies (Number, Frequency $\left(\mathrm{cm}^{-1}\right), \mathrm{IR}^{-}$ Intensity ( $\left.\mathrm{km} \mathrm{mol}^{-1}\right)$ ): 1, 88.7455, 1.1922; 2, 124.3501, 0.3323; 3, 134.9798, 0.4957; 4, 174.5801, 9.8301; 5, 295.6384, 10.9153; 6, 409.0203, 11.9161; 7, 465.8385, 44.7501; 8, 520.4316, 33.8728; 9, 532.3424, 0.6270; 10, 729.1778, 4.5972; 11, 771.5408, 43.2351; 12, 853.6367, 41.9398; 13, 865.12, 25.2389; 14, 968.456, 394.6632; 15, 1079.2712, 42.8468; 16, 1139.7876, 59.0815; 17, 1185.5554, 0.9175; 18, 1219.3225, 8.1678; 19, 1287.8792, 423.4730; 20, 1406.3306, 49.3747; 21, 1494.1287, 20.2217; 22, 1512.7572, 5.4418; 23, 1527.0189, 6.5189; 24, 1807.375, 398.3800; 25, 2143.8117, 516.9869; 26, 3073.7572, 33.2811; 27, 3147.5766, 23.3983; 28, 3179.0126, 16.6719; 29, 3234.8909, 5.1669; 30, 3479.7331, 24.7523;

\section{Calculation summary for 1,3 Migration of $\mathrm{OCH}_{3} 3 \mathrm{sZ}$ (vacuum)}

Geometry optimisation and frequency calculation: Program version $=$ x86-Linux-G03RevB.01. Method and basis set = RB3LYP/6-31G(d). Calculated Energy = -360.5919236 (hartree), -1507.9954244952 (kJ mol-1). Higher level single-point calculation: Program = x86-Linux-G03RevB.01. Method and basis set = RB3LYP/6-

311+G(3df,2p). Calculated Energy = -360.7331062 (hartree), $-1508.5858501284\left(\mathrm{~kJ} \mathrm{~mol}^{-1}\right)$. Structure definition (3D Coordinates: Atom, X coord $/ \AA$, Y coord $/ \AA$, Z coord/Å): C, 0.8021238483, -0.7943143703, 0.0137336955; C, 2.0157876916, -0.2612942738, 0.0057922244; H, 0.6884395629, -1.8720748163, 0.045622975; N, 3.1284947346, 0.2145404066, -0.1286308379; C, -0.3869508947, 0.070721407, 0.0054335361; O, -0.3908792545, 1.2862235456, 0.0037562041; O, -1.5127244506, -0.6874726869, 0.0038971168; H, 3.6516640908, 0.4800304026, 0.7079187474; C, -2.7405785824, 0.0547681098, 0.0062769535; H, -3.533812944, -0.6937504846, -0.0068761198; H, -2.8144658653, 0.6918336659, 0.8794316923; H, -2.8047507226, 0.6828862803, -0.8990030113. Harmonic Frequencies (Number, Frequency $\left(\mathrm{cm}^{-1}\right)$, IR Intensity $\left.\left(\mathrm{km} \mathrm{mol}^{-1}\right)\right): 1,76.7923,3.9531 ; 2,123.7288,0.5233 ; 3,128.2529,0.9768 ; 4,184.9906$, 7.5430; 5, 290.5216, 14.9351; 6, 402.1396, 6.7735; 7, 467.013, 45.9713; 8, 496.8352, 0.2686; 9, 528.7172, 37.2094; 10, 743.5601, 9.2405; 11, 772.8453, 43.3945; 12, 858.0607, 57.3965; 13, 949.1211, 10.2107; 14, 963.0383, 431.7643; 15, 1033.8936, 9.2965; 16, 1145.354, 1.1328; 17, 1185.5514, 0.8980; 18, 1212.3163, 182.4101; 19, 1228.4014, 379.2508; 20, 1442.4783, 162.6254; 21, 1496.6441, 49.6269; 22, 1511.5281, 5.5493; 23, 1526.0878, 6.5599; 24, 1794.9935, 212.8023; 25, 2150.1947, 555.4889; 26, 3073.0359, 34.4760; 27, 3146.3591, 23.4485; 28, 3178.4273, 20.0235; 29, 3228.3488, 4.7098; 30, 3481.9263, 26.8111;

\section{Calculation summary for 1,3 Migration of $\mathrm{OCH}_{3}$ TS2 iE (vacuum)}

Geometry optimisation and frequency calculation: Program version = x86-Linux-G03RevB.01. Method and basis set = RB3LYP/6-31G(d). Calculated Energy = -360.5364766 (hartree), -1507.7635451412 (kJ mol $\left.{ }^{-1}\right)$. Higher level single-point calculation: Program = x86-Linux-G03RevB.01. Method and basis set = RB3LYP/6311+G(3df,2p). Calculated Energy = -360.6771358 (hartree), $-1508.3517819156\left(\mathrm{~kJ} \mathrm{~mol}^{-1}\right)$. Structure definition (3D Coordinates: Atom, X coord/A, Y coord/A, Z coord/Å): C, 0.9761161168, 0.2804012101, 1.0050786108; C, 1.0429646869, 0.2653916554, 0.395629968; O, 1.7744681874, 0.2450899247, 1.338777929; 
C, -0.3849908727, 0.1211065216, -1.2272319821; N, -1.4607675291, -0.0448944965, -1.7938917086; O, 0.4998399202, 0.2660957875, 0.5680805752; H, 1.7599127423, 0.4156729675, -1.7347239263; C, 1.0675982055, -0.788732654, 1.3582698925; H, -1.4881524737, -0.3462350092, -2.7616430979; H, 2.1520724496, -0.7044104492, 1.2710346119; H, -0.7338241134, -1.7703723483, 1.0032537969; H, 0.7564674925, -0.6388797808, 2.394916937. Harmonic Frequencies (Number, Frequency ( $\mathrm{cm}^{-1}$ ), IR Intensity $\left(\mathrm{km} \mathrm{mol}^{-1}\right)$ ): 1, -436.1702, 101.5169; 2, 59.5428, 0.9362; 3, 138.2858, 5.4929; 4, 182.989, 12.5315; 5, 226.7251, 2.5926; 6, 326.8683, 20.7829; 7, 424.285, 147.1890; 8, 552.705, 3.9942; 9, 583.5746, 13.0070; 10, 631.0263, 26.0684; 11, 651.0457, 75.7823; 12, 692.0691, 58.3110; 13, 723.1513, 17.4571; 14, 848.0626, 182.7914; 15, 1031.9801, 169.4460; 16, 1042.2507, 62.6112; 17, 1186.1936, 0.6612; 18, 1196.6697, 10.3871; 19, 1221.4035, 31.6719; 20, 1318.957, 15.1670; 21, 1471.6002, 0.1812; 22, 1515.738, 11.0454; 23, 1517.9852, 5.1902; 24, 1931.3956, 1478.4395; 25, 2017.1614, 23.1670; 26, 3059.5729, 30.7611; 27, 3145.9131, 16.1800; 28, 3177.4685, 6.5317; 29, 3292.9005, 1.8333; 30, 3598.204, 96.9381;

\section{Calculation summary for 1,3 Migration of $\mathrm{OCH}_{3}$ TS2 iZ (vacuum)}

Geometry optimisation and frequency calculation: Program version = x86-Linux-G03RevB.01. Method and basis set = RB3LYP/6-31G(d). Calculated Energy = -360.5401076 (hartree), -1507.7787299832 (kJ mol $\left.{ }^{-1}\right)$. Higher level single-point calculation: Program = x86-Linux-G03RevB.01. Method and basis set = RB3LYP/6311+G(3df,2p). Calculated Energy = -360.6800898 (hartree), $-1508.3641355436\left(\mathrm{~kJ} \mathrm{~mol}^{-1}\right)$. Structure definition (3D Coordinates: Atom, X coord/A, Y coord/A, Z coord/Å): C, 1.1724323314, 0.4233460362, 0.6842118202; C, 1.0368731045, 0.2056366479, 0.6851078932; N, 1.5536796411, 0.0229569629, 1.7864933227; C, -0.1393042328, 0.3173406832, -1.1322147176; O, -0.7043650479, 0.1660285008, 0.3967012965; O, -0.8948707566, 0.290373931, -2.0486078445; H, 2.0675962819, 0.6550762666, 1.2405110101; C, -1.4441251765, -1.0343618803, 0.6555954626; H, 1.0182796712, 0.0390299985, 2.64467702; H, -1.7980286974, -1.0015081649, 1.6893155562; H, -2.2997597577, -1.0510447568, 0.0250772075; H, -0.8252147103, -1.9252404594, 0.4957338582. Harmonic Frequencies (Number, Frequency $\left(\mathrm{cm}^{-1}\right)$, IR Intensity $\left.\left(\mathrm{km} \mathrm{mol}^{-1}\right)\right): 1,-417.2071,140.2305 ; 2,97.1156,0.5416 ; 3,139.7036,2.2136 ; 4$, 190.2443, 6.9117; 5, 220.5339, 2.1225; 6, 302.3671, 13.7758; 7, 452.1763, 196.0162; 8, 525.8283, 20.2757; 9, 596.5235, 2.9023; 10, 621.4816, 8.9380; 11, 653.6273, 118.0696; 12, 672.5593, 26.8484; 13, 703.3279, 15.4214; 14, 876.5656, 316.1077; 15, 1015.3415, 170.6239; 16, 1045.4724, 24.2746; 17, 1188.2569, 0.6934; 18, 1189.2464, 6.1612; 19, 1216.1137, 31.6614; 20, 1333.1747, 13.4026; 21, 1476.7688, 0.9596; 22, 1518.7977, 4.3788; 23, 1519.8257, 10.2523; 24, 1949.1975, 1371.5904; 25, 2040.6161, 74.2430; 26, 3050.9503, 34.8688; 27, 3133.9661, 20.7295; 28, 3155.3335, 9.3481; 29, 3296.8479, 3.0422; 30, 3630.3525, 92.7824;

\section{Calculation summary for 1,3 Migration of $\mathrm{OCH}_{3} 1 \mathrm{sE} \mathrm{iE} \mathrm{(vacuum)}$}

Geometry optimisation and frequency calculation: Program version = x86-Linux-G03RevB.01. Method and basis set = RB3LYP/6-31G(d). Calculated Energy = -360.585942 (hartree), -1507.970409444 $\left(\mathrm{kJ} \mathrm{mol}^{-1}\right)$. Higher level single-point calculation: Program = x86-Linux-G03RevB.01. Method and basis set = RB3LYP/6-

311+G(3df,2p). Calculated Energy = -360.7276094 (hartree), $-1508.5628625108\left(\mathrm{~kJ} \mathrm{~mol}^{-1}\right)$. Structure definition (3D Coordinates: Atom, X coord/Å, Y coord/A, Z coord/Å): C, 1.2979271717, 0.2526518604, 0.0912407874; C, 1.191795537, 0.1790568027, 1.4118787624; O, 1.1145953771, 0.1175458724, 2.5728390819; C, 0.1306287925, 0.0569195076, -0.7807119945; N, 0.0834095402, 0.0982875213, 2.0542625809; O, -0.9792795905, -0.1916740429, -0.0406376633; H, 2.2750471747, 0.4622613569, 0.32731559; C, -2.1939537739, -0.4025497827, -0.7695743905; H, 1.0010124557, 0.2952736327, 2.4528106668; H, -2.4440296259, 0.4784500932, -1.3671606259; H, -2.9569482079, -0.5835245032, 0.0108297591; H, -2.0998612342, -1.2639181923, -1.4366556295. Harmonic Frequencies (Number, Frequency 
$\left(\mathrm{cm}^{-1}\right)$, IR Intensity $\left.\left(\mathrm{km} \mathrm{mol}^{-1}\right)\right): 1,84.0025,0.0795 ; 2,120.4492,0.4343 ; 3,160.9928,10.1853 ; 4,177.2506$, 7.5078; 5, 313.6805, 14.0468; 6, 411.8058, 2.0853; 7, 507.7163, 5.1760; 8, 530.4934, 4.7781; 9, 541.9712, 17.9650; 10, 707.609, 36.4095; 11, 723.1539, 1.4014; 12, 819.5269, 76.4469; 13, 872.4829, 17.2114; 14, 1064.4216, 41.0983; 15, 1119.4801, 150.4561; 16, 1144.9037, 11.4196; 17, 1185.2761, 0.7154; 18, 1225.4343, 22.2100; 19, 1372.9497, 211.0247; 20, 1399.4229, 151.2888; 21, 1496.525, 49.1084; 22, 1506.2036, 5.3049; 23, 1527.4268, 9.3939; 24, 1719.396, 289.4403; 25, 2235.0331, 771.4902; 26, 3071.4135, 33.0740; 27, 3145.376, 23.4708; 28, 3173.5307, 19.0456; 29, 3236.7794, 7.8375; 30, 3505.5554, 1.9787;

\section{Calculation summary for $\mathbf{1 , 3}$ Migration of $\mathrm{OCH}_{3} 1 \mathrm{sE}$ iZ (vacuum)}

Geometry optimisation and frequency calculation: Program version = x86-Linux-G03RevB.01. Method and basis set = RB3LYP/6-31G(d). Calculated Energy = -360.582251 (hartree), -1507.954973682 (kJ mol $\left.{ }^{-1}\right)$. Higher level single-point calculation: Program = x86-Linux-G03RevB.01. Method and basis set = RB3LYP/6$311+G(3 d f, 2 p)$. Calculated Energy = -360.7239323 (hartree), $-1508.5474848786\left(\mathrm{~kJ} \mathrm{~mol}^{-1}\right)$. Structure definition (3D Coordinates: Atom, X coord/A, Y coord/Å, Z coord/Å): C, 1.2840864553, -0.3249646411, 0.0756653813; C, 0.0561267211, -0.0043420252, -0.8026754751; N, 0.0352499352, 0.016652562, 2.0761052356; C, 1.3358403326, -0.3544858432, 1.2484570473; O, -0.9667372641, 0.244402151, 0.0801575744; O, 1.410823749, -0.3879182343, 2.4118435608; H, 2.1844944799, -0.5465488031, 0.6350914519; C, -2.2393483574, 0.5741581355, -0.4641363764; H, -0.8818157178, 0.2541001828, 2.4545402791; H, -2.8966505994, 0.7305600493, 0.392083808; H, -2.1923716932, 1.493996902, 1.060182175; H, -2.633328805, -0.2427413555, -1.081421221. Harmonic Frequencies (Number, Frequency

$\left(\mathrm{cm}^{-1}\right)$, IR Intensity $\left.\left(\mathrm{km} \mathrm{mol}^{-1}\right)\right): 1$, 88.1221, 0.0958; 2, 118.8109, 1.7937; 3, 146.5583, 4.0671; 4, 208.8859, 0.2830; 5, 316.4769, 8.7428; 6, 416.7365, 5.8474; 7, 525.6841, 10.5920; 8, 531.25, 9.4491; 9, 536.1682, 44.0604; 10, 712.4015, 13.6048; 11, 713.334, 16.9833; 12, 806.9941, 77.9352; 13, 837.0865, 22.8421; 14, 1064.1893, 52.9375; 15, 1124.252, 81.6983; 16, 1142.0901, 106.1187; 17, 1182.3255, 0.9370; 18, 1223.1347, 29.7540; 19, 1332.2355, 171.3366; 20, 1414.877, 96.3528; 21, 1494.8125, 22.4154; 22, 1520.8981, 6.2069; 23 , 1534.7252, 7.8188; 24, 1726.5108, 310.3242; 25, 2235.8318, 772.4752; 26, 3037.752, 32.1870; 27, 3100.7752, 36.0848; 28, 3170.2221, 13.0593; 29, 3247.8848, 17.4566; 30, 3504.0412, 2.1818;

\section{Calculation summary for $\mathbf{1 , 3}$ Migration of $\mathrm{OCH}_{3} 1 \mathrm{sZ}$ iE (vacuum)}

Geometry optimisation and frequency calculation: Program version = x86-Linux-G03RevB.01. Method and basis set = RB3LYP/6-31G(d). Calculated Energy = -360.582181 (hartree), -1507.954680942 (kJ mol $\left.{ }^{-1}\right)$. Higher level single-point calculation: Program = x86-Linux-G03RevB.01. Method and basis set = RB3LYP/6$311+G(3 d f, 2 p)$. Calculated Energy = -360.7241987 (hartree), $-1508.5485989634\left(\mathrm{~kJ} \mathrm{~mol}^{-1}\right)$. Structure definition (3D Coordinates: Atom, X coord/A, Y coord/A, Z coord/Å): C, 1.0512419612, 0.0043568366, 0.36188858; C, 1.2100394256, -0.1217505518, 1.6688112226; H, 1.9460521915, 0.100541419, 0.2435444913; O, 1.3445565687, -0.2333233571, 2.8228231934; C, -0.2731054266, 0.0188882477, 0.2918705216; N, -1.4452007384, -0.0771390212, 0.2048749052; O, -0.0894066088, 0.1611670628, 1.6262411292; C, -1.2795216368, 0.1952757379, -2.4216697363; H, -1.422880524, -0.1790463627, 1.2198662794; H, -0.9342852452, 0.3128844832, -3.450200629; H, -1.8483600978, -0.7317675577, 2.3073222549; H, -1.9172467758, 1.0339898988, -2.1285370225. Harmonic Frequencies (Number, Frequency $\left(\mathrm{cm}^{-1}\right)$, IR Intensity $\left.\left(\mathrm{km} \mathrm{mol}^{-1}\right)\right): 1,46.5768,1.1162 ; 2,124.1529,1.4060 ; 3,156.4406,15.0535 ; 4,188.7613$, 4.1448; 5, 305.2331, 13.1759; 6, 411.033, 7.2479; 7, 517.94, 4.0535; 8, 519.0411, 0.1344; 9, 535.7658, 23.6622; 10, 711.179, 49.1100; 11, 723.9838, 5.9879; 12, 807.5113, 49.3166; 13, 934.3386, 25.0425; 14, 1003.64, 37.0846; 15, 1118.7171, 136.7470; 16, 1138.2865, 67.2299; 17, 1186.042, 0.6286; 18, 1225.2465, 22.6037; 19, 1317.7514, 183.9081; 20, 1445.7964, 190.5609; 21, 1499.4139, 78.1555; 22, 1506.071, 5.3153; 23, 1526.4118, 9.3102; 24, 1704.8714, 175.6212; 25, 2230.7868, 730.6304; 26, 3070.4815, 33.8465; 27, 
3143.7512, 23.3561; 28, 3172.0002, 22.6389; 29, 3224.5586, 15.9790; 30, 3492.2926, 2.7999;

\section{Calculation summary for 1,3 Migration of $\mathrm{OCH}_{3} 1 \mathrm{sZ}$ iZ (vacuum)}

Geometry optimisation and frequency calculation: Program version = x86-Linux-G03RevB.01. Method and basis set = RB3LYP/6-31G(d). Calculated Energy = -360.5763666 (hartree), -1507.9303651212 (kJ mol $\left.{ }^{-1}\right)$. Higher level single-point calculation: Program = x86-Linux-G03RevB.01. Method and basis set = RB3LYP/6311+G(3df,2p). Calculated Energy = -360.7178 (hartree), -1508.5218396 $\left(\mathrm{kJ} \mathrm{mol}^{-1}\right)$. Structure definition (3D Coordinates: Atom, X coord/Å, Y coord/Å, Z coord/Å): C, 0.6636220303, 0.098775024, 0.4309720868; C, 0.6395825395, -0.154009363, 1.7349569268; H, 1.6042498061, 0.4330481021, 0.0140528987; O, 0.6564040781, -0.3653604194, 2.8785058847; C, -0.5676718749, -0.0885797275, -0.3488111729; N, 1.6387793413, -0.4869928643, 0.2176127803; O, -0.5325499218, 0.1748079691, -1.6906411538; C, 0.6632853384, 0.619732296, -2.3233816248; H, -2.3944135876, -0.5628890596, -0.4668718075; H, 0.4084977053, 0.7499027734, -3.3767066027; H, 1.0030997435, 1.5807878422, -1.9182495986; H, 1.4662802716, -0.1229893828, -2.2408494942. Harmonic Frequencies (Number, Frequency $\left(\mathrm{cm}^{-1}\right), \mathrm{IR}^{-1}$ Intensity ( $\left.\mathrm{km} \mathrm{mol}^{-1}\right)$ ): 1, 46.244, 0.1790; 2, 95.6694, 0.6451; 3, 140.5642, 0.8189; 4, 228.2177, 0.0190; 5, 280.51, 0.5126; 6, 441.3377, 17.0317; 7, 494.67, 13.3067; 8, 528.7199, 6.3253; 9, 549.1366, 30.7571; 10, 614.9087, 1.8947; 11, 685.9707, 8.0749; 12, 841.1457, 109.5947; 13, 917.4688, 12.4175; 14, 1034.0442, 98.9133; 15, 1156.4871, 86.3867; 16, 1163.2797, 31.4543; 17, 1177.0071, 0.3981; 18, 1222.4643, 12.3520; 19, 1290.3795, 228.2130; 20, 1459.1587, 205.2797; 21, 1510.3534, 72.0659; 22, 1526.4722, 5.4850; 23, 1534.4123, 10.5126; 24, 1712.1728, 225.5570; 25, 2241.8519, 750.4887; 26, 3036.4658, 41.3936; 27 , 3102.0264, 38.2840; 28, 3159.9848, 20.4296; 29, 3251.7333, 8.6538; 30, 3480.9127, 3.4743;

\section{Calculation summary for 1,3 Migration of $\mathrm{OCH}_{3} 3 \mathrm{sE}$ (acetonitrile)}

Geometry optimisation and frequency calculation: Program version = x86-Linux-G03RevB.01. Method and basis set = RB3LYP/6-31G(d). Calculated Energy = -360.6075735 (hartree), -1508.060872377 (kJ mol $\left.{ }^{-1}\right)$. Higher level single-point calculation: Program = x86-Linux-G03RevB.01. Method and basis set = RB3LYP/6311++G(3df,2p). Calculated Energy = -360.7494132 (hartree), -1508.6540460024 (kJ mol $\left.{ }^{-1}\right)$. Structure definition (3D Coordinates: Atom, X coord/Å, Y coord/Å, Z coord/Å): C, 1.2418427926, -0.2179560705, 0.3666700931; C, 1.5845942271, -0.4125314387, 0.9030701642; N, 1.9386483608, -0.4697069585, 2.0634659945; C, -0.1240798685, 0.0955278472, -0.7837296802; O, -0.9834611785, 0.177375082, 0.2604165931; O, -0.4632115182, 0.2681870278, -1.9447739924; H, 1.9977239465, -0.3152304634, 1.1430212809; H, 1.9236791939, -1.3822849759, 2.5574511174; C, -2.3461562142, 0.4891410096, 0.0773607869; H, -2.8826617733, 0.5112401433, 0.8717305561; H, -2.4075829288, 1.4620094364, 0.5726824904; H, -2.7655210119, -0.2773703945, -0.7347382931. Harmonic Frequencies (Number, Frequency $\left(\mathrm{cm}^{-1}\right)$, IR Intensity $\left.\left(\mathrm{km} \mathrm{mol}^{-1}\right)\right): 1,72.6054,1.3238 ; 2,122.4692,0.3228 ; 3,140.9983,1.4109 ; 4,170.4895$, 13.1384; 5, 295.0926, 22.4750; 6, 412.0515, 22.8538; 7, 466.5289, 75.7273; 8, 518.3266, 60.4697; 9, 535.1301, 4.1530; 10, 725.0539, 11.0733; 11, 767.1128, 72.9540; 12, 825.712, 93.9924; 13, 859.6743, 30.9716; 14, 893.9459, 755.1097; 15, 1071.3463, 75.8048; 16, 1136.4607, 74.8954; 17, 1180.9362, 1.6999; 18, 1215.3705, 16.6841; 19, 1277.6342, 589.5454; 20, 1406.1075, 76.6734; 21, 1491.6318, 43.0929; 22, 1507.0293, 7.7594; 23, 1518.3691, 7.2716; 24, 1745.5876, 935.8056; 25, 2129.6733, 947.7864; 26, 3070.9764, 34.6417; 27, 3146.7054, 26.3147; 28, 3158.43, 86.5195; 29, 3172.0835, 370.3526; 30, 3179.283, 17.6092;

\section{Calculation summary for 1,3 Migration of $\mathrm{OCH}_{3} 3 \mathrm{sZ}$ (acetonitrile)}

Geometry optimisation and frequency calculation: Program version = x86-Linux-G03RevB.01. Method and 
basis set = RB3LYP/6-31G(d). Calculated Energy = -360.6074296 (hartree), -1508.0602705872 (kJ mol $\left.{ }^{-1}\right)$. Higher level single-point calculation: Program = x86-Linux-G03RevB.01. Method and basis set = RB3LYP/6-

311++G(3df,2p). Calculated Energy = -360.7493925 (hartree), -1508.653959435 (kJ mol $\left.{ }^{-1}\right)$. Structure definition (3D Coordinates: Atom, X coord/Å, Y coord/Å, Z coord/Å): C, 0.8004785059, -0.7955227978, 0.0031449394; C, 2.0171504492, -0.2592903085, 0.009362073; H, 0.6932063343, -1.8780581007, 0.0269663429; N, 3.1265951616, 0.2183241348, -0.110393458; C, -0.3827998321, 0.0640920215, 0.0035914603; O, -0.3890891095, 1.2867736758, -0.0227901065; O, -1.5120840669, -0.6835663554, 0.013207806; H, 3.6699098562, 0.4690115545, 0.7378662379; C, -2.7460914969, 0.0548521206, 0.0041145898; H, -3.5353539753, -0.6974989781, 0.0184095824; H, -2.8162966017, 0.6985119015, 0.8852535764; H, -2.8206720896, 0.6693199017, -0.8972639807. Harmonic Frequencies (Number, Frequency $\left(\mathrm{cm}^{-1}\right)$, IR Intensity $\left.\left(\mathrm{km} \mathrm{mol}^{-1}\right)\right): 1$, 43.3999, 6.3489; 2, 126.8532, 2.0970; 3, 130.777, 1.9366; 4, 179.9644, 10.0747; 5, 291.6971, 30.4875; 6, 405.8984, 6.8297; 7, 467.4744, 77.1807; 8, 499.9271, 1.5148; 9, 527.2971, 65.6918; 10, 741.0374, 19.0913; 11, 767.911, 74.4068; 12, 831.4017, 89.5524; 13, 888.3642, 821.5463; 14, 947.6438, 4.2382; 15, 1026.6977, 17.2630; 16, 1142.5938, 3.0411; 17, 1181.1998, 1.5936; 18, 1206.4463, 486.7421; 19, 1220.3803, 385.4566; 20, 1437.5657, 265.3701; 21, 1493.7921, 95.4163; 22, 1505.9687, 7.8918; 23, 1517.4853, 5.8836; 24, 1737.1185, 411.9885; 25, 2133.5117, 992.0836; 26, 3070.5521, 34.5602; 27 , 3146.1411, 25.8566; 28, 3154.9371, 95.9263; 29, 3168.7781, 363.7314; 30, 3179.068, 16.6940;

\section{Calculation summary for 1,3 Migration of $\mathrm{OCH}_{3}$ TS2 iE (acetonitrile)}

Geometry optimisation and frequency calculation: Program version = x86-Linux-G03RevB.01. Method and basis set = RB3LYP/6-31G(d). Calculated Energy = -360.5508942 (hartree), $-1507.8238395444\left(\mathrm{~kJ} \mathrm{~mol}^{-1}\right)$. Higher level single-point calculation: Program = x86-Linux-G03RevB.01. Method and basis set = RB3LYP/6311++G(3df,2p). Calculated Energy = -360.6920305 (hartree), -1508.414071551 (kJ mol $\left.{ }^{-1}\right)$. Structure definition (3D Coordinates: Atom, X coord/Å, Y coord/A, Z coord/Å): C, 0.9766522783, 0.2488951043, 1.0080188544; C, 1.0449399959, 0.2718260523, 0.3830446149; O, 1.7815625116, 0.2896821789, 1.3328373447; C, -0.3982524252, 0.1056500076, -1.2079071384; N, -1.4752837471, -0.0502705783, 1.771324029; O, -0.4962805308, 0.2711397433, 0.567376491; H, 1.7564808807, 0.3380639958, 1.7542322139; C, -1.0596925455, -0.8007236239, 1.3496407926; H, -1.477732664, -0.2740017284, 2.7779596376; H, -2.1422497264, -0.7519082368, 1.2208251681; H, -0.6783602075, -1.7705614263, 1.0124519899; H, -0.7952917203, -0.6301591756, 2.3959157227. Harmonic Frequencies (Number, Frequency $\left(\mathrm{cm}^{-1}\right)$, IR Intensity $\left.\left(\mathrm{km} \mathrm{mol}^{-1}\right)\right): 1,-408.1178,295.7161 ; 2,79.1818,1.5431 ; 3,135.9719,7.7022 ; 4$, 186.6843, 24.2659; 5, 233.2005, 8.3978; 6, 319.3353, 16.8578; 7, 436.1543, 184.8667; 8, 550.8999, 4.1197; 9, 584.5165, 31.6085; 10, 627.748, 30.5141; 11, 649.7253, 142.7955; 12, 683.7794, 85.2411; 13, 720.7925, 16.9013; 14, 839.1633, 288.3192; 15, 1007.0681, 315.0777; 16, 1030.5831, 19.3020; 17, 1182.6023, 1.1945; 18, 1193.9048, 11.6890; 19, 1216.7127, 47.6581; 20, 1311.6701, 24.9847; 21, 1469.0723, 1.1660; 22, 1507.34, 17.7999; 23, 1509.0604, 5.3545; 24, 1849.5502, 2865.6688; 25, 1988.8846, 91.0387; 26, 3061.4628, 27.5488; 27, 3149.8549, 13.7290; 28, 3176.4033, 5.8448; 29, 3226.2969, 30.9022; 30, 3266.7195, 549.7461;

\section{Calculation summary for 1,3 Migration of $\mathrm{OCH}_{3}$ TS2 iZ (acetonitrile)}

Geometry optimisation and frequency calculation: Program version = x86-Linux-G03RevB.01. Method and basis set = RB3LYP/6-31G(d). Calculated Energy = -360.5536469 (hartree), -1507.8353513358 (kJ mol $\left.{ }^{-1}\right)$. Higher level single-point calculation: Program = x86-Linux-G03RevB.01. Method and basis set = RB3LYP/6311++G(3df,2p). Calculated Energy = -360.6941863 (hartree), -1508.4230871066 (kJ mol $\left.{ }^{-1}\right)$. Structure definition (3D Coordinates: Atom, X coord/Å, Y coord/A, Z coord/Å): C, 1.1815527956, 0.4026588408, 0.682912503; C, 1.0056512699, 0.2087769827, 0.6938574356; N, 1.5127869128, 0.0700530494, 
1.8061534801; C, -0.1230545707, 0.3056624301, -1.1357204824; O, -0.6980105125, 0.16544439903, 0.3778723377; O, -0.8764918047, 0.2892475862, -2.064111101; H, 2.0929210219, 0.6075236846, 1.230879541; C, -1.4287265178, -1.045080693, 0.6602169979; H, 0.95587904, 0.0284026374, 2.667259194; H, -1.6575961292, -1.0531423056, 1.7282986482; H, -2.3554735898, -1.0137315368, 0.0818717572; H, 0.841758058, -1.929061801, 0.3876369983. Harmonic Frequencies (Number, Frequency $\left(\mathrm{cm}^{-1}\right)$, IR Intensity $\left.\left(\mathrm{km} \mathrm{mol}^{-1}\right)\right):$ 1, -400.0559, 328.8309; 2, 109.5255, 1.5600; 3, 135.825, 4.0174; 4, 201.1333, 18.3972; 5, 225.5105, 7.2650; 6, 307.0351, 17.3159; 7, 471.3326, 285.3410; 8, 538.0741, 25.6858; 9, 596.0175, 17.5134; 10, 623.9207, 19.5587; 11, 647.2669, 180.5178; 12, 668.3215, 46.3941; 13, 706.5474, 21.7611; 14, 890.6072, 573.1903; 15, 991.9121, 237.2302; 16, 1034.4361, 40.5655; 17, 1185.2121, 1.6094; 18, 1188.0899, 5.8771; 19 , 1215.1252, 33.5984; 20, 1322.0369, 19.8122; 21, 1472.4203, 0.6402; 22, 1508.4042, 12.0891; 23, 1509.8004, 9.6119; 24, 1873.987, 2738.4300; 25, 2003.0539, 34.3027; 26, 3056.645, 27.8133; 27, 3144.5875, 14.3170; 28, 3164.9198, 6.0913; 29, 3226.1613, 34.4864; 30, 3333.8036, 460.9796;

\section{Calculation summary for $\mathbf{1 , 3}$ Migration of $\mathrm{OCH}_{3} 1 \mathrm{sE} i E$ (acetonitrile)}

Geometry optimisation and frequency calculation: Program version = x86-Linux-G03RevB.01. Method and basis set $=$ RB3LYP/6-31G(d). Calculated Energy = -360.5962394 (hartree), $-1508.0134731708\left(\mathrm{~kJ} \mathrm{~mol}^{-1}\right)$. Higher level single-point calculation: Program = x86-Linux-G03RevB.01. Method and basis set $=$ RB3LYP/6$311++G(3 d f, 2 p)$. Calculated Energy $=-360.7384109$ (hartree), $-1508.6080343838\left(\mathrm{~kJ} \mathrm{~mol}^{-1}\right)$. Structure definition (3D Coordinates: Atom, X coord/Å, Y coord/Å, Z coord/Å): C, 1.2966091552, 0.2526424513, 0.0897002571; C, 1.1953473326, 0.1802106559, 1.4088741428; O, 1.11619586, 0.1183071982, 2.5716355692; C, 0.1298308082, 0.0564630905, -0.7795416522; N, 0.0933196871, 0.0999647232, -2.0550621506; O, 0.9841438645, -0.1931941704, -0.041459952; H, 2.2778114983, 0.4632367636, -0.3327419547; C, 2.2020277292, -0.4041582818, -0.7666261429; H, 1.0271688502, 0.3001801998, -2.4364884301; H, 2.4565338299, 0.4776166082, -1.3619162946; H, -2.9642305435, -0.5850396079, -0.0069852893; H, 2.1124271497, -1.2675987433, -1.4322775426. Harmonic Frequencies (Number, Frequency $\left(\mathrm{cm}^{-1}\right)$, IR Intensity (km mol$\left.{ }^{-1}\right)$ ): 1, 80.2567, 0.1512; 2, 118.6489, 1.1711; 3, 155.0836, 23.9397; 4, 177.3648, 4.7359; 5, 303.8783, 19.1091; 6, 410.9481, 0.7870; 7, 518.0152, 3.9642; 8, 532.1631, 6.5204; 9, 537.4325, 31.3093; 10, 707.6172, 57.1141; 11, 719.7356, 1.3865; 12, 809.1271, 121.1080; 13, 863.6525, 40.3678; 14, 1067.4945, 40.2449; 15, 1114.4897, 232.5371; 16, 1133.1385, 50.9473; 17, 1181.7959, 1.4234; 18, 1219.6476, 33.4050; 19, 1362.2207, 396.7783; 20, 1394.544, 172.2691; 21, 1492.4976, 77.8571; 22, 1500.6613, 7.2514; 23, 1520.3767, 13.6039; 24, 1691.6369, 623.3357; 25, 2192.8818, 1444.5242; 26, 3065.2024, 36.6985; 27, 3138.4919, 31.2123; 28, 3145.4447, 65.3325; 29, 3170.278, 20.8336; 30, 3328.1811, 68.3674;

\section{Calculation summary for $\mathbf{1 , 3}$ Migration of $\mathrm{OCH}_{3} 1 \mathrm{sE} \mathrm{iZ} \mathrm{(acetonitrile)}$}

Geometry optimisation and frequency calculation: Program version $=$ x86-Linux-G03RevB.01. Method and basis set $=$ RB3LYP/6-31G(d). Calculated Energy = -360.5922705 (hartree), $-1507.996875231\left(\mathrm{~kJ} \mathrm{~mol}^{-1}\right)$. Higher level single-point calculation: Program = x86-Linux-G03RevB.01. Method and basis set $=$ RB3LYP/6$311++G(3 d f, 2 p)$. Calculated Energy $=-360.7344347$ (hartree), $-1508.5914059154\left(\mathrm{~kJ} \mathrm{~mol}^{-1}\right)$. Structure definition (3D Coordinates: Atom, X coord/Å, Y coord/Å, Z coord/Å): C, 1.2878997505, -0.3264218083, 0.0764760375; C, 0.0583739503, -0.0052213156, -0.8022554233; N, 0.0360941935, 0.0169724742, 2.079071246; C, 1.3345356595, -0.3537498458, 1.2472027595; O, -0.9599844531, 0.2417174049, 0.0770662408; O, 1.3998067633, -0.384047902, 2.4122063192; H, 2.2008255597, -0.5516769575, 0.6236863452; C, -2.2422486633, 0.5746383971, -0.4594925275; H, -0.8806881356, 0.2544210199, 2.4660337618; H, -2.8913763751, 0.7286520268, 0.4032138305; H, -2.1947954974, 1.4951756308, 1.0528915426; H, -2.6365675706, -0.2422076266, -1.0751565662. Harmonic Frequencies (Number, Frequency 
$\left(\mathrm{cm}^{-1}\right)$, IR Intensity $\left.\left(\mathrm{km} \mathrm{mol}^{-1}\right)\right): 1,80.2307,0.0646 ; 2,116.5802,3.0933 ; 3,145.0427,6.5499 ; 4,200.5356$, 0.3391; 5, 308.9482, 16.9389; 6, 414.4191, 8.2078; 7, 517.8895, 37.5935; 8, 530.4982, 17.1343; 9, 533.0285, 54.1011; 10, 703.7259, 27.7011; 11, 707.3444, 16.7011; 12, 788.5273, 132.0585; 13, 834.0951, 52.1989; 14, 1053.5166, 108.3110; 15, 1114.2231, 127.1478; 16, 1131.3229, 195.7939; 17, 1178.1218, 1.8116; 18, 1220.2034, 40.9133; 19, 1320.4151, 251.3309; 20, 1408.631, 133.3942; 21, 1492.7182, 33.2519; 22, 1512.6565, 8.6442; 23, 1524.6119, 5.7473; 24, 1694.5682, 639.8845; 25, 2197.1232, 1416.9063; 26, 3043.7072, 28.9904; 27, 3111.6487, 31.3994; 28, 3156.7347, 74.0808; 29, 3171.8191, 10.1735; 30, 3451.6613, 30.6312

\section{Calculation summary for $\mathbf{1 , 3}$ Migration of $\mathrm{OCH}_{3} 1 \mathrm{sZ}$ iE (acetonitrile)}

Geometry optimisation and frequency calculation: Program version = x86-Linux-G03RevB.01. Method and basis set $=$ RB3LYP/6-31G(d). Calculated Energy = -360.592337 (hartree), $-1507.997153334\left(\mathrm{~kJ} \mathrm{~mol}^{-1}\right)$. Higher level single-point calculation: Program = x86-Linux-G03RevB.01. Method and basis set $=$ RB3LYP/6311++G(3df,2p). Calculated Energy = -360.7349147 (hartree), $-1508.5934132754\left(\mathrm{~kJ} \mathrm{~mol}^{-1}\right)$. Structure definition (3D Coordinates: Atom, X coord/Å, Y coord/A, Z coord/ $\AA$ ): C, 1.0378277261, 0.0038414477, 0.3651215734; C, 1.2138778041, -0.1219361239, 1.6700076807; H, 1.9409049753, 0.1003887462, 0.2378795267; O, 1.3805914752, -0.232107621, 2.8198457594; C, -0.2836115984, 0.0180787391, 0.2860392328; N, -1.4581959577, -0.0778570568, 0.2094416885; O, -0.0973824761, 0.1605096733, 1.6237663166; C, -1.279608138, 0.1962692373, -2.4318412722; H, -1.435280397, -0.180027252, 1.2320582449; H, -0.9256097457, 0.3139391574, -3.4574283157; H, -1.8501236925, -0.7312974307, 2.3270600314; H, -1.919106192, 1.0372599573, -2.1479102277. Harmonic Frequencies (Number, Frequency $\left(\mathrm{cm}^{-1}\right)$, IR Intensity $\left.\left(\mathrm{km} \mathrm{mol}^{-1}\right)\right): 1,47.4805,2.4028 ; 2,125.9643,2.6156 ; 3,149.4482,26.1728 ; 4,191.1003$, 3.7070; 5, 299.9292, 19.6941; 6, 413.0799, 8.8237; 7, 509.8799, 10.5201; 8, 521.4662, 0.2610; 9, 535.7978, 35.1777; 10, 706.6689, 76.1269; 11, 722.8519, 4.5481; 12, 788.9756, 81.6875; 13, 925.1744, 52.9748; 14, 1003.3679, 60.1616; 15, 1116.4381, 202.5745; 16, 1134.4828, 170.2443; 17, 1182.952, 1.2919; 18, 1220.3446, 36.0146; 19, 1318.1839, 312.7690; 20, 1440.9299, 280.6026; 21, 1495.3191, 119.0748; 22, 1500.7915, 7.1536; 23, 1519.5611, 11.5149; 24, 1679.7266, 314.7749; 25, 2196.911, 1297.7365; 26, 3064.5151, 36.2829; 27, 3126.8341, 75.3270; 28, 3137.2155, 31.3739; 29, 3169.0654, 24.1696; 30, 3335.6293, 32.7417;

\section{Calculation summary for 1,3 Migration of $\mathrm{OCH}_{3} 1 \mathrm{sZ} \mathrm{iZ} \mathrm{(acetonitrile)}$}

Geometry optimisation and frequency calculation: Program version = x86-Linux-G03RevB.01. Method and basis set $=$ RB3LYP/6-31G(d). Calculated Energy = -360.588204 (hartree), $-1507.979869128\left(\mathrm{~kJ} \mathrm{~mol}^{-1}\right)$. Higher level single-point calculation: Program = x86-Linux-G03RevB.01. Method and basis set $=$ RB3LYP/6311++G(3df,2p). Calculated Energy = -360.7304315 (hartree), $-1508.574664533\left(\mathrm{~kJ} \mathrm{~mol}^{-1}\right)$. Structure definition (3D Coordinates: Atom, X coord/Å, Y coord/Å, Z coord/Å): C, 0.6684787696, 0.0965944602, 0.428396661; C, 0.6481495102, -0.149137249, 1.7318310835; H, 1.6170462608, 0.4261392683, 0.016210083; O, 0.6490994681, -0.3597861193, 2.8775501792; C, -0.5643733549, -0.0908568189, -0.3465062997; N, 1.6432945753, -0.4899328735, 0.2167408903; O, -0.5341807894, 0.1704257837, -1.683324174; C, 0.664243949, 0.6214566042, -2.3252476276; H, -2.3972045317, -0.5602260664, -0.4827640768; H, 0.4007303077, 0.7515243263, -3.3761877411; H, 0.9993826531, 1.5808409837, -1.9156907894; H, 1.4647646641, -0.1222076916, -2.2434046522. Harmonic Frequencies (Number, Frequency $\left(\mathrm{cm}^{-1}\right)$, IR Intensity $\left.\left(\mathrm{km} \mathrm{mol}^{-1}\right)\right): 1$, 43.6392, 0.0414; 2, 101.4769, 0.8340; 3, 130.2615, 1.4855; 4, 223.3873, 0.0806; 5, 280.1128, 1.2377; 6, 440.2765, 21.7909; 7, 502.6079, 15.2499; 8, 532.2796, 11.6245; 9, 546.0811, 56.8183; 10 , 616.2895, 1.9082; 11, 685.8997, 8.2769; 12, 812.8003, 176.0265; 13, 910.0355, 19.6786; 14, 1027.9399, 172.0837; 15, 1150.7929, 120.4345; 16, 1156.0223, 85.5168; 17, 1173.4409, 0.9967; 18, 1220.8544, 24.6866; 
19, 1286.8063, 384.9186; 20, 1449.1014, 314.1304; 21, 1504.9551, 108.1160; 22, 1518.1251, 7.8471; 23, 1524.5486, 11.7512; 24, 1674.2931, 404.9962; 25, 2199.9278, 1307.2839; 26, 3046.4735, 39.3769; 27, 3118.9725, 30.1407; 28, 3164.2591, 15.4901; 29, 3197.3041, 51.4158; 30, 3301.47, 69.4319;

\section{1,3 Migration of $\mathrm{OH}$}

\section{Calculation summary for 1,3 Migration of $\mathrm{OH} 3 \mathrm{sE}$ (vacuum)}

Geometry optimisation and frequency calculation: Program version = x86-Linux-G03RevB.01. Method and basis set = RB3LYP/6-31G(d). Calculated Energy = -321.2857149 (hartree), $-1343.6168597118\left(\mathrm{~kJ} \mathrm{~mol}^{-1}\right)$. Higher level single-point calculation: Program = x86-Linux-G03RevB.01. Method and basis set = RB3LYP/6$311+G(3 d f, 2 p)$. Calculated Energy = -321.4200419 (hartree), -1344.1786152258 ( $\left.\mathrm{kJ} \mathrm{mol}^{-1}\right)$. Structure definition (3D Coordinates: Atom, X coord/Å, Y coord/Å, Z coord/Å): C, 0.7945647293, 0.0685180594, 0.3851165513; C, 1.1710421253, -0.0543371724, 0.8802700166; N, 1.5239067422, -0.0395451267, 2.045828688; C, -0.6107610589, 0.0614083851, -0.8035704004; O, -1.4708133524, -0.0943283678, 0.2416881007; O, -0.9893439415, 0.1770000043, -1.9510285054; H, 1.543532783, 0.1639542663, 1.1624372191; H, -2.3632601439, -0.0741811133, -0.1498225039; H, 1.7045637417, -0.9278659909, 2.5166837557. Harmonic Frequencies (Number, Frequency $\left(\mathrm{cm}^{-1}\right)$, IR Intensity $\left(\mathrm{km} \mathrm{mol}^{-1}\right)$ ): 1, 101.6567, 2.3774; 2, 152.0905, 0.1865; 3, 410.2505, 3.9209; 4, 469.1051, 41.9226; 5, 506.4711, 50.2346; 6, 520.7888, 17.9074; 7, 592.0879, 79.4466; 8, 657.1598, 49.5771; 9, 779.891, 73.6348; 10, 854.2241, 60.3140; 11, 906.3066, 14.7209; 12, 958.1554, 386.2209; 13, 1137.0406, 50.6329; 14, 1208.279, 240.7102; 15, 1358.7616, 36.8441; 16, 1429.6031, 70.3775; 17, 1826.9511, 506.7818; 18, 2147.0762, 504.9441; 19, 3238.1165, 5.8767; 20, 3486.1892, 29.8508; 21, 3704.6694, 67.3657;

\section{Calculation summary for 1,3 Migration of $\mathrm{OH} 3 \mathrm{sZ}$ (vacuum)}

Geometry optimisation and frequency calculation: Program version = x86-Linux-G03RevB.01. Method and basis set = RB3LYP/6-31G(d). Calculated Energy = -321.2845608 (hartree), -1343.6120332656 $\left(\mathrm{kJ} \mathrm{mol}^{-1}\right)$. Higher level single-point calculation: Program = x86-Linux-G03RevB.01. Method and basis set = RB3LYP/6$311+G(3 d f, 2 p)$. Calculated Energy = -321.4189189 (hartree), $-1344.1739188398\left(\mathrm{~kJ} \mathrm{~mol}^{-1}\right)$. Structure definition (3D Coordinates: Atom, X coord/Å, Y coord/Å, Z coord/Å): C, 0.8196829826, 0.0268275998, 0.0663441599; C, 0.8277744199, 0.0562456568, 1.2597620312; H, 1.756460307, 0.049295917, 0.6116089429; N, 0.8338819689, 0.0832828037, 2.4751050112; C, -0.4543237683, -0.0013763492, 0.7939715464; O, -1.5670012174, -0.0253001258, -0.3060690809; O, -0.2407351152, -0.0102561131, 2.1381512078; H, -1.1280187549, -0.0363670801, -2.5414980944; H, 0.837473521, -0.8016399957, 2.9844563194. Harmonic Frequencies (Number, Frequency $\left(\mathrm{cm}^{-1}\right)$, IR Intensity $\left(\mathrm{km} \mathrm{mol}^{-1}\right)$ ): 1, 104.368, 3.5409; 2, 146.4123, 0.6598; 3, 403.2154, 5.9791; 4, 459.9937, 70.1576; 5, 497.5531, 28.5394; 6, 503.9726, 40.5204; 7, 623.0281, 71.6682; 8, 677.4845, 37.2160; 9, 777.9736, 76.2432; 10, 851.6774, 54.0246; 11, 943.3758, 119.6573; 12, 949.7058, 301.6726; 13, 1144.5065, 52.4358; 14, 1156.844, 358.9863; 15, 1329.9434, 0.3133; 16, 1471.6157, 176.5180; 17, 1813.3705, 284.2676; 18, 2156.2739, 535.4196; 19, 3229.6886, 5.0590; 20, 3492.6546, 35.3504; 21, 3700.7816, 68.3931;

\section{Calculation summary for 1,3 Migration of OH TS2 iE (vacuum)}

Geometry optimisation and frequency calculation: Program version = x86-Linux-G03RevB.01. Method and basis set = RB3LYP/6-31G(d). Calculated Energy = -321.2246398 (hartree), $-1343.3614436436\left(\mathrm{~kJ} \mathrm{~mol}^{-1}\right)$. Higher level single-point calculation: Program = x86-Linux-G03RevB.01. Method and basis set = RB3LYP/6$311+G(3 d f, 2 p)$. Calculated Energy = -321.3581846 (hartree), $-1343.9199279972\left(\mathrm{~kJ} \mathrm{~mol}^{-1}\right)$. Structure 
definition (3D Coordinates: Atom, X coord/Å, Y coord/Å, Z coord/Å): C, 0.7933101017, 0.082038457, 0.707905935; C, 0.8233804438, 0.0933107576, 0.6900512266; O, 1.5160469694, 0.0870888721, 1.6580298629; C, -0.5629482181, -0.0565408673, -0.9768288048; N, -1.6138929721, -0.2087606652, 1.5873269404; O, -0.7571786142, 0.1301458804, 0.8153175756; H, 1.6039256021, 0.1733804608, 1.4149380005; H, -1.0874285553, -0.6719936746, 1.2683692645; H, -1.6126470474, -0.4907902326, -

2.5608211097. Harmonic Frequencies (Number, Frequency $\left(\mathrm{cm}^{-1}\right)$, IR Intensity $\left(\mathrm{km} \mathrm{mol}^{-1}\right)$ ): 1, -491.2012 , 108.5538; 2, 148.4824, 3.4865; 3, 317.1724, 24.3978; 4, 386.984, 106.8171; 5, 500.2601, 50.9527; 6, 560.7523, 39.2013; 7, 591.9584, 64.2448; 8, 603.9834, 21.8851; 9, 673.1245, 82.8616; 10, 697.0238, 63.0200; 11, 822.7741, 46.2702; 12, 853.1313, 221.3530; 13, 1019.1518, 23.2403; 14, 1049.3633, 7.1955; 15, 1212.8537, 56.7885; 16, 1328.678, 13.0272; 17, 1951.7079, 1602.2094; 18, 2030.1451, 17.3642; 19, 3291.9915, 2.4576; 20, 3606.7353, 112.7698; 21, 3656.5644, 57.3781;

\section{Calculation summary for 1,3 Migration of OH TS2 iZ (vacuum)}

Geometry optimisation and frequency calculation: Program version = x86-Linux-G03RevB.01. Method and basis set = RB3LYP/6-31G(d). Calculated Energy = -321.229482 (hartree), -1343.381693724 (kJ mol $\left.{ }^{-1}\right)$. Higher level single-point calculation: Program = x86-Linux-G03RevB.01. Method and basis set = RB3LYP/6$311+G(3 d f, 2 p)$. Calculated Energy = -321.3627115 (hartree), -1343.938859493 $\left(\mathrm{kJ} \mathrm{mol}^{-1}\right)$. Structure definition (3D Coordinates: Atom, X coord/Å, Y coord/Å, Z coord/Å): C, 0.8813191168, 0.1363857292, -0.5833923073; C, 0.7769835611, -0.0254496805, 0.7949105028; N, 1.3108515557, -0.1894823033, 1.8878749188; C, 0.4395967067, 0.0872694292, -1.0089799378; O, -0.9972565296, 0.0175380919, 0.5648521525; O, 1.2117499161, 0.0825475138, -1.9070556492; H, 1.7740261104, 0.2948567267, -1.1692632417; H, 1.4045441018, -0.8593863412, 0.7125938404; H, 0.8143728399, -0.0990119753, 2.763943397. Harmonic

Frequencies (Number, Frequency $\left(\mathrm{cm}^{-1}\right)$, IR Intensity $\left.\left(\mathrm{km} \mathrm{mol}^{-1}\right)\right): 1,-482.4646,130.1495 ; 2,148.1527$, 3.7269; 3, 298.0122, 8.6510; 4, 417.5896, 155.0504; 5, 510.1701, 28.6324; 6, 551.9181, 51.4427; 7, 596.4316, 53.1626; 8, 611.3643, 26.9443; 9, 659.7496, 100.3944; 10, 676.8846, 55.3951; 11, 844.7539, 47.7986; 12 , 864.2723, 281.6362; 13, 973.3169, 28.5621; 14, 1049.5386, 10.2978; 15, 1202.2085, 56.6164; 16, 1343.6103, 11.8953; 17, 1969.8971, 1432.1019; 18, 2057.2049, 118.0647; 19, 3293.218, 3.5669; 20, 3641.588, 120.3704; 21, 3663.1956, 47.4659;

\section{Calculation summary for 1,3 Migration of $\mathrm{OH} 1 \mathrm{sE}$ iE (vacuum)}

Geometry optimisation and frequency calculation: Program version = x86-Linux-G03RevB.01. Method and basis set = RB3LYP/6-31G(d). Calculated Energy = -321.2800912 (hartree), -1343.5933413984 (kJ mol $\left.{ }^{-1}\right)$. Higher level single-point calculation: Program = x86-Linux-G03RevB.01. Method and basis set = RB3LYP/6$311+G(3 d f, 2 p)$. Calculated Energy = -321.4150308 (hartree), -1344.1576588056 (kJ mol $\left.{ }^{-1}\right)$. Structure definition (3D Coordinates: Atom, X coord/Å, Y coord/Å, Z coord/Å): C, 0.8761467157, 0.1274619622, 0.0979819133; C, 0.8479196436, -0.1129014454, 1.2074667421; O, 0.8382867933, -0.3218386503, 2.3527686128; C, -0.3503668475, 0.1193690517, -0.9000837278; N, -0.4895718682, 0.3280906405, 2.1495582201; O, -1.4446737, -0.1549303451, -0.139516365; H, 1.8376615524, 0.3303948098, 0.5538537756; H, -2.2016894237, -0.1385669852, -0.753587955; H, 0.399929132, 0.5221122441, 2.6080753173. Harmonic Frequencies (Number, Frequency $\left(\mathrm{cm}^{-1}\right)$, IR Intensity $\left(\mathrm{km} \mathrm{mol}^{-1}\right)$ ): 1, 95.6205, 0.0461; 2, 147.534, 0.9840; 3, 413.4496, 2.3579; 4, 506.4175, 28.5546; 5, 508.9748, 9.7896; 6, 525.5066, 111.2741; 7, 559.6619, 18.8751; 8, 666.5113, 17.4369; 9, 719.6751, 83.2088; 10, 807.9311, 58.0770; 11, 925.9288, 8.4817; 12, 1102.1839, 143.1948; 13, 1146.2795, 14.7124; 14, 1261.253, 170.5400; 15, 1386.2456, 16.0025; 16, 1447.4871, 142.6238; 17, 1739.5011, 384.9636; 18, 2238.2392, 775.6305; 19, 3239.3895, 8.8451; 20, 3510.3695, 2.4382; 21, 3698.9558, 61.9425; 


\section{Calculation summary for 1,3 Migration of $\mathrm{OH} 1 \mathrm{sE}$ iZ (vacuum)}

Geometry optimisation and frequency calculation: Program version = x86-Linux-G03RevB.01. Method and basis set = RB3LYP/6-31G(d). Calculated Energy = -321.2746288 (hartree), -1343.5704976416 (kJ mol $\left.{ }^{-1}\right)$. Higher level single-point calculation: Program = x86-Linux-G03RevB.01. Method and basis set = RB3LYP/6$311+G(3 d f, 2 p)$. Calculated Energy = -321.4099728 (hartree), $-1344.1365062496\left(\mathrm{~kJ} \mathrm{~mol}^{-1}\right)$. Structure definition (3D Coordinates: Atom, X coord/Å, Y coord/Å, Z coord/Å): C, 0.8637274434, -0.1141098716, 0.1617397512; C, -0.3942010232, 0.0028325856, -0.8939283041; N, -0.4224309458, -0.0584022754, 2.166305606; C, 0.9239031348, -0.0542349051, 1.1614987906; O, -1.4501672007, 0.1763042758, 0.0316243329; O, 1.0068162663, -0.0053055302, 2.3230180214; H, 1.7836857321, -0.2550763089, 0.715232511; H, -2.2702023866, 0.2503689458, -0.5464958587; H, -1.3702365795, 0.038606472, 2.5402663081. Harmonic Frequencies (Number, Frequency $\left(\mathrm{cm}^{-1}\right)$, IR Intensity $\left.\left(\mathrm{km} \mathrm{mol}^{-1}\right)\right): 1,102.5265$, 0.3108; 2, 146.1465, 2.5236; 3, 393.0935, 72.7278; 4, 420.737, 10.6189; 5, 510.6885, 28.0414; 6, 534.6173, 73.4120; 7, 536.3284, 12.9280; 8, 674.3218, 19.9912; 9, 714.7617, 17.2396; 10, 803.9961, 100.4488; 11, 903.0353, 26.7556; 12, 1098.9855, 123.2907; 13, 1134.0207, 27.5230; 14, 1198.5363, 201.0872; 15, 1368.7586, 9.3908; 16, 1450.7596, 60.9293; 17, 1745.7828, 382.7250; 18, 2238.8412, 775.9504; 19, 3249.7784, 18.3897; 20, 3451.889, 3.9380; 21, 3743.1108, 35.4246;

\section{Calculation summary for 1,3 Migration of OH $1 \mathrm{sZ}$ iE (vacuum)}

Geometry optimisation and frequency calculation: Program version = x86-Linux-G03RevB.01. Method and basis set = RB3LYP/6-31G(d). Calculated Energy = -321.2763141 (hartree), $-1343.5775455662\left(\mathrm{~kJ} \mathrm{~mol}^{-1}\right)$. Higher level single-point calculation: Program = x86-Linux-G03RevB.01. Method and basis set = RB3LYP/6$311+G(3 d f, 2 p)$. Calculated Energy = -321.4115398 (hartree), $-1344.1430594436\left(\mathrm{~kJ} \mathrm{~mol}^{-1}\right)$. Structure definition (3D Coordinates: Atom, X coord/Å, Y coord/A, Z coord/Å): C, 0.7996160857, 0.0660005957, 0.0673515143; C, 0.8995068501, 0.0419440355, 1.2520495626; H, 1.7189228689, 0.1535125874, 0.6363592555; O, 0.9847299017, 0.0205334085, 2.4148788453; C, -0.4885227485, -0.0203399529, 0.7753223962; N, -1.6831952098, -0.1262106014, -0.3397474115; O, -0.2732720805, 0.0283488704, 2.1152743465; H, -1.1597926277, -0.0327812966, -2.5172444552; H, -1.7320274659, -0.1539433817, 0.6787456882. Harmonic Frequencies (Number, Frequency $\left(\mathrm{cm}^{-1}\right)$, IR Intensity $\left(\mathrm{km} \mathrm{mol}^{-1}\right)$ ): 1, 62.6191, 4.2677; 2, 138.5201, 3.0293; 3, 413.9807, 3.2194; 4, 487.6026, 85.9822; 5, 509.9003, 18.1288; 6, 535.5449, 21.8441; 7, 590.7046, 40.6541; 8, 665.5496, 34.5586; 9, 716.673, 99.6966; 10, 799.9177, 22.5518; 11, 925.7202, 16.0350; 12, 1093.7918, 231.6396; 13, 1134.6225, 2.7375; 14, 1258.1096, 158.1384; 15, 1355.7284, 9.6616; 16, 1485.4366, 178.0470; 17, 1723.3703, 247.3154; 18, 2234.3559, 684.3100; 19, 3224.8207, 17.3501; 20, 3497.3556, 2.5976; 21, 3692.5477, 59.7120;

\section{Calculation summary for 1,3 Migration of $\mathrm{OH} 1 \mathrm{sZ}$ iZ (vacuum)}

Geometry optimisation and frequency calculation: Program version = x86-Linux-G03RevB.01. Method and basis set = RB3LYP/6-31G(d). Calculated Energy = -321.2709094 (hartree), $-1343.5549431108\left(\mathrm{~kJ} \mathrm{~mol}^{-1}\right)$. Higher level single-point calculation: Program = x86-Linux-G03RevB.01. Method and basis set = RB3LYP/6$311+G(3 d f, 2 p)$. Calculated Energy = -321.4067113 (hartree), -1344.1228666566 $\left(\mathrm{kJ} \mathrm{mol}^{-1}\right)$. Structure definition (3D Coordinates: Atom, X coord/A, Y coord/Å, Z coord/Å): C, 0.8137549458, 0.1072191, 0.0554617063; C, 0.8339953825, -0.0060470983, 1.2680289094; H, 1.7656712692, 0.2704619609, 0.5488433843; O, 0.8816116729, -0.101472068, 2.4257831468; C, -0.4647672259, 0.0095931011, 0.7754322241; N, -1.5623705171, -0.1805916704, -0.161065219; O, -0.3950017315, 0.1359766618, 2.1378308361; H, 0.5202726767, 0.2740677395, -2.4269504967; H, -2.3401284725, -0.2210153751, 0.8231779449. Harmonic Frequencies (Number, Frequency $\left(\mathrm{cm}^{-1}\right)$, IR Intensity $\left(\mathrm{km} \mathrm{mol}^{-1}\right)$ ): 1, 18.3834, 
7.0262; 2, 148.0619, 1.8754; 3, 245.171, 82.1628; 4, 415.6315, 9.8691; 5, 484.5822, 16.8559; 6, 489.2919, 70.8903; 7, 546.8598, 41.3186; 8, 656.0874, 19.2966; 9, 674.7408, 4.2616; 10, 848.4054, 84.5529; 11, 955.8309, 20.3953; 12, 1102.9539, 82.5918; 13, 1156.3739, 3.4194; 14, 1222.3586, 30.8391; 15, 1333.5511, 272.9346; 16, 1477.786, 230.6053; 17, 1750.3571, 214.0537; 18, 2245.5522, 668.9456; 19, 3219.9877, 3.5895; 20, 3483.8888, 3.3800; 21, 3749.4866, 28.6458;

\section{Calculation summary for 1,3 Migration of $\mathrm{OH} 3 \mathrm{sE}$ (acetonitrile)}

Geometry optimisation and frequency calculation: Program version $=$ x86-Linux-G03RevB.01. Method and basis set $=$ RB3LYP/6-311++G(d,p). Calculated Energy = -321.4229643 (hartree), -1344.1908367026 (kJ mol ${ }^{-}$

${ }^{1}$ ). Higher level single-point calculation: Program $=$ x86-Linux-G03RevB.01. Method and basis set = RB3LYP/6-311++G(3df,2p). Calculated Energy = -321.4448978 (hartree), -1344.2825625996 (kJ mol ${ }^{-1}$ ). Structure definition (3D Coordinates: Atom, X coord/Å, Y coord/Å, Z coord/Å): C, 0.232480433, 0.8485250256, 0.0075929146; C, 1.4361280162, -0.2904339613, 0.0046872306; N, 2.5302851976, 0.1980778545, -0.120680836; C, -0.997543438, -0.0704273994, 0.0041009651; O, -0.7969063685, 1.2635703528, -0.0000885786; O, -2.1166161187, -0.5607631871, 0.0031860744; H, 0.1527142358, 1.9319996269, 0.0270778818; H, -1.6789127193, 1.7127985644, -0.0117288961; H, 3.0959919306, 0.4665170747, 0.7063502381. Harmonic Frequencies (Number, Frequency $\left(\mathrm{cm}^{-1}\right)$, IR Intensity $\left(\mathrm{km} \mathrm{mol}^{-1}\right)$ ): 1 , 91.8416, 2.6179; 2, 150.438, 0.1145; 3, 414.1872, 34.4975; 4, 435.0504, 6.2791; 5, 466.024, 74.8291; 6, 489.5859, 218.8603; 7, 537.0586, 89.2835; 8, 675.9348, 55.2473; 9, 788.7515, 96.4869; 10, 811.4807, 109.0199; 11, 840.1301, 802.9126; 12, 910.9112, 63.8519; 13, 1121.2716, 89.9814; 14, 1167.2081, 351.3907; 15, 1346.5221, 87.8628; 16, 1413.8489, 164.6407; 17, 1692.3776, 1750.1670; 18, 2111.4031, 1151.0566; 19, 3114.6942, 114.2447; 20, 3133.9731, 567.8191; 21, 3292.3464, 454.7966;

\section{Calculation summary for 1,3 Migration of $\mathrm{OH} 3 \mathrm{sZ}$ (acetonitrile)}

Geometry optimisation and frequency calculation: Program version = x86-Linux-G03RevB.01. Method and basis set = RB3LYP/6-31G(d). Calculated Energy = -321.3084357 (hartree), $-1343.7118780974\left(\mathrm{~kJ} \mathrm{~mol}^{-1}\right)$. Higher level single-point calculation: Program = x86-Linux-G03RevB.01. Method and basis set = RB3LYP/6311++G(3df,2p). Calculated Energy = -321.4441067 (hartree), -1344.2792542194 (kJ mol $\left.{ }^{-1}\right)$. Structure definition (3D Coordinates: Atom, X coord/Å, Y coord/A, Z coord/Å): C, 0.8171147163, 0.0314280236, 0.0691127029; C, 0.8226082813, 0.0006773491, 1.2602326563; H, 1.7631497831, 0.0573164499, 0.6061057497; N, 0.834117714, 0.098394498, 2.4698551549; C, -0.4485657955, -0.00089107, -0.8009316228; O, -1.5664665034, -0.0377565593, -0.3022024273; O, -0.2428029205, 0.0165307235, -2.1360348679; H, 1.1365373344, -0.0036661623, -2.5711669179; H, 0.8617757309, -0.7598909029, 3.0530549605. Harmonic

Frequencies (Number, Frequency $\left(\mathrm{cm}^{-1}\right)$, IR Intensity $\left(\mathrm{km} \mathrm{mol}^{-1}\right)$ ): 1, 82.5594, 6.4290; 2, 143.2607, 5.9696; 3, 408.4038, 6.6737; 4, 459.3856, 125.2716; 5, 497.5952, 51.3215; 6, 500.9155, 65.7346; 7, 576.6536, 104.9791; 8, 678.5588, 54.8449; 9, 770.6661, 95.3734; 10, 831.2879, 84.4999; 11, 886.4923, 824.0053; 12, 948.886, 24.8058; 13, 1138.4799, 327.0021; 14, 1147.0791, 344.8920; 15, 1317.9912, 17.6503; 16, 1462.9206, 367.3487; 17, 1735.6466, 518.1994; 18, 2133.9802, 958.8508; 19, 3154.789, 88.8344; 20, 3169.054, 374.7408; 21, 3278.6107, 382.8634;

\section{Calculation summary for 1,3 Migration of OH TS2 iE (acetonitrile)}

Geometry optimisation and frequency calculation: Program version = x86-Linux-G03RevB.01. Method and basis set = RB3LYP/6-31G(d). Calculated Energy = -321.2489791 (hartree), -1343.4632305962 (kJ mol $\left.{ }^{-1}\right)$. Higher level single-point calculation: Program = x86-Linux-G03RevB.01. Method and basis set = RB3LYP/6$311++G(3 d f, 2 p)$. Calculated Energy = -321.3831758 (hartree), -1344.0244411956 $\left(\mathrm{kJ} \mathrm{mol}^{-1}\right)$. Structure 
definition (3D Coordinates: Atom, X coord/Å, Y coord/A, Z coord/Å): C, 0.7987917466, 0.0569683857, 0.7123106628; C, 0.804701881, 0.1024653514, 0.67995219; O, 1.5102762137, 0.1448258282, 1.6535677373; C, -0.5683747519, -0.0780376559, -0.9624050376; N, -1.6224918987, -0.2230541829, -1.5715183208; O, 0.7386220291, 0.1172059116, 0.8103170649; H, 1.6085430602, 0.1185704125, -1.4286764756; H, 1.0591974584, -0.7152196988, 1.2681702348; H, -1.5758490422, -0.426601839, -2.5813628692. Harmonic

Frequencies (Number, Frequency $\left(\mathrm{cm}^{-1}\right)$, IR Intensity $\left(\mathrm{km} \mathrm{mol}^{-1}\right)$ ): 1, -446.8186, 279.8833; 2, 147.1081, 4.2366; 3, 325.1993, 6.9943; 4, 410.2806, 140.9739; 5, 508.4841, 72.4876; 6, 568.5886, 75.6495; 7, 598.3426, 62.9558; 8, 609.8718, 107.3000; 9, 670.8312, 105.4576; 10, 717.299, 113.2696; 11, 830.035, 86.3587; 12, 863.1634, 378.3161; 13, 1021.5724, 18.2651; 14, 1054.3784, 64.7333; 15, 1215.3798, 81.2283; 16, 1315.7824, 22.1504; 17, 1841.6768, 3040.1285; 18, 1982.8654, 112.2956; 19, 3157.139, 450.1370; 20, 3227.7689, 26.9944; 21, 3266.4512, 516.4364;

\section{Calculation summary for 1,3 Migration of OH TS2 iZ (acetonitrile)}

Geometry optimisation and frequency calculation: Program version = x86-Linux-G03RevB.01. Method and basis set = RB3LYP/6-31G(d). Calculated Energy = -321.252719 (hartree), $-1343.478870858\left(\mathrm{~kJ} \mathrm{~mol}^{-1}\right)$. Higher level single-point calculation: Program = x86-Linux-G03RevB.01. Method and basis set $=$ RB3LYP/6311++G(3df,2p). Calculated Energy = -321.3863835 (hartree), -1344.037855797 (kJ mol $\left.{ }^{-1}\right)$. Structure definition (3D Coordinates: Atom, X coord/Å, Y coord/Å, Z coord/Å): C, 0.888058447, 0.1256208931, 0.6018976132; C, 0.7681054574, -0.020957041, 0.7854486246; N, 1.3019134087, -0.1438123786, 1.887177277; C, -0.4452308603, 0.0737391996, -0.9785736606; O, -0.9607026452, 0.0166793249, 0.5431975232; O, -1.2308720741, 0.0751117232, -1.8830201784; H, 1.7808727406, 0.2533838844, 1.2009866982; H, -1.3733842105, -0.8773322788, 0.7296153579; H, 0.7461170987, -0.1741116508, 2.7498475381. Harmonic Frequencies (Number, Frequency $\left(\mathrm{cm}^{-1}\right)$, IR Intensity $\left.\left(\mathrm{km} \mathrm{mol}^{-1}\right)\right): 1,-440.0144$, 312.8556; 2, 147.5695, 9.3206; 3, 308.7215, 6.4513; 4, 434.1188, 232.1882; 5, 514.4147, 30.4232; 6, 568.3727, 70.2080; 7, 606.8778, 44.0159; 8, 615.0759, 78.8780; 9, 668.2915, 58.7397; 10, 692.4939, 206.0280; 11, 856.6755, 368.5776; 12, 885.3781, 226.8316; 13, 1000.6597, 96.7853; 14, 1033.8988, 17.1146; 15, 1209.7811, 65.7201; 16, 1323.3539, 18.4818; 17, 1862.8465, 2941.0987; 18, 1995.512, 31.1169; 19, 3169.0507, 405.6742; 20, 3229.2633, 29.5980; 21, 3329.0985, 494.8304;

\section{Calculation summary for 1,3 Migration of $\mathrm{OH} 1 \mathrm{sE}$ iE (acetonitrile)}

Geometry optimisation and frequency calculation: Program version = x86-Linux-G03RevB.01. Method and basis set = RB3LYP/6-31G(d). Calculated Energy = -321.297558 (hartree), -1343.666387556 (kJ mol-1). Higher level single-point calculation: Program = x86-Linux-G03RevB.01. Method and basis set = RB3LYP/6311++G(3df,2p). Calculated Energy = -321.4335623 (hartree), -1344.2351575386 (kJ mol $\left.{ }^{-1}\right)$. Structure definition (3D Coordinates: Atom, X coord/A, Y coord/Å, Z coord/Å): C, 0.2431067254, -0.8551135515, 0.0021664753; C, 1.4455910795, -0.2977128673, 0.0013636875; O, 2.5062991883, 0.1882474188, 0.0007517477; C, -0.9729834888, -0.0358110217, -0.0005755265; N, -2.1850884047, -0.4397843153, 0.0024135009; O, -0.6829463926, 1.2905500691, -0.0007583907; H, 0.1875736323, -1.9423449961, 0.005068818; H, -1.540100684, 1.7891761592, -0.003715617; H, -2.2329623775, -1.4668962159, 0.0021333683. Harmonic Frequencies (Number, Frequency $\left(\mathrm{cm}^{-1}\right)$, IR Intensity $\left(\mathrm{km} \mathrm{mol}^{-1}\right)$ ): 1, 93.1876, 0.0885; 2, 126.0951, 5.7181; 3, 410.5654, 1.6904; 4, 464.9269, 206.8825; 5, 504.9015, 38.4975; 6, 527.39, 37.8080; 7, 541.7038, 1.2574; 8, 668.5525, 20.0669; 9, 713.601, 86.0126; 10, 797.9731, 98.6689; 11, 924.474, 26.9943; 12, 1105.1658, 225.8617; 13, 1135.0834, 66.9320; 14, 1249.2557, 239.2914; 15, 1380.7682, 39.6135; 16, 1438.3827, 284.7983; 17, 1699.4192, 795.3765; 18, 2193.6683, 1439.6041; 19, 3147.3335, 65.4332; 20 , 3315.4548, 305.2452; 21, 3329.7781, 65.5827; 


\section{Calculation summary for 1,3 Migration of $\mathrm{OH} 1 \mathrm{sZ}$ iE (acetonitrile)}

Geometry optimisation and frequency calculation: Program version = x86-Linux-G03RevB.01. Method and basis set = RB3LYP/6-31G(d). Calculated Energy = -321.293383 (hartree), -1343.648927706 (kJ mol $\left.{ }^{-1}\right)$. Higher level single-point calculation: Program = x86-Linux-G03RevB.01. Method and basis set = RB3LYP/6311++G(3df,2p). Calculated Energy = -321.4297825 (hartree), -1344.219350415 (kJ mol $\left.{ }^{-1}\right)$. Structure definition (3D Coordinates: Atom, X coord/Å, Y coord/Å, Z coord/Å): C, 0, 0.7876353487, 0.0643710299, 0.0658268402; C, 0, 0.8968232224, 0.0419428353, 1.2526507131; H, 0, 1.7182446943, 0.1516943628, 0.6267946787; O, 0, 1.0045768278, 0.0227089697, 2.4140466604; C, 0, -0.4962635302, -0.0210512677, 0.7793863681; N, 0, -1.6909874498, -0.1273972017, -0.3334026604; O, 0, -0.2712399764, 0.0290851048, 2.1155032267; H, 0, -1.1522704231, -0.0309098989, -2.565706033; H, 0, -1.7249271795, -0.154932233, 0.693346836. Harmonic Frequencies (Number, Frequency $\left(\mathrm{cm}^{-1}\right)$, IR Intensity $\left(\mathrm{km} \mathrm{mol}^{-1}\right)$ ): 1, 60.1513, 5.7758; 2, 129.5363, 4.2868; 3, 415.4867, 3.0169; 4, 455.115, 212.6187; 5, 511.4094, 19.9500; 6, 533.6705, 48.2103; 7, 544.0916, 2.8062; 8, 664.1357, 56.7921; 9, 707.1865, 105.9248; 10, 778.4486, 47.6938; 11, 922.6845, 23.3783; 12, 1093.1045, 492.0827; 13, 1127.7382, 8.2918; 14, 1256.6042, 216.3016; 15, 1354.2301, 23.2115; 16, 1474.4561, 339.7874; 17, 1685.9998, 424.2976; 18, 2197.9417, 1254.5959; 19, 3127.6695, 75.6556; 20, 3329.3659, 301.8635; 21, 3338.5349, 32.5666;

\section{Calculation summary for 1,3 Migration of OH 1 sE iZ (acetonitrile)}

Geometry optimisation and frequency calculation: Program version = DEC-AXP-OSF/1-G03RevB.05. Method and basis set = RB3LYP/6-31G(d). Calculated Energy = -321.2957472 (hartree), $-1343.6588147904\left(\mathrm{~kJ} \mathrm{~mol}^{-1}\right)$. Higher level single-point calculation: Program = DEC-AXP-OSF/1-G03RevB.05. Method and basis set = RB3LYP/6-311++G(3df,2p). Calculated Energy = -321.4315966 (hartree), -1344.2269369812 (kJ mol-1). Structure definition (3D Coordinates: Atom, X coord/A, Y coord/Å, Z coord/Å): C, -0.2681693388, 0.8539032521, 0.004808128; C, 0.9704075865, -0.0731287009, 0.0006351617; N, 2.1131603124, 0.6467163322, -0.0045058891; C, -1.4595462696, -0.2755256802, 0.0002165578; O, 0.7109526023, 1.2658412972, 0.003152592; O, -2.5181471385, 0.2171599329, -0.0034877859; H, -0.2295764162, 1.9410997841, 0.0105234696; H, 1.5679738815, 1.7648253651, -0.0045657644; H, 2.8708847689,

0.0546247031, -0.0056940151. Harmonic Frequencies (Number, Frequency $\left(\mathrm{cm}^{-1}\right)$, IR Intensity $\left(\mathrm{km} \mathrm{mol}^{-1}\right)$ ): 1, 97.4817, 0.2609; 2, 126.9363, 6.7974; 3, 409.4493, 145.7687; 4, 414.3873, 14.4897; 5, 506.3402, 29.9360; 6, 527.1281, 115.5643; 7, 532.6241, 6.0856; 8, 671.8522, 25.9950; 9, 708.7457, 20.9319; 10, 810.8228, 149.8627; 11, 908.6059, 54.1158; 12, 1107.4852, 108.9472; 13, 1138.1697, 103.6705; 14, 1187.8788, 314.2160; 15, 1368.646, 6.1575; 16, 1444.76, 120.3958; 17, 1701.806, 790.9431; 18, 2195.7348, 1421.4703; 19, 3157.2834, 70.8459; 20, 3274.2712, 45.6804; 21, 3310.6941, 318.8188;

\section{Calculation summary for 1,3 Migration of OH 1 sZ iZ (acetonitrile)}

Geometry optimisation and frequency calculation: Program version = x86-Linux-G03RevB.01. Method and basis set = RB3LYP/6-31G(d). Calculated Energy = -321.2917639 (hartree), -1343.6421566298 $\left(\mathrm{kJ} \mathrm{mol}^{-1}\right)$. Higher level single-point calculation: Program = x86-Linux-G03RevB.01. Method and basis set = RB3LYP/6-

311++G(3df,2p). Calculated Energy = -321.4283027 (hartree), -1344.2131618914 (kJ mol $\left.{ }^{-1}\right)$. Structure definition (3D Coordinates: Atom, X coord/Å, Y coord/Å, Z coord/Å): C, 0.8168931748, 0.107928156, 0.0561419558; C, 0.8425976088, -0.0046678422, 1.2639437874; H, 1.768333448, 0.2723253234, 0.5616359729; O, 0.8770014573, -0.1023380031, 2.4251524213; C, -0.4605351107, 0.0102235981, 0.7753679131; N, -1.5670047875, -0.1810554153, -0.1606694046; O, -0.3991311843, 0.1345170518, 2.1271325272; H, 0.5242168959, 0.2761541784, -2.45113202; H, -2.3402130532, -0.2194274559, 0.8413088386. Harmonic Frequencies (Number, Frequency $\left(\mathrm{cm}^{-1}\right)$, IR Intensity $\left(\mathrm{km} \mathrm{mol}^{-1}\right)$ ): 1, 63.5358, 
2.6474; 2, 132.4151, 2.7184; 3, 326.4498, 177.0570; 4, 417.4365, 14.5322; 5, 491.5438, 19.4735; 6, 511.7302, 64.5232; 7, 541.0891, 74.9647; 8, 660.7455, 22.5604; 9, 682.3249, 2.4607; 10, 824.4273, 120.8696; 11, 956.6418, 17.9524; 12, 1129.1032, 163.6247; 13, 1142.0229, 15.8881; 14, 1250.7947, 51.4048; 15, 1323.8529, 488.7523; 16, 1464.2177, 403.5704; 17, 1703.4143, 403.6081; 18, 2199.1138, 1226.4076; 19, 3131.3577, 62.2281; 20, 3301.0709, 60.0407; 21, 3348.8291, 314.8182;

\section{1,3 Migration of $\mathrm{SCH}_{3}$}

\section{Calculation summary for 1,3 Migration of $\mathrm{SCH}_{3} 3 \mathrm{sE}$ (vacuum)}

Geometry optimisation and frequency calculation: Program version = x86-Linux-G03RevB.01. Method and basis set = RB3LYP/6-31G(d). Calculated Energy = -683.5570818 (hartree), $-2858.6357160876\left(\mathrm{~kJ} \mathrm{~mol}^{-1}\right)$. Higher level single-point calculation: Program = x86-Linux-G03RevB.01. Method and basis set = RB3LYP/6311+G(3df,2p). Calculated Energy = -683.7014771 (hartree), -2859.2395772322 (kJ mol $\left.{ }^{-1}\right)$. Structure definition (3D Coordinates: Atom, X coord/A, Y coord/A, Z coord/Å): C, 1.5336841476, 0.0232209199, 0.4669147373; C, 1.9935227609, -0.0980267279, 0.7703664702; N, 2.4193776779, -0.0826186904, 1.9122848055; C, 0.1077423173, 0.0749660428, -0.8304351395; S, -1.0638456466, -0.0397709317, 0.5491215483; O, -0.2653884369, 0.1824293291, -1.9822108852; H, 2.2389808726, 0.0709842093, 1.2909629022; H, 2.5889101576, -0.9723196353, 2.3858432472; C, -2.6114285564, 0.066379076, 0.4132081341; H, -3.4294105174, -0.0227060013, 0.3050613192; H, -2.6705680941, 1.0246456586, 0.9316267806; H, -2.66004234, -0.7446089893, -1.1414169695. Harmonic Frequencies (Number, Frequency $\left(\mathrm{cm}^{-1}\right)$, IR Intensity $\left.\left(\mathrm{km} \mathrm{mol}^{-1}\right)\right): 1,38.4304,1.0460 ; 2,57.9953,0.7387 ; 3,112.9197,0.3413 ; 4,137.8273$, 7.1054; 5, 223.4455, 9.6302; 6, 351.5697, 3.7623; 7, 436.5902, 45.3536; 8, 451.6383, 0.0941; 9, 498.8682, 24.0219; 10, 604.3323, 36.2948; 11, 635.0007, 41.5098; 12, 707.4182, 39.1058; 13, 720.5512, 8.6623; 14, 856.627, 65.5594; 15, 958.178, 415.7201; 16, 995.8045, 4.2933; 17, 1000.2145, 3.7376; 18, 1100.5811, 146.0329; 19, 1185.9211, 132.7001; 20, 1382.6287, 1.7485; 21, 1395.0309, 20.6551; 22, 1489.8019, 7.8018; 23, 1501.4097, 12.3522; 24, 1756.9474, 337.1052; 25, 2136.2133, 510.1725; 26, 3085.4988, 13.5726; 27, 3178.5456, 3.6141; 28, 3182.2175, 3.5347; 29, 3214.346, 5.0093; 30, 3478.7649, 31.3310;

\section{Calculation summary for 1,3 Migration of $\mathrm{SCH}_{3} 3 \mathrm{sZ}$ (vacuum)}

Geometry optimisation and frequency calculation: Program version = x86-Linux-G03RevB.01. Method and basis set = RB3LYP/6-31G(d). Calculated Energy = -683.5584737 (hartree), -2858.6415370134 (kJ mol ${ }^{-1}$ ). Higher level single-point calculation: Program = x86-Linux-G03RevB.01. Method and basis set = RB3LYP/6-

311+G(3df,2p). Calculated Energy = -683.7024938 (hartree), -2859.2438290716 (kJ mol $\left.{ }^{-1}\right)$. Structure definition (3D Coordinates: Atom, X coord/A, Y coord/A, Z coord/Å): C, 1.1085470962, 0.0107506891, 0.7965403804; C, 1.0862481264, 0.0337943724, 2.1241984001; H, 2.0625981528, -0.0115589168, 0.2799470479; N, 1.062600351, 0.1827387696, 3.3308474536; C, -0.1504104512, -0.0071736845, 0.040979616; O, -1.2620137044, -0.0148301928, 0.5329795586; S, 0.1519393123, -0.0209818781, 1.7517463821; H, 1.0289036989, -0.6399821052, 3.9353251406; C, -1.5694382702, -0.0428534167, 2.3576207196; H, -2.2183007075, -0.038560535, -1.4772813919; H, -1.7403381648, -0.9455053289, 2.9474969687; H, -1.7556638048, 0.843679329, -2.9669064194. Harmonic Frequencies (Number, Frequency $\left(\mathrm{cm}^{-1}\right)$, IR Intensity $\left.\left(\mathrm{km} \mathrm{mol}^{-1}\right)\right): 1,69.5187,3.0241 ; 2,93.4271,0.7487 ; 3,119.4171,1.8089 ; 4,120.6545$, 5.3705; 5, 253.2942, 15.2345; 6, 320.7065, 2.7809; 7, 458.4765, 3.7026; 8, 468.5876, 49.5792; 9, 509.565, 12.9189; 10, 512.8598, 38.6819; 11, 689.1475, 29.3430; 12, 719.5034, 1.2306; 13, 845.6228, 60.0830; 14 , 855.5341, 177.8479; 15, 945.0985, 498.6886; 16, 999.3437, 9.9876; 17, 1005.4309, 3.4549; 18, 1030.9709, 219.7106; 19, 1156.8328, 1.1788; 20, 1371.7365, 9.8615; 21, 1428.7889, 140.0817; 22, 1510.0818, 10.3166; 23, 1511.48, 7.1101; 24, 1759.1798, 171.1051; 25, 2144.2869, 728.0781; 26, 3072.9536, 28.8400; 27, 
3166.4803, 1.1639; 28, 3170.7133, 6.8110; 29, 3217.8034, 3.9411; 30, 3487.6868, 34.8891;

\section{Calculation summary for 1,3 Migration of $\mathrm{SCH}_{3}$ TS2 iE (vacuum)}

Geometry optimisation and frequency calculation: Program version = x86-Linux-G03RevB.01. Method and basis set = RB3LYP/6-31G(d). Calculated Energy = -683.5158046 (hartree), -2858.4630948372 (kJ mol $\left.{ }^{-1}\right)$. Higher level single-point calculation: Program = x86-Linux-G03RevB.01. Method and basis set $=$ RB3LYP/6-

311+G(3df,2p). Calculated Energy = -683.6624605 (hartree), -2859.076409811 (kJ mol$\left.{ }^{-1}\right)$. Structure definition (3D Coordinates: Atom, X coord/A, Y coord/A, Z coord/Å): C, 1.1003604211, -0.1655794567, -1.0261983222; C, 1.2107536348, -0.1490604079, 0.3731494327; O, 2.0522572727, -0.0069352975, 1.2132560215; C, 0.2222935403, -0.3082187514, -1.431086985; N, -1.1206506856, -0.3781183648, -2.2598714527; S, 0.73122136, -0.3783772671, 0.7792477125; H, 1.9225507926, -0.0084648046, -1.7193232248; C, 1.1206313052, 1.3619874655, 1.1982394343; H, -1.0055406775, -0.7709963723, -3.1879071369; H, 2.188640805, 1.4345676351, 1.4138181278; H, -0.5478023655, 1.6425409569, 2.0859780405; H, 0.8636638298, 2.0239266948, 0.3679014306. Harmonic Frequencies (Number, Frequency ( $\mathrm{cm}^{-1}$ ), IR Intensity $\left.\left(\mathrm{km} \mathrm{mol}^{-1}\right)\right): 1$, -440.8196, 132.1566; 2, 62.2831, 0.3350; 3, 112.6418, 3.7813; 4, 131.853, 4.2618; 5, 162.4466, 1.7525; 6, 285.0875, 15.6868; 7, 355.5512, 48.8356; 8, 418.0359, 159.9156; 9, 518.288, 74.0155; 10, 561.5425, 34.2771; 11, 589.5596, 12.2033; 12, 619.5386, 21.6125; 13, 705.5905, 1.6427; 14, 715.3249, 74.0978; 15, 781.7987, 223.6592; 16, 986.4606, 2.9114; 17, 997.5996, 3.5282; 18, 1074.4462, 10.8320; 19, 1156.3029, 149.5488; 20, 1365.8724, 5.7756; 21, 1383.6327, 3.9536; 22, 1497.4445, 8.5065; 23, 1501.4368, 8.1614; 24, 1906.5826, 1171.8919; 25, 1992.3404, 347.4977; 26, 3073.6389, 13.8817; 27, 3163.3656, 1.7311; 28, 3172.7126, 2.2617; 29, 3201.5679, 2.3864; 30, 3582.2618, 100.5180;

\section{Calculation summary for 1,3 Migration of $\mathrm{SCH}_{3} \mathrm{TS} 2 \mathrm{iZ}$ (vacuum)}

Geometry optimisation and frequency calculation: Program version = x86-Linux-G03RevB.01. Method and basis set = RB3LYP/6-31G(d). Calculated Energy = -683.5245567 (hartree), -2858.4996961194 (kJ mol ${ }^{-1}$ ). Higher level single-point calculation: Program = x86-Linux-G03RevB.01. Method and basis set = RB3LYP/6311+G(3df,2p). Calculated Energy = -683.6702969 (hartree), -2859.1091816358 (kJ mol $\left.{ }^{-1}\right)$. Structure definition (3D Coordinates: Atom, X coord/Å, Y coord/A, Z coord/Å): C, 1.2917490327, 0.3620697771, 0.7947181085; C, 1.3303779451, 0.1949922961, 0.5874250572; N, 2.0521752946, -0.0371167954, 1.5577584374; C, -0.0293631792, 0.3335502299, -1.252806322; S, -0.8967201072, 0.3294874432, 0.583071925; O, -0.6603015983, 0.3147046358, -2.2676251623; H, 2.1743371324, 0.4594855365, 1.4190093326; C, -1.539566702, -1.3871239925, 0.6182769132; H, 1.7917527671, 0.1521922888, 2.5164352664; H, -2.3023887178, -1.4632832104, 1.3958576171; H, -1.9862577073, -1.6042815762, 0.3558060192; H, -0.7319186138, -2.0946615125, 0.8209986653. Harmonic Frequencies (Number, Frequency $\left(\mathrm{cm}^{-1}\right)$, IR Intensity $\left.\left(\mathrm{km} \mathrm{mol}^{-1}\right)\right): 1,-324.6308,143.1533 ; 2,96.2504,3.1945 ; 3,111.2704,1.4379 ; 4$, 137.0558, 1.9982; 5, 154.2549, 2.1271; 6, 272.8438, 12.0608; 7, 371.0896, 78.8552; 8, 449.0954, 61.0479; 9, 527.6874, 85.2405; 10, 567.7269, 38.6424; 11, 587.4462, 15.0571; 12, 626.9777, 2.2562; 13, 703.5269, 4.8096; 14, 730.2555, 49.0156; 15, 862.6463, 468.0109; 16, 980.7545, 1.1922; 17, 997.4126, 5.7155; 18, 1068.1084, 29.1454; 19, 1155.2788, 187.5757; 20, 1368.9503, 4.9437; 21, 1381.6807, 4.5110; 22, 1501.5149, 6.3106; 23, 1504.4081, 13.7635; 24, 1915.6399, 1278.9520; 25, 1975.2403, 39.6982; 26, 3072.22, 14.1540; 27, 3161.9834, 1.7784; 28, 3170.3383, 3.3102; 29, 3219.8875, 0.5067; 30, 3629.541, 100.6316;

\section{Calculation summary for 1,3 Migration of $\mathrm{SCH}_{3} 1 \mathrm{sE} \mathrm{iE} \mathrm{(vacuum)}$}

Geometry optimisation and frequency calculation: Program version = x86-Linux-G03RevB.01. Method and 
basis set = RB3LYP/6-31G(d). Calculated Energy = -683.5436873 (hartree), -2858.5797002886 (kJ mol $\left.{ }^{-1}\right)$. Higher level single-point calculation: Program = x86-Linux-G03RevB.01. Method and basis set = RB3LYP/6-

311+G(3df,2p). Calculated Energy = -683.6882855 (hartree), -2859.184409961 (kJ mol $\left.{ }^{-1}\right)$. Structure definition (3D Coordinates: Atom, X coord/Å, Y coord/A, Z coord/Å): C, 1.3772905692, 0.2438042993, -0.2872427472; C, 1.4454139786, 0.2589905901, 1.0375140187; O, 1.5208612988, 0.2564702579, 2.2014328683; C, 0.1205689099, 0.3712328951, -1.0460890026; N, 0.0258981417, 0.7519137203, -2.2612777885; S, 1.4397086876, 0.060644259, -0.1951930019; H, 2.3246629558, 0.2091337551, -0.8190425227; C, 1.1443295219, -1.5315809592, 0.6716821131; H, 0.942983281, 1.0249806902, -2.6317918883; H, 2.1337941492, -1.8644698976, 0.9967736681; H, -0.7335942644, -2.2764946429, -0.0132279946; H, 0.5067598189, -1.4332971049, 1.5526720488. Harmonic Frequencies (Number, Frequency (cm ${ }^{-1}$ ), IR Intensity $\left(\mathrm{km} \mathrm{mol}^{-1}\right)$ ): 1, 53.3248, 1.9960; 2, 101.6923, 4.6071; 3, 123.224, 2.8215; 4, 150.5472, 0.4961; 5, 225.6528, 0.0572; 6, 364.9782, 3.1812; 7, 409.2639, 6.4843; 8, 496.6062, 10.8799; 9, 535.7974, 15.1969; 10, 602.5935, 19.8038; 11, 646.4459, 13.7451; 12, 662.9124, 5.1459; 13, 705.1236, 1.3805; 14, 872.807, 91.9590; 15, 994.2261, 8.9463; 16, 1010.0047, 31.9538; 17, 1044.7887, 66.2403; 18, 1147.5532, 12.9979; 19, 1300.5334, 197.2784; 20, 1391.3099, 6.1913; 21, 1396.1679, 7.4641; 22, 1502.462, 13.1526; 23, 1515.1747, 13.7545; 24, 1658.414, 207.0908; 25, 2222.5092, 686.7105; 26, 3076.0262, 17.9458; 27, 3159.1785, 4.6974; 28, 3169.3053, 6.2051; 29, 3194.6887, 4.5820; 30, 3411.6863, 5.3507;

\section{Calculation summary for 1,3 Migration of $\mathrm{SCH}_{3} 1 \mathrm{sE}$ iZ (vacuum)}

Geometry optimisation and frequency calculation: Program version = x86-Linux-G03RevB.01. Method and basis set = RB3LYP/6-31G(d). Calculated Energy = -683.5480306 (hartree), -2858.5978639692 (kJ mol-1). Higher level single-point calculation: Program = x86-Linux-G03RevB.01. Method and basis set = RB3LYP/6-

311+G(3df,2p). Calculated Energy = -683.6923738 (hartree), -2859.2015072316 (kJ mol $\left.{ }^{-1}\right)$. Structure definition (3D Coordinates: Atom, X coord/Å, Y coord/Å, Z coord/Å): C, 0.7477503685, -1.2064984379, 0.3278586983; C, -0.6551826407, -0.9060235422, 0.0414548292; N, -1.532098578, -1.8224474635, 0.1747075778; C, 1.7622656862, -0.4293617808, -0.0267233139; S, -1.0580473464, 0.766286469, 0.5752697905; O, 2.6747803156, 0.242817066, -0.3038038072; H, 0.9890163635, -2.1565453629, 0.7961343525; C, -0.4130895301, 1.8553628697, 0.7581062672; H, -2.4687123963, -1.4630489417, 0.0256414746; H, -0.8135477804, 2.8492727952, 0.541576563; H, -0.7793151092, 1.5317025679, 1.7346034488; H, 0.6773006816, 1.9117565003, 0.7609422865. Harmonic Frequencies (Number, Frequency $\left(\mathrm{cm}^{-1}\right)$, IR Intensity $\left.\left(\mathrm{km} \mathrm{mol}^{-1}\right)\right): 1$, 48.5628, 1.1637; 2, 108.2874, 2.0192; 3, 120.4234, 1.0305; 4, 143.7303, 0.2849; 5, 206.9948, 1.0117; 6, 359.9104, 4.6412; 7, 400.9761, 9.4611; 8, 507.538, 18.1447; 9, 529.7626, 18.4050; 10, 596.9111, 15.7648; 11, 652.6888, 6.3941; 12, 678.7624, 37.8097; 13, 700.5112, 7.6189; 14, 876.6287, 48.2697; 15, 994.8902, 3.0798; 16, 1007.166, 28.2522; 17, 1047.5795, 91.5008; 18, 1134.2412, 6.3813; 19, 1300.1185, 171.2125; 20, 1393.7989, 1.8782; 21, 1403.8607, 19.5636; 22, 1502.0276, 13.4205; 23, 1511.9796, 12.7910; 24, 1670.3804, 203.0046; 25, 2224.9202, 700.3149; 26, 3076.554, 17.5381; 27, 3162.4625, 4.2056; 28, 3170.7409, 5.4391; 29, 3208.4568, 12.1113; 30, 3462.4703, 4.6925;

\section{Calculation summary for 1,3 Migration of $\mathrm{SCH}_{3} 1 \mathrm{sZ}$ iE (vacuum)}

Geometry optimisation and frequency calculation: Program version = x86-Linux-G03RevB.01. Method and basis set = RB3LYP/6-31G(d). Calculated Energy = -683.5512667 (hartree), -2858.6113973394 (kJ mol $\left.{ }^{-1}\right)$. Higher level single-point calculation: Program = x86-Linux-G03RevB.01. Method and basis set = RB3LYP/6311+G(3df,2p). Calculated Energy = -683.6965618 (hartree), -2859.2190214476 (kJ mol $\left.{ }^{-1}\right)$. Structure definition (3D Coordinates: Atom, X coord/A, Y coord/Å, Z coord/Å): C, 1.0974070001, -0.0780896796, 0.8006375868; C, 1.1274056662, -0.1994627901, 2.119028074; H, 2.0544936424, -0.0346609804, 
0.2901511039; O, 1.1309120046, -0.3063457906, 3.2809575789; C, -0.1690115953, -0.0035840983, 0.0424258381; N, -1.3757074045, -0.0382891127, 0.4616356948; S, 0.1565687123, 0.1576687113, 1.7110150412; C, -1.545021392, 0.2231819156, -2.3591346815; H, -1.4305204518, -0.1325048208, 1.4801967178; H, -1.4531682323, 0.3231365512, -3.4435953138; H, -2.0818739784, -0.6920789135, 2.1055898434; H, -2.0760526554, 1.0799268106, -1.9417734046. Harmonic Frequencies (Number, Frequency $\left(\mathrm{cm}^{-1}\right)$, IR Intensity $\left.\left(\mathrm{km} \mathrm{mol}^{-1}\right)\right): 1,25.9695,3.3663 ; 2,92.3639,10.3741 ; 3,115.149,1.4462 ; 4,122.4403$, 0.3659; 5, 238.5882, 8.9898; 6, 313.3596, 1.0012; 7, 462.1455, 5.3778; 8, 506.8271, 12.2336; 9, 530.8238, 16.4753; 10, 553.4885, 0.6984; 11, 628.8598, 13.3036; 12, 719.1305, 2.0003; 13, 807.4919, 81.2554; 14, 815.7121, 98.0870; 15, 963.561, 66.7648; 16, 999.8264, 5.5457; 17, 1014.13, 33.6748; 18, 1150.241, 2.2598; 19, 1254.3267, 103.7585; 20, 1383.7991, 6.3569; 21, 1425.2667, 149.4890; 22, 1483.1856, 7.9935; 23, 1499.9079, 14.4174; 24, 1659.0541, 118.9096; 25, 2222.6953, 851.5870; 26, 3083.3037, 12.6041; 27, 3175.7388, 4.8170; 28, 3184.0861, 2.9865; 29, 3212.5462, 12.4547; 30, 3428.5832, 3.7838;

\section{Calculation summary for 1,3 Migration of $\mathrm{SCH}_{3} 1 \mathrm{sZ} \mathrm{iZ} \mathrm{(vacuum)}$}

Geometry optimisation and frequency calculation: Program version = x86-Linux-G03RevB.01. Method and basis set = RB3LYP/6-31G(d). Calculated Energy = -683.5493706 (hartree), -2858.6034678492 $\left(\mathrm{kJ} \mathrm{mol}^{-1}\right)$. Higher level single-point calculation: Program = x86-Linux-G03RevB.01. Method and basis set = RB3LYP/6-

$311+G(3 d f, 2 p)$. Calculated Energy = -683.6945085 (hartree), -2859.210434547 (kJ mol $\left.{ }^{-1}\right)$. Structure definition (3D Coordinates: Atom, X coord/A, Y coord/A, Z coord/Å): C, 0.7107688438, -0.060072041, 0.7447755731; C, 0.7213694172, -0.0709587487, 2.0745854288; H, 1.6648202614, 0.0398236932, 0.2442212261; O, 0.7542760769, -0.0670247858, 3.2370457746; C, -0.5632598601, -0.2241674295, 0.0390070201; N, 1.6337074753, -0.4978375916, 0.6826047078; S, -0.5520745054, -0.0773723479, -1.7625348491; C, 0.9906259027, 0.8156080659, -2.1769575292; H, -2.4537800067, -0.5391044306, 0.0733539192; H, 0.9148101587, 1.052212255, -3.2415875629; H, 1.0741873524, 1.7483144145, -1.6147213259; H, 1.8778722111, 0.1953139806, -2.0237707797. Harmonic Frequencies (Number, Frequency (cm ${ }^{-1}$ ), IR Intensity $\left(\mathrm{km} \mathrm{mol}^{-1}\right)$ ): 1, 40.0775, 0.6823; 2, 62.8159, 0.4772; 3, 131.447, 0.5584; 4, 185.4164, 0.0929; 5, 219.5225, 0.5552; 6, 353.0771, 4.0228; 7, 409.7102, 8.8751; 8, 500.0922, 11.8976; 9, 506.7007, 7.8905; 10, 539.0413, 11.9458; 11, 636.6981, 41.2843; 12, 712.0163, 2.3295; 13, 812.1307, 76.7228; 14, 833.839, 52.6102; 15, 986.4079, 19.1202; 16, 987.4557, 23.8995; 17, 1029.0536, 20.9895; 18, 1155.0838, 1.2819; 19, 1253.2532, 213.4892; 20, 1387.9192, 0.6454; 21, 1439.4411, 179.9522; 22, 1501.0502, 12.7203; 23, 1514.2948, 17.0503; 24, 1655.23, 122.1806; 25, 2234.1397, 848.9988; 26, 3068.4546, 17.5741; 27, 3151.9008, 8.2473; 28, 3164.7271, 4.5517; 29, 3251.9761, 11.2823; 30, 3465.1091, 1.9991;

\section{Calculation summary for 1,3 Migration of $\mathrm{SCH}_{3} 3 \mathrm{sE}$ (acetonitrile)}

Geometry optimisation and frequency calculation: Program version = DEC-AXP-OSF/1-G03RevB.05. Method and basis set = RB3LYP/6-31G(d). Calculated Energy = -683.5724511 (hartree), $-2858.6999905002\left(\mathrm{~kJ} \mathrm{~mol}^{-1}\right)$. Higher level single-point calculation: Program = DEC-AXP-OSF/1-G03RevB.05. Method and basis set =

RB3LYP/6-311++G(3df,2p). Calculated Energy = -683.7173415 (hartree), -2859.305922153 (kJ mol $\left.{ }^{-1}\right)$.

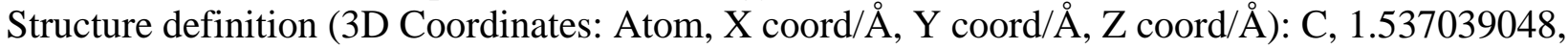
0.0362733945, -0.4703633158; C, 1.9852201227, -0.1029450812, 0.7754629457; N, 2.3955054995, 0.1048392687, 1.9168308871; C, 0.1233223626, 0.0785213185, -0.8349367418; S, -1.0577620956, 0.02853949, 0.5442412755; O, -0.2614350298, 0.1815613711, -1.9895612848; H, 2.2594263946, 0.1000349461, -1.2840215327; H, 2.5882786422, -1.0017079606, 2.4031821819; C, -2.6153261005, 0.0579040469, -0.4042536363; H, -3.423416701, 0.0092777802, 0.3293419242; H, -2.6711221876, 0.9962717651, -0.9586373992; H, -2.6875634745, -0.7843828519, -1.0945070244. Harmonic Frequencies 
(Number, Frequency ( $\mathrm{cm}^{-1}$ ), IR Intensity $\left(\mathrm{km} \mathrm{mol}^{-1}\right)$ ): 1, 16.2749, 4.0731; 2, 73.2366, 0.0500; 3, 110.9327, 0.5829; 4, 130.8021, 10.4785; 5, 219.6993, 18.8688; 6, 351.2027, 10.4976; 7, 436.1656, 80.2772; 8, 451.752, 0.1928; 9, 500.8697, 52.8674; 10, 597.9549, 121.5759; 11, 628.4914, 46.3084; 12, 699.7198, 60.9250; 13, 718.1297, 12.4818; 14, 820.0891, 113.6160; 15, 864.4105, 824.6352; 16, 993.2734, 5.2193; 17, 996.46, 10.8206; 18, 1097.1366, 228.7765; 19, 1177.0234, 216.0364; 20, 1376.4053, 3.0953; 21, 1397.9373, 53.3636; 22, 1482.6307, 10.3609; 23, 1492.3536, 18.1795; 24, 1704.9365, 889.4483; 25, 2125.3705, 970.4637; 26, 3080.3637, 11.0702; 27, 3135.8504, 65.3099; 28, 3162.2829, 475.2642; 29, 3173.5847, 2.4435; 30, 3177.7809, 3.1580;

\section{Calculation summary for 1,3 Migration of $\mathrm{SCH}_{3} 3 \mathrm{sZ}$ (acetonitrile)}

Geometry optimisation and frequency calculation: Program version = DEC-AXP-OSF/1-G03RevB.05. Method and basis set $=$ RB3LYP/6-31G(d). Calculated Energy = -683.5736828 (hartree), $-2858.7051414696\left(\mathrm{~kJ} \mathrm{~mol}^{-1}\right)$. Higher level single-point calculation: Program = DEC-AXP-OSF/1-G03RevB.05. Method and basis set = RB3LYP/6-311++G(3df,2p). Calculated Energy = -683.7180133 (hartree), -2859.3087316206 (kJ mol $\left.{ }^{-1}\right)$. Structure definition (3D Coordinates: Atom, X coord/Å, Y coord/Å, Z coord/Å): C, 1.143250285, 0.7407911755, -0.0035937198; C, 2.377478498, -0.2357507014, 0.0042595948; H, 1.0133320978, 1.8223593832, 0.0073509165; N, 3.4918023488, 0.2224236454, -0.1157138453; C, -0.0079443647, 0.1525691108, -0.0011075131; O, 0.0358885183, 1.3734074241, -0.0059909343; S, -1.5752973721, 0.7766816076, 0.0077620181; H, 4.0602228111, 0.4602510988, 0.7199918331; C, -2.7780744185, 0.5970998209, 0.0014101683; H, -2.2047837622, 1.5279659892, -0.0032812697; H, -3.4006597836, 0.5433268392, 0.8966962238; H, -3.4013379967, 0.5347339379, -0.8928367831. Harmonic Frequencies

(Number, Frequency $\left(\mathrm{cm}^{-1}\right)$, IR Intensity $\left(\mathrm{km} \mathrm{mol}^{-1}\right)$ ): 1, 17.6009, 4.5799; 2, 73.1669, 0.4954; 3, 113.6854, 12.6994; 4, 115.787, 1.0334; 5, 249.6242, 36.6983; 6, 318.9045, 5.6291; 7, 460.3476, 10.6575; 8, 470.9197, 88.9944; 9, 505.2915, 58.8578; 10, 514.7077, 61.8376; 11, 683.8519, 52.8916; 12, 715.8194, 0.9625; 13, 816.6809, 103.2963; 14, 837.6388, 966.8768; 15, 857.7686, 361.2577; 16, 994.377, 3.7773; 17, 1003.6304, 5.1122; 18, 1028.9828, 400.5439; 19, 1155.3119, 9.2594; 20, 1365.3464, 14.2476; 21, 1425.9109, 328.7415; 22, 1499.7023, 8.7954; 23, 1501.3517, 13.3252; 24, 1708.6725, 378.9519; 25, 2129.4087, 1378.8326; 26, 3072.6947, 25.9365; 27, 3139.6397, 68.4738; 28, 3162.191, 520.7805; 29, 3167.0013, 1.1670; 30, 3170.261, 4.0641 ;

\section{Calculation summary for 1,3 Migration of $\mathrm{SCH}_{3}$ TS2 iE (acetonitrile)}

Geometry optimisation and frequency calculation: Program version = x86-Linux-G03RevB.01. Method and basis set = RB3LYP/6-31G(d). Calculated Energy = -683.5312845 (hartree), -2858.527831779 $\left(\mathrm{kJ} \mathrm{mol}^{-1}\right)$. Higher level single-point calculation: Program = DEC-AXP-OSF/1-G03RevB.05. Method and basis set = RB3LYP/6-311++G(3df,2p). Calculated Energy = -683.678858 (hartree), -2859.144984156 (kJ mol ${ }^{-1}$ ). Structure definition (3D Coordinates: Atom, X coord/Å, Y coord/Å, Z coord/Å): C, 1.1309224363, 0.3399805804, -1.008952883; C, 1.2088837576, -0.0953630635, 0.3656219686; O, 2.0460972829, 0.1664566425, 1.1971386621; C, -0.2069505899, -0.4090580562, -1.4083146838; N, -1.1094964573, 0.4876056514, -2.2330672883; S, -0.6995002819, -0.3429680613, 0.7692366685; H, 1.9697860756, 0.4299600731, -1.698660687; C, -1.2141140052, 1.3643551153, 1.1887639957; H, -0.9520284489, 0.2030198221, -3.2134537349; H, -2.16899056, 1.3186495564, 1.7165044149; H, -0.453009536, 1.8027630487, 1.8403522935; H, -1.3185056763, 1.9609222, 0.2791223532. Harmonic Frequencies (Number, Frequency $\left(\mathrm{cm}^{-1}\right)$, IR Intensity $\left.\left(\mathrm{km} \mathrm{mol}^{-1}\right)\right): 1,-397.3962,272.2651 ; 2,84.7977,2.8435 ; 3,99.3667,12.0082$; 4, 132.5947, 3.2715; 5, 158.0564, 3.5253; 6, 287.9538, 13.4924; 7, 377.1191, 35.1150; 8, 422.0324, 320.0885; 9, 513.7085, 142.2401; 10, 571.7458, 69.5779; 11, 592.3277, 9.5546; 12, 620.5764, 30.9382; 13, 699.8413, 10.1316; 14, 710.8727, 138.6936; 15, 785.9785, 382.3705; 16, 975.6332, 3.4885; 17, 996.4848, 12.3674; 18, 
1055.7719, 29.1717; 19, 1151.8957, 249.5472; 20, 1351.1966, 20.2918; 21, 1373.9123, 5.7946; 22, 1485.1768, 8.7865; 23, 1492.5793, 17.9843; 24, 1810.9204, 2604.6238; 25, 1949.4045, 573.8038; 26, 3068.2901, 7.8449; 27, 3147.05, 8.2846; 28, 3159.4809, 0.6941; 29, 3170.6925, 0.6967; 30, 3231.0637, 652.8832;

\section{Calculation summary for 1,3 Migration of $\mathrm{SCH}_{3} \mathrm{TS} 2 \mathrm{iZ}$ (acetonitrile)}

Geometry optimisation and frequency calculation: Program version = x86-Linux-G03RevB.01. Method and basis set = RB3LYP/6-31G(d). Calculated Energy = -683.5383103 (hartree), -2858.5572136746 (kJ mol $\left.{ }^{-1}\right)$. Higher level single-point calculation: Program = DEC-AXP-OSF/1-G03RevB.05. Method and basis set = RB3LYP/6-311++G(3df,2p). Calculated Energy = -683.6855632 (hartree), -2859.1730253024 (kJ mol ${ }^{-1}$ ). Structure definition (3D Coordinates: Atom, X coord/Å, Y coord/Å, Z coord/Å): C, 1.2946849397, 0.33761327, -0.7891868739; C, 1.3166514745, 0.1977620774, 0.6050530988; N, 2.0273974475, 0.0217949745, 1.5904810498; C, -0.0239251351, 0.3232255869, -1.2403156225; S, -0.8934074072, 0.321286486, 0.5508104462; O, -0.6418863251, 0.3223364174, -2.2773709549; H, 2.1872450843, 0.4124478053, 1.4086526304; C, -1.5326992469, -1.3953092177, 0.6249039159; H, 1.7225193554, 0.1128879308, 2.5658402603; H, -2.1875527771, -1.4822653187, 1.4945052096; H, -2.104895216, -1.5875826437, 0.2869866129; H, -0.7077616569, -2.1070780099, 0.7051998152. Harmonic Frequencies (Number, Frequency

$\left(\mathrm{cm}^{-1}\right)$, IR Intensity $\left.\left(\mathrm{km} \mathrm{mol}^{-1}\right)\right): 1,-289.3124,364.8446 ; 2,79.9534,3.4096 ; 3,107.116,13.7927 ; 4$, 127.3015, 7.3322; 5, 157.685, 4.9280; 6, 280.205, 13.0894; 7, 356.7747, 184.9711; 8, 407.2092, 156.7470; 9, 517.7152, 110.2437; 10, 572.2375, 68.5485; 11, 585.8343, 21.4669; 12, 629.5823, 4.5919; 13, 698.9049, 10.8341; 14, 718.6181, 69.9405; 15, 809.9802, 939.9442; 16, 978.2907, 2.3351; 17, 995.3272, 11.8862; 18, 1053.8718, 60.7098; 19, 1150.9481, 290.1834; 20, 1358.6105, 14.8887; 21, 1376.0396, 5.4543; 22, 1486.9432, 8.9994; 23, 1490.3796, 18.4724; 24, 1823.5974, 2543.3949; 25, 1950.7352, 300.9146; 26, 3069.3584, 8.0184; 27, 3154.5836, 9.2271; 28, 3160.6064, 0.5599; 29, 3171.1327, 0.5723; 30, 3329.2578, 638.3187;

\section{Calculation summary for 1,3 Migration of $\mathrm{SCH}_{3} 1 \mathrm{sE}$ iE (acetonitrile)}

Geometry optimisation and frequency calculation: Program version = x86-Linux-G03RevB.01. Method and basis set = RB3LYP/6-31G(d). Calculated Energy = -683.5577989 (hartree), -2858.6387149998 (kJ mol-1). Higher level single-point calculation: Program = DEC-AXP-OSF/1-G03RevB.05. Method and basis set = RB3LYP/6-311++G(3df,2p). Calculated Energy = -683.7023725 (hartree), -2859.243321795 (kJ mol ${ }^{-1}$ ). Structure definition (3D Coordinates: Atom, X coord/Å, Y coord/Å, Z coord/Å): C, 1.3770669236, 0.261197749, -0.2907032476; C, 1.4519263655, 0.2487230494, 1.0329704638; O, 1.5307433221, 0.2275037218, 2.1968416964; C, 0.124614004, 0.3813559428, -1.0445048566; N, 0.0251073779, 0.7581028307, -2.2625772588; S, -1.4427487049, 0.0587267764, -0.1975866504; H, 2.3294584561, 0.2591380751, -0.8260642862; C, -1.1556051494, -1.5264252957, 0.6863429703; H, 0.9501047983, 1.0290659266, -2.6380642322; H, -2.1494718045, -1.8690498732, 0.9874481638; H, -0.7139802923, 2.2665408455, 0.0157337676; H, -0.5418429635, -1.4080999677, 1.581008254. Harmonic Frequencies (Number, Frequency (cm-1), IR Intensity $\left.\left(\mathrm{km} \mathrm{mol}^{-1}\right)\right): 1$, 59.8679, 3.5739; 2, 101.3913, 9.3110; 3, 120.0038, 4.9905; 4, 149.6794, 1.3250; 5, 219.1126, 0.2238; 6, 368.3967, 6.3408; 7, 400.0255, 2.3823; 8, 497.5516, 16.6749; 9, 532.7694, 28.0824; 10, 599.358, 47.1648; 11, 650.517, 21.4040; 12, 662.0352, 7.5919; 13, 700.7611, 3.3171; 14, 860.9333, 165.5068; 15, 992.4021, 13.6473; 16, 1005.6221, 52.2713; 17, 1051.936, 85.0143; 18, 1139.111, 34.3487; 19, 1305.0523, 382.7878; 20, 1388.6819, 13.3624; 21, 1392.8391, 21.6732; 22, 1491.9878, 17.5022; 23, 1505.3961, 22.4720; 24, 1636.9972, 508.3874; 25, 2188.8435, 1404.4238; 26, 3075.7098, 15.0490; 27, 3101.2994, 64.1558; 28, 3160.5058, 3.2118; 29, 3174.1706, 5.3584; 30, 3243.8447, 62.4536;

\section{Calculation summary for 1,3 Migration of $\mathrm{SCH}_{3} 1 \mathrm{sE}$ iZ (acetonitrile)}


Geometry optimisation and frequency calculation: Program version = x86-Linux-G03RevB.01. Method and basis set = RB3LYP/6-31G(d). Calculated Energy = -683.5588216 (hartree), -2858.6429919312 (kJ mol $\left.{ }^{-1}\right)$. Higher level single-point calculation: Program = DEC-AXP-OSF/1-G03RevB.05. Method and basis set =

RB3LYP/6-311++G(3df,2p). Calculated Energy = -683.7036484 (hartree), -2859.2486576088 $\left(\mathrm{kJ} \mathrm{mol}^{-1}\right)$. Structure definition (3D Coordinates: Atom, X coord/A,, Y coord/A, Z coord/Å): C, 0.7466400624, 1.197877614, 0.3389124048; C, -0.6543847297, -0.9161594024, 0.0296733452; N, -1.5235415826, 1.8488446697, 0.1124118344; C, 1.7604157849, -0.417624133, -0.0089165743; S, -1.085625802, 0.7625377947, -0.5468419522; O, 2.669056764, 0.2586099344, -0.290008552; H, 1.0048561909, 2.1426459073, 0.8209959105; C, -0.3693338369, 1.8637541997, 0.740065075; H, -2.4710199503, 1.4967805543, -0.0937665557; H, -0.8176829919, 2.8450102538, 0.5617067876; H, -0.6490539817, 1.5207692799, 1.7382606424; H, 0.715226848, 1.9535171228, 0.6570545218. Harmonic Frequencies

(Number, Frequency $\left(\mathrm{cm}^{-1}\right)$, IR Intensity $\left.\left(\mathrm{km} \mathrm{mol}^{-1}\right)\right): 1$, 49.5938, 3.1671; 2, 106.2808, 3.5465; 3, 117.8726, 2.1553; 4, 142.5152, 0.8427; 5, 209.3311, 2.0972; 6, 364.5493, 9.5088; 7, 398.4857, 6.6380; 8, 497.6278, 34.4849; 9, 528.8749, 28.0200; 10, 593.4431, 30.7143; 11, 652.5879, 7.7029; 12, 666.6658, 68.6477; 13, 696.9892, 8.5923; 14, 846.4784, 86.7545; 15, 992.7611, 4.0303; 16, 1003.4386, 57.2059; 17, 1046.0323, 165.9471; 18, 1126.3151, 12.7586; 19, 1291.0893, 324.3101; 20, 1388.0644, 2.7299; 21, 1402.7549, 45.1879; 22, 1492.0301, 18.3050; 23, 1502.8794, 22.3346; 24, 1647.3197, 479.6865; 25, 2191.495, 1410.5152; 26, 3075.4593, 15.1422; 27, 3113.2461, 69.7896; 28, 3161.4216, 3.2263; 29, 3174.4368, 4.6717; 30, 3280.0582, 105.2576;

\section{Calculation summary for $\mathbf{1 , 3}$ Migration of $\mathrm{SCH}_{3} 1 \mathrm{sZ} i E$ (acetonitrile)}

Geometry optimisation and frequency calculation: Program version = DEC-AXP-OSF/1-G03RevB.05. Method and basis set = RB3LYP/6-31G(d). Calculated Energy = -683.561905 (hartree), -2858.65588671 (kJ mol ${ }^{-1}$ ). Higher level single-point calculation: Program = DEC-AXP-OSF/1-G03RevB.05. Method and basis set = RB3LYP/6-311++G(3df,2p). Calculated Energy = -683.7072369 (hartree), -2859.2636647158 (kJ mol-1). Structure definition (3D Coordinates: Atom, X coord/Å, Y coord/Å, Z coord/Å): C, -1.16032369, 0.6864053383, 0.0150897413; C, -2.4046749104, -0.2321622882, -0.0120889885; H, -1.0536497486, 1.7725675968, 0.0360128889; O, -3.5001426052, 0.167496407, -0.0361344922; C, 0.0206800275, 0.1932844373, 0.0112776429; N, 0.1026790036, 1.4688018461, 0.0143417489; S, 1.5260022842, 0.7934532361, -0.0011812259; C, 2.8035586329, 0.505700578, 0.006165405; H, -0.8249348638, 1.9214857367, 0.0260050546; H, 3.7645939806, -0.0151765916, 0.0064304485; H, 2.7145683145, 1.131964053, -0.883099915; H, 2.7103332264, 1.1254576633, 0.8995720284. Harmonic Frequencies (Number, Frequency ( $\left.\mathrm{cm}^{-1}\right)$, IR Intensity $\left.\left(\mathrm{km} \mathrm{mol}^{-1}\right)\right): 1,27.6321,6.2343 ; 2,88.4655,18.6888 ; 3,118.2119$, 2.9164; 4, 127.2519, 0.3056; 5, 229.5948, 14.4011; 6, 312.2848, 2.3329; 7, 458.8793, 3.1212; 8, 504.9434, 18.0468; 9, 532.9465, 30.1283; 10, 549.9313, 6.0300; 11, 624.8234, 18.4161; 12, 717.0852, 5.6459; 13, 790.6398, 141.0202; 14, 805.495, 204.9346; 15, 963.2104, 113.8612; 16, 996.1939, 7.5723; 17, 1008.9307, 74.6374; 18, 1148.4466, 4.6491; 19, 1261.5359, 223.7344; 20, 1376.5, 10.5673; 21, 1424.7434, 269.5934; 22, 1476.6916, 10.7530; 23, 1492.3902, 21.6046; 24, 1644.9079, 245.6606; 25, 2188.8146, 1568.8423; 26, 3078.051, 11.3403; 27, 3109.3153, 70.3919; 28, 3170.4241, 4.2199; 29, 3177.7777, 3.6994; 30, 3270.5938, 33.3198;

\section{Calculation summary for 1,3 Migration of $\mathrm{SCH}_{3} 1 \mathrm{sZ}$ iZ (acetonitrile)}

Geometry optimisation and frequency calculation: Program version $=$ x86-Linux-G03RevB.01. Method and basis set = RB3LYP/6-31G(d). Calculated Energy = -683.5608868 (hartree), $-2858.6516285976\left(\mathrm{~kJ} \mathrm{~mol}^{-1}\right)$. Higher level single-point calculation: Program = DEC-AXP-OSF/1-G03RevB.05. Method and basis set = 
RB3LYP/6-311++G(3df,2p). Calculated Energy = -683.7063171 (hartree), -2859.2598181122 (kJ mol ${ }^{-1}$ ). Structure definition (3D Coordinates: Atom, X coord/A, Y coord/Å, Z coord/Å): C, 0.7158026391, 0.0561674279, 0.7408461859; C, 0.7338291548, -0.0818073465, 2.0688951625; H, 1.6728163098, 0.0730718648, 0.2467016763; O, 0.7555027081, -0.0943329972, 3.2331356768; C, -0.5602940241, 0.2309073901, 0.0435091738; N, -1.6268717001, -0.5459528592, 0.6804729651; S, -0.5723109403, 0.0237190682, -1.7519122778; C, 1.0043845907, 0.7984997688, -2.1856332785; H, -2.4517414144, 0.5921357973, 0.0629637601; H, 0.9253966111, 1.0298838903, -3.2515104722; H, 1.1306887817, 1.72941266, -1.6285121912; H, 1.861560831, 0.1379008391, -2.0331459607. Harmonic Frequencies (Number, Frequency $\left(\mathrm{cm}^{-1}\right)$, IR Intensity $\left.\left(\mathrm{km} \mathrm{mol}^{-1}\right)\right): 1,32.9163,0.9977 ; 2,59.2724,1.1378 ; 3,119.6213,2.8441 ; 4$, 188.9085, 0.1230; 5, 216.1928, 0.3607; 6, 353.2866, 6.1641; 7, 409.814, 8.0068; 8, 505.0113, 15.0339; 9, 509.0676, 13.6852; 10, 536.0072, 22.3957; 11, 635.2528, 71.7261; 12, 708.4382, 4.7358; 13, 789.9103, 129.4437; 14, 829.2433, 97.0027; 15, 983.6097, 83.4283; 16, 987.4076, 8.0399; 17, 1026.4687, 35.1222; 18, 1146.1731, 4.8211; 19, 1253.4578, 402.3321; 20, 1382.6636, 1.6038; 21, 1429.3195, 325.6248; 22, 1490.6577, 15.6041; 23, 1503.2207, 22.2431; 24, 1628.2729, 236.9364; 25, 2192.6532, 1541.1409; 26, 3067.8608, 11.6776; 27, 3154.485, 3.7017; 28, 3165.2882, 2.7888; 29, 3213.0658, 46.3019; 30, 3278.0554, 79.8064;

\section{1,3 Migration of $\mathrm{SH}$}

\section{Calculation summary for 1,3 Migration of SH 3 sE (vacuum)}

Geometry optimisation and frequency calculation: Program version = x86-Linux-G03RevB.01. Method and basis set = RB3LYP/6-31G(d). Calculated Energy = -644.2388434 (hartree), -2694.2068430988 (kJ mol $\left.{ }^{-1}\right)$. Higher level single-point calculation: Program = x86-Linux-G03RevB.01. Method and basis set = RB3LYP/6-

311+G(3df,2p). Calculated Energy = -644.3721604 (hartree), -2694.7643747928 (kJ mol $\left.{ }^{-1}\right)$. Structure definition (3D Coordinates: Atom, X coord/Å, Y coord/A, Z coord/Å): C, 1.1176552615, 0.0812024973, 0.5275152155; C, 1.5478349541, -0.045932106, 0.7205018546; N, 1.9375684889, -0.0365925647, 1.8740549957; C, -0.2973811983, 0.0850704023, -0.9243407891; S, -1.4953418641, -0.0693290319, 0.4527198987; O, -0.657281385, 0.1800409951, -2.0761341335; H, 1.8410153428, 0.1689925989, 1.3321159506; H, -2.5581978151, -0.0414580165, -0.3746003326; H, 2.129269852, -0.9244948414, 2.342010902. Harmonic Frequencies (Number, Frequency $\left(\mathrm{cm}^{-1}\right)$, IR Intensity $\left.\left(\mathrm{km} \mathrm{mol}^{-1}\right)\right)$ : 1, 85.241, 3.1605; 2, 124.6368, 0.1520; 3, 306.3346, 22.4475; 4, 332.6455, 4.3590; 5, 423.6171, 1.0463; 6, 444.3165, 45.5837; 7, 498.295, 24.7624; 8, 591.7569, 2.3775; 9, 609.9684, 107.8379; 10, 710.0322, 40.2270; 11, 855.5373, 69.2775; 12, 914.2874, 49.5581; 13, 948.4379, 377.0949; 14, 1103.6956, 125.3620; 15, 1186.2227, 128.4540; 16, 1398.2165, 22.0024; 17, 1784.7649, 401.0664; 18, 2139.3373, 482.6042; 19, 2716.9187, 1.1521; 20 , 3217.7046, 6.0470; 21, 3484.3781, 37.3812;

\section{Calculation summary for 1,3 Migration of SH 3 sZ (vacuum)}

Geometry optimisation and frequency calculation: Program version = x86-Linux-G03RevB.01. Method and basis set = RB3LYP/6-31G(d). Calculated Energy = -644.2388802 (hartree), -2694.2069969964 (kJ mol ${ }^{-1}$ ). Higher level single-point calculation: Program = x86-Linux-G03RevB.01. Method and basis set = RB3LYP/6-

311+G(3df,2p). Calculated Energy = -644.3722547 (hartree), -2694.7647691554 (kJ mol $\left.{ }^{-1}\right)$. Structure definition (3D Coordinates: Atom, X coord/A, Y coord/Å, Z coord/Å): C, 0.8425156196, 0.0305742432, 0.4301959784; C, 0.8129051029, 0.0466776368, 1.7586748569; H, 1.7992724687, 0.0554732003, 0.080765896; N, 0.7862543192, 0.0614107053, 2.9724003586; C, -0.4148697792, 0.0079861913, 0.3277230863; O, -1.5291475063, -0.01307728, 0.1463588276; S, -0.1161619506, -0.0018196397, 2.1318647287; H, -1.4281055619, -0.0410589274, -2.4376499771; H, 0.773518459, -0.8219851629, 3.4836919067. Harmonic Frequencies (Number, Frequency $\left(\mathrm{cm}^{-1}\right)$, IR Intensity $\left(\mathrm{km} \mathrm{mol}^{-1}\right)$ ): 1, 81.4867, 
3.0074; 2, 135.2363, 0.4152; 3, 291.2004, 7.9248; 4, 323.5141, 31.5721; 5, 426.6395, 0.9454; 6, 470.0383, 64.4852; 7, 489.5154, 6.7046; 8, 508.3039, 37.4568; 9, 691.9235, 32.4230; 10, 836.0973, 289.2168; 11, 839.0745, 61.9509; 12, 877.7053, 1.8682; 13, 930.4687, 414.1558; 14, 1058.6401, 181.9037; 15, 1157.6085, 3.1476; 16, 1428.2442, 150.4976; 17, 1782.1074, 211.0908; 18, 2149.8192, 698.8870; 19, 2717.3672, 1.8024; 20, 3219.1656, 3.8182; 21, 3498.4042, 45.6343;

\section{Calculation summary for 1,3 Migration of SH TS2 iE (vacuum)}

Geometry optimisation and frequency calculation: Program version = x86-Linux-G03RevB.01. Method and basis set = RB3LYP/6-31G(d). Calculated Energy = -644.1981253 (hartree), -2694.0365600046 (kJ mol $\left.{ }^{-1}\right)$. Higher level single-point calculation: Program = x86-Linux-G03RevB.01. Method and basis set = RB3LYP/6311+G(3df,2p). Calculated Energy = -644.3334191 (hartree), -2694.6023586762 (kJ mol $\left.{ }^{-1}\right)$. Structure definition (3D Coordinates: Atom, X coord/Å, Y coord/A, Z coord/Å): C, 0.9043382645, 0.0624428678, 0.8580545249; C, 1.0943155112, 0.0649601796, 0.5251392736; O, 1.950370444, 0.1462606053, 1.3459762103; C, -0.435094629, -0.05470107, -1.2148851976; N, -1.3518550104, -0.1120381038, 2.0197527823; S, -0.9258803321, -0.1096913008, 1.004904355; H, 1.7024999157, 0.2058963874, 1.5815629482; H, -1.1333346869, 1.2218164435, 1.1159892494; H, -1.2764132742, -0.4946819986, 2.9556334945. Harmonic Frequencies (Number, Frequency $\left(\mathrm{cm}^{-1}\right)$, IR Intensity $\left.\left(\mathrm{km} \mathrm{mol}^{-1}\right)\right): 1,-445.7305$, 130.1170; 2, 133.7888, 4.3036; 3, 256.0195, 21.0579; 4, 351.4135, 26.9090; 5, 407.8028, 170.5800; 6 , 425.3506, 28.3871; 7, 475.4888, 78.8506; 8, 505.2006, 20.5304; 9, 553.7328, 32.7517; 10, 613.0747, 24.5713; 11, 707.4177, 88.9570; 12, 744.0904, 33.4624; 13, 773.8368, 184.4317; 14, 1081.5743, 11.9046; 15, 1144.29, 157.6971; 16, 1377.6102, 2.9569; 17, 1953.0667, 1460.5030; 18, 2013.8551, 114.4459; 19, 2678.017, 6.0693; 20, 3201.9875, 0.9495; 21, 3591.85, 117.6665;

\section{Calculation summary for 1,3 Migration of SH TS2 iZ (vacuum)}

Geometry optimisation and frequency calculation: Program version = x86-Linux-G03RevB.01. Method and basis set = RB3LYP/6-31G(d). Calculated Energy = -644.2068449 (hartree), -2694.0730253718 (kJ mol $\left.{ }^{-1}\right)$. Higher level single-point calculation: Program = x86-Linux-G03RevB.01. Method and basis set = RB3LYP/6$311+G(3 d f, 2 p)$. Calculated Energy = -644.341224 (hartree), -2694.634998768 $\left(\mathrm{kJ} \mathrm{mol}^{-1}\right)$. Structure definition (3D Coordinates: Atom, X coord/Å, Y coord/Å, Z coord/Å): C, 1.0814066383, 0.1395869225, -0.6435397664; C, 1.0555032873, 0.0371289394, 0.74253278; N, 1.6970817577, 0.1247326797, 1.7811036754; C, 0.1921137348, 0.0917171309, -1.2045606977; S, -1.2025783415, -0.2987068559, 0.6123982707; O, 0.767261939, 0.1648310359, -2.2401158265; H, 1.9966144976, 0.2563691718, -1.2158640937; H, 1.6073787389, 0.9749775351, 0.8226532324; H, 1.4417637686, -0.2544120149, 2.6814455182. Harmonic

Frequencies (Number, Frequency $\left(\mathrm{cm}^{-1}\right)$, IR Intensity $\left(\mathrm{km} \mathrm{mol}^{-1}\right)$ ): 1, -367.0275, 130.8191; 2, 136.072, 1.8392; 3, 250.5428, 27.0894; 4, 322.4121, 89.7352; 5, 403.562, 63.0613; 6, 411.259, 6.2209; 7, 492.8302, 67.5756; 8, 501.7189, 75.4157; 9, 566.4299, 32.5647; 10, 611.008, 2.2720; 11, 714.0337, 37.8894; 12, 732.9508, 33.2332; 13, 817.2076, 468.6176; 14, 1077.4994, 28.8268; 15, 1140.9519, 194.6610; 16, 1384.1828, 4.3840; 17 , 1961.8005, 1360.9341; 18, 2016.1512, 36.2852; 19, 2671.7243, 7.6223; 20, 3216.0459, 0.0089; 21, 3657.7014, 149.2009;

\section{Calculation summary for 1,3 Migration of SH $1 \mathrm{sE}$ iE (vacuum)}

Geometry optimisation and frequency calculation: Program version = x86-Linux-G03RevB.01. Method and basis set = RB3LYP/6-31G(d). Calculated Energy = -644.2342679 (hartree), -2694.1877083578 (kJ mol $\left.{ }^{-1}\right)$. Higher level single-point calculation: Program = x86-Linux-G03RevB.01. Method and basis set = RB3LYP/6311+G(3df,2p). Calculated Energy = -644.3680856 (hartree), -2694.7473339792 (kJ mol $\left.{ }^{-1}\right)$. Structure 
definition (3D Coordinates: Atom, X coord/Å, Y coord/A, Z coord/Å): C, 1.1860190669, 0.2021548744, 0.1424449577; C, 1.2744462092, -0.0231572962, 1.162398506; O, 1.364220305, -0.2190515764, 2.3075087945; C, -0.0607998446, 0.1592054847, -0.9217197042; N, -0.1741988633, 0.3708008272, 2.1748983187; S, -1.536678789, -0.2330137674, 0.029047257; H, 2.1167649171, 0.4335503506, 0.6519463909; H, -2.3556778341, -0.160531621, -1.0383380303; H, 0.7334105566, 0.5827899932, 2.5996568809. Harmonic Frequencies (Number, Frequency $\left(\mathrm{cm}^{-1}\right)$, IR Intensity $\left(\mathrm{km} \mathrm{mol}^{-1}\right)$ ): 1, 28.6928, 0.0036; 2, 124.681, 0.9507; 3, 269.2017, 30.2002; 4, 339.9577, 7.9038; 5, 424.2302, 10.1456; 6, 471.373, 12.7133; 7, 534.2274, 15.5895; 8, 606.0851, 9.4556; 9, 634.7262, 9.1542; 10, 649.1864, 23.8038; 11, 835.7273, 103.4755; 12, 927.1902, 19.4873; 13, 1048.6508, 82.8953; 14, 1154.0115, 13.8172; 15, 1306.5089, 234.2044; 16, 1395.837, 13.2838; 17, 1669.8217, 225.9651; 18, 2228.4955, 749.5568; 19, 2716.6246, 0.2567; 20, 3207.108, 6.8527; 21, 3436.0958, 2.2643;

\section{Calculation summary for 1,3 Migration of SH $1 \mathrm{sE}$ iZ (vacuum)}

Geometry optimisation and frequency calculation: Program version = x86-Linux-G03RevB.01. Method and basis set = RB3LYP/6-31G(d). Calculated Energy = -644.2334389 (hartree), -2694.1842414798 (kJ mol $\left.{ }^{-1}\right)$. Higher level single-point calculation: Program = x86-Linux-G03RevB.01. Method and basis set = RB3LYP/6-

311+G(3df,2p). Calculated Energy = -644.3680769 (hartree), -2694.7472975958 (kJ mol $\left.{ }^{-1}\right)$. Structure definition (3D Coordinates: Atom, X coord/Å, Y coord/Å, Z coord/Å): C, 1.2005070897, -0.1198802225, 0.1830687854; C, -0.0389539737, -0.0301500158, -0.952843242; N, 0.0095401774, 0.0560116354, 2.2219572078; C, 1.2832034377, 0.02442054, 1.1332588937; S, -1.6100334837, 0.026557909, -0.0159293242; O, 1.3909273931, 0.1332826412, 2.2891680606; H, 2.1306014764, -0.2562883225, -0.7272954408; H, 1.3086185735, -0.9423117821, 0.8762881108; H, -0.9241868717, 0.0689891735, -2.6378487104. Harmonic

Frequencies (Number, Frequency $\left(\mathrm{cm}^{-1}\right)$, IR Intensity $\left(\mathrm{km} \mathrm{mol}^{-1}\right)$ ): 1, 108.1775, 2.5647; 2, 118.203, 2.6985; 3, 199.3773, 16.8730; 4, 351.1828, 2.9560; 5, 399.719, 12.5074; 6, 480.3029, 10.7135; 7, 524.7282, 10.5450; 8, 594.9689, 17.9135; 9, 630.8432, 32.9585; 10, 663.8706, 51.2381; 11, 846.5202, 52.8513; 12, 921.2617, 11.6465; 13, 1046.6141, 107.1389; 14, 1136.0948, 9.1781; 15, 1294.0632, 178.1136; 16, 1406.0866, 21.8054; 17, 1684.1571, 218.2783; 18, 2229.4653, 736.1357; 19, 2678.0775, 12.3216; 20, 3210.3558, 14.0355; 21, 3472.8213, 5.6090;

\section{Calculation summary for 1,3 Migration of SH 1 sZ iE (vacuum)}

Geometry optimisation and frequency calculation: Program version = x86-Linux-G03RevB.01. Method and basis set = RB3LYP/6-31G(d). Calculated Energy = -644.2327742 (hartree), -2694.1814617044 (kJ mol-1). Higher level single-point calculation: Program = x86-Linux-G03RevB.01. Method and basis set = RB3LYP/6-

311+G(3df,2p). Calculated Energy = -644.3667707 (hartree), -2694.7418350674 (kJ mol $\left.{ }^{-1}\right)$. Structure definition (3D Coordinates: Atom, X coord/A, Y coord/A, Z coord/Å): C, 0.8404449489, 0.0327627803, 0.4195574588; C, 0.8855811769, -0.0108242627, 1.743292609; H, 1.7912003378, 0.1044948084, 0.0988409772; O, 0.9050051852, -0.0503265206, 2.9082712533; C, -0.4328691505, -0.0131009696, 0.3263674778; N, -1.6327664869, -0.0957420902, 0.0986924294; S, -0.1395839181, 0.0654238187, 2.0988368034; H, -1.4477877495, 0.0026618097, -2.4173295905; H, -1.6796878251, -0.1341562088, 1.1216468505. Harmonic Frequencies (Number, Frequency $\left(\mathrm{cm}^{-1}\right)$, IR Intensity $\left(\mathrm{km} \mathrm{mol}^{-1}\right)$ ): 1, 25.644, 6.3028; 2, 129.7007, 2.9159; 3, 290.7483, 4.3716; 4, 294.3446, 39.9721; 5, 439.9733, 7.2886; 6, 509.0645, 8.5005; 7, 533.4049, 13.9598; 8, 540.1147, 0.9722; 9, 628.9899, 13.4086; 10, 812.6701, 108.8471; 11, 814.9467, 83.9679; 12, 886.6046, 28.9418; 13, 1016.1779, 71.1337; 14, 1152.5456, 2.1335; 15, 1256.5877, 105.9086; 16, 1426.9263, 157.0458; 17, 1668.9202, 138.3583; 18, 2225.4504, 790.6226; 19, 2713.6857, 0.6167; 20, 3215.0993, 13.1200; 21, 3425.5262, 3.2944; 


\section{Calculation summary for 1,3 Migration of SH 1 sZ iZ (vacuum)}

Geometry optimisation and frequency calculation: Program version = x86-Linux-G03RevB.01. Method and basis set = RB3LYP/6-31G(d). Calculated Energy = -644.2341944 (hartree), -2694.1874009808 $\left(\mathrm{kJ} \mathrm{mol}^{-1}\right)$. Higher level single-point calculation: Program = x86-Linux-G03RevB.01. Method and basis set = RB3LYP/6-

$311+G(3 d f, 2 p)$. Calculated Energy = -644.3688228 (hartree), -2694.7504169496 ( $\left.\mathrm{kJ} \mathrm{mol}^{-1}\right)$. Structure definition (3D Coordinates: Atom, X coord/Å, Y coord/Å, Z coord/Å): C, 0.8763660676, 0.0154920664, 0.3923738744; C, 0.9017843736, 0.0442643037, 1.7225727865; H, 1.8282434301, 0.0166015239, 0.1235993087; O, 0.9473508, 0.0833639551, 2.8833574275; C, -0.412015597, -0.0767932107, -0.2994783231; N, -1.4961967944, -0.2717941105, 0.3428943399; S, -0.3524626048, 0.0552401459, -2.109875471; H, 0.8287013668, 0.699165638, -2.2171033748; H, -2.3197810238, -0.2617413207, -0.2612196063. Harmonic

Frequencies (Number, Frequency $\left(\mathrm{cm}^{-1}\right)$, IR Intensity $\left(\mathrm{km} \mathrm{mol}^{-1}\right)$ ): 1, 66.4308, 0.2168; 2, 137.7948, 2.9975; 3, 180.6806, 21.5329; 4, 307.406, 0.5622; 5, 404.2518, 18.4048; 6, 502.7149, 17.2159; 7, 511.5989, 10.9944; 8, 538.0451, 10.5495; 9, 629.3972, 44.0078; 10, 806.1027, 89.5110; 11, 831.7281, 58.8905; 12, 900.4661, 6.5817; 13, 1035.3401, 40.8514; 14, 1154.1444, 2.3215; 15, 1253.1008, 206.7100; 16, 1436.5411, 158.8606; 17, 1675.4793, 132.6437; 18, 2236.1576, 797.4186; 19, 2691.0049, 13.7442; 20, 3243.845, 9.0416; 21, 3479.914, 3.0429;

\section{Calculation summary for 1,3 Migration of SH 3 sE (acetonitrile)}

Geometry optimisation and frequency calculation: Program version = x86-Linux-G03RevB.01. Method and basis set = RB3LYP/6-31G(d). Calculated Energy = -644.2578146 (hartree), -2694.2861806572 $\left(\mathrm{kJ} \mathrm{mol}^{-1}\right)$. Higher level single-point calculation: Program = DEC-AXP-OSF/1-G03RevB.05. Method and basis set =

RB3LYP/6-311++G(3df,2p). Calculated Energy = -644.3920946 (hartree), -2694.8477396172 (kJ mol-1). Structure definition (3D Coordinates: Atom, X coord/Å, Y coord/Å, Z coord/Å): C, 1.1152561324, 0.0848415856, -0.5314263423; C, 1.5424307769, -0.0418648202, 0.7237921133; N, 1.9295240856, 0.0299920682, 1.8720798659; C, -0.2898864447, 0.0857734301, -0.9213860898; S, -1.4899284729, 0.0838327724, 0.4509282642; O, -0.6608278444, 0.1927151005, -2.0772913886; H, 1.8511105799, 0.1751762321, -1.3303524452; H, -2.582816529, -0.0203190807, -0.3638310119; H, 2.1437128846, -

0.9178102919, 2.3672251894. Harmonic Frequencies (Number, Frequency $\left(\mathrm{cm}^{-1}\right)$, IR Intensity $\left(\mathrm{km} \mathrm{mol}^{-1}\right)$ ): 1 , 68.356, 5.7164; 2, 117.6968, 1.9640; 3, 279.9749, 43.4639; 4, 334.9351, 15.9752; 5, 425.8783, 1.9960; 6, 447.995, 89.8372; 7, 501.6202, 54.3250; 8, 594.4612, 43.1455; 9, 600.731, 175.6794; 10, 702.8063, 65.6877; 11, 821.143, 117.7349; 12, 855.6361, 857.1375; 13, 900.0663, 20.2682; 14, 1100.3726, 203.5461; 15, 1177.232, 207.9330; 16, 1400.8884, 55.6252; 17, 1716.3043, 1019.7489; 18, 2128.3636, 942.2814; 19, 2443.7057, 68.3715; 20, 3135.5033, 82.7322; 21, 3154.4066, 482.2354;

\section{Calculation summary for 1,3 Migration of SH 3 sZ (acetonitrile)}

Geometry optimisation and frequency calculation: Program version = x86-Linux-G03RevB.01. Method and basis set = RB3LYP/6-31G(d). Calculated Energy = -644.2586791 (hartree), -2694.2897959962 (kJ mol-1). Higher level single-point calculation: Program = DEC-AXP-OSF/1-G03RevB.05. Method and basis set = RB3LYP/6-311++G(3df,2p). Calculated Energy = -644.3929905 (hartree), -2694.851486271 (kJ mol $\left.{ }^{-1}\right)$. Structure definition (3D Coordinates: Atom, X coord/Å, Y coord/Å, Z coord/Å): C, 0.8434653906 , 0.0210221891, 0.4242263314; C, 0.8137227045, -0.0154376243, 1.7575952158; H, 1.8066858664, 0.0534566534, -0.0828878522; N, 0.77951263, 0.078848417, 2.9633778199; C, -0.4050300785, 0.0071598586, -0.3267701893; O, -1.5276462606, -0.0526657076, 0.1476672077; S, -0.1187691726, 0.0368857817, -2.129938474; H, -1.4431548643, 0.0013774378, -2.4600380645; H, 0.7884093341, 0.7661680928, 3.566650952. Harmonic Frequencies (Number, Frequency $\left(\mathrm{cm}^{-1}\right)$, IR Intensity $\left.\left(\mathrm{km} \mathrm{mol}^{-1}\right)\right): 1$, 
45.2936, 3.1799; 2, 131.9147, 4.0873; 3, 295.225, 23.8856; 4, 298.5514, 58.8226; 5, 429.62, 1.3919; 6, 474.9058, 91.8266; 7, 492.2825, 45.0820; 8, 514.4622, 63.3357; 9, 688.7177, 54.4351; 10, 815.3828, 112.0636; 11, 821.05, 1024.8656; 12, 850.7227, 385.2603; 13, 876.0188, 1.7018; 14, 1053.9374, 326.5922; 15, 1156.5122, 13.4588; 16, 1424.7315, 352.3309; 17, 1714.895, 436.7765; 18, 2131.4838, 1304.0423; 19, 2438.0275, 62.2255; 20, 3143.1153, 76.7146; 21, 3162.3198, 517.0053;

\section{Calculation summary for 1,3 Migration of SH TS2 iE (acetonitrile)}

Geometry optimisation and frequency calculation: Program version = x86-Linux-G03RevB.01. Method and basis set = RB3LYP/6-31G(d). Calculated Energy = -644.2159069 (hartree), -2694.1109226558 $\left(\mathrm{kJ} \mathrm{mol}^{-1}\right)$. Higher level single-point calculation: Program = DEC-AXP-OSF/1-G03RevB.05. Method and basis set = RB3LYP/6-311++G(3df,2p). Calculated Energy = -644.352086 (hartree), -2694.680423652 (kJ mol $\left.{ }^{-1}\right)$. Structure definition (3D Coordinates: Atom, X coord/Å, Y coord/Å, Z coord/Å): C, 0.9020557136 , 0.0451279306, -0.8602029217; C, 1.0648730369, 0.0910688832, 0.5198109244; O, 1.9371850141, 0.2042500567, 1.3393466517; C, -0.4427889699, -0.0832985019, -1.2224442882; N, -1.3699325629, 0.1700207026, -2.0055136614; S, -0.8960085047, -0.1097905124, 1.0099698792; H, 1.7021432319, 0.1511320783, -1.5929676622; H, -1.1500387235, 1.2313954439, 1.1433243612; H, -1.2687592899, 0.387124732, -3.0090346378. Harmonic Frequencies (Number, Frequency $\left(\mathrm{cm}^{-1}\right)$, IR Intensity $\left.\left(\mathrm{km} \mathrm{mol}^{-1}\right)\right): 1$, -410.5234, 425.1907; 2, 118.4959, 31.7108; 3, 233.0178, 249.2031; 4, 260.6058, 33.2394; 5, 360.3618, 160.8387; 6, 420.1313, 17.9999; 7, 477.4487, 181.4497; 8, 519.1068, 12.0063; 9, 550.0212, 77.5917; 10, 620.7231, 18.3234; 11, 690.813, 235.4814; 12, 716.5843, 174.6375; 13, 762.8903, 35.2202; 14, 1060.3484, 36.0256; 15, 1138.1837, 289.8981; 16, 1366.8392, 20.8707; 17, 1847.901, 2782.3677; 18, 1987.9112, 636.3671; 19, 2375.0218, 87.1625; 20, 3140.3514, 11.1895; 21, 3241.0944, 853.2822;

\section{Calculation summary for 1,3 Migration of SH TS2 iZ (acetonitrile)}

Geometry optimisation and frequency calculation: Program version = x86-Linux-G03RevB.01. Method and basis set = RB3LYP/6-31G(d). Calculated Energy = -644.2238209 (hartree), -2694.1440190038 (kJ mol $\left.{ }^{-1}\right)$. Higher level single-point calculation: Program = DEC-AXP-OSF/1-G03RevB.05. Method and basis set = RB3LYP/6-311++G(3df,2p). Calculated Energy = -644.3594585 (hartree), -2694.711255447 (kJ mol-1). Structure definition (3D Coordinates: Atom, X coord/Å, Y coord/Å, Z coord/Å): C, 1.0712033852, 0.1644121461, -0.6480155073; C, 1.0454989164, 0.0317600537, 0.7458055975; N, 1.7037908618, 0.0438393537, 1.7746082178; C, -0.2088613603, 0.0945610578, -1.1787955392; S, -1.1791064087, 0.284822888, 0.5964503253; O, -0.7876530117, 0.1663572284, -2.2278154645; H, 1.9823749101, 0.3307446674, -1.2213175012; H, -1.576467494, 1.0060955017, 0.8367534989; H, 1.3874375355, 0.1618068095, 2.7276576839. Harmonic Frequencies (Number, Frequency ( $\left.\mathrm{cm}^{-1}\right)$, IR Intensity $\left(\mathrm{km} \mathrm{mol}^{-1}\right)$ ): 1 , -307.7092, 423.7147; 2, 122.7327, 25.9642; 3, 249.4664, 186.9305; 4, 255.8762, 67.9635; 5, 365.8827, 146.5905; 6, 426.9453, 22.3328; 7, 480.2158, 159.4243; 8, 513.2, 15.2451; 9, 561.6231, 79.8667; 10, 617.6311, 5.9448; 11, 706.3674, 65.6887; 12, 748.235, 9.5985; 13, 766.8015, 929.8427; 14, 1057.5491, 58.1088; 15, 1137.1906, 325.8955; 16, 1371.6728, 13.5194; 17, 1861.004, 2719.7804; 18, 1980.6893, 267.4706; 19, 2373.0758, 84.1578; 20, 3149.4747, 13.3570; 21, 3339.4253, 758.9712;

\section{Calculation summary for 1,3 Migration of SH $1 \mathrm{sE}$ iE (acetonitrile)}

Geometry optimisation and frequency calculation: Program version = x86-Linux-G03RevB.01. Method and basis set = RB3LYP/6-31G(d). Calculated Energy = -644.2492828 (hartree), -2694.2505006696 (kJ mol $\left.{ }^{-1}\right)$. Higher level single-point calculation: Program = DEC-AXP-OSF/1-G03RevB.05. Method and basis set = RB3LYP/6-311++G(3df,2p). Calculated Energy = -644.3834908 (hartree), -2694.8117585256 (kJ mol ${ }^{-1}$ ). 
Structure definition (3D Coordinates: Atom, X coord/A, Y coord/Å, Z coord/Å): C, 1.1845739026, 0.2030631051, -0.1455622882; C, 1.2721746124, -0.0256475224, 1.1573212147; O, 1.3575165663, 0.2255938947, 2.3034888219; C, -0.0590103023, 0.1602772588, -0.9240526657; N, -0.1640152062, 0.3707424882, -2.1798296259; S, -1.5362764211, -0.2286363603, 0.0358707539; H, 2.1213248413, 0.4378720005, -0.6550182052; H, -2.3986928256, -0.1760304143, -1.0203472179; H, 0.7593353584, 0.5797368695, -2.5939073978. Harmonic Frequencies (Number, Frequency $\left(\mathrm{cm}^{-1}\right)$, IR Intensity $\left(\mathrm{km} \mathrm{mol}^{-1}\right)$ ): 1, -27.3332, 1.1999; 2, 115.7035, 4.6481; 3, 226.5781, 59.9300; 4, 338.6198, 15.6667; 5, 412.3772, 8.2582; 6, 477.6044, 18.3417; 7, 530.794, 27.8362; 8, 604.2968, 32.2486; 9, 638.7769, 15.1530; 10, 642.7914, 40.6032; 11, 826.6262, 164.1277; 12, 908.0298, 48.4244; 13, 1049.711, 117.6104; 14, 1142.8377, 34.0360; 15, 1305.288, 438.1076; 16, 1393.5226, 23.2929; 17, 1645.7721, 533.0770; 18, 2188.4073, 1494.3298; 19, 2443.8887, 64.0360; 20, 3108.8626, 71.7487; 21, 3255.2251, 68.7030;

\section{Calculation summary for 1,3 Migration of SH 1 sE iZ (acetonitrile)}

Geometry optimisation and frequency calculation: Program version = x86-Linux-G03RevB.01. Method and basis set = RB3LYP/6-31G(d). Calculated Energy = -644.2474415 (hartree), -2694.242800353 (kJ mol-1). Higher level single-point calculation: Program = DEC-AXP-OSF/1-G03RevB.05. Method and basis set = RB3LYP/6-311++G(3df,2p). Calculated Energy = -644.3825441 (hartree), -2694.8077994262 (kJ mol ${ }^{-1}$ ). Structure definition (3D Coordinates: Atom, X coord/Å, Y coord/A, Z coord/Å): C, 1.1985227377, 0.1360785932, -0.1821418732; C, -0.0389343713, -0.0376027387, -0.9516607491; N, 0.0040804277, 0.0600903002, -2.2229475483; C, 1.283289458, 0.0270703132, 1.1315799993; S, -1.6088048499, 0.0209747905, -0.0135136197; O, 1.3988640275, 0.1642626652, 2.284123781; H, 2.1398538488, 0.2894671899, -0.7131231266; H, -1.3374079444, -0.9869512123, 0.8700571341; H, -0.9383104664, 0.0857544425, -2.6397377647. Harmonic Frequencies (Number, Frequency $\left(\mathrm{cm}^{-1}\right)$, IR Intensity $\left(\mathrm{km} \mathrm{mol}^{-1}\right)$ ): 1, 107.4379, 2.8680; 2, 119.0626, 5.0847; 3, 257.444, 48.1774; 4, 354.2554, 8.0190; 5, 404.5365, 7.7661; 6, 478.4265, 19.0469; 7, 525.8587, 19.5183; 8, 593.482, 31.0801; 9, 624.626, 66.7682; 10, 655.8226, 68.6562; 11, 825.7128, 89.9109; 12, 908.4809, 16.4470; 13, 1046.7886, 201.1989; 14, 1127.947, 17.8144; 15, 1290.3534, 355.0042; 16, 1405.6298, 45.9406; 17, 1658.1549, 509.9097; 18, 2193.0925, 1460.4923; 19, 2417.8201, 36.4381; 20, 3113.8126, 68.5798; 21, 3291.396, 106.1448;

\section{Calculation summary for 1,3 Migration of SH 1 sZ iE (acetonitrile)}

Geometry optimisation and frequency calculation: Program version = x86-Linux-G03RevB.01. Method and basis set = RB3LYP/6-31G(d). Calculated Energy = -644.2474246 (hartree), -2694.2427296772 (kJ mol-1). Higher level single-point calculation: Program = DEC-AXP-OSF/1-G03RevB.05. Method and basis set =

RB3LYP/6-311++G(3df,2p). Calculated Energy = -644.381872 (hartree), -2694.804988704 (kJ mol $\left.{ }^{-1}\right)$. Structure definition (3D Coordinates: Atom, X coord/Å, Y coord/Å, Z coord/Å): C, 0.8206317811 , 0.0367172352, 0.4225199645; C, 0.8850382432, -0.0110030361, 1.7459597922; H, 1.7794735338, 0.114403085, -0.0925453934; O, 0.9442226499, -0.0529618052, 2.9091331423; C, -0.4466136218, 0.0113678667, -0.3228957629; N, -1.6511727044, -0.0928980794, 0.0955593725; S, -0.1358455869, 0.0623227912, -2.0989158978; H, -1.4489996086, 0.0040433067, -2.4662579509; H, -1.7068552184, 0.1277080486, 1.1259730013. Harmonic Frequencies (Number, Frequency ( $\left.\mathrm{cm}^{-1}\right)$, IR Intensity $\left(\mathrm{km} \mathrm{mol}^{-1}\right)$ ): 1 , 32.7065, 10.4602; 2, 137.1444, 3.8399; 3, 277.1494, 72.5663; 4, 292.1079, 5.9334; 5, 435.6986, 8.3195; 6, 507.0341, 13.6667; 7, 535.2569, 27.4536; 8, 543.2533, 5.3291; 9, 626.3248, 18.3759; 10, 797.1495, 139.1408; 11, 802.4166, 228.8133; 12, 879.1356, 57.0295; 13, 1012.4349, 127.3235; 14, 1151.2641, 5.7088; 15, 1267.7097, 219.7165; 16, 1425.8212, 297.6393; 17, 1647.0134, 270.3044; 18, 2191.3878, 1497.5738; 19, 2442.7613, 51.7850; 20, 3113.4587, 73.6424; 21, 3267.0716, 32.5728; 
Geometry optimisation and frequency calculation: Program version = x86-Linux-G03RevB.01. Method and basis set = RB3LYP/6-31G(d). Calculated Energy = -644.2493273 (hartree), -2694.2506867686 (kJ mol ${ }^{-1}$ ). Higher level single-point calculation: Program = DEC-AXP-OSF/1-G03RevB.05. Method and basis set =

RB3LYP/6-311++G(3df,2p). Calculated Energy = -644.3844955 (hartree), -2694.815960181 (kJ mol ${ }^{-1}$ ). Structure definition (3D Coordinates: Atom, X coord $/ \AA$, Y coord $/ \AA \AA$, Z coord/Å): C, 0.873950055 , 0.0988000451, 0.3886903471; C, 0.9251611602, -0.0185094148, 1.7107361422; H, 1.8124899952, 0.2896715925, -0.1279349187; O, 0.9781512355, -0.1206530628, 2.8698397781; C, -0.4153307419, 0.0318283757, -0.2947275728; N, -1.497807515, -0.2487059559, 0.3510069356; S, -0.3805659619, 0.1314053505, -2.0981071639; H, 0.9553678339, 0.3416667267, -2.2828673531; H, -2.3220425566, 0.3184312603, -0.2634433794. Harmonic Frequencies (Number, Frequency $\left(\mathrm{cm}^{-1}\right)$, IR Intensity $\left(\mathrm{km} \mathrm{mol}^{-1}\right)$ ): 1, 50.2802, 0.4197; 2, 123.5198, 3.0461; 3, 166.096, 54.3881; 4, 314.7064, 0.2957; 5, 406.8651, 20.4787; 6, 512.9315, 29.8151; 7, 514.9518, 13.4623; 8, 535.0386, 30.8449; 9, 636.2231, 57.0409; 10, 791.8187, 111.1327; 11, 819.6955, 126.0142; 12, 912.7205, 19.1718; 13, 1034.157, 88.0451; 14, 1150.7791, 1.7804; 15, 1257.2808, 421.0336; 16, 1428.6314, 296.3630; 17, 1645.1849, 254.0694; 18, 2191.4221, 1479.2752; 19, 2445.2818, 36.5338; 20, 3151.2693, 73.7493; 21, 3292.8991, 80.7467;

\section{Dimethylaminooxoketene 1,3 migation of $\mathrm{NMe}_{2}$}

\section{Calculation summary for dimethylaminooxoketene 1,3 migation of $\mathrm{NMe}_{2} 4 \mathrm{sE}$ (acetonitrile)}

Geometry optimisation and frequency calculation: Program version = x86-Linux-G03RevB.05. Method and basis set = RB3LYP/6-31G(d). Calculated Energy = -399.9276271 (hartree), -1672.4973365322 (kJ mol $\left.{ }^{-1}\right)$. Higher level single-point calculation: Program = x86-Linux-G03RevB.05. Method and basis set = RB3LYP/6311++G(3df,2p). Calculated Energy = -400.0801088 (hartree), -1673.1350150016 (kJ mol $\left.{ }^{-1}\right)$. Structure definition (3D Coordinates: Atom, X coord $/ \AA$, Y coord/Å, Z coord/Å): C, -1.4362355639, 0.2736494818, 0.1267915016; C, -1.5718906143, -0.6673947265, 1.0457540221; O, -1.7095494329, -1.4979641016, 1.8562703023; C, -0.2513459901, 0.5296335703, -0.7366704851; N, 0.9953003742, 0.2009318711, 0.2795587071; O, -0.4365235409, 1.0346647924, -1.8507124523; H, -2.3534274557, 0.7912201808, 0.1546238035; C, 2.1317524881, 0.3054450718, -1.1891786591; C, 1.3069046417, -0.2060169066, 1.084699123; H, 2.8034790371, 1.1167841604, -0.8801911214; H, 2.6973409528, -0.6339037656, 1.1880106802; H, 1.76281582, 0.5089590801, -2.1928939327; H, 2.3057277059, 0.1624815296, 1.3391529739; H, 0.604112865, 0.2400048521, 1.7921988104; H, 1.3063224765, -1.2975736067, 1.2084428889. Harmonic Frequencies (Number, Frequency $\left(\mathrm{cm}^{-1}\right)$, IR Intensity $\left(\mathrm{km} \mathrm{mol}^{-1}\right)$ ): 1, 48.2409, 2.5103; 2, 109.8608, 0.3021; 3, 119.7577, 7.6430; 4, 155.0158, 8.2990; 5, 187.976, 4.6628; 6, 217.9812, 8.8874; 7, 317.5743, 11.9212; 8, 405.9694, 5.6116; 9, 465.8372, 18.5932; 10, 491.2204, 16.5743; 11, 525.7826, 3.2625; 12, 553.6758, 46.0451; 13, 699.3149, 8.9111; 14, 721.2919, 58.3667; 15, 769.4685, 26.0814; 16 , 1000.2885, 20.0080; 17, 1085.4979, 12.5674; 18, 1118.5003, 119.2985; 19, 1135.0564, 3.2549; 20, 1173.0696, 5.1678; 21, 1202.0966, 152.6694; 22, 1287.9669, 59.4370; 23, 1390.5207, 27.6723; 24, 1422.616, 301.3196; 25, 1452.2714, 28.8816; 26, 1488.8722, 85.6607; 27, 1516.4977, 11.4542; 28, 1519.8089, 24.3034; 29, 1528.4879, 6.8843; 30, 1549.9941, 79.2424; 31, 1682.1974, 832.7609; 32, 2186.6546, 1259.4758; 33, 3033.7622, 40.5150; 34, 3041.4508, 57.6682; 35, 3092.262, 42.0159; 36, 3104.9826, 10.9285; 37, 3129.2415, 75.8537; 38, 3145.3177, 11.7741; 39, 3193.606, 3.1695;

\section{Calculation summary for dimethylaminooxoketene 1,3 migation of $\mathrm{NMe}_{2}$ Intermediate (acetonirile)}


basis set = RB3LYP/6-31G(d). Calculated Energy = -399.9193872 (hartree), -1672.4628772704 (kJ mol $\left.{ }^{-1}\right)$. Higher level single-point calculation: Program = x86-Linux-G03RevB.05. Method and basis set = RB3LYP/6-

311++G(3df,2p). Calculated Energy = -400.0701921 (hartree), -1673.0935433622 (kJ mol $\left.{ }^{-1}\right)$. Structure definition (3D Coordinates: Atom, X coord/A, Y coord/Å, Z coord/Å): H, 0.0001079018, -0.0000110212, 2.705187185; C, 0.0000831238, -0.0000056135, -1.6219173077; N, 0.0000062407, 0.000002986, 0.5294053661; C, -0.0000129935, 1.0444629366, -0.682967891; O, -0.0001986105, 2.2433348697, 0.539145745; C, 0.0000567463, -1.0444627233, -0.6829565122; O, 0.0000501562, -2.2433342, 0.5391215054; C, -1.2304745493, 0.0000572812, 1.3536304119; C, 1.2305032263, -0.0000550924, 1.3536060241; H, -2.0995701234, 0.0000372272, 0.6920959947; H, -1.2422510354, 0.895310682, 1.9812387903; H, -1.2422689323, -0.8951756544, 1.98128175; H, 2.0995726159, -0.0000201396, 0.6920342501; H, 1.2423058884, -0.8953185408, 1.9812003279; H, 1.2423143127, 0.8951704554, 1.9812681607. Harmonic Frequencies (Number, Frequency $\left(\mathrm{cm}^{-1}\right)$, IR Intensity $\left(\mathrm{km} \mathrm{mol}^{-1}\right)$ ): 1, 139.1464, 14.4100; 2, 163.2371, 0.0002; 3, 166.5748, 0.2927; 4, 191.627, 0.0001; 5, 251.7512, 0.0584; 6, 261.4453, 3.3527; 7, 280.1607, 6.3752; 8, 422.3681, 4.0286; 9, 506.5272, 73.4579; 10, 630.6988, 1.9121; 11, 648.5252, 0.0241 ; 12, 704.7622, 0.0008; 13, 715.7814, 58.4645; 14, 737.7596, 15.0742; 15, 749.5702, 87.0674; 16, 954.6015, 193.7075; 17, 1024.9319, 13.1810; 18, 1040.3839, 11.9124; 19, 1111.7765, 0.0002; 20, 1209.4924, 9.1548; 21, 1225.6062, 1.3639; 22, 1244.4216, 103.0926; 23, 1294.3892, 2.6281; 24, 1299.2205, 6.0210; 25, 1447.2722, 1.9389; 26, 1477.9077, 4.6147; 27, 1502.2863, 0.0000; 28, 1504.3805, 12.8536; 29, 1518.9563, 23.4503; 30, 1523.0643, 20.8747; 31, 1790.7913, 2534.2154; 32, 1942.8205, 12.2184; 33, 3070.5043, 8.2968; 34, 3073.9637, 11.5539; 35, 3155.653, 0.0002; 36, 3157.7988, 6.7249; 37, 3167.0471, 3.1969; 38, 3168.2366, 4.6938; 39, 3230.0003, 18.3586;

\section{Calculation summary for dimethylaminooxoketene 1,3 migation of $\mathrm{NMe}_{2}$ Rotation TS (acetonitrile)}

Geometry optimisation and frequency calculation: Program version = x86-Linux-G03RevB.05. Method and basis set = RB3LYP/6-31G(d). Calculated Energy = -399.9125763 (hartree), -1672.4343940866 (kJ mol $\left.{ }^{-1}\right)$. Higher level single-point calculation: Program = x86-Linux-G03RevB.05. Method and basis set = RB3LYP/6-

311++G(3df,2p). Calculated Energy = -400.0630959 (hartree), -1673.0638670538 (kJ mol $\left.{ }^{-1}\right)$. Structure definition (3D Coordinates: Atom, X coord/Å, Y coord/Å, Z coord/Å): C, -1.5635899346, 0.2784018061, 0.1763614561; C, -1.2886868994, -1.0079294513, 0.1672041736; O, -1.3856303163, -2.1402164042, 0.4467387013; C, -0.3198529443, 0.971063706, -0.2661804374; N, 0.6813886163, -0.0743086189, 0.0647658846; O, -0.0360430894, 2.1246222077, -0.5368995778; H, -2.570613281, 0.6544023311, 0.3397271057; C, 1.5775074678, -0.3760691753, -1.0618657565; C, 1.4198019963, 0.2209903613, 1.3020433315; H, 2.2477123423, 0.466181319, -1.2839097941; H, 2.1799125083, -1.2535723426, 0.8088680296; H, 0.9810575242, -0.6013911367, -1.9506682137; H, 2.0831675337, 1.0888559755, 1.1811722265; H, 0.7102013652, 0.42400396, 2.10935043; H, 2.0211508238, -0.6523096829, 1.5715371757.

Harmonic Frequencies (Number, Frequency $\left(\mathrm{cm}^{-1}\right)$, IR Intensity $\left(\mathrm{km} \mathrm{mol}^{-1}\right)$ ): 1, -133.5447, 60.8626; 2 , 56.3517, 7.5257; 3, 133.0943, 4.3822; 4, 218.028, 0.1099; 5, 219.176, 5.2433; 6, 249.9515, 1.5328; 7, 293.962, 15.0609; 8, 346.0082, 127.9933; 9, 375.2111, 5.3453; 10, 531.0866, 21.0168; 11, 597.1298, 15.6847; 12, 660.6189, 2.3276; 13, 687.6317, 73.8560; 14, 744.8803, 10.1847; 15, 777.8253, 16.6079; 16, 974.3759, 122.6562; 17, 1060.5452, 16.2182; 18, 1067.2783, 16.1172; 19, 1113.5409, 2.1510; 20, 1201.4592, 11.6391; 21, 1207.4691, 23.8068; 22, 1222.9, 146.3806; 23, 1296.1838, 38.0815; 24, 1352.9006, 79.0715; 25, 1453.2924, 0.0173; 26, 1481.6655, 0.6945; 27, 1503.3894, 5.2803; 28, 1514.8702, 6.5205; 29, 1517.5576, 18.6216; 30, 1528.7063, 21.9419; 31, 1772.2107, 1455.9881; 32, 2117.3321, 1132.2517; 33, 3028.9805, 27.8653; 34, 3032.8729, 73.7503; 35, 3110.8958, 27.5342; 36, 3111.0226, 9.8615; 37, 3143.3857, 9.5235; 38, 3144.9706, 21.1529; 39, 3175.0387, 31.5188;

Calculation summary for dimethylaminooxoketene 1,3 migation of $\mathrm{NMe}_{2} 4 \mathrm{sE}$ (vacuum) 
Geometry optimisation and frequency calculation: Program version = x86-Linux-G03RevB.05. Method and basis set = RB3LYP/6-31G(d). Calculated Energy = -399.9173691 (hartree), -1672.4544375762 (kJ mol $\left.{ }^{-1}\right)$. Higher level single-point calculation: Program = x86-Linux-G03RevB.05. Method and basis set = RB3LYP/6-

311++G(3df,2p). Calculated Energy = -400.0678622 (hartree), -1673.0837997204 (kJ mol $\left.{ }^{-1}\right)$. Structure definition (3D Coordinates: Atom, X coord/A, Y coord/Å, Z coord/Å): C, -1.2516515124, -0.265994068, 0.7391137517; C, -0.6825897534, 0.0762324927, 1.8819277844; O, -0.1803862943, 0.3820256798, 2.8925848889; C, -0.5628356682, -0.5063708946, -0.5661412486; N, 0.5983048386, 0.1811173309, 0.841300476; O, -1.0498700025, -1.3183658225, -1.3454723434; H, -2.2767192573, -0.6167931606, 0.7907516714 ; C, 1.3433628754, -0.1772797155, -2.0412718264; C, 1.0952961436, 1.3262024989, 0.0959672267; H, 1.2862659954, 0.6223319598, -2.7923862514; H, 2.3987207932, -0.3450735806, 1.7926642388; H, 0.9141119483, -1.0868709737, -2.4581974472; H, 1.5181732788, 2.0513375931, 0.8004862336; H, 0.282215751, 1.8245928212, 0.4358110597; H, 1.8816554857, 1.0566332852, 0.623407002.

Harmonic Frequencies (Number, Frequency ( $\mathrm{cm}^{-1}$ ), IR Intensity $\left(\mathrm{km} \mathrm{mol}^{-1}\right)$ ): 1, 49.8573, 1.2680; 2, 110.2753, 0.0924; 3, 113.9598, 4.0028; 4, 155.9464, 3.8932; 5, 191.4495, 2.8185; 6, 213.1221, 6.1359; 7, 319.4368, 5.5214; 8, 405.8231, 3.7761; 9, 462.3164, 11.6876; 10, 493.5929, 8.5397; 11, 530.7818, 1.2533; 12, 559.3176, 26.2656; 13, 699.9903, 5.0068; 14, 726.5459, 33.2924; 15, 771.9706, 19.8849; 16, 1002.7945, 14.3116; 17 , 1090.1173, 7.7678; 18, 1124.7983, 86.4056; 19, 1140.4478, 1.5277; 20, 1176.6011, 3.9364; 21, 1203.95 , 109.7900; 22, 1293.1649, 46.3161; 23, 1386.1648, 24.9937; 24, 1421.4098, 205.6866; 25, 1454.629, 11.8798; 26, 1492.33, 34.5317; 27, 1523.1216, 8.6427; 28, 1526.119, 20.6049; 29, 1536.7091, 6.6769; 30, 1558.5496, 31.2201; 31, 1748.7087, 403.7926; 32, 2216.658, 692.0317; 33, 3027.0481, 38.6854; 34, 3035.3732, 62.1362; 35, 3082.1352, 49.5264; 36, 3091.3341, 13.7117; 37, 3149.3487, 13.0578; 38, 3194.8012, 1.1362; 39, 3225.8042, 16.0544;

\section{Calculation summary for dimethylaminooxoketene 1,3 migation of $\mathrm{NMe}_{2}$ Intermediate (vacuum)}

Geometry optimisation and frequency calculation: Program version = x86-Linux-G03RevB.05. Method and basis set = RB3LYP/6-31G(d). Calculated Energy = -399.9072287 (hartree), -1672.4120304234 (kJ mol $\left.{ }^{-1}\right)$. Higher level single-point calculation: Program = x86-Linux-G03RevB.05. Method and basis set = RB3LYP/6$311++G(3 d f, 2 p)$. Calculated Energy = -400.056144 (hartree), $-1673.034794208\left(\mathrm{~kJ} \mathrm{~mol}^{-1}\right)$. Structure definition (3D Coordinates: Atom, X coord/A, Y coord/Å, Z coord/Å): H, 2.69879345, -0.0000002569, -0.0000032892; C, 1.61894845, -0.0000001633, -0.0000030668; N, -0.53923955, 0.0000000239, -0.0000026223; C, 0.6921096675, -0.0000099613, 1.0561291241; O, 0.5406309129, -0.0000210911, 2.2474441552; C, 0.6921092325, 0.0000097956, -1.0561348758; O, 0.5406299871, 0.0000209517, -2.2474488446; C, 1.3565421906, 1.2278416574, 0.0000166712; C, -1.3565429094, -1.2278413425, -0.0000055791; H, 0.6911549372, 2.0937014626, 0.0000293795; H, -1.9830959996, 1.2497497183, 0.8964729988; H, 1.9830963688, 1.2497779634, -0.896438001; H, -0.6911561628, -2.0937015372, -0.0000075615; H, 1.9830971004, -1.2497610365, -0.8964606487; H, -1.9830967312, -1.2497652816, 0.8964493513. Harmonic

Frequencies (Number, Frequency $\left(\mathrm{cm}^{-1}\right)$, IR Intensity $\left.\left(\mathrm{km} \mathrm{mol}^{-1}\right)\right): 1,138.0709,8.0605 ; 2,140.3463,0.2011$; 3, 152.3521, 0.0000; 4, 189.7294, 0.0000; 5, 233.9626, 2.1837; 6, 256.4447, 2.0284; 7, 268.627, 1.3451; 8, 418.8055, 0.6498; 9, 451.2305, 42.4882; 10, 627.0167, 2.8903; 11, 644.4342, 0.0008; 12, 701.8085, 0.0000; 13, 705.8563, 49.4770; 14, 737.3746, 0.9327; 15, 751.9725, 65.1130; 16, 954.0283, 137.6214; 17, 1027.4366, 3.4014; 18, 1051.0249, 7.8915; 19, 1113.3867, 0.0000; 20, 1215.8565, 8.2377; 21, 1225.7071, 1.6671; 22, 1238.4023, 81.8977; 23, 1296.0262, 1.8658; 24, 1310.2019, 6.9744; 25, 1449.1821, 1.3682; 26, 1481.04, 2.9651; 27, 1509.7423, 10.3575; 28, 1511.3364, 0.0000; 29, 1527.3396, 12.5039; 30, 1531.4705, 11.1838; 31, 1882.2165, 1275.8771; 32, 1985.2772, 1.9411; 33, 3068.661, 13.2529; 34, 3073.44, 21.8744; 35, 3149.5599, 0.0000; 36, 3151.2539, 13.9154; 37, 3167.757, 5.0776; 38, 3168.744, 8.3945; 39, 3293.1084, 0.1336; 
Calculation summary for dimethylaminooxoketene 1,3 migation of $\mathrm{NMe}_{2}$ Rotation TS (vacuum)

Geometry optimisation and frequency calculation: Program version = x86-Linux-G03RevB.05. Method and basis set = RB3LYP/6-31G(d). Calculated Energy = -399.9037713 (hartree), -1672.3975715766 (kJ mol ${ }^{-1}$ ). Higher level single-point calculation: Program = x86-Linux-G03RevB.05. Method and basis set = RB3LYP/6311++G(3df,2p). Calculated Energy = -400.0526807 (hartree), -1673.0203106874 (kJ mol $\left.{ }^{-1}\right)$. Structure definition (3D Coordinates: Atom, X coord/Å, Y coord/A, Z coord/Å): C, 0.8849012324, 1.3301913584, 0.0955119056; C, 1.5775726712, 0.1537121833, -0.108677182; O, 2.4729152273, -0.6023321992, 0.1469371513; C, -0.4924470542, 0.972369195, 0.0055262838; N, -0.3979240395, -0.52699493, 0.0410525498; O, -1.5359187657, 1.5837383354, 0.0575086336; H, 1.3365825488, 2.3122345393, 0.1527664026; C, -0.8682580162, -1.0929506099, 1.3121912641; C, -1.0291444241, -1.1608945505, 1.1238915577; H, -1.9511721588, -0.9559451359, 1.4350473998; H, -0.6343079391, -2.1615585892, 1.3373526389; H, -0.3527090304, -0.5989463282, 2.140253395; H, -2.1192530996, -1.0264618644, 1.1111217128; H, -0.628263735, -0.7159640001, -2.0386908423; H, -0.797126457, -2.2302086589, 1.119835598. Harmonic Frequencies (Number, Frequency $\left(\mathrm{cm}^{-1}\right)$, IR Intensity $\left(\mathrm{km} \mathrm{mol}^{-1}\right)$ ): 1, -122.4103 , 33.7168; 2, 82.7049, 3.9462; 3, 135.4143, 2.5865; 4, 205.9196, 3.1795; 5, 208.1522, 0.0361; 6, 248.437, 1.1197; 7, 292.2628, 3.4207; 8, 359.8989, 60.0901; 9, 390.2932, 21.1354; 10, 536.5727, 6.5205; 11, 613.0249, 7.7354; 12, 660.3853, 0.8713; 13, 704.6728, 48.3226; 14, 736.7781, 9.0307; 15, 773.7534, 11.6936; 16, 975.0115, 88.5859; 17, 1063.7175, 10.1648; 18, 1068.2072, 4.7767; 19, 1114.9309, 1.2829; 20, 1206.9411, 14.2261; 21, 1213.6155, 15.9854; 22, 1225.7313, 107.9820; 23, 1293.9976, 18.4184; 24, 1349.2429, 31.9950; 25, 1454.0311, 0.0267; 26, 1483.8388, 0.2828; 27, 1508.7996, 4.6640; 28, 1519.3418, 4.5712; 29, 1524.3586, 10.4875; 30, 1535.7758, 12.9736; 31, 1848.8322, 665.2169; 32, 2131.5387, 611.8270; 33, 3036.855, 24.9035; 34, 3041.7849, 50.5479; 35, 3115.5434, 4.4382; 36, 3116.3176, 25.3503; 37, 3147.0307, 10.0637; 38, 3148.6943, 19.1817; 39, 3258.359, 0.8951; 